\title{
Quantum Transport in Semiconductor Nanostructures
}

\author{
C W J Beenakker and H VAN Houten
}

Philips Research Laboratories

Eindhoven The Netherlands

$\begin{array}{lr}\text { I Introduction } & 1 \\ 1 \text { Preface } & 1 \\ 2 \text { Nanostructures in Si Inversion Layers } & 4 \\ 3 \text { Nanostructures in GaAs-AlGaAs Heterostructures } & 10 \\ 4 \text { Basic Properties } & 16 \\ \text { II Diffusive and Quasi-Ballıstıc Transport } & 26 \\ 5 \text { Classical Size Effects } & 26 \\ 6 \text { Weak Localization } & 34 \\ 7 \text { Conductance Fluctuations } & 49 \\ 8 \text { Aharonov-Bohm Effect } & 65 \\ 9 \text { Electron-Electron Interactions } & 71 \\ 10 \text { Quantum Size Effects } & 80 \\ 11 \text { Periodic Potential } & 86 \\ \text { Ballistic Transport } & 98 \\ 12 \text { Conduction as a Transmission Problem } & 98 \\ 13 \text { Quantum Point Contacts } & 109 \\ 14 \text { Coherent Electron Focusing } & 125 \\ 15 \text { Collımation } & 135 \\ 16 \text { Junction Scattering } & 146 \\ 17 \text { Tunneling } & 156 \\ \text { Adidbatic Transport } & 170 \\ 18 \text { Edge Channels and the Quantum Hall Effect } & 170 \\ 19 \text { Selective Population and Detection of Edge Channels } & 181 \\ 20 \text { Fractional Quantum Hall Effect } & 202 \\ 21 \text { Aharonov-Bohm Effect in Strong Magnetıc Fields } & 215 \\ 22 \text { Magnetically Induced Band Structure } & 223\end{array}$

\section{Introduction}

\section{PRerace}

In recent years semiconductor nanostructures have become the model systems of choice for investigations of electrical conduction on short length scales This development was made possible by the avallability of semiconductıng materials of unprecedented purity and crystalline perfection Such materials can be structured to contain a thin layer of highly mobile electrons Motion perpendicular to the layer is quantized, so that the electrons are 
constrained to move in a plane As a model system, this two-dimensional electron gas ( $2 D E G$ ) combines a number of desirable properties, not shared by thin metal films It has a low electron density, which may be readily varied by means of an electric field (because of the large screening length) The low density implies a large Fermı wavelength (typically $40 \mathrm{~nm}$ ), comparable to the dimensions of the smallest structures (nanostructures) that can be fabricated today The electron mean free path can be quite large (exceeding $10 \mu \mathrm{m}$ ) Finally, the reduced dimensionality of the motion and the circular Fermi surface form simplifying factors

Quantum transport is conveniently studied in a 2DEG because of the combination of a large Fermi wavelength and large mean free path The quantum mechanical phase coherence characteristic of a microscopic object can be maintained at low temperatures (below $1 \mathrm{~K}$ ) over distances of several microns, which one would otherwise have classified as macroscopic The physics of these systems has been referred to as mesoscopic, ${ }^{1}$ a word borrowed from statistical mechanics ${ }^{2}$ Elastic impurity scatterıng does not destroy phase coherence, which is why the effects of quantum interference can modify the conductivity of a disordered conductor This is the regime of diffusive transport, characteristic for disordered metals Quantum interference becomes more important as the dimensionality of the conductor is reduced Quası-one dimensionality can readily be achieved in a 2DEG by lateral confinement

Semiconductor nanostructures are unique in offering the possibility of studying quantum transport in an artificial potential landscape This is the regime of ballistic transport, in which scattering with impurities can be neglected The transport properties can then be tallored by varying the geometry of the conductor, in much the same way as one would tallor the transmission properties of a wavegurde The physics of this transport regime could be called electron optics in the solid state ${ }^{3}$ The formal relation between conduction and transmission, known as the Landauer formula, ${ }^{145}$ has demonstrated its real power in this context For example, the quantization of the conductance of a quantum point $\operatorname{contact}^{67}$ (a short and narrow

${ }^{1} Y$ Imry, in "Directions in Condensed Matter Physıcs," Vol 1 (G Grınstem and G Mazenko, eds ) World Scientific, Singapore, 1986

${ }^{2} \mathrm{~N} G$ van Kampen, "Stochastıc Processes in Physics and Chemistry" North-Holland, Amsterdam, 1981

${ }^{3} \mathrm{H}$ van Houten and C W J Beenakker, in "Analogies in Optics and Microelectronıcs" (W van Haeringen and D Lenstra, eds) Kluwer Academic, Dordrecht 1990

${ }^{4} \mathrm{R}$ Landauer, IBM $J$ Res Dev 1, 223 (1957), 32, 306 (1988)

${ }^{5} \mathrm{M}$ Buttiker, Phys Rev Lett 57, 1761 (1986)

${ }^{6} \mathrm{~B}$ J van Wees, $\mathrm{H}$ van Houten, C W J Beenakker, J G Willamson, L P Kouwenhoven, D van der Marel, and C T Foxon, Phys Rev Lett 60848 (1988)

${ }^{7} \mathrm{D}$ A Wharam, T J Thornton, R Newbury, M Pepper, H Ahmed, J E F Frost, D G Hasko, D C Peacock, D A Ritchie, and G A C Jones, J Phys C 21, L209 (1988) 
constriction in the 2DEG) can be understood using the Landauer formula as resulting from the discreteness of the number of propagating modes in a waveguide.

Two-dimensional systems in a perpendicular magnetic field have the remarkable property of a quantized Hall resistance, ${ }^{8}$ which results from the quantization of the energy in a series of Landau levels. The magnetic length $(\hbar / e B)^{1 / 2}(\approx 10 \mathrm{~nm}$ at $B=5 \mathrm{~T})$ assumes the role of the wavelength in the quantum Hall effect. The potential landscape in a $2 \mathrm{DEG}$ can be adjusted to be smooth on the scale of the magnetic length, so that inter-Landau level scattering is suppressed. One then enters the regime of adlabatic transport. In this regime truly macroscopic behavior may not be found even in samples as large as $0.25 \mathrm{~mm}$.

In this review we present a self-contained account of these three novel transport regimes in semiconductor nanostructures. The experimental and theoretical developments in this field have developed hand in hand, a fruitful balance that we have tried to maintain here as well. We have opted for the simplest possible theoretical explanations, avoiding the powerful-but more formal-Green's function techniques. If in some instances this choice has not enabled us to do full justice to a subject, then we hope that this disadvantage is compensated by a gain in accessibility. Lack of space and time has caused us to limit the scope of this review to metallic transport in the plane of a 2DEG at small currents and voltages. Transport in the regime of strong localization is excluded, as well as that in the regime of a nonlinear currentvoltage dependence. Overviews of these, and other, topics not covered here may be found in Refs. 9-11, as well as in recent conference proceedings. ${ }^{12-17}$

We have attempted to give a comprehensive list of references to theoretical

${ }^{8} \mathrm{~K}$ von Klitzing, G Dorda, and M Pepper, Phys Rev Lett 45, 494 (1980)

${ }^{9} \mathrm{M}$ A Reed, ed, "Nanostructured Systems" Academic Press, New York, to be published

${ }^{10} \mathrm{P}$ A Lee, R A Webb and B L Al'tshuler, eds, "Mesoscopic Phenomena in Solids" Elsevier, Amsterdam, to be published

${ }^{11} \mathrm{~B}$ L Al'tshuler, R A Webb, and R B Laibowitz, eds, IBM $J$ Res Dev 32, 304-437, 439-579 (1988)

12 "Proceedings of the International Conference on Electronic Properties of Two-Dimensional Systems," IV-VIII, Suif Scl 113 (1982), 142 (1984), 170 (1986), 196 (1988), 229 (1990)

${ }^{13} \mathrm{M}$ J Kelly and C Weisbuch, eds, "The Physics and Fabrication of Microstructures and Microdevices" Proc Winter School Les Houches, 1986, Springer, Berlin, 1986

${ }^{14} \mathrm{H}$ Heinrich, G Bauer, and F Kuchar, eds, "Physics and Technology of Submicron Structures" Sprınger, Berlın, 1988

${ }^{15} \mathrm{M}$ Reed and W P Kirk, eds, "Nanostructure Physics and Fabrication " Academic Press, New York, 1989

${ }^{16} \mathrm{~S}$ P Beaumont and C M Sotomayor-Torres, eds, "Science and Engıneering of 1- and 0Dimensional Semiconductors" Plenum, London, 1990

${ }^{17} \mathrm{~J}$ M Chamberlain, L Eaves, and J C Portal, eds, "Electronıc Properties of Multilayers and Low-Dimensional Semiconductor Structures" Plenum, London, to be published 
and experimental work on the subjects of this review We apologize to those whose contributions we have overlooked Certain experiments are discussed in some detall In selecting these experiments, our aum has been to choose those that illustrate a particular phenomenon in the clearest fashion, not to establısh priorities We thank the authors and publishers for their kind permission to reproduce figures from the original publications Much of the work reviewed here was a joint effort with colleagues at the Delft University of Technology and at the Philips Research Laboratories, and we are grateful for the stimulating collaboration

The study of quantum transport in semiconductor nanostructures is motivated by more than scientific interest The fabrication of nanostructures relies on sophisticated crystal growth and lithographic techniques that exist because of the industrial effort toward the miniaturization of transistors Conventional transistors operate in the regime of classical diffusive transport, which breaks down on short length scales The discovery of novel transport regimes in semiconductor nanostructures provides options for the development of innovative future devices At this point, most of the proposals in the literature for a quantum interference device have been presented primarily as interesting possibilities, and they have not yet been critically analyzed A quantitative comparison with conventional transistors will be needed, taking circuit design and technological considerations into account ${ }^{18}$ Some proposals are very ambitious, in that they do not only consider a different principle of operation for a single transistor, but envision entire computer architectures in which arrays of quantum devices operate phase coherently ${ }^{19}$

We hope that the present review will convey some of the excitement that the workers in this rewarding field of research have experienced in its exploration May the description of the variety of phenomena known at present, and of the simplest way in which they can be understood, form an inspiration for future investigations

\section{NaNOSTRUCTURES IN S1 INVERSION LAyERS}

Electronic properties of the two-dimensional electron gas in S1 MOSFETs (metal-oxide-semiconductor field-effect transistors) have been reviewed by Ando, Fowler, and Stern, ${ }^{20}$ while general technological and device aspects are covered in detail in the books by Sze ${ }^{21}$ and by Nicollian and Brew ${ }^{22}$ In this section we only summarize those properties that are needed in the

\footnotetext{
${ }^{18} \mathrm{R}$ Landauer, Phys Today 42, 119 (1989)

${ }^{19} \mathrm{R}$ T Bate, $S C l$ Am 25878 (1988)

${ }^{20} \mathrm{~T}$ Ando, A B Fowler, and F Stern, Rev Mod Phys 54, 437 (1982)

${ }^{21}$ S M Sze, "Physics of Semiconductor Devices" Wiley, New York, 1981

${ }^{22}$ E H Nicollian and J R Brew, "Metal Oxide Semiconductor Technology" Wiley, New York, 1982
} 


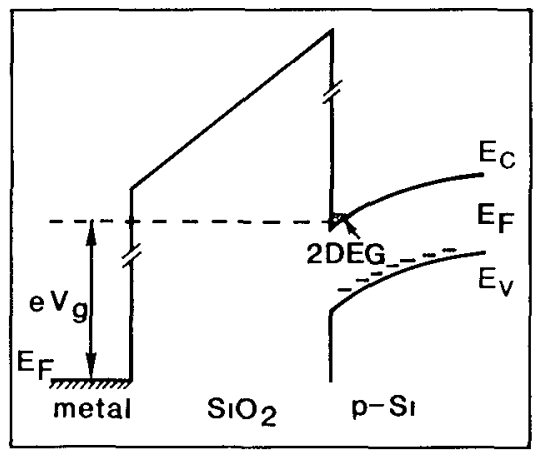

FIG 1 Band-bending diagram (showing conduction band $E_{\mathrm{C}}$, valence band $E_{\mathrm{V}}$, and Ferm 1 level $E_{\mathrm{F}}$ ) of a metal-oxide-semiconductor (MOS) structure A 2DEG is formed at the interface between the oxide and the p-type silicon substrate, as a consequence of the positive voltage $V_{g}$ on the metal gate electrode

following A typical device consists of a p-type $\mathrm{S} i$ substrate, covered by a $\mathrm{SiO}_{2}$ layer that serves as an insulator between the (100) Si surface and a metallic gate electrode By application of a sufficiently strong positive voltage $V_{\mathrm{g}}$ on the gate, a 2DEG is induced electrostatically in the $p$-type $S_{1}$ under the gate The band bending leading to the formation of this inversion layer is schematically indicated in Fig 1 The areal electron concentration (or sheet density) $n_{\mathrm{s}}$ follows from $e n_{\mathrm{s}}=C_{\mathrm{ox}}\left(V_{\mathrm{g}}-V_{\mathrm{t}}\right)$, where $V_{\mathrm{t}}$ is the threshold voltage beyond which the inversion layer is created, and $C_{\text {ox }}$ is the capacitance per unit area of the gate electrode with respect to the electron gas Approximately, one has $C_{\mathrm{ox}}=\varepsilon_{\mathrm{ox}} / d_{\mathrm{ox}}$ (with $\varepsilon_{\mathrm{ox}}=39 \varepsilon_{0}$ the dielectric constant of the $\mathrm{SiO}_{2}$ layer), ${ }^{21}$ so

$$
n_{\mathrm{s}}=\frac{\varepsilon_{\mathrm{ox}}}{e d_{\mathrm{ox}}}\left(V_{\mathrm{g}}-V_{\mathrm{t}}\right)
$$

The linear dependence of the sheet density on the applied gate voltage is one of the most useful properties of Si inversion layers

The electric field across the oxide layer resulting from the applied gate voltage can be quite strong Typically, $V_{\mathrm{g}}-V_{\mathrm{t}}=5 \mathrm{~V}$ and $d_{\mathrm{ox}}=50 \mathrm{~nm}$, so the field strength is of order $1 \mathrm{MV} / \mathrm{cm}$, at best a factor of 10 lower than typical fields for the dielectric breakdown of $\mathrm{S}_{1} \mathrm{O}_{2}$ It is possible to change the electric field at the interface, without altering $n_{\mathrm{s}}$, by applying an additional voltage across the $p-n$ junction that isolates the inversion layer from the $p$-type substrate (such a voltage is referred to as a substrate bias) At the $\mathrm{S} 1-\mathrm{S}_{1} \mathrm{O}_{2}$ interface the electric field is continuous, but there is an electrostatic potential step of about $3 \mathrm{eV}$ An approximately triangular potential well is thus formed 
at the interface (see Fig. 1). The actual shape of the potential deviates somewhat from the triangular one due to the electronic charge in the inversion layer, and has to be calculated self-consistently. ${ }^{20}$ Due to the confinement in one direction in this potential well, the three-dimensional conduction band splits into a series of two-dimensional subbands. Under typical conditions (for a sheet electron density $n_{\mathrm{s}}=10^{11}-10^{12} \mathrm{~cm}^{-2}$ ) only a single two-dimensional subband is occupied. Bulk Si has an indirect band gap, with six equivalent conduction band valleys in the $\langle 100\rangle$ direction in reciprocal space. In inversion layers on the (100) Si surface, the degeneracy between these valleys is partially lifted. A twofold valley degeneracy remains. In the following, we treat these two valleys as completely independent, ignoring complications due to intervalley scattering. For each valley, the (one-dimensional) Fermi surface is simply a circle, corresponding to free motion in a plane with effective electron mass ${ }^{20} m=0.19 m_{\mathrm{e}}$. For easy reference, this and other relevant numbers are listed in Table I.

The electronic properties of the $\mathrm{Si}$ inversion layer can be studied by capacitive or spectroscopic techniques (which are outside the scope of this review), as well as by transport measurements in the plane of the 2DEG. To determine the intrinsic transport properties of the 2DEG (e.g., the electron mobility), one defines a wide channel by fabricating a gate electrode with the appropriate shape. Ohmic contacts to the channel are then made by ion implantation, followed by a lateral diffusion and annealing process. The two current-carrying contacts are referred to as the source and the drain. One of these also serves as zero reference for the gate voltage. Additional side contacts to the channel are often fabricated as well (for example, in the Hall bar geometry), to serve as voltage probes for measurements of the longitudinal and Hall resistance. Insulation is automatically provided by the $p-n$ junctions surrounding the inversion layer. (Moreover, at the low temperatures of interest here, the substrate conduction vanishes anyway due to carrier freeze-out.) The electron mobility $\mu_{\mathrm{e}}$ is an important figure of merit for the quality of the device. At low temperatures the mobility in a given sample varies nonmonotonically ${ }^{20}$ with increasing electron density $n_{\mathrm{s}}$ (or increasing gate voltage), due to the opposite effects of enhanced screening (which reduces ionized impurity scattering) and enhanced confinement (which leads to an increase in surface roughness scattering at the $\mathrm{Si}-\mathrm{SiO}_{2}$ interface). The maximum low-temperature mobility of electrons in high-quality samples is around $10^{4} \mathrm{~cm}^{2} / \mathrm{V} \cdot \mathrm{s}$. This review deals with the modifications of the transport properties of the 2DEG in narrow geometries. Several lateral confinement schemes have been tried in order to achieve narrow inversion layer channels (see Fig. 2). Many more have been proposed, but here we discuss only those realized experimentally.

Technically simplest, because it does not require electron beam lithography, is an approach first used by Fowler et al., following a suggestion by 
Table I Electronic Properties of the 2DEG in GaAs-AlGaAs Heterostructures and Si INVERSION LAYERS

\begin{tabular}{|c|c|c|c|c|}
\hline & & $\operatorname{GaAs}(100)$ & $S_{1}(100)$ & UNITS \\
\hline Effectıve Mass & $m$ & 0067 & 019 & $m_{\mathrm{c}}=91 \times 10^{-28} \mathrm{~g}$ \\
\hline Spin Degeneracy & $g_{\mathrm{s}}$ & 2 & 2 & \\
\hline Valley Degeneracy & $g_{\mathrm{v}}$ & 1 & 2 & \\
\hline Dielectric Constant & $\varepsilon$ & 131 & 119 & $\begin{aligned} \varepsilon_{0}= & 89 \\
& \times 10^{-12} \mathrm{Fm}^{-1}\end{aligned}$ \\
\hline $\begin{array}{l}\text { Density of States } \\
\text { Electronic Sheet }\end{array}$ & $\rho(E)=g_{\varsigma} g_{v}\left(m / 2 \pi \hbar^{2}\right)$ & 028 & 159 & $10^{11} \mathrm{~cm}^{-2} \mathrm{meV}^{-1}$ \\
\hline Density & $n_{\mathrm{s}}$ & 4 & $1-10$ & $10^{11} \mathrm{~cm}^{-2}$ \\
\hline Fermı Wave Vector & $k_{\mathrm{F}}=\left(4 \pi n_{\mathrm{s}} / g_{\mathrm{s}} g_{\mathrm{v}}\right)^{1 / 2}$ & 158 & $056-177$ & $10^{6} \mathrm{~cm}^{-1}$ \\
\hline Fermı Velocity & $v_{\mathrm{F}}=\hbar k_{\mathrm{F}} / m$ & 27 & $034-11$ & $10^{7} \mathrm{~cm} / \mathrm{s}$ \\
\hline Fermı Energy & $E_{\mathrm{F}}=\left(\hbar k_{\mathrm{F}}\right)^{2} / 2 m$ & 14 & $063-63$ & $\mathrm{meV}$ \\
\hline Electron Mobility ${ }^{\mathrm{a}}$ & $\mu_{\mathrm{c}}$ & $10^{4}-10^{6}$ & $10^{4}$ & $\mathrm{~cm}^{2} / \mathrm{Vs}$ \\
\hline Scatterıng Time & $\tau=m \mu_{\mathrm{c}} / e$ & $038-38$ & 11 & $\mathrm{ps}$ \\
\hline Diffusion Constant & $D=v_{\mathrm{F}}^{2} \tau / 2$ & $140-14000$ & $64-64$ & $\mathrm{~cm}^{2} / \mathrm{s}$ \\
\hline Resistıvity & $\rho=\left(n_{s} e \mu_{\mathrm{c}}\right)^{-1}$ & $16-0016$ & $63-063$ & $\mathrm{k} \Omega$ \\
\hline Fermı Wavelength & $\lambda_{\Gamma}=2 \pi / k_{\Gamma}$ & 40 & $112-35$ & $\mathrm{~nm}$ \\
\hline Mean Free Path & $l=v_{\mathrm{F}} \tau$ & $10^{2}-10^{4}$ & $37-118$ & $\mathrm{~nm}$ \\
\hline \multicolumn{5}{|l|}{ Phase Coherence } \\
\hline Length ${ }^{b}$ & $l_{\phi}=\left(D \tau_{\phi}\right)^{1 / 2}$ & $200-$ & $40-400$ & $\mathrm{~nm}(T / \mathrm{K})^{-1 / 2}$ \\
\hline Thermal Length & $l_{\mathrm{T}}=\left(h D / k_{\mathrm{B}} T\right)^{1 / 2}$ & $330-3300$ & $70-220$ & $\mathrm{~nm}(T / \mathrm{K})^{-1 / 2}$ \\
\hline Cyclotron Radius & $l_{c y c l}=\hbar k_{\mathrm{F}} / e B$ & 100 & $37-116$ & $\mathrm{~nm}(B / \mathrm{T})^{-1}$ \\
\hline \multirow[t]{4}{*}{ Magnetic Length } & $l_{\mathrm{m}}=(h / e B)^{1 / 2}$ & 26 & 26 & $\operatorname{nm}(B / \mathrm{T})^{-1 / 2}$ \\
\hline & $k_{\mathrm{F}} l$ & $158-1580$ & $21-21$ & \\
\hline & $\omega_{\mathrm{c}} \tau$ & $1-100$ & 1 & $(B / \mathrm{T})$ \\
\hline & $E_{\mathrm{F}} / h \omega_{\mathrm{c}}$ & 79 & $1-10$ & $(B / T)^{-1}$ \\
\hline
\end{tabular}

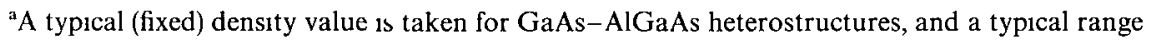
of values in the metallic conduction regime for S1 MOSFETs For the mobility, a range of representative values is listed for GaAs-AlGaAs heterostructures, and a typical "good" value for Si MOSFETs The variation in the other quantities reflects that in $n_{\mathrm{s}}$ and $\mu_{\mathrm{c}}$ ${ }^{b}$ Rough estimate of the phase coherence length, based on weak localization experiments in laterally confined heterostructures ${ }^{23-27}$ and Si MOSFETs ${ }^{28}{ }^{29}$ The stated $T^{-1 / 2}$ temperature dependence should be regarded as an indication only, since a simple power law dependence is not always found (see, for example, Refs 30 and 25) For high-mobility GaAs-AlGaAs heterostructures the phase coherence length is not known, but is presumably ${ }^{31}$ comparable to the (elastic) mean free path $l$

${ }^{23}$ B J F Lin, M A Paalanen, A C Gossard, and D C Tsul, Phys Rev B 29, 927 (1984)

${ }^{24} \mathrm{H} \mathrm{Z}$ Zheng, H P We1, D C Tsul, and G Weimann Phys Rev B 34, 5635 (1986)

${ }^{25} \mathrm{~K}$ K Cho,, D C Tsui, and K Alav1, Phys Rev B 36, 7751 (1987), Appl Phys Lett 50, 110 (1987)

${ }^{26} \mathrm{H}$ van Houten, C W J Beenakker, B J van Wees, and J E Moou, Surf Scl 196, 144 (1988)

${ }^{27} \mathrm{H}$ van Houten, C W J Beendkker, M E I Broekdart, M G J Heıman, B J van Wees, J E Mooly, and J P Andre, Acta Electionica, 28, 27 (1988)

${ }^{28} \mathrm{D} J$ Bishop, R C Dynes, and D C Tsui, Phys Rev B 26, 773 (1982)

${ }^{29}$ W J Skocpol, L D Jackel, E L Hu, R E Howard, and L A Fetter, Phys Rev Lett 49, 951 (1982)

${ }^{30} \mathrm{~K}$ K Cho1, Phys Rev B 28, 5774 (1983)

${ }^{31} \mathrm{H}$ van Houten, B J van Wees, and C W J Beenakker, in Ref 14 
a

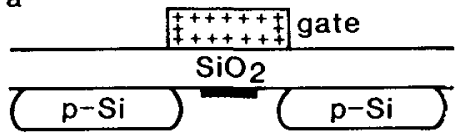

$\mathrm{n}-\mathrm{S} \mathbf{I}$

C

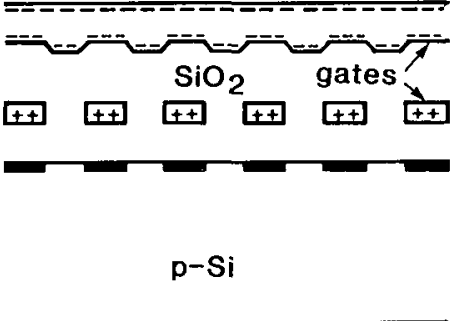

b

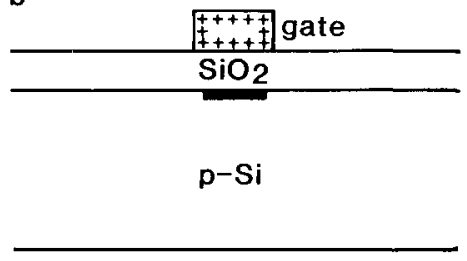

d

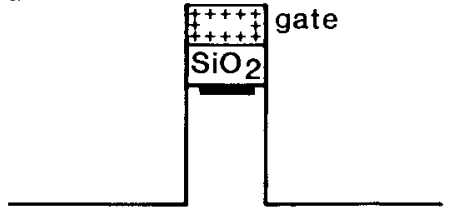

$\mathrm{p}-\mathrm{Si}$

FIG 2 Schematic cross-sectional views of the lateral pinch-off technique used to define a narrow electron accumulation layer (a), and of three different methods to define a narrow inversion layer in S1 MOSFETs $(b-d)$ Positive $(+)$ and negative $(-)$ charges on the gate electrodes are indicated The location of the 2DEG is shown in black

Pepper ${ }^{32-34}$ (Fig. 2a). By adjusting the negatıve voltage over $p$ - $n$ junctions on either side of a relatively wide gate, they were able to vary the electron channel width as well as its electron density. This technique has been used to define narrow accumulation layers on $n$-type $\mathrm{Si}$ substrates, rather than inversion layers. Specifically, it has been used for the exploration of quantum transport in the strongly localized regime ${ }^{32,35-37}$ (which is not discussed in this review). Perhaps the technique is particularly suited to this highly resistive regime, since a tail of the diffusion profile inevitably extends into the channel, providing additional scattering centers. ${ }^{34}$ Some studies in the weak localization regime have also been reported. ${ }^{33}$

The conceptually simplest approach (Fig. $2 b$ ) to define a narrow channel is to scale down the width of the gate by means of electron beam lithography ${ }^{38}$

\footnotetext{
${ }^{32}$ A B Fowler, A Hartstem, and R A Webb, Phys Rev Lett 48, 196 (1982)

${ }^{33} \mathrm{M}$ Pepper and M J Uren, $J$ Phys C 15, L617 (1982)

${ }^{34} \mathrm{C}$ C Dean and M Pepper, $J$ Phys C 15, L1287 (1982)

${ }^{35}$ A B Fowler, J J Wainer, and R A Webb, IBM J Res Dev 32, 372 (1988)

${ }^{36}$ S B Kaplan and A C Warren, Phys Rev B 34, 1346 (1986)

${ }^{37}$ S B Kaplan and A Hartstem, IBM J Res Dev 32, 347 (1988), Phys Rev Lett 56, 2403 (1986)

${ }^{38}$ R G Wheeler, K K Chol, A Goel, R Wisnieff, and D E Prober, Phys Rev Lett 49, 1674 (1982)
} 
or other advanced techniques ${ }^{39-41}$ A difficulty for the characterization of the device is that fringing fields beyond the gate induce a considerable uncertainty in the channel width, as well as its density Such a problem is shared to some degree by all approaches, however, and this technique has been quite successful (as we will discuss in Section II) For a theoretical study of the electrostatic confining potential induced by the narrow gate, we refer to the work by Laux and Stern ${ }^{42}$ This is a complicated problem, which requires a self-consistent solution of the Poisson and Schrodinger equations, and must be solved numerically

The narrow gate technique has been modified by Warren et al ${ }^{4344}$ (F1g 2c), who covered a multiple narrow-gate structure with a second dielectric followed by a second gate covering the entire device (This structure was specifically intended to study one-dimensional superlattice effects, which is why multiple narrow gates were used ) By separately varying the voltages on the two gates, one achieves an increased control over channel width and density The electrostatics of this particular structure has been studied in Ref 43 in a semiclassical approximation

Skocpol et $\mathrm{al}^{2945}$ have combined a narrow gate with a deep self-aligned mesa structure (Fig 2d), fabricated using dry-etching techniques One advantage of their method is that at least an upper bound on the channel width is known unequivocally A disadvantage is that the deep etch exposes the sidewalls of the electron gas, so that it is likely that some mobility reduction occurs due to sidewall scattering In addition, the deep etch may damage the 2DEG itself This approach has been used successfully in the exploration of nonlocal quantum transport in multiprobe channels, which in addition to being narrow have a very small separation of the voltage probes ${ }^{45}{ }^{46}$ In another investigation these narrow channels have been used as instruments sensitive to the charging and discharging of a single electron trap, allowing a detailed study of the statistics of trap kinetics ${ }^{46-48}$

\footnotetext{
${ }^{39}$ R F Kwasnick, M A Kastner, J Melngalls, and P A Lee, Phys Rev Lett 52, 224 (1984)

${ }^{40}$ J C Licinı, D J Bıshop, M A Kastner, and J Melngallı, Phys Rev Lett 55, 2987 (1985)

${ }^{41}$ P H Woerlee, G A M Hurkx, W J M J Josquin, and J F C M Verhoeven, Appl Phys Lett 47, 700 (1985), see also H van Houten and P H Woerlee, "Proc ICPS 18, p 1515 (O Engstrom, ed) World Scientific, Singapore, 1987

${ }^{42}$ S E Laux and F Stern, Appl Phys Lett 49, 91 (1986)

${ }^{43}$ A C Warren, D A Antoniadis, and H I Smith, Phys Rev Lett 56, 1858 (1986)

${ }^{44}$ A C Warren, D A Antoniadis, and H I Smith, IEEE Electron Device Lett, EDL-7, 413 (1986)

${ }^{45}$ W J Skocpol, P M Mankiewich, R E Howard, L D Jackel, D M Tennant, and A D Stone, Phys Rev Lett 56, 2865 (1986)

${ }^{46}$ W J Skocpol, Physica Scripta T19, 95 (1987)

${ }^{47} \mathrm{~K}$ S Ralls, W J Skocpol, L D Jackel, R E Howard, L A Fetter, R W Epworth, and D M Tennant, Phys Rev Lett 52, 228 (1984)

${ }^{48} \mathrm{R}$ E Howard, W J Skocpol, L D Jackel, P M Mankiewıch, L A Fetter, D M Tennant, R Epworth, and K S Ralls, IEEE Trans ED-32, 1669 (1985)
} 


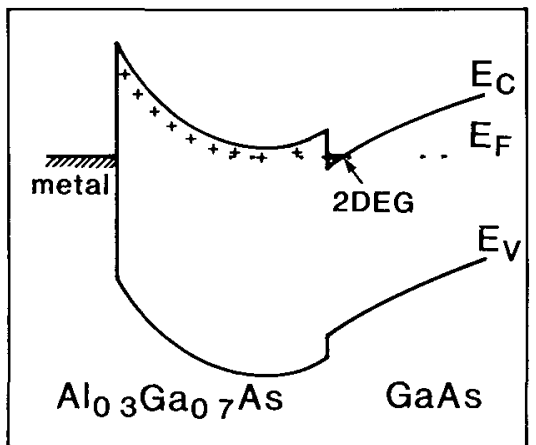

FIG 3 Band-bending diagram of a modulation doped $\mathrm{GaAs}_{-} \mathrm{Al}_{x} \mathrm{Ga}_{1-x}$ As heterostructure A 2DEG is formed in the undoped GaAs at the interface with the p-type doped AlGaAs Note the Schottky barrier between the semiconductor and a metal electrode

\section{Nanostructures in GaAs-AlGaAs Heterostructures}

In a modulation-doped ${ }^{49} \mathrm{GaAs}-\mathrm{AlGaAs}$ heterostructure, the $2 \mathrm{DEG}$ is present at the interface between $\mathrm{GaAs}$ and $\mathrm{Al}_{x} \mathrm{Ga}_{1-x} \mathrm{As}$ layers (for a recent review, see Ref 50) Typically, the Al mole fraction $x=03$ As shown in the band-bending diagram of Fig 3, the electrons are confined to the GaAsAlGaAs interface by a potential well, formed by the repulsıve barner due to the conduction band offset of about $03 \mathrm{~V}$ between the two semiconductors, and by the attractive electrostatic potential due to the positively charged ionized donors in the $n$-doped AlGaAs layer To reduce scattering from these donors, the doped layer is separated from the interface by an undoped AlGaAs spacer layer Two-dimensional subbands are formed as a result of confinement perpendicular to the interface and free motion along the interface An important advantage over a MOSFET is that the present interface does not interrupt the crystalline periodicity This is possible because GaAs and $\mathrm{AlGaAs}$ have almost the same lattice spacing Because of the absence of boundary scattering at the interface, the electron mobility can be higher by many orders of magnitude (see Table I) The mobility is also high because of the low effective mass $m=0067 m_{\mathrm{c}}$ in GaAs (for a review of GaAs material properties, see Ref 51) As in a Si inversion layer, only a single twodimensional subband (associated with the lowest discrete confinement level in the well) is usually populated Since GaAs has a direct band gap, with a

\footnotetext{
${ }^{49} \mathrm{H}$ L Stormer, R Dingle, A C Gossard, and W Wiegman, "Proc 14th ICPS," p 6(B L H Wilson, ed ) Institute of Physics, London, 1978, R Dingle, H L Stormer, A C Gossard, and W Wiegman, Appl Phys Lett 7, 665 (1978)

${ }^{50}$ J J Harris, J A Pals and R Woltjer, Rep Prog Phys 52, 1217 (1989)

${ }^{51}$ S Adach, $J$ Appl Phys 58, R1 (1985)
} 
b

a

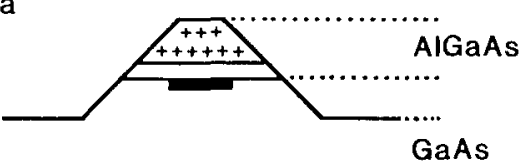

GaAs

C

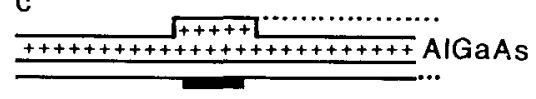

GaAs

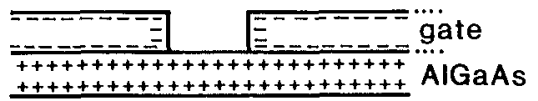

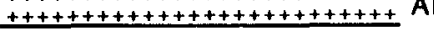
...

GaAs

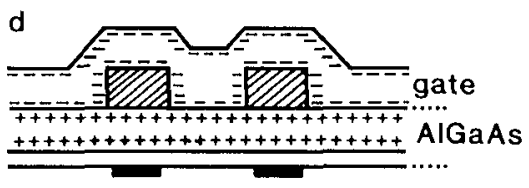

GaAs

FIG 4 Schematic cross-sectional views of four different ways to define narrow 2DEG channels in a GaAs-AlGaAs heterostructure Positive ionized donors and negative charges on a Schottky gate electrode are indicated The hatched squares in $\mathrm{d}$ represent unremoved resist used as a gate dielectric

single conduction band minimum, complications due to intervalley scattering (as in $\mathrm{Si}$ ) are absent. The one-dimensional Fermi surface is a circle, for the commonly used (100) substrate orientation.

Since the 2DEG is present "naturally" due to the modulation doping (i.e., even in the absence of a gate), the creation of a narrow channel now requires the selective depletion of the electron gas in spatially separated regions. In principle, one could imagine using a combination of an undoped heterostructure and a narrow gate (similarly to a MOSFET), but in practice this does not work very well due to the lack of a natural oxide to serve as an insulator on top of the AlGaAs. The Schottky barrier between a metal and (Al)GaAs (see Fig. 3) is too low (only $0.9 \mathrm{~V}$ ) to sustain a large positive voltage on the gate. For depletion-type devices, where a negative voltage is applied on the gate, the Schottky barrier is quite sufficient as a gate insulator (see, e.g., Ref. 52).

The simplest lateral confinement technique is illustrated in Fig. 4a. The

${ }^{52} \mathrm{D}$ Delagebeaudeuf and N T Linh, IEEE Trans ED-28, 790 (1981) 
appropriate device geometry (such as a Hall bar) is realızed by defining a deep mesa, by means of wet chemical etching Wide Hall bars are usually fabricated in this way This approach has also been used to fabricate the first micron-scale devices, such as the constrictions used in the study of the breakdown of the quantum Hall effect by Kurtley et al ${ }^{53}$ and Bliek et al,$^{54}$ and the narrow channels used in the first study of quasi-one-dimensional quantum transport in heterostructures by Cho1 et al ${ }^{55}$ The deep-mesa confinement technique using wet ${ }^{25}{ }^{56}$ or dry ${ }^{57}$ etching is still of use for some experimental studies, but it is generally felt to be unreliable for channels less than $1 \mu \mathrm{m}$ wide (in particular because of the exposed sidewalls of the structure)

The first working alternative confinement scheme was developed by Thornton et $a l^{58}$ and Zheng et al, ${ }^{24}$ who introduced the split-gate lateral confinement technique ( $\left.F_{1 g} 4 b\right)$ On application of a negatıve voltage to a split Schottky gate, wide 2DEG regions under the gate are depleted, leaving a narrow channel undepleted The most appealing feature of this confinement scheme is that the channel width and electron density can be varied continuously (but not independently) by increasing the negative gate voltage beyond the depletion threshold in the wide regions (typically about $-06 \mathrm{~V}$ ) The split-gate technique has become very popular, especially after it was used to fabricate the short and narrow constrictions known as quantum point contacts $^{6599}$ (see Section III) The electrostatic confinement problem for the split-gate geometry has been studied numerically in Refs 60 and 61 A simple analytical treatment is given in Ref 62 A modification of the split-gate technique is the grating-gate technique, which may be used to define a 2DEG with a periodic density modulation ${ }^{62}$

The second widely used approach is the shallow-mesa depletion technique (F1g 4c), introduced in Ref 63 This technique relies on the fact that a $2 \mathrm{DEG}$

${ }^{53}$ J P Kirtley, Z Schlesinger, T N Theis, F P Milliken, S L Wright, and L F Palmateer, Phys Rev B 34, 5414 (1986)

${ }^{54}$ L Bliek, E Braun, G Hein, V Kose, J Niemeyer, G Wermann, and W Schlapp, Semicond Scl Technol 1, 110 (1986)

${ }^{55} \mathrm{~K}$ K Chol, D C Tsui, and S C Palmateer, Phys Rev B 33, 8216 (1986)

${ }^{56}$ A D C Grassie, K M Hutchıngs, M Lakrım1, C T Foxon, and J J Harris, Phys Rev B 36, 4551 (1987)

${ }^{57}$ T Demel, D Hettmann, P Grambow, and K Ploog, Appl Phys Lett 53, 2176 (1988)

${ }^{58} \mathrm{~T}$ J Thornton, M Pepper, H Ahmed, D Andrews, and G J Davies, Phys Rev Lett 56, 1198 (1986)

${ }^{59} \mathrm{H}$ van Houten, B J van Wees, J E Mooij, C W J Beenakker, J G Williamson, and C $\mathrm{T}$ Foxon, Europhys Lett 5, 721 (1988)

${ }^{60}$ S E Laux, D J Frank, and F Stern, Surf Scl 196, 101 (1988)

${ }^{61}$ A Kumar, S E Laux, and F Stern, Appl Phys Lett 54, 1270 (1989)

${ }^{62} \mathrm{~K}$ Ismal, W Chu D A Antoniadis, dnd H I Smith, Appl Phys Lett 52, 1071 (1988)

${ }^{63} \mathrm{H}$ van Houten, B J van Wees, M G J Heljman, and J P Andre, Appl Phys Lett 49, 1781 (1986) 
can be depleted by removal of only a thin layer of the AlGaAs, the required thickness being a sensitive function of the parameters of the heterostructure material, and of detals of the lithographic process (which usually involves electron beam lithography followed by dry etching) The shallow-mesa etch technique has been perfected by two groups, ${ }^{64-66}$ for the fabrication of multıprobe electron waveguides and rings ${ }^{67-70}$ Submicron trenches ${ }^{71}$ are still another way to define the channel For simple analytical estimates of lateral depletion widths in the shallow-mesa geometry, see Ref 72

A clever variant of the split-gate technique was introduced by Ford et $a l^{73}{ }^{74} \mathrm{~A}$ patterned layer of electron beam resist (an organic insulator) is used as a gate dielectric, in such a way that the separation between the gate and the 2DEG is largest in those regions where a narrow conductıng channel has to remain after application of a negatıve gate voltage As illustrated by the crosssectional view in Fig 4d, in this way one can define a ring structure, for example, for use in an Aharonov-Bohm experiment A similar approach was developed by Smith et al ${ }^{75}$ Instead of an organic resist they use a shallowmesa pattern in the heterostructure as a gate dielectric of varıable thickness Initially, the latter technique was used for capacitive studies of one- and zerodimensional confinement ${ }^{75} 76$ More recently it was adopted for transport measurements as well ${ }^{77}$ Still another variation of this approach was

${ }^{64} \mathrm{R}$ E Behringer P M Mankiewich and R E Howard $J$ Vac Scl Technol B5, 326 (1987)

${ }^{65}$ A Scherer M L Roukes, H G Craighead, R M Ruthen, E D Beebe, and J P Harbison, Appl Phys Lett 51, 2133 (1987)

${ }^{66} \mathrm{~A}$ Scherer and M L Roukes, Appl Phys Lett 55, 377 (1989)

${ }^{67} \mathrm{M}$ L Roukes, A Scherer, S J Allen, Jr, H G Craighead, R M Ruthen, E D Beebe, and J P Harbison, Phys Rev Lett 59, 3011 (1987)

${ }^{68} \mathrm{G}$ Timp A M Chang, P Mankiewich, R Behrınger, J E Cunnıngham, T Y Chang, and $R$ E Howard, Phys Rev Lett 59, 732 (1987)

${ }^{69} \mathrm{G}$ Timp, A M Chang, J E Cunningham, T Y Chang, P Mankıewich, R Behringer, and R E Howard, Phys Rev Lett 58, 2814 (1987)

${ }^{70} \mathrm{~A}$ M Chang, G Timp, $\mathrm{T}$ Y Chang, J E Cunnıngham, P M Mankiewich, R E Behrınger, and R E Howard, Solld State Comm 67, 769 (1988)

${ }^{71} \mathrm{~K}$ Y Lee, T P Smith, III, C J B Ford, W Hansen, C M Knoedler, J M Hong, and D P Kern, Appl Phys Lett 55, 625 (1989)

${ }^{72} \mathrm{~J}$ H Davies and J A Nixon, Phys Rev B 39, 3423 (1989), J H Davies, in Ref 15

${ }^{73} \mathrm{C}$ J B Ford, $\mathrm{T}$ J Thornton, R Newbury, M Pepper, H Ahmed, G J Davies, and D Andrews, Superlattices and Microstructures 4, 541 (1988)

${ }^{74} \mathrm{C}$ J B Ford, T J Thornton, R Newbury, M Pepper, $\mathrm{H}$ Ahmed, C T Foxon, J J Harris, and C Roberts, $J$ Phys $C$ 21, L325 (1988)

${ }^{75}$ T P Smith III, H Arnot, J M Hong, C M Knoedler, S E Laux, and H Schmid, Phys Rev Lett 59, $2802(1987)$

${ }^{76}$ T P Smith, III, J A Brum, J M Hong, C M Knoedler, H Arnot, and L Esak1, Phys Rev Lett 61, 585 (1988)

${ }^{77} \mathrm{C}$ J B Ford, S Washburn, M Buttıker, C M Knoedler, and J M Hong, Phys Rev Lett 62, 2724 (1989) 


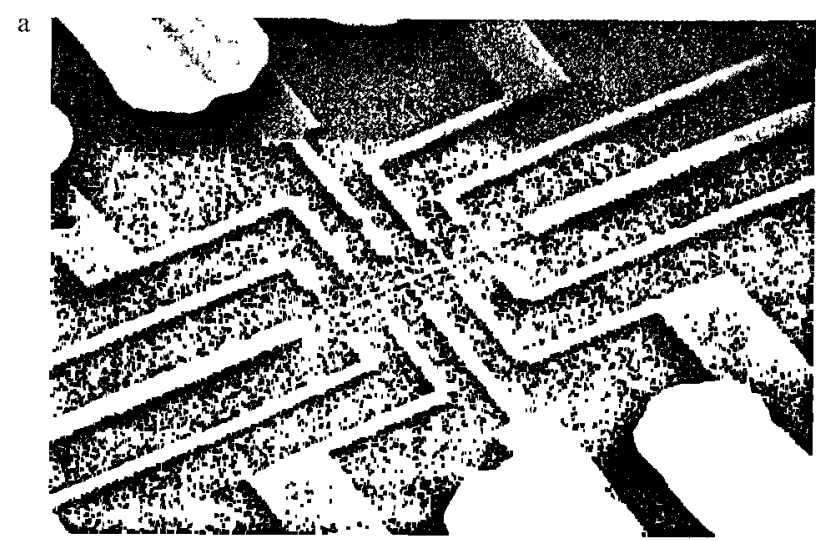

FIG. 5. Scanning electron micrographs of nanostructures in GaAs-AlGaAs heterostructures. (a) Narrow channel (width $75 \mathrm{~nm}$ ), fabricated by means of the confinement scheme of Fig. $4 \mathrm{c}$. The channel has side branches (at a $2-\mu \mathrm{m}$ separation) that serve as voltage probes. Taken from $M$. $L$. Roukes et al., Phys. Rev. Lett. 59, 3011 (1987). (b) Double quantum point contact device, based on the confinement scheme of Fig. $4 \mathrm{~b}$. The bar denotes a length of $1 \mu \mathrm{m}$. Taken from $\mathrm{H}$. van Houten et al., Phys. Rev. B 39, 8556 (1989).

developed by Hansen et al., ${ }^{78,79}$ primarily for the study of one-dimensional subband structure using infrared spectroscopy. Instead of electron beam lithography, they employ a photolithographic technique to define a pattern in the insulator. An array with a very large number of narrow lines is obtained by projecting the interference pattern of two laser beams onto light-sensitive resist. This technique is known as holographic illumination (see Section 11b).

As two representative examples of state-of-the-art nanostructures, we show in Fig. 5a a miniaturized Hall bar, ${ }^{67}$ fabricated by a shallow-mesa etch, and in Fig. 5 b a double-quantum-point contact device, ${ }^{80}$ fabricated by means of the split-gate technique.

Other techniques have been used as well to fabricate narrow electron gas channels. We mention selective-area ion implantation using focused ion beams, ${ }^{81}$ masked ion beam exposure, ${ }^{82}$ strain-induced confinement, ${ }^{83}$ lateral

\footnotetext{
${ }^{78}$ W. Hansen, M. Horst, J. P. Kotthaus, U. Merkt, Ch. Sikorski, and K. Ploog, Phys. Rev. Lett. 58, 2586 (1987).

${ }^{79}$ F. Brinkop, W. Hansen, J. P. Kotthaus, and K. Ploog, Phys. Rev. B 37, 6547 (1988).

${ }^{80}$ H. van Houten, C. W. J. Beenakker, J. G. Williamson, M. E. I. Broekaart, P. H. M. van Loosdrecht, B. J. van Wees, J. E. Mooij, C. T. Foxon, and J. J. Harris, Phys. Rev. B 39, 8556 (1989).

${ }^{81}$ T. Hiramoto, K. Hirakawa, Y. Iye, and T. Ikoma, Appl. Phys. Lett. 54, 2103 (1989).

${ }^{82}$ T. L. Cheeks, M. L. Roukes, A. Scherer, and H. G. Graighead, Appl. Phys. Lett. 53, 1964 (1988).

${ }^{83}$ K. Kash, J. M. Worlock, M. D. Sturge, P. Grabbe, J. P. Harbison, A. Scherer, and P. S. D. Lin, Appl. Phys. Lett. 53, 782 (1988).
} 
b

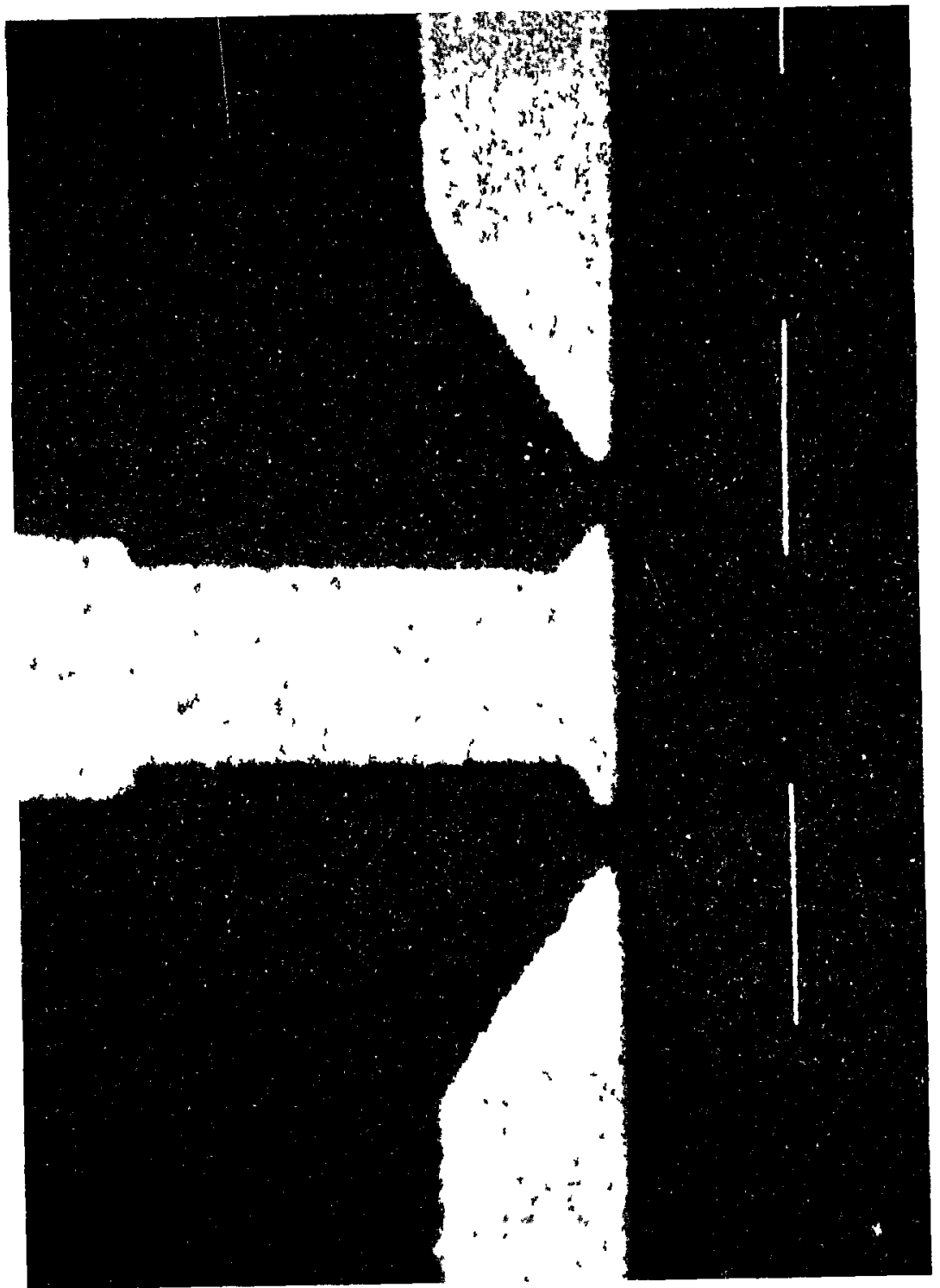


$p-n$ junctions, ${ }^{8485}$ gates in the plane of the $2 \mathrm{DEG}^{86}$ and selective epitaxial growth ${ }^{87-92}$ For more detalled and complete accounts of nanostructure fabrication techniques, we refer to Refs 9 and 13-15

\section{BastC Properties}

\section{a Density of States in Two, One, and Zero Dimensions}

The energy of conduction electrons in a single subband of an unbounded $2 \mathrm{DEG}$, relative to the bottom of that subband, is given by

$$
E(k)=\hbar^{2} k^{2} / 2 m,
$$

as a function of momentum $\hbar k$ The effective mass $m$ is considerably smaller than the free electron mass $m_{\mathrm{e}}$ (see Table I), as a result of interactions with the lattice potential (The incorporation of this potential into an effective mass is an approximation ${ }^{20}$ that is completely justıfied for the present purposes ) The density of states $\rho(E) \equiv d n(E) / d E$ is the derivative of the number of electronic states $n(E)$ (per unit surface area) with energy smaller than $E$ In $k$-space, these states are contained within a curcle of area $A=2 \pi m E / h^{2}$ [according to Eq (41)], which contains a number $g_{\mathrm{s}} g_{\mathrm{v}} A /(2 \pi)^{2}$ of distinct states The factors $g_{\mathrm{s}}$ and $g_{\mathrm{v}}$ account for the spin degeneracy and valley degeneracy, respectıvely (Table I) One thus finds that $n(E)=g_{\mathrm{s}} g_{\mathrm{v}} m E / 2 \pi \hbar^{2}$, so the density of states corresponding to a single subband in a $2 \mathrm{DEG}$,

$$
\rho(E)=g_{\mathrm{s}} g_{\mathrm{v}} m / 2 \pi \hbar^{2},
$$

is independent of the energy As 1llustrated in Fig 6a, a sequence of subbands is associated with the set of discrete levels in the potential well that confines the 2DEG to the interface At zero temperature, all states are filled up to the Fermi energy $E_{\mathrm{F}}$ (this remains a good approximation at finite temperature if the thermal energy $k_{\mathrm{B}} T \ll E_{\mathrm{F}}$ ) Because of the constant density of states, the electron (sheet) density $n_{\mathrm{s}}$ is linearly related to $E_{\mathrm{F}}$ by $n_{\mathrm{s}}=E_{\mathrm{F}} g_{\mathrm{s}} g_{\mathrm{v}} m / 2 \pi \hbar^{2}$ The Fermı wave number $k_{\mathrm{F}} \equiv\left(2 m E_{\mathrm{F}} / \hbar^{2}\right)^{1 / 2}$ is thus related to the density by $k_{\mathrm{F}}=\left(4 \pi n_{\mathrm{s}} / g_{\mathrm{s}} g_{\mathrm{v}}\right)^{1 / 2}$ The second subband starts to be populated when $E_{\mathrm{F}}$ exceeds the energy of the second band bottom The stepwise increasing

\footnotetext{
${ }^{84} \mathrm{U}$ Meirav, M Heıblum, and F Stern, Appl Phys Lett 52, 1268 (1988)

${ }^{85}$ U Meirdv M A Kastner, M Heiblum, and S J Wind, Phys Rev B 40, 5871 (1989)

${ }^{86}$ A D Wleck and K Ploog, Surf Sci 229, 252 (1990), Appl Phys Lett 56, 928 (1990)

${ }^{87} \mathrm{P}$ M Petroff, A C Gossard, and W Wregmann, Appl Phys Lett 45, 620 (1984)

${ }^{88} \mathrm{~T}$ Fukui and H Salto Appl Phys Lett 50, 824 (1987)

${ }^{89} \mathrm{H}$ Asdi, S Yamada, and T Fuku1 Appl Phys Lett 51, 1518 (1987)

${ }^{90}$ T Fuku1, and H Satto, $J$ Vac Sct Technol B6, 1373 (1988)

${ }^{91} \mathrm{~J}$ Motohisa, M Tanaka, and H Sakaki, Appl Phys Lett 55, 1214 (1989)

${ }^{92} \mathrm{H}$ Yamaguchı and Y Horikoshi, Jpn J Appl Phys 28, L1456 (1989)
} 

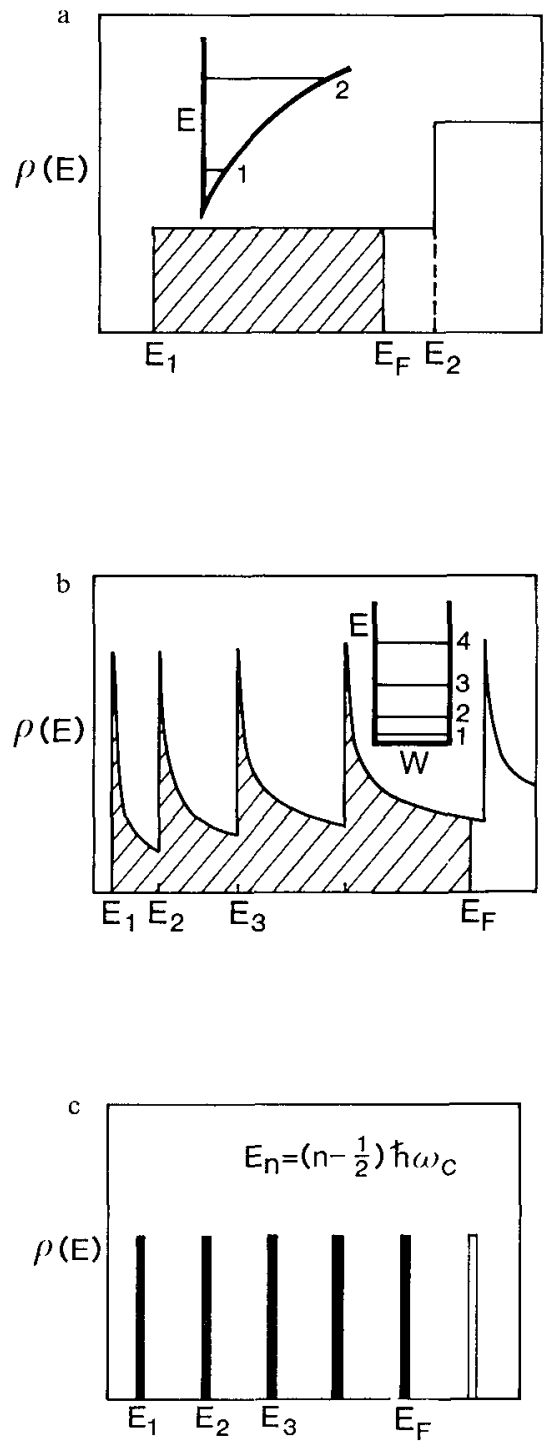

FIG. 6. Density of states $\rho(E)$ as a function of energy. (a) Quasi-2D density of states, with only the lowest subband occupied (hatched). Inset: Confinement potential perpendicular to the plane of the 2DEG. The discrete energy levels correspond to the bottoms of the first and second 2D subbands. (b) Quasi-1D density of states, with four 1D subbands occupied. Inset: Square-well lateral confinement potential with discrete energy levels indicating the 1D subband bottoms. (c) Density of states for a 2DEG in a perpendicular magnetic field. The occupied OD subbands or Landau levels are shown in black. Impurity scattering may broaden the Landau levels, leading to a nonzero density of states between the peaks. 
density of states shown in Fig. 6a is referred to as quasi-two-dimensional. As the number of occupied subbands increases, the density of states eventually approaches the $\sqrt{E}$ dependence characteristic for a three-dimensional system. Note, however, that usually only a single subband is occupied.

If the 2DEG is confined laterally to a narrow channel, then Eq. (4.1) only represents the kinetic energy from the free motion (with momentum $\hbar k$ ) parallel to the channel axis. Because of the lateral confinement, a single twodimensional (2D) subband is split itself into a series of one-dimensional (1D) subbands, with band bottoms at $E_{n}, n=1,2, \ldots$ The total energy $E_{n}(k)$ of an electron in the $n$th $1 \mathrm{D}$ subband (relative to the bottom of the 2D subband) is given by

$$
E_{n}(k)=E_{n}+\hbar^{2} k^{2} / 2 m .
$$

Two frequently used potentials to model analytically the lateral confinement are the square-well potential (of width $W$, illustrated in Fig. 6b) and the parabolic potential well (described by $V(x)=\frac{1}{2} m \omega_{0}^{2} x^{2}$ ). The confinement levels are then given either by $E_{n}=(n \pi \hbar)^{2} / 2 m W^{2}$ for the square well or by $E_{n}=\left(n-\frac{1}{2}\right) \hbar \omega_{0}$ for the parabolic well. When one considers electron transport through a narrow channel, it is useful to distinguish between states with positive and negative $k$, since these states move in opposite directions along the channel. We denote by $\rho_{n}^{+}(E)$ the density of states with $k>0$ per unit channel length in the $n$th $1 \mathrm{D}$ subband. This quantity is given by

$$
\rho_{n}^{+}(E)=g_{\mathrm{s}} g_{\mathrm{v}}\left(2 \pi \frac{d E_{n}(k)}{d k}\right)^{-1}=g_{\mathrm{s}} g_{\mathrm{v}} \frac{m}{2 \pi \hbar^{2}}\left(\frac{\hbar^{2}}{2 m\left(E-E_{n}\right)}\right)^{1 / 2} .
$$

The density of states $\rho_{n}^{-}$with $k<0$ is identical to $\rho_{n}^{+}$. (This identity holds because of time-reversal symmetry; In a magnetic field, $\rho_{n}^{+} \neq \rho_{n}^{-}$, in general.) The total density of states $\rho(E)$, drawn in Fig. 6b, is twice the result (4.4) summed over all $n$ for which $E_{n}<E$. The density of states of a quasi-onedimensional electron gas with many occupied 1D subbands may be approximated by the $2 \mathrm{D}$ result (4.2).

If a magnetic field $B$ is applied perpendicular to an unbounded 2DEG, the energy spectrum of the electrons becomes fully discrete, since free translational motion in the plane of the $2 \mathrm{DEG}$ is impeded by the Lorentz force. Quantization of the circular cyclotron motion leads to energy levels at ${ }^{93}$

$$
E_{n}=\left(n-\frac{1}{2}\right) \hbar \omega_{\mathrm{c}},
$$

with $\omega_{\mathrm{c}}=e B / m$ the cyclotron frequency. The quantum number $n=1,2, \ldots$ labels the Landau levels. The number of states is the same in each Landau level and equal to one state (for each spin and valley) per flux quantum $h / e$

\footnotetext{
${ }^{93}$ L. D. Landau and E. M. Lifshitz, "Quantum Mechanics." Pergamon, Oxford, 1977.
} 
through the sample. To the extent that broadening of the Landau levels by disorder can be neglected, the density of states (per unit area) can be approximated by

$$
\rho(E)=g_{\mathrm{s}} g_{\mathrm{v}} \frac{e B}{h} \sum_{n=1}^{\infty} \delta\left(E-E_{n}\right)
$$

as illustrated in Fig. 6c. The spin degeneracy contained in Eq. (4.6) is resolved in strong magnetic fields as a result of the Zeeman splitting $g \mu_{\mathrm{B}} B$ of the Landau levels ( $\mu_{\mathrm{b}} \equiv e \hbar / 2 m_{\mathrm{e}}$ denotes the Bohr magneton; the Lande $g$-factor is a complicated function of the magnetic field in these systems). ${ }^{20}$ Again, if a large number of Landau levels is occupied (i.e., at weak magnetic fields), one recovers approximately the $2 \mathrm{D}$ result (4.2). The foregoing considerations are for an unbounded 2DEG. A magnetic field perpendicular to a narrow 2DEG channel causes the density of states to evolve gradually from the $1 \mathrm{D}$ form of Fig. $6 \mathrm{~b}$ to the effectively $\mathrm{OD}$ form of Fig. $6 \mathrm{c}$. This transition is discussed in Section 10.

\section{b. Drude Conductivity, Einstein Relation, and Landauer Formula}

In the presence of an electric field $E$ in the plane of the 2DEG, an electron acquires a drift velocity $\mathbf{v}=-e \mathbf{E} \Delta t / m$ in the time $\Delta t$ since the last impurity collision. The average of $\Delta t$ is the scattering time $\tau$, so the average drift velocity $\mathbf{v}_{\text {drift }}$ is given by

$$
\mathbf{v}_{\text {drift }}=-\mu_{\mathrm{e}} \mathbf{E}, \quad \mu_{\mathrm{e}}=e \tau / m .
$$

The electron mobility $\mu_{\mathrm{e}}$ together with the sheet density $n_{\mathrm{s}}$ determine the conductivity $\sigma$ in the relation $-e n_{s} \mathbf{v}_{\text {drift }}=\sigma \mathbf{E}$. The result is the familiar Drude conductivity, ${ }^{94}$ which can be written in several equivalent forms:

$$
\sigma=e n_{\mathrm{s}} \mu_{\mathrm{e}}=\frac{e^{2} n_{\mathrm{s}} \tau}{m}=g_{\mathrm{s}} g_{\mathrm{v}} \frac{e^{2}}{h} \frac{k_{\mathrm{F}} l}{2}
$$

In the last equality we have used the identity $n_{\mathrm{s}}=g_{\mathrm{s}} g_{\mathrm{v}} k_{\mathrm{F}}^{2} / 4 \pi$ (see Section $4 \mathrm{a}$ ) and have defined the mean free path $l=v_{\mathrm{F}} \tau$. The dimensionless quantity $k_{\mathrm{F}} l$ is much greater than unity in metallic systems (see Table I for typical values in a $2 \mathrm{DEG}$ ), so the conductivity is large compared with the quantum unit $e^{2} / h \approx(26 \mathrm{k} \Omega)^{-1}$.

From the preceding discussion it is obvious that the current induced by the applied electric field is carried by all conduction electrons, since each electron acquires the same average drift velocity. Nonetheless, to determine the conductivity it is sufficient to consider the response of electrons near the

\footnotetext{
${ }^{94}$ N. W. Ashcroft and N. D. Mermin, "Solid State Physics." Holt, Rinehart and Winston, New York, 1976.
} 
Fermi level to the electric field. The reason is that the states that are more than a few times the thermal energy $k_{\mathrm{B}} T$ below $E_{\mathrm{F}}$ are all filled so that in response to a weak electric field only the distribution of electrons among states at energies close to $E_{\mathrm{F}}$ is changed from the equilibrium Fermi-Dirac distribution

$$
f\left(E-E_{\mathrm{F}}\right)=\left(1+\exp \frac{E-E_{\mathrm{F}}}{k_{\mathrm{B}} T}\right)^{-1} .
$$

The Einstein relation ${ }^{94}$

$$
\sigma=e^{2} \rho\left(E_{\mathrm{F}}\right) D
$$

is one relation between the conductivity and Fermi level properties (in this case the density of states $\rho(E)$ and the diffusion constant $D$, both evaluated at $E_{\mathrm{F}}$ ). The Landauer formula ${ }^{4}$ [Eq. (4.21)] is another such relation (in terms of the transmission probability at the Fermi level rather than in terms of the diffusion constant).

The Einstein relation (4.10) for an electron gas at zero temperature follows on requiring that the sum of the drift current density $-\sigma \mathbf{E} / e$ and the diffusion current density $-D \nabla n_{\mathrm{s}}$ vanishes in thermodynamic equilibrium, characterized by a spatially constant electrochemical potential $\mu$ :

$$
-\sigma \mathbf{E} / e-D \nabla n_{\mathrm{s}}=0, \quad \text { when } \nabla \mu=0 .
$$

The electrochemical potential is the sum of the electrostatic potential energy $-e V$ (which determines the energy of the bottom of the conduction band) and the chemical potential $E_{\mathrm{F}}$ (being the Fermi energy relative to the conduction band bottom). Since (at zero temperature) $d E_{\mathrm{F}} / d n_{\mathrm{s}}=1 / \rho\left(E_{\mathrm{F}}\right)$, one has

$$
\nabla \mu=e \mathbf{E}+\rho\left(E_{\mathrm{F}}\right)^{-1} \nabla n_{\mathrm{s}} .
$$

The combination of Eqs. (4.11) and (4.12) yields the Einstein relation (4.10) between $\sigma$ and $D$. To verify that Eq. (4.10) is consistent with the earlier expression (4.8) for the Drude conductivity, one can use the result (see below) for the 2D diffusion constant:

$$
D=\frac{1}{2} v_{\mathrm{F}}^{2} \tau=\frac{1}{2} v_{\mathrm{F}} l,
$$

in combination with Eq. (4.2) for the 2D density of states.

At a finite temperature $T$, a chemical potential (or Fermi energy) gradient $\nabla E_{\mathrm{F}}$ induces a diffusion current that is smeared out over an energy range of order $k_{\mathrm{B}} T$ around $E_{\mathrm{F}}$. The energy interval between $E$ and $E+d E$ contributes to the diffusion current density $\mathbf{j}$ an amount $d \mathbf{j}$ given by

$$
d \mathbf{j}_{\mathrm{diff}}=-D \nabla\left\{\rho(E) f\left(E-E_{\mathrm{F}}\right) d E\right\}=-d E D \rho(E) \frac{d f}{d E_{\mathrm{F}}} \nabla E_{\mathrm{F}},
$$


where the diffusion constant $D$ is to be evaluated at energy $E$. The total diffusion current density follows on integration over $E$ :

$$
\mathbf{j}=-\nabla E_{\mathrm{F}} e^{-2} \int_{0}^{\infty} d E \sigma(E, 0) \frac{d f}{d E_{\mathrm{F}}},
$$

with $\sigma(E, 0)$ the conductivity $(4.10)$ at temperature zero for a Fermi energy equal to $E$. The requirement of vanishing current for a spatially constant electrochemical potential implies that the conductivity $\sigma\left(E_{F}, T\right)$ at temperature $T$ and Fermi energy $E_{\mathrm{F}}$ satisfies $\sigma\left(E_{\mathrm{F}}, T\right) e^{-2} \nabla E_{\mathrm{F}}+\mathbf{j}=0$. Therefore, the finite-temperature conductivity is given simply by the energy average of the zero-temperature result

$$
\sigma\left(E_{\mathrm{F}}, T\right)=\int_{0}^{\infty} d E \sigma(E, 0) \frac{d f}{d E_{\Gamma}}
$$

As $T \rightarrow 0$, $d f / d E_{\mathrm{F}} \rightarrow \delta\left(E-E_{\mathrm{F}}\right)$, so indeed only $E=E_{\mathrm{F}}$ contributes to the energy average. Result (4.16) contains exclusively the effects of a finite temperature that are due to the thermal smearing of the Fermi-Dirac distribution. A possible temperature dependence of the scattering processes is not taken into account.

We now want to discuss one convenient way to calculate the diffusion constant (and hence obtain the conductivity). Consider the diffusion current density $j_{x}$ due to a small constant density gradient, $n(x)=n_{0}+c x$. We write

$$
\begin{aligned}
j_{x} & =\lim _{\Delta t \rightarrow \infty}\left\langle v_{x}(t=0) n(x(t=-\Delta t))\right\rangle=\lim _{\Delta t \rightarrow \infty} c\left\langle v_{x}(0) x(-\Delta t)\right\rangle \\
& =\lim _{\Delta t \rightarrow \infty}-c \int_{0}^{\Delta t} d t\left\langle v_{x}(0) v_{x}(-t)\right\rangle,
\end{aligned}
$$

where $t$ is time and the brackets $\langle\cdots\rangle$ denote an isotropic angular average over the Fermi surface. The time interval $\Delta t \rightarrow \infty$, so the velocity of the electron at time 0 is uncorrelated with its velocity at the earlier time $-\Delta t$. This allows us to neglect at $x(-\Delta t)$ the small deviations from an isotropic velocity distribution induced by the density gradient [which could not have been neglected at $x(0)]$. Since only the time difference matters in the velocity correlation function, one has $\left\langle v_{x}(0) v_{x}(-t)\right\rangle=\left\langle v_{x}(t) v_{x}(0)\right\rangle$. We thus obtain for the diffusion constant $D=-j_{x} / c$ the familiar linear response formula ${ }^{95}$

$$
D=\int_{0}^{\infty} d t\left\langle v_{x}(t) v_{x}(0)\right\rangle
$$

Since, in the semiclassical relaxation time approximation, each scattering event is assumed to destroy all correlations in the velocity, and since a

${ }^{95}$ R. Kubo, M. Toda, and N. Hashıtsume, "Statıstıcal Physıcs II." Springer, Berlın, 1985. 
fraction $\exp (-t / \tau)$ of the electrons has not been scattered in a time $t$, one has (in 2D)

$$
\left\langle v_{x}(t) v_{x}(0)\right\rangle=\left\langle v_{x}(0)^{2}\right\rangle \mathrm{e}^{-t / \tau}=\frac{1}{2} v_{\mathrm{F}}^{2} \mathrm{e}^{-t / \tau} .
$$

Substituting this correlation function for the integrand in Eq. (4.18), one recovers on integration the diffusion constant (4.13).

The Drude conductivity (4.8) is a semiclassical result, in the sense that while the quantum mechanical Fermi-Dirac statistic is taken into account, the dynamics of the electrons at the Fermi level is assumed to be classical. In Section II we will discuss corrections to this result that follow from correlations in the diffusion process due to quantum interference. Whereas for classical diffusion correlations disappear on the time scale of the scattering time $\tau$ [as expressed by the correlation function (4.19)], in quantum diffusion correlations persist up to times of the order of the phase coherence time. The latter time $\tau_{\phi}$ is associated with inelastic scattering and at low temperatures can become much greater than the time $\tau$ associated with elastic scattering.

In an experiment one measures a conductance rather than a conductivity. The conductivity $\sigma$ relates the local current density to the electric field, $j=\sigma E$, while the conductance $G$ relates the total current to the voltage drop, $I=G V$. For a large homogeneous conductor the difference between the two is not essential, since Ohm's law tells us that

$$
G=(W / L) \sigma
$$

for a 2DEG of width $W$ and length $L$ in the current direction. (Note that $G$ and $\sigma$ have the same units in two dimensions.) If for the moment we disregard the effects of phase coherence, then the simple scaling (4.20) holds provided both $W$ and $L$ are much larger than the mean free path $l$. This is the diffusive transport regime, illustrated in Fig. 7a. When the dimensions of the sample are reduced below the mean free path, one enters the ballistic transport regime, shown in Fig. 7c. One can further distinguish an intermediate quasiballistic regime, characterized by $W<l<L$ (see Fig. 7b). In ballistic transport only the conductance plays a role, not the conductivity. The Landauer formula

$$
G=\left(e^{2} / h\right) T
$$

plays a central role in the study of ballistic transport because it expresses the conductance in terms of a Fermi level property of the sample (the transmission probability $T$, see Section 12). Equation (4.21) can therefore be applied to situations where the conductivity does not exist as a local quantity, as we will discuss in Sections III and IV.

If phase coherence is taken into account, then the minimal length scale 
a)

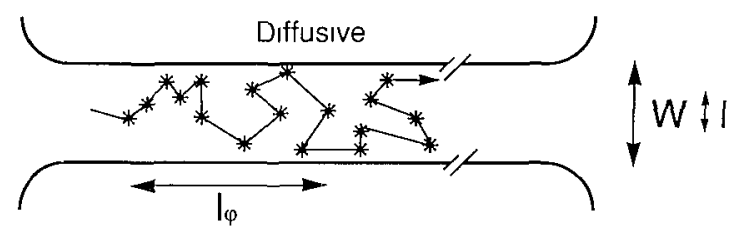

b)
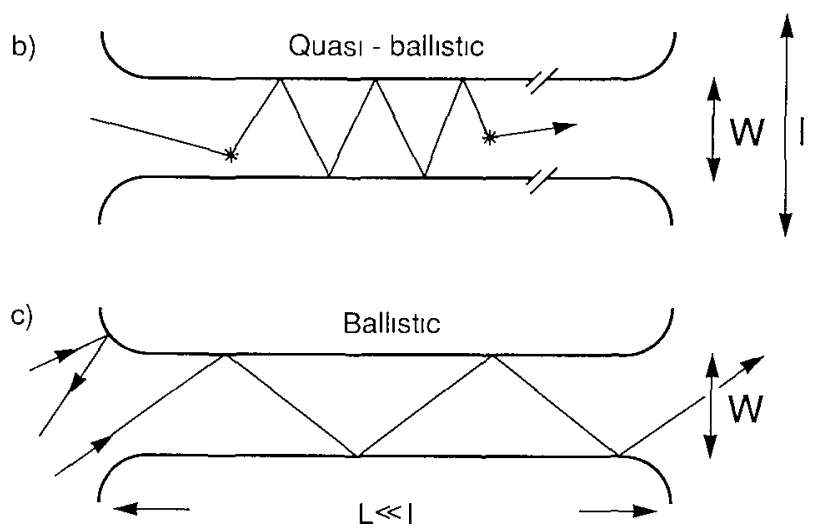

FIG 7. Electron trajectories characteristic for the diffusive $(l<W, L)$, quast-ballistic $(W<l<L)$, and ballistic $(W, L<l)$ transport regimes, for the case of specular boundary scatterıng Boundary scatterıng and internal impurity scattering (asterısks) are of equal importance in the quasi-ballstic regime A nonzero resistance in the ballistic regime results from backscattering at the connection between the narrow channel and the wide 2DEG regions Taken from $\mathbf{H}$ van Houten et al, in "Physics and Technology of Submicron Structures" ( $H$ Heinrıch, G Bauer, and F Kuchar, eds) Sprınger, Berlın, 1988

required to characterize the conductivity becomes larger. Instead of the (elastic) mean free path $l \equiv v_{\mathrm{F}} \tau$, the phase coherence length $l_{\phi} \equiv\left(D \tau_{\phi}\right)^{1 / 2}$ becomes this characteristic length scale (up to a numerical coefficient $l_{\phi}$ equals the average distance that an electron diffuses in the time $\tau_{\phi}$ ). Ohm's law can now only be applied to add the conductances of parts of the sample with dimensions greater than $l_{\phi}$. Since at low temperatures $l_{\phi}$ can become quite large (cf. Table I), it becomes possible that (for a small conductor) phase coherence extends over a large part of the sample. Then only the conductance (not the conductivity) plays a role, even if the transport is fully in the diffusive regime. We will encounter such situations repeatedly in Section II.

\section{c. Magnetotransport}

In a magnetic field $B$ perpendicular to the 2DEG, the current is no longer in the direction of the electric field due to the Lorentz force. Consequently, 
the conductivity is no longer a scalar but a tensor $\sigma$, related via the Einstein relation $\boldsymbol{\sigma}=e^{2} \rho\left(E_{\mathrm{F}}\right) \mathbf{D}$ to the diffusion tensor

$$
\mathbf{D}=\int_{0}^{\infty} d t\langle\mathbf{v}(t) \mathbf{v}(0)\rangle
$$

Equation (4.22) follows from a straightforward generalization of the argument leading to the scalar relation (4.18) [but now the ordering of $\mathbf{v}(t)$ and $\mathbf{v}(0)$ matters]. Between scattering events the electrons at the Fermi level execute circular orbits, with cyclotron frequency $\omega_{\mathrm{c}}=e B / m$ and cyclotron radius $l_{\text {cycl }}=m v_{\mathrm{F}} / e B$. Taking the $2 \mathrm{DEG}$ in the $x-y$ plane, and the magnetic field in the positive $z$-direction, one can write in complex number notation

$$
\tilde{v}(t) \equiv v_{x}(t)+i v_{y}(t)=v_{\mathrm{F}} \exp \left(i \phi+i \omega_{\mathrm{c}} t\right) .
$$

The diffusion tensor is obtained from

$$
D_{x x}+i D_{y x}=\int_{0}^{2 \pi} \frac{d \phi}{2 \pi} \int_{0}^{\infty} d t \tilde{v}(t) v_{\mathrm{F}} \cos \phi \mathrm{e}^{-t / \tau}=\frac{D}{1+\left(\omega_{\mathrm{c}} \tau\right)^{2}}\left(1+i \omega_{\mathrm{c}} \tau\right)
$$

where $D$ is the zero-field diffusion constant (4.13). One easily verifies that $D_{y y}=D_{x x}$ and $D_{x y}=-D_{y x}$. From the Einstein relation one then obtains the conductivity tensor

$$
\sigma=\frac{\sigma}{1+\left(\omega_{\mathrm{c}} \tau\right)^{2}}\left(\begin{array}{cc}
1 & -\omega_{\mathrm{c}} \tau \\
\omega_{\mathrm{c}} \tau & 1
\end{array}\right)
$$

with $\sigma$ the zero-field conductivity (4.8). The resistivity tensor $\rho \equiv \sigma^{-1}$ has the form

$$
\rho=\rho\left(\begin{array}{cc}
1 & \omega_{\mathrm{c}} \tau \\
-\omega_{\mathrm{c}} \tau & 1
\end{array}\right)
$$

with $\rho=\sigma^{-1}=m / n_{\mathrm{s}} e^{2} \tau$ the zero-field resistivity.

The off-diagonal element $\rho_{x y} \equiv R_{\mathrm{H}}$ is the classical Hall resistance of a 2DEG:

$$
R_{\mathrm{H}}=\frac{B}{n_{\mathrm{s}} e}=\frac{1}{g_{\mathrm{s}} g_{\mathrm{v}}} \frac{h}{e^{2}} \frac{h \omega_{\mathrm{c}}}{E_{\mathrm{F}}} .
$$

Note that in a 2D channel geometry there is no distinction between the Hall resistivity and the Hall resistance, since the ratio of the Hall voltage $V_{\mathrm{H}}=W E_{x}$ across the channel to the current $I=W j_{y}$ along the channel does not depend on its length and width (provided transport remains in the diffusive regime). The diagonal element $\rho_{x x}$ is referred to as the longitudinal resistivity. Equation (4.26) tells us that classically the magnetoresistivity is zero (i.e., $\rho_{x x}(B)-\rho_{x x}(0)=0$ ). This counterintuitive result can be understood by considering that the force from the Hall voltage cancels the average 


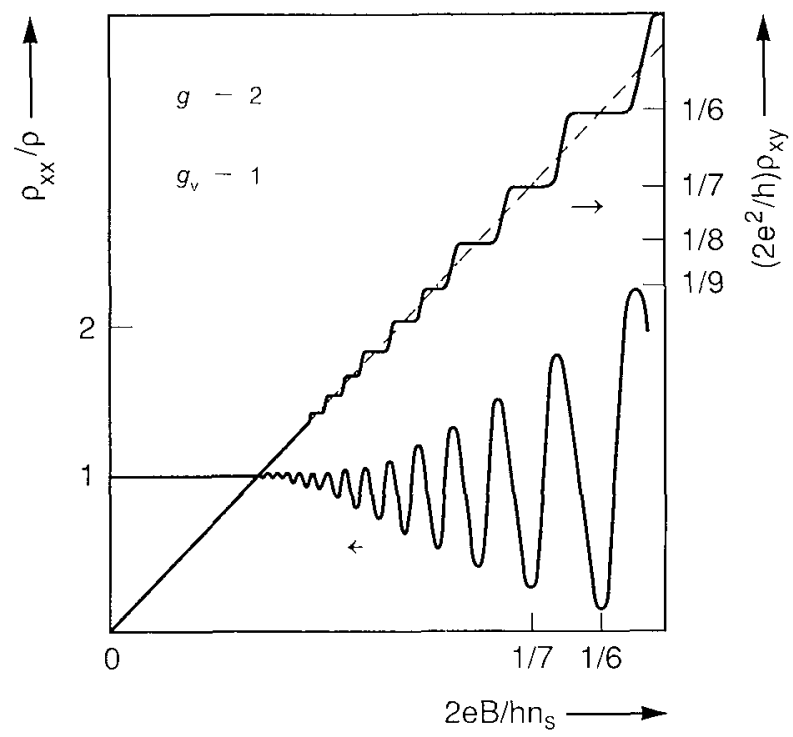

FIG 8 Schematic dependence on the reciprocal filling factor $v^{-1} \equiv 2 e B / h n_{s}$ of the long1tudinal resistivity $\rho_{x}$ (normalized to the zero-field resistivity $\rho$ ) and of the Hall resistance $\mathbf{R}_{\mathbf{H}} \equiv \rho_{x y}$ (normalized to $h / 2 e^{2}$ ) The plot is for the case of a single valley with twofold spin degeneracy Deviations from the semiclassical result (426) occur in strong magnetic fields, in the form of Shubnikov-De Haas oscillations in $\rho_{x x}$ and quantized plateaus [Eq (430)] in $\rho_{x y}$

Lorentz force on the electrons A general conclusion that one can draw from Eqs $(425)$ and $(426)$ is that the classical effects of a magnetic field are important only if $\omega_{\mathrm{c}} \tau \gtrsim 1$ In such fields an electron can complete several cyclotron orbits before being scattered out of orbit In a high-mobility 2DEG this criterion is met at rather weak magnetic fields (note that $\omega_{\mathrm{c}} \tau=\mu_{\mathrm{e}} B$, and see Table I)

In the foregoing application of the Einstein relation we have used the zerofield density of states Moreover, we have assumed that the scattering time is $B$-independent Both assumptions are justified in weak magnetıc fields, for which $E_{\Gamma} / \hbar \omega_{\mathrm{c}} \gg 1$, but not in stronger fields (cf Table I) As illustrated in Fig 8 , deviations from the semiclassical result (4 26) appear as the magnetic field is increased These deviations take the form of an oscillatory magnetoresistivity (the Shubntkov-De Haas effect) and plateaux in the Hall resistance (the quantum Hall effect) The origin of these two phenomena is the formation of Landau levels by a magnetic field, discussed in Section $4 \mathrm{a}$, that leads to the $B$ dependent density of states (4 6) The main effect is on the scattering rate $\tau^{-1}$, which in a simple (Born) approximation ${ }^{96}$ is proportional to $\rho\left(E_{\mathbf{F}}\right)$

$$
\tau^{-1}=(\pi / \hbar) \rho\left(E_{\mathrm{F}}\right) c_{1} u^{2}
$$

${ }^{96}$ A A Abrikosov, "Fundamentals of the Theory of Metals" North-Holland, Amsterdam, 1988 
Here $c_{1}$ is the areal density of impurities, and the impurity potential is modeled by a $2 \mathrm{D}$ delta function of strength $u$. The diagonal element of the resistivity tensor (4.26) is $\rho_{x x}=\left(m / e^{2} n_{\mathrm{s}}\right) \tau^{-1} \propto \rho\left(E_{\mathrm{F}}\right)$. Oscillations in the density of states at the Fermi level due to the Landau level quantization are therefore observable as an oscillatory magnetoresistivity. One expects the resistivity to be minimal when the Fermi level lies between two Landau levels, where the density of states is smallest. In view of Eq. (4.6), this occurs when the Landau level filling factor $\left.v \equiv\left(n_{\mathrm{s}} / g_{\mathrm{s}} g_{\mathrm{v}}\right) h / e B\right)$ equals an integer $N=1,2$, ... (assuming spin-degenerate Landau levels). The resulting Shubnikov-De Haas oscillations are periodic in $1 / B$, with spacing $\Delta(1 / B)$ given by

$$
\Delta\left(\frac{1}{B}\right)=\frac{e}{h} \frac{g_{\mathrm{s}} g_{\mathrm{v}}}{n_{\mathrm{s}}}
$$

providing a means to determine the electron density from a magnetoresistance measurement. This brief explanation of the Shubnikov-De Haas effect needs refinement, ${ }^{20}$ but is basically correct. The quantum Hall effect, ${ }^{8}$ being the occurrence of plateaux in $R_{\mathrm{H}}$ versus $B$ at precisely

$$
R_{\mathrm{H}}=\frac{1}{g_{\mathrm{s}} g_{\mathrm{v}}} \frac{h}{e^{2}} \frac{1}{N}, \quad N=1,2, \ldots,
$$

is a more subtle effect ${ }^{97}$ to which we cannot do justice in a few lines (see Section 18). The quantization of the Hall resistance is related on a fundamental level to the quantization in zero magnetic field of the resistance of a ballistic point contact. ${ }^{6,7} \mathrm{We}$ will present a unified description of both these effects in Sections 12 and 13.

\section{Diffusive and Quasi-Ballistic Transport}

\section{Classical Size Effects}

In metals, the dependence of the resistivity on the size of the sample has been the subject of study for almost a century. ${ }^{98}$ Because of the small Fermi wave length in a metal, these are classical size effects. Comprehensive reviews of this field have been given by Chambers, ${ }^{99}$ Brändli and Olsen, ${ }^{100}$ Sondheimer, ${ }^{101}$ and, recently, Pippard. ${ }^{102}$ In semiconductor nanostructures both

\footnotetext{
${ }^{97} \mathrm{R}$ E Prange and S M Girvin, eds, The Quantum Hall Effect " Springer, New York, 1987

${ }^{98}$ I. Stone, Phys Rev 6, 1 (1898).

${ }^{99} \mathrm{R}$ G Chambers, in "The Physics of Metals," Vol 1 (J M Ziman, ed) Cambridge University Press, Cambridge, 1969

${ }^{100} \mathrm{G}$ Brandl and J L Olsen, Mater Scl Eng 4, 61 (1969)

${ }^{101} \mathrm{E}$ H Sondhemer, Adv Phys 1, 1 (1952)

${ }^{102}$ A B Pippard, "Magnetoresistance in Metals." Cambridge University Press, Cambridge, 1989
} 
classical and quantum size effects appear, and an understanding of the former is necessary to distinguish them from the latter Classical size effects in a 2DEG are of intrinsic interest as well First of all, a 2DEG is an ideal model system to study known size effects without the complications of nonspherical Fermı surfaces and polycrystallınity, characteristıc for metals Furthermore, it is possible in a $2 \mathrm{DEG}$ to study the case of nearly complete specular boundary scattering, whereas in a metal diffuse scattering dominates The much smaller cyclotron radius in a $2 D E G$, compared with a metal at the same magnetic field value, allows one to enter the regime where the cyclotron radius is comparable to the range of the scattering potential The resulting modifications of known effects in the quası-ballistıc transport regime are the subject of this section A variety of new classical size effects, not known from metals, appear in the ballistic regime, when the resistance is measured on a length scale below the mean free path These are discussed in Section 16, and require a reconsideration of what is meant by a resistance on such a short length scale

In the present section we assume that the channel length $L$ (or, more generally, the separation between the voltage probes) is much larger than the mean free path $l$ for impurity scattering so that the motion remains diffusive along the channel Size effects in the resistivity occur when the motion across the channel becomes ballistic (1 e, when the channel width $W<l$ ) Diffuse boundary scattering leads to an increase in the resistivity in a zero magnetic field and to a nonmonotonic magnetoresistivity in a perpendicular magnetic field, as discussed in the following two subsections The 2D channel geometry is essentrally equivalent to the $3 \mathrm{D}$ geometry of a thın metal plate in a parallel magnetic field, with the current flowing perpendicular to the field Size effects in this geometry were origınally studied by Fuchs ${ }^{103}$ in a zero magnetic field and by MacDonald ${ }^{104}$ for a nonzero field The alternative configuration in which the magnetic field is perpendicular to the thin plate, studied by Sondheimer, ${ }^{105}$ does not have a $2 \mathrm{D}$ analog We discuss in this section only the classical size effects, and thus the discreteness of the $1 \mathrm{D}$ subbands and of the Landau levels is 1gnored Quantum size effects in the quasi-ballistic transport regime are treated in Section 10

\section{a Boundary Scattering}

In a zero magnetic field, scattering at the channel boundaries increases the resistivity, unless the scattering is specular Specular scattering occurs if the confining potential $V(x, y)$ does not depend on the coordinate $y$ along the channel axis In that case the electron motion along the channel is not

\footnotetext{
${ }^{103}$ K Fuchs, Proc Cambridge Philos Soc 34, 100 (1938)

${ }^{104}$ D K C MacDonald, Nature 163, 637 (1949), D K C MacDonald and K Sarginson, Proc Roy Soc A 203, 223 (1950)

${ }^{105}$ E H Sondheimer, Nature 164, 920 (1949), Phys Rev 80, 401 (1950)
} 
influenced at all by the lateral confinement, so the resistivity $\rho$ retains its $2 \mathrm{D}$ bulk value $\rho_{0}=m / e^{2} n_{s} \tau$. More generally, specular scattering requires any roughness of the boundaries to be on a length scale smaller than the Fermi wavelength $\lambda_{\mathrm{F}}$. The confining potential created electrostatically by means of a gate electrode is known to cause predominantly specular scattering (as has been demonstrated by the electron focusing experiments ${ }^{59}$ discussed in Section 14). This is a unique situation, not previously encountered in metals, where as a result of the small $\lambda_{\mathrm{F}}$ (on the order of the interatomic separation) diffuse boundary scattering dominates. ${ }^{102}$

Diffuse scattering means that the velocity distribution at the boundary is isotropic for velocity directions that point away from the boundary. Note that this implies that an incident electron is reflected with a (normalized) angular distribution $P(\alpha)=\frac{1}{2} \cos \alpha$, since the reflection probability is proportional to the flux normal to the boundary. Diffuse scattering increases the resistivity above $\rho_{0}$ by providing an upper bound $W$ to the effective mean free path. In order of magnitude, $\rho \sim(l / W) \rho_{0}$ if $l \gtrsim W$ (a more precise expression is derived later). In general, boundary scattering is neither fully specular nor fully diffuse and, moreover, depends on the angle of incidence (grazing incidence favors specular scattering since the momentum along the channel is large and not easily reversed). The angular dependence is often ignored for simplicity, and the boundary scattering is described, following Fuchs, ${ }^{103}$ by a single parameter $p$, such that an electron colliding with the boundary is reflected specularly with probability $p$ and diffusely with probability $1-p$. This specularity parameter is then used as a fit parameter in comparison with experiments. Soffer ${ }^{106}$ has developed a more accurate, and more complicated, modeling in terms of an angle of incidence dependent specularity parameter.

In the extreme case of fully diffuse boundary scattering $(p=0)$, one is justified in neglecting the dependence of the scattering probability on the angle of incidence. We treat this case here in some detail to contrast it with fully specular scattering, and because diffuse scattering can be of importance in 2DEG channels defined by ion beam exposure rather than by gates. ${ }^{107,108}$ We calculate the resistivity from the diffusion constant by means of the Einstein relation. Fuchs takes the alternative (but equivalent) approach of calculating the resistivity from the linear response to an applied electric field. ${ }^{103}$ Impurity scattering is taken as isotropic and elastic and is described by a scattering time $\tau$ such that an electron is scattered in a time interval $d t$ with probability $d t / \tau$, regardless of its position and velocity. This is the commonly employed "scattering time" (or "relaxation time") approximation.

${ }^{106}$ S. B. Soffer, J. Appl. Phys. 38, 1710 (1967).

${ }^{107}$ T. J. Thornton, M. L. Roukes, A. Scherer, and B. P. van der Gaag, Phys. Rev. Lett. 63, 2128 (1989).

${ }^{108}$ K. Nakamura, D. C. Tsui, F. Nihey, H. Toyoshima, and T. Itoh, Appl. Phys. Lett. 56, 385 (1990). 
The channel geometry is defined by hard walls at $x= \pm W / 2$ at which the electrons are scattered diffusely The stationary electron distribution function at the Fermı energy $F(\mathbf{r}, \alpha)$ satisfies the Boltzmann equation

$$
\mathbf{v} \cdot \frac{\partial}{\partial \mathbf{r}} F=-\frac{1}{\tau} F+\frac{1}{\tau} \int_{0}^{2 \pi} \frac{d \alpha}{2 \pi} F
$$

where $\mathbf{r} \equiv(x, y)$ is the position and $\alpha$ is the angle that the velocity $\mathbf{v} \equiv v_{\mathrm{F}}(\cos \alpha$, $\sin \alpha$ ) makes with the $x$-axis The boundary condition corresponding to diffuse scattering is that $F$ is independent of the velocity direction for velocities pointing away from the boundary In view of current conservation this boundary condition can be written as

$$
\begin{array}{rlrl}
F(\mathbf{r}, \alpha) & =\frac{1}{2} \int_{-\pi / 2}^{\pi / 2} d \alpha^{\prime} F\left(\mathbf{r}, \alpha^{\prime}\right) \cos \alpha^{\prime}, & & \text { for } x=\frac{W}{2}, \frac{\pi}{2}<\alpha<\frac{3 \pi}{2}, \\
& =\frac{1}{2} \int_{\pi / 2}^{3 \pi / 2} d \alpha^{\prime} F\left(\mathbf{r}, \alpha^{\prime}\right) \cos \alpha^{\prime}, & \text { for } x=-\frac{W}{2},-\frac{\pi}{2}<\alpha<\frac{\pi}{2}
\end{array}
$$

To determine the diffusion constant, we look for a solution of Eqs (51) and (52) corresponding to a constant density gradient along the channel, $F(\mathbf{r}, \alpha)=-c y+f(x, \alpha)$ Since there is no magnetic field, we anticipate that the density will be uniform across the channel width so that $\int_{0}^{2 \pi} f d \alpha=0$ The Boltzmann equation (51) then simplifies to an ordinary differential equation for $f$, which can be solved straightforwardly The solution that satısfies the boundary conditions (5 2) is

$$
F(\mathbf{r}, \alpha)=-c y+c l \sin \alpha\left[1-\exp \left(-\frac{W}{2 l|\cos \alpha|}-\frac{x}{l \cos \alpha}\right)\right],
$$

where we have written $l \equiv v_{1} \tau$ One easily verifies that $F$ has indeed a uniform density along $x$ The diffusion current

$$
I_{y}=v_{\mathrm{F}} \int_{W / 2}^{W / 2} d x \int_{0}^{2 \pi} d \alpha F \sin \alpha
$$

along the channel in response to the density gradient $\partial n / \partial y=-2 \pi c$ determines the diffusion constant $D=-\left(I_{y} / W\right)(\partial n / \partial y)^{-1}$ The resistivity $\rho=E_{\mathrm{F}} / n_{\mathrm{s}} e^{2} D$ then follows from the Enstein relation (410), with the $2 \mathrm{D}$ density of states $n_{\mathrm{s}} / E_{\mathrm{F}}$ The resulting expression is

$$
\rho=\rho_{0}\left[1-\frac{4 l}{\pi W} \int_{0}^{1} d \xi \xi\left(1-\xi^{2}\right)^{1 / 2}\left(1-\mathrm{e}^{-W / l \xi}\right)\right]^{-1},
$$

which can be easily evaluated numerically It is worth noting that the above result ${ }^{109}$ for $\rho / \rho_{0}$ in a $2 \mathrm{D}$ channel geometry does not differ much (less than $20 \%$ ) from the corresponding result ${ }^{103}$ in a $3 \mathrm{D}$ thin film

${ }^{109} \mathrm{C}$ W J Beenakker and $\mathrm{H}$ van Houten, Phys Rev B 38, 3232 (1988) 
For $l / W \ll 1$ one has

$$
\rho=\rho_{0}\left(1+\frac{4}{3 \pi} \frac{l}{W}\right)
$$

which differs from $\mathrm{Eq}(55)$ by less than $10 \%$ in the range $l / W \lesssim 10$ For $l / W \gg 1$ one has asymptotically

$$
\rho=\frac{\pi}{2} \rho_{0} \frac{l}{W} \frac{1}{\ln (l / W)}=\frac{\pi}{2} \frac{m v_{\mathrm{F}}}{n_{\mathrm{s}} e^{2} W} \frac{1}{\ln (l / W)}
$$

In the absence of impurity scattering ( $1 \mathrm{e}$, in the limit $l \rightarrow \infty$ ), Eq (5 7) predicts a vanishing resistivity Diffuse boundary scatterıng is ineffectıve in establishing d finite resistivity in this limit, because electrons with velocities nearly parallel to the channel walls can propagate over large distances without collisions and thereby short out the current As shown by Tesanovic et al,${ }^{110}$ a small but nonzero resistivity in the absence of impurity scattering is recovered if one goes beyond the semiclassical approximation and includes the effect of the quantum mechanical uncertainty in the transverse component of the electron velocity

\section{b Magneto Size Effects}

In an unbounded 2DEG, the longitudinal resistivity is magnetic-fieldindependent in the semiclassical approximation (see Section 4c) We will discuss how a nonzero magnetoresistivity can arise classically as a result of boundary scattering We consider the two extreme cases of specular and diffuse boundary scattering, and describe the impurity scattering in the scattering time approximation Shortcomings of this approximation are discussed toward the end of this subsection

We consider first the case of specular boundary scattering In a zero magnetic field it is obvious that specular scattering cannot affect the resistivity, since the projection of the electron motion on the channel axis is not changed by the presence of the channel boundaries If a magnetic field is applied perpendicular to the 2DEG, the electron trajectories in a channel cannot be mapped in this way on the trajectories in an unbounded system In fact, in an unbounded 2DEG in equilibrium the electrons perform closed cyclotron orbits between scattering events, whereas a channel geometry supports open orbits that skip along the boundaries One might suppose that the presence of these skipping orbits propagating along the channel would increase the diffusion constant and hence reduce the (longitudinal) resistivity below the value $\rho_{0}$ of a bulk 2DEG That is not correct, at least in the scattering tıme approximation, as we now demonstrate

${ }^{110} \mathrm{Z}$ Tesanovic, M V Jaric, and S Maekawa, Phys Rev Lett 57, 2760 (1986) 
The stationary Boltzmann equation in a magnetic field $\mathbf{B}$ in the $z$-direction (perpendicular to the 2DEG) is

$$
\mathbf{v} \cdot \frac{\partial}{\partial \mathbf{r}} F+\omega_{\mathrm{c}} \frac{\partial}{\partial \alpha} F=-\frac{1}{\tau} F+\frac{1}{\tau} \int_{0}^{2 \pi} \frac{d \alpha}{2 \pi} F
$$

Here, we have used the identity $-e m^{-1}(\mathbf{v} \times \mathbf{B}) \cdot \partial / \partial \mathbf{v} \equiv \omega_{\mathrm{c}} \partial / \partial \alpha$ (with $\omega_{\mathrm{c}} \equiv e B / m$ the cyclotron frequency) to rewrite the term that accounts for the Lorentz force The distribution function $F(\mathbf{r}, \alpha)$ must satısfy the boundary conditions for specular scattering,

$$
F(\mathbf{r}, \alpha)=F(\mathbf{r}, \pi-\alpha), \quad \text { for } x= \pm W / 2
$$

One readıly verıfies that

$$
F(\mathbf{r}, \alpha)=-c\left(y+\omega_{\mathrm{c}} \tau x\right)+c l \sin \alpha
$$

is a solution of Eqs (58) and (59) The corresponding diffusion current $I_{y}=\pi c W v_{\mathrm{F}} l$ and density gradient along the channel $\partial n / \partial y=-2 \pi c$ are both the same as in a zero magnetic field It follows that the diffusion constant $D=I_{y} / 2 \pi c W$ and, hence, the longitudinal resistivity $\rho=E_{\mathrm{F}} / n_{\mathrm{s}} e^{2} D$ are $B$ independent, that is, $\rho=\rho_{0} \equiv m / n_{\mathrm{s}} e^{2} \tau$ as in an unbounded 2DEG More generally, one can show that in the scattering time approximation the longitudinal resistivity is $B$-independent for any confining potential $V(x, y)$ that does not vary with the coordinate $y$ along the channel axis (This statement is proven by applying the result of Ref 111 , of a $B$-independent $\rho_{y y}$ for periodic $V(x)$, to a set of disjunct parallel channels (see Section 11b), the case of a single channel then follows from Ohm's law )

In the case of diffuse boundary scattering, the zero-field resistivity is enhanced by approximately a factor $1+l / 2 W$ [see Eq (56)] A sufficiently strong magnetic field suppresses this enhancement, and reduces the resistivity to its bulk value $\rho_{0}$ The mechanism for this negative magnetoresistance is illustrated in $\mathrm{F}_{1 \mathrm{~g}} 9 \mathrm{~b}$ If the cyclotron diameter $2 l_{\text {cycl }}$ is smaller than the channel width $W$, diffuse boundary scattering cannot reverse the direction of motion along the channel, as it could for smaller magnetic fields The diffusion current is therefore approximately the same as in the case of specular scattering, in which case we have seen that the diffusion constant and, hence, resistivity have their bulk values Figure 9 represents an example of magnetic reduction of backscattering Recently, this phenomenon has been understood to occur in an extreme form in the quantum Hall effect ${ }^{112}$ and in ballistic transport through quantum point contacts $^{113}$ The effect was

${ }^{111}$ C W J Beenakker, Phys Rev Lett 62, 2020 (1989)

${ }^{112}$ M Butt1ker, Phys Rev B 38, 9375 (1988)

${ }^{113} \mathrm{H}$ van Houten C W J Beenakker, P H M van Loosdrecht, T J Thornton, H Ahmed, M Pepper, C T Foxon, and J J Harrs, Phys Rev B 37, 8534 (1988), and unpublished 
(a)

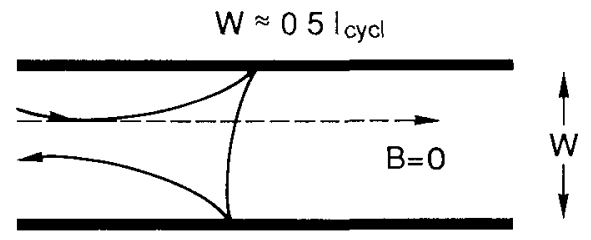

(b)

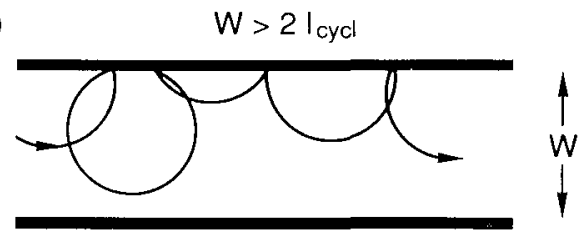

FIG 9 Illustration of the effect of a magnetic field on motion through a channel with diffuse boundary scattering (a) Electrons which in a zero field move nearly parallel to the boundary can reverse their motion in weak magnetic fields This increases the resistivity (b) Suppression of backscattering at the boundaries in strong magnetic fields reduces the resistivity.

essentially known and understood by MacDonald ${ }^{104}$ in 1949 in the course of his magnetoresistivity experiments on sodium wires. The ultimate reduction of the resistivity is preceded by an initial increase in weak magnetic fields, due to the deflection toward the boundary of electrons with a velocity nearly parallel to the channel axis (Fig. 9a). The resulting nonmonotonic $B$ dependence of the resistivity is shown in Fig. 10. The plot for diffuse scattering is based on a calculation by Ditlefsen and Lothe ${ }^{114}$ for a 3D thinfilm geometry. The case of a $2 \mathrm{D}$ channel has been studied by Pippard ${ }^{102}$ in the limit $l / W \rightarrow \infty$, and he finds that the $2 \mathrm{D}$ and $3 \mathrm{D}$ geometries give very similar results.

An experimental study of this effect in a $2 D E G$ has been performed by Thornton et al. ${ }^{107}$ In Fig. 11 their magnetoresistance data are reproduced for channels of different widths $W$, defined by low-energy ion beam exposure. It was found that the resistance reaches a maximum when $W \approx 0.5 l_{\text {cycl }}$, in excellent agreement with the theoretical predictions. ${ }^{14,102}$ Thornton et al. also investigated channels defined electrostatically by a split gate, for which one expects predominantly specular boundary scattering. ${ }^{59}$ The foregoing analysis would then predict an approximately $B$-independent resistance (Fig. 10 ), and indeed only a small resistance maximum was observed in weak magnetic fields. At stronger fields, however, the resistance was found to decrease substantially. Such a monotonically decreasing resistance in 


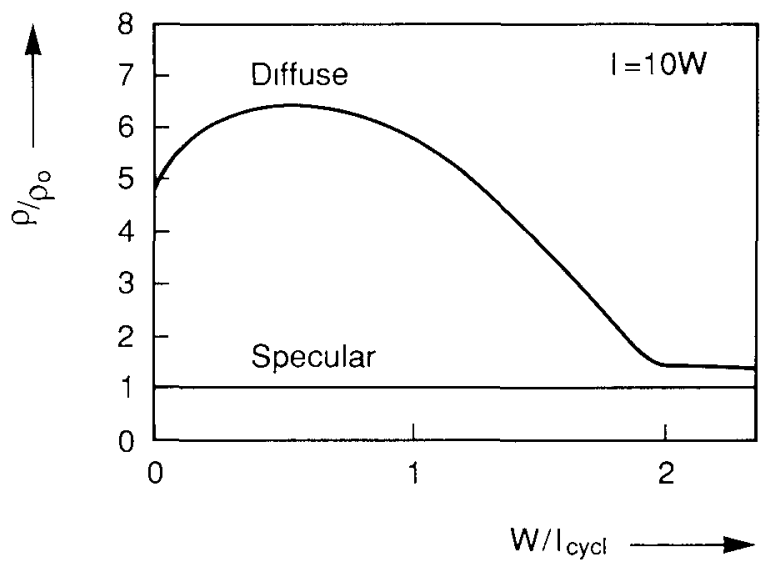

FIG 10 Magnetic field dependence of the longitudinal resistivity of a channel for the two cases of diffuse and specular boundary scattering, obtained from the Boltzmann equation in the scattering time approximation The plot for diffuse scattering is the result of Ref 114 for a 3D thin film geometry with $l=10 \mathrm{~W}$ (A $2 \mathrm{D}$ channel geometry is expected to give very similar results ${ }^{102}$ )

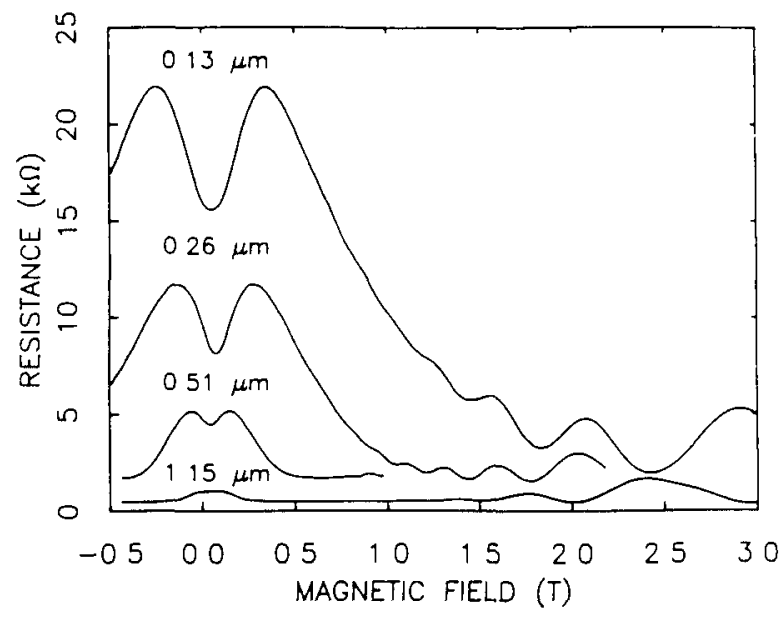

FIG 11 Experimental magnetic field dependence of the resistance of channels of different widths, defined by ion beam exposure in the 2DEG of a GaAs-AlGaAs heterostructure ( $L=12 \mu \mathrm{m}, T=42 \mathrm{~K}$ ) The nonmonotonic magnetic field dependence below $1 \mathrm{~T}$ is a classical size effect due to diffuse boundary scattering, as illustrated in Fig 9 The magnetoresistance oscillations at higher fields result from the quantum mechanical Shubnikov-De Haas effect Taken from T J Thornton et al, Phys Rev Lett 63, 2128 (1989) 
(a)

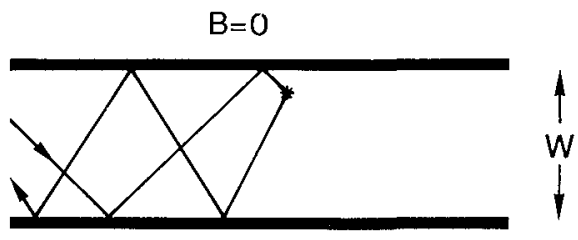

(b)

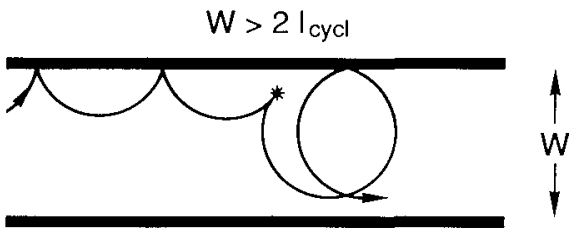

FIG. 12. Electron trajectories in a channel with specular boundary scattering, to illustrate how a magnetic field can suppress the backscattering by an isolated impurity close to a boundary. This effect would lead to a negative magnetoresistivity if one would go beyond the scattering time approximation.

channels with predominantly specular boundary scattering was first reported by Choi et al., ${ }^{55}$ and studied for a narrower channel in Ref. 27 (see Section 9.b for some of these experimental results). We surmise that a classical negative magnetoresistance in the case of specular boundary scattering can result if the cyclotron radius becomes smaller than some characteristic correlation length in the distribution of impurities (and in the resulting potential landscape). Correlations between the positions of impurities and the channel boundaries, which are neglected in the scattering time approximation, will then play a role. For an example, see Fig. 12, which shows how an isolated impurity near the boundary can reverse the direction of electron motion in a zero magnetic field but not in a sufficiently strong magnetic field. In metals, where the cyclotron radius is much larger than in a $2 \mathrm{DEG}$, an electron will effectively experience a random impurity potential between subsequent boundary collisions, so the scattering can well be described in terms of an average relaxation time. The experiments in a $2 \mathrm{DEG}$ suggest that this approximation breaks down at relatively weak magnetic fields.

\section{WEAK Localization}

The temperature dependence of the Drude resistivity $\rho=m / n_{\mathrm{s}} e^{2} \tau$ is contained in that of the scattering time $\tau$, since the electron density is constant in a degenerate electron gas. As one lowers the temperature, inelastic scattering processes (such as electron-phonon scattering) are suppressed, leading to a decrease in the resistivity. The residual resistivity is due entirely 


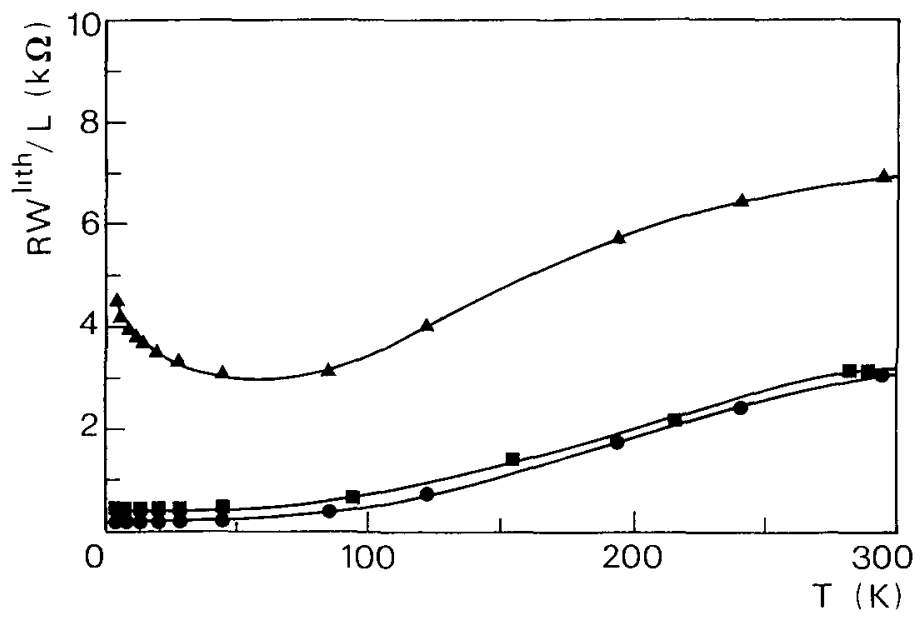

FIG 13 Temperature dependence of the resistivity of a wide 2DEG in a GaAs-AlGaAs heterostructure (circles) and of two narrow channels of lithographic width $W_{1 \text { th }}=1.5 \mu \mathrm{m}$ (squares) and $W_{\text {lith }}=05 \mu \mathrm{m}$ (triangles) The channel length $L=10 \mu \mathrm{m}$ The resistivity is estımated from the measured resistance $R$ by multiplying by $W_{\mathrm{tuth}} / L$, disregarding the difference between the conducting and lithographic width in the narrow channels Taken from $\mathrm{H}$. van Houten et al, Appl Phys Lett. 49, 1781 (1986).

to elastic scatterıng (with stationary impurities or other crystalline defects) and is temperature-independent in the semiclassical theory. Experimentally, however, one finds that below a certain temperature the resistivity of the 2DEG starts to rise again. The increase is very small in broad samples, but becomes quite pronounced in narrow channels. This is illustrated in Fig. 13, where the temperature dependencies of the resistivities of wide and narrow GaAs-AlGaAs heterostructures are compared. ${ }^{63}$

The anomalous resistivity increase is due to long-range correlations in the diffusive motion of an electron that are purely quantum mechanical. In the semiclassical theory it is assumed that a few scattering events randomize the electron velocity, so the velocity correlation function decays exponentially in time with decay time $\tau$ [see Eq. (4.19)]. As discussed in Section 4.c, this assumption leads to the Drude formula for the resistivity. It is only in recent years that one has come to appreciate that purely elastic scattering is not effective in destroying correlations in the phase of the electron wave function. Such correlations lead to quantum interference corrections to the Drude result, which can explain the anomalous increase in the resistivity at low temperatures.

A striking effect of quantum interference is to enhance the probability for backscatternng in a disordered system in the metallic regime. This effect has been interpreted as a precursor of localization in strongly disordered systems 
and has thus become known as weak localization ${ }^{115}{ }^{117}$ In Section 6 a we describe the theory for weak localization in a zero magnetic field The application of a magnetic field perpendicular to the 2DEG suppresses weak localization, ${ }^{118}$ as discussed in Section $6 \mathrm{~b}$ The resulting negative magnetoresistivity is the most convenient way to resolve experimentally the weak localization correction ${ }^{119}$ The theory for a narrow channel in the quasiballistic transport regime ${ }^{109120}$ differs in an interesting way from the theory for the diffusive regime, ${ }^{121}$ as a consequence of the flux cancellation effect ${ }^{122}$ The diffusive and quasi-ballistic regimes are the subjects of Sections $6 \mathrm{~b}$ and $6 \mathrm{c}$, respectively

\section{d Coherent Backscattering}

The theory of weak localization was developed by Anderson et al ${ }^{116}$ and Gorkov et al ${ }^{117}$ This is a diagrammatic perturbation theory that does not lend itself easily to a physical interpretation The interpretation of weak localization as coherent backscattering was put forward by Bergmann ${ }^{123}$ and by Khmel'nitski1 and Larkin, ${ }^{124}{ }^{125}$ and formed the basis of the path integral theory of Chakravarty and Schmid ${ }^{126}$ In this description, weak localization is understood by considerıng the interference of the probability amplitudes for the classical trajectories (or "Feynman paths") from one point to another, as discussed later For reviews of the alternative diagrammatic approach, we refer to Refs 127 and 128

${ }^{115} \mathrm{E}$ Abrahams, P W Anderson, D C Licciardello, and T V Ramakrishnan, Phys Rev Lett 42, 673 (1979)

${ }^{16}$ P W Anderson, E Abrahams, and T V Ramakrishnan, Phys Rev Lett 43, 718 (1979)

${ }^{117}$ L P Gorkov, A I Larkın, and D E Khmel'nıtski1, Pts'ma Zh Eksp Teor Fiz 30, 248 (1979) [JETP Lett 30, 228 (1979)]

${ }^{118}$ B L Al'tshuler, D Khmelnitski1, A I Larkın, and P A Lee, Phys Rev B 22, 5142 (1980)

${ }^{119}$ A Kawabata, $J$ Phys Soc Japan 49, 628 (1980)

${ }^{120} \mathrm{~V}$ K Dugaev and D E Khmel'nitskil, Zh Eksp Teor Flz 86, 1784 (1984) [Sov Phys JETP 59, 1038 (1984)]

${ }^{121}$ B L Al'tshuler and A G Aronov, Pis'ma Zh Eksp Teor Fiz 33, 515 (1981) [JETP Lett 33, $499(1981)]$

${ }^{122}$ P G De Gennes and M Tinkham, Phys (N Y) 1, 107 (1964), see also P G De Gennes, "Superconductivity of Metals and Alloys," Chapter 8 Benjamın, New York, 1966

${ }^{123} \mathrm{G}$ Bergmann, Phys Rep 107, 1 (1984), Phys Rev B 28, 2914 (1983)

${ }^{124}$ A I Larkin and D E Khmel'nitskı, Usp F $F_{I Z}$ Nauk 136, 536 (1982) [Sov Phys Usp 25, 185 (1982)

${ }^{125}$ D E Khmel'nitsk11, Physica 126B, 235 (1984)

${ }^{126}$ S Chakravarty and A Schmtd, Phys Rep 140, 193 (1986)

${ }^{127} \mathrm{P}$ A Lee and T V Ramakrishnan, Rev Mod Phys 57, 287 (1985)

${ }^{128}$ B L Al'tshuler, A G Aronov, D E Khmelnitsk11, and A I Larkın, in "Quantum Theory of Solids," p 130 (I M Lifshitz, ed) Advances in Science and Technology in the USSR, Physics Serres, MIR, Moskow 


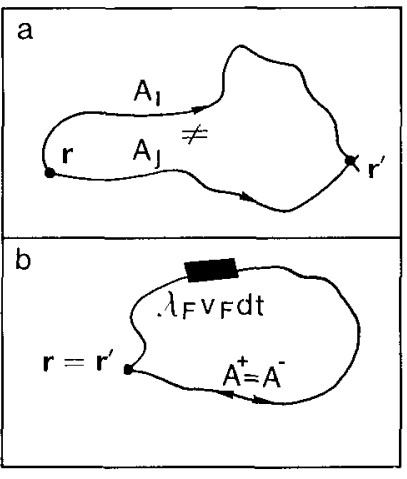

FIG 14 Mechanısm of coherent backscattering The probability amplitudes $A_{i}$ and $A_{j}$ of two trajectories from $\mathbf{r}$ to $\mathbf{r}^{\prime}$ have uncorrelated phases in general (a), but the amplitudes $A^{+}$and $A$ of two time-reversed returning trajectories are equal (b). The constructive interference of $A^{+}$and $A^{-}$increases the probability for return to the point of departure, which is the origin of the weak localization effect The volume indicated in black is the area $\lambda_{\Gamma} v_{\Gamma} d t$ covered by a flux tube in a time interval $d t$, which enters in Eq (62) for the conductivity correction

In a Feynman path description ${ }^{129}$ of diffusion, the probability $P\left(\mathbf{r}, \mathbf{r}^{\prime}, t\right)$ for motion from point $\mathbf{r}$ to point $\mathbf{r}^{\prime}$ in a time $t$ consists of the absolute value squared of the sum of probability amplitudes $A_{t}$, one for each trajectory from $\mathbf{r}$ to $\mathbf{r}^{\prime}$ of duration $t$ :

$$
P\left(\mathbf{r}, \mathbf{r}^{\prime}, t\right)=\left|\sum_{\imath} A_{t}\right|^{2}=\sum_{l}\left|A_{t}\right|^{2}+\sum_{i \neq j} A_{t} A_{l}^{*} .
$$

The restriction to classical trajectories in the sum over Feynman paths is allowed if the separation between scattering events is much larger than the wavelength (i.e., if $k_{\mathrm{F}} l \gg 1$ ). The classical diffusion probability corresponds to the first term on the right-hand side of Eq. (6.1), while the second term accounts for quantum interference. In the diffusive transport regime there is a very large number of different trajectories that contribute to the sum. One might suppose that for this reason the interference term averages out, because different trajectories have uncorrelated phases. This is correct if the beginning and end points $\mathbf{r}$ and $\mathbf{r}^{\prime}$ are different (Fig. 14a), but not if the two coincide (Fig. $14 \mathrm{~b})$. In the latter case of "backscattered" trajectories, one can group the contributions to the sum (6.1) in time-reversed pairs. Time-reversal invariance guarantees that the probability amplitudes $A^{+}$and $A^{-}$for clockwise and counterclockwise propagation around the closed loop are identical: $A^{+}=A^{-} \equiv A$. The coherent backscattering probability $\left|A^{+}+A^{-}\right|^{2}=4|A|^{2}$ is then twice the classical result. The enhanced probability for return to the point of departure reduces the diffusion constant and, hence, the conductivity. This is the essence of weak localization. As phrased by Chakravarty and Schmid, ${ }^{126}$ "1t is one of those unique cases where the superposition principle of quantum mechanics leads to observable consequences at the macroscopic level."

${ }^{129}$ R P Feynman and A R Hibbs, "Quantum Mechanıcs and Path Integrals" McGraw-Hill, New York, 1965. 
The magnitude of the weak localization correction $\delta \sigma_{\text {loc }}$ to the Drude conductivity $\sigma$ is proportional to the probability for return to the point of departure. ${ }^{126}$ Since $\delta \sigma_{10 c}$ is assumed to be a small correction, one can estimate this probability from classical diffusion. Let $C(t) d \mathbf{r}$ denote the classical probability that an electron returns after a time $t$ to within $d \mathbf{r}$ of its point of departure. The weak localization correction is given by the time integral of the return probability: ${ }^{126}$

$$
\frac{\delta \sigma_{\mathrm{loc}}}{\sigma}=-\frac{2 \hbar}{m} \int_{0}^{\infty} d t C(t) \mathrm{e}^{-t / \tau_{\phi}}
$$

The correction is negative because the conductivity is reduced by coherent backscattering. The factor $\hbar / m \propto \lambda_{F} v_{F}$ follows in the path integral formalism from the area covered by a flux tube of width $\lambda_{\mathrm{F}}$ and length $v_{\mathrm{F}} d t$ (see Fig. 14b). The factor $\exp \left(-t / \tau_{\phi}\right)$ is inserted "by hand' to account for the loss of phase coherence after a time $\tau_{\phi}$ (as a result of inelastic scattering). The return probability $C(t)$ in a $2 \mathrm{D}$ channel of width $W$ is given for times $t \gg \tau$ in the diffusive regime by

$$
\begin{aligned}
& C(t)=(4 \pi D t)^{-1}, \quad \text { if } t \ll W^{2} / D, \\
& C(t)=W^{-1}(4 \pi D t)^{-1 / 2}, \quad \text { if } t \gg W^{2} / D .
\end{aligned}
$$

The $1 / t$ decay of the return probability (6.3a) assumes unbounded diffusion in two dimensions. A crossover to a lower $1 / \sqrt{t}$ decay $(6.3 b)$ occurs when the root-mean-square displacement $(2 D t)^{1 / 2}$ exceeds the channel width, so diffusion occurs effectively in one dimension only. Because the time integral of $C(t)$ itself diverges, the weak localization correction (6.2) is determined by the behavior of the return probability on the phase coherence time $\tau_{\phi}$, which provides a long-time cutoff. One speaks of $2 \mathrm{D}$ or $1 \mathrm{D}$ weak localization, depending on whether the return probability $C\left(\tau_{\phi}\right)$ on the time scale of $\tau_{\phi}$ is determined by $2 \mathrm{D}$ diffusion (6.3a) or by $1 \mathrm{D}$ diffusion (6.3b). In terms of the phase coherence length $l_{\phi} \equiv\left(D \tau_{\phi}\right)^{1 / 2}$, the criterion for the dimensionality is that 2D weak localization occurs for $l_{\phi} \ll W$ and $1 \mathrm{D}$ weak localization for $l_{\phi} \gg W$. On short time scales $t \lesssim \tau$, the motion is ballistic rather than diffusive, and Eq. (6.3) does not apply. One expects the return probability to go to zero smoothly as one enters the ballistic regime. This short-time cutoff can be accounted for heuristically by the factor $1-\exp (-t / \tau)$, to exclude those electrons that at time $t$ have not been scattered. ${ }^{109}$ The form of the short-time cutoff becomes irrelevant for $\tau_{\phi} \gg \tau$. (See Ref. 130 for a theoretical study of weak localization in the regime of comparable $\tau_{\phi}$ and $\tau$.)

${ }^{130} \mathrm{H} \mathrm{P}$ Wittman and A Schmid, J Low Temp Phys 69, 131 (1987) 
The foregoing analysis gives the following expressions for the $2 \mathrm{D}$ and $1 \mathrm{D}$ weak localization corrections:

$$
\begin{aligned}
\delta \sigma_{\mathrm{loc}} & =-\frac{2 \hbar}{m} \sigma \int_{0}^{\infty} d t(4 \pi D t)^{-1}\left(1-e^{-t / \tau}\right) e^{-t / \tau_{\phi}} \\
& =-g_{\mathrm{s}} g_{\mathrm{v}} \frac{e^{2}}{4 \pi^{2} \hbar} \ln \left(1+\frac{\tau_{\phi}}{\tau}\right), \quad \text { if } l_{\phi} \ll W, \\
\delta \sigma_{\mathrm{loc}} & =-\frac{2 \hbar}{m} \sigma \int_{0}^{\infty} d t W^{-1}(4 \pi D t)^{-1 / 2}\left(1-e^{-t / \tau)} e^{-t / \tau_{\phi}}\right. \\
& =-g_{\mathrm{s}} g_{\mathrm{v}} \frac{e^{2}}{2 \pi \hbar} \frac{l_{\phi}}{W}\left(1-\left(1+\frac{\tau_{\phi}}{\tau}\right)^{-1 / 2}\right), \quad \text { if } l_{\phi} \gg W,
\end{aligned}
$$

where we have used the expression for the Drude conductivity $\sigma=e^{2} \rho\left(E_{F}\right) D$ with the $2 \mathrm{D}$ density of states (4.2). The ratio of the weak localization correction to the Drude conductivity $\delta \sigma_{\mathrm{loc}} / \sigma$ is of order $1 / k_{\mathrm{F}} l$ for $2 \mathrm{D}$ weak localization and of order $\left(l_{\phi} / W\right)\left(1 / k_{\mathrm{F}} l\right)$ for $1 \mathrm{D}$ weak localization. In the $2 \mathrm{D}$ case, the correction is small (cf. the values of $k_{\mathrm{F}} l$ given in Table I), but still much larger than in a typical metal. The correction is greatly enhanced in the $1 \mathrm{D}$ case $l_{\phi} \gg W$. This is evident in the experimental curves in Fig. 13, in which the resistivity increase at low temperatures is clearly visible only in the narrowest channel.

The weak localization correction to the conductance $\delta G_{\mathrm{loc}} \equiv(W / L) \delta \sigma_{\mathrm{loc}}$ is of order $\left(e^{2} / h\right)(W / L)$ in the 2D case and of order $\left(e^{2} / h\right)\left(l_{\phi} / L\right)$ in the 1D case. In the latter case, the conductance correction does not scale with the channel width $W$, contrary to what one would have classically. The conductance does scale with the reciprocal of the channel length $L$, at least for $L \gg l_{\phi}$. The factor $l_{\phi} / L$ in $\delta G_{\text {loc }}$ in the $1 \mathrm{D}$ case can be viewed as a consequence of the classical series addition of $L / l_{\phi}$ channel sections. It will then be clear that the scaling with $L$ has to break down when $L \lesssim l_{\phi}$, in which case the weak localization correction saturates at its value for $L \approx l_{\phi}$. The maximum conductance correction in a narrow channel is thus of order $e^{2} / h$, independent of the properties of the sample. This "universality" is at the origin of the phenomenon of the universal conductance fluctuations discussed in Section 7.

\section{b. Suppression of Weak Localization by a Magnetic Field}

(1) Theory. The resistance enhancement due to weak localization can be suppressed by the application of a weak magnetic field oriented perpendicular to the 2DEG. The suppression results from the fact that a magnetic field breaks time-reversal invariance. We recall that in a zero magnetic field, time-reversal invariance guarantees that trajectories that form 
a closed loop have equal probability amplitudes $A^{+}$and $A^{-}$for clockwise and counterclockwise propagation around the loop The resulting constructıve interference enhances the backscattering probability, thereby leading to the weak localization effect In a weak magnetic field, however, a phase difference $\phi$ develops between $A^{+}$and $A^{-}$, even if the curvature of the trajectories by the Lorentz force can be totally neglected This AharonovBohm phase results from the fact that the canonical momentum $\mathbf{p}=m \mathbf{v}-e \mathbf{A}$ of an electron in a magnetic field contains the vector potential A On clockwise $(+)$ and counterclockwise $(-)$ propagation around a closed loop, one thus acquires a phase difference

$$
\begin{aligned}
\phi & =\hbar^{-1} \oint_{+} \mathbf{p}^{+} \cdot d \mathbf{l}-\hbar^{-1} \oint \mathbf{p}^{-} \cdot d \mathbf{l} \\
& =\frac{2 e}{\hbar} \int(\nabla \times \mathbf{A}) \cdot d \mathbf{S}=\frac{2 e B S}{\hbar} \equiv \frac{2 S}{l_{\mathrm{m}}^{2}} \equiv 4 \pi \frac{\Phi}{\Phi_{0}}
\end{aligned}
$$

The phase difference is twice the enclosed area $S$ divided by the square of the magnetic length $l_{\mathrm{m}} \equiv(\hbar / e B)^{1 / 2}$, or, alternatively, it is $4 \pi$ times the enclosed flux $\Phi$ in units of the elementary flux quantum $\Phi_{0} \equiv h / e$

Many trajectories, with a wide distribution of loop areas, contribute to the weak localization effect In a magnetıc field the loops with a large area $S \gtrsim l_{\mathrm{m}}^{2}$ no longer contribute, since on average the counterpropagating trajectories no longer interfere constructively Since trajectories enclosing a large area necessarily take a long time to complete, the effect of a magnetic field is essentrally to introduce a long-time cutoff in the integrals of Eqs (62) and (64), which is the magnetic relaxation time $\tau_{B}$ Recall that the long-time cutoff in the absence of a magnetic field is the phase coherence time $\tau_{\phi}$ The magnetic field thus begins to have a significant effect on weak localization if $\tau_{B}$ and $\tau_{\phi}$ are comparable, which occurs at a characteristic field $B_{\mathrm{c}}$ The weak localization effect can be studied experimentally by measuring the negative magnetoresistance peak associated with its suppression by a magnetic field The significance of such experiments relies on the possibility of directly determining the phase coherence time $\tau_{\phi}$ The experimental data are most naturally analyzed in terms of the conductance The magnitude of the zerofield conductance correction $\delta G_{\text {loc }}(B=0)$ follows directly from the saturation value of the magnetoconductance, according to

$$
G\left(B \gg B_{\mathrm{c}}\right)-G(B=0)=-\delta G_{\mathrm{loc}}(B=0)
$$

Once $\delta G_{\text {loc }}(B=0)$ is known, one can deduce the phase coherence length $l_{\phi}$ from Eq (64), since $D$ and $\tau$ are easily estımated from the classical part of the conductance (which dominates at slightly elevated temperatures) The magnetoconductance contains, in addition, information on the channel width $W$, which is a parameter difficult to determine otherwise, as will become clear in the discussion of the experimental situation in subsection (2) 
The effectiveness of a magnetic field in suppressing weak localization (as contained in the functional dependence of $\tau_{B}$ on $B$, or in the expression for $B_{\mathrm{c}}$ ) is determined by the average flux enclosed by backscattered trajectories of a given duration One can distınguish different regimes, depending on the relative magnitude of the channel width $W$, the mean free path $l \equiv v_{\mathrm{F}} \tau$, the magnetic length $l_{\mathrm{m}}$, and the phase coherence length $l_{\phi} \equiv\left(D \tau_{\phi}\right)^{1 / 2}$ In Table II the expressions for $\tau_{B}$ and $B_{c}$ are summarized, as obtained by various authors 109118121131 In the following, we present a simple physical interpretation that explains these results, except for the numerical prefactors We will not discuss the effects of spin-orbit scattering ${ }^{131}$ or of superconducting fluctuations, ${ }^{132}$ since these may be neglected in the systems considered in this review In this subsection we only discuss the dirty metal regime $l \ll W$ The pure metal regime $l \gg W$, in which boundary scattering plays an important role, will be discussed in Section $6 \mathrm{c}$

If $l_{\phi} \ll W$ the two-dimensional weak localization correction to the conductivity applies, given by Eq (64a) for a zero magnetic field The typical area $S$ enclosed by a backscattered trajectory on a time scale $\tau_{B}$ is then of the order $S \sim D \tau_{B}$ (assuming diffusive motion on this time scale) The corresponding phase shift is $\phi \sim D \tau_{B} / l_{\mathrm{m}}^{2}$, in view of Eq (65) The criteria $\phi \sim 1$ and $\tau_{B} \sim \tau_{\phi}$ thus imply

$$
\tau_{B} \sim l_{\mathrm{m}}^{2} / D, \quad B_{\mathrm{c}} \sim \hbar / e D \tau_{\phi} \equiv \hbar / e l_{\phi}^{2}
$$

Table II Magnetic Relaxation Time $\tau_{B}$ ANd Characteristic Field $B_{\mathrm{c}}$ for the Suppression OF 2D AND 1D WEAK LOCALIZATION ${ }^{\text {a }}$

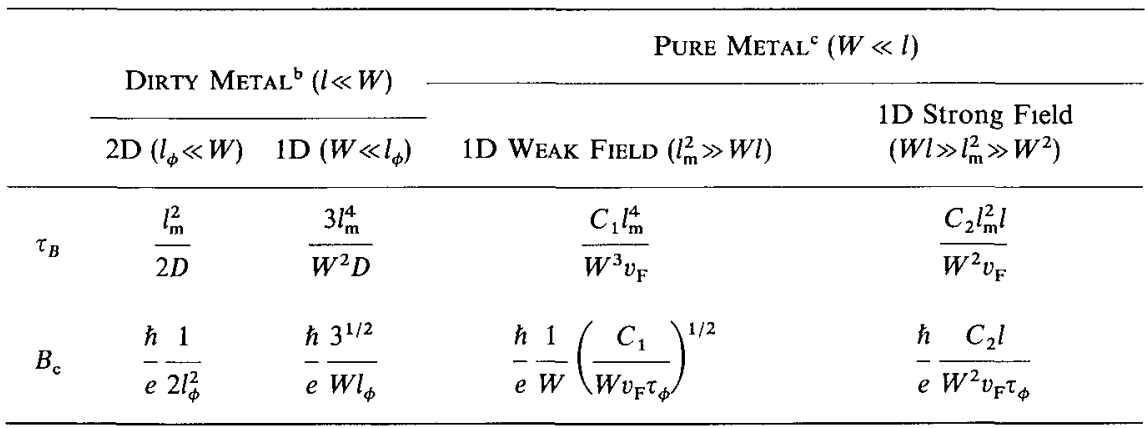

'All results assume a channel length $L \gg l_{\phi}$, a channel width $W \gg \lambda_{F}$, as well as $\tau_{\phi} \gg \tau$ bFrom Refs 118,131 , and 121 The diffusion constant $D=\frac{1}{2} v_{F} l$ If $W \ll l_{\phi}$, a transition to $2 \mathrm{D}$ weak localization occurs when $l_{\mathrm{m}} \lesssim W$

${ }^{\mathrm{c}}$ From Ref 109 The constants are given by $C_{1}=95$ and $C_{2}=24 / 5$ for specular boundary scattering $\left(C_{1}=4 \pi\right.$ and $C_{2}=3$ for a channel with diffuse boundary scattering) For pure metals, the case $l_{\mathrm{m}}<W$ is outside the diffusive transport regime for weak localization

${ }^{131}$ S Hıkamı, A I Larkın, and Y Nagaoka, Prog Theor Phys 63, 707 (1980)

${ }^{132}$ A I Larkin, Pls'ma Zh Eksp Teor Flz 31, 239 (1980) [JETP Lett 31, 219 (1980)] 
The full expression for the magnetoconductance due to weak localization 1s ${ }^{118131}$

$\delta G_{\mathrm{loc}}^{2 \mathrm{D}}(B)-\delta G_{\mathrm{loc}}^{2 \mathrm{D}}(0)=\frac{W}{L} g_{\mathrm{s}} g_{\mathrm{v}} \frac{e^{2}}{4 \pi^{2} \hbar}\left[\Psi\left(\frac{1}{2}+\frac{\tau_{B}}{2 \tau_{\phi}}\right)-\Psi\left(\frac{1}{2}+\frac{\tau_{B}}{2 \tau}\right)+\ln \left(\frac{\tau_{\phi}}{\tau}\right)\right]$,

where $\Psi(x)$ is the digamma function and $\tau_{B}=l_{\mathrm{m}}^{2} / 2 D$ The digamma function has the asymptotic approximation $\Psi(x) \approx \ln (x)-1 / x$ for large $x$, thus, in a zero magnetic field result $(64 a)$ is recovered (assuming also $\tau_{\phi} \gg \tau$ ) In the case of $2 \mathrm{D}$ weak localization the characterıstic field $B_{\mathrm{c}}$ is usually very weak For example, if $l_{\phi}=1 \mu \mathrm{m}$, then $B_{\mathbf{c}} \approx 1 \mathrm{mT}$ The suppression of the weak localization effect is complete when $\tau_{B} \lesssim \tau$, which occurs for $B \gtrsim \hbar / e D \tau \sim \hbar / e l^{2}$ These fields are still much weaker than classically strong fields for which $\omega_{\mathrm{c}} \tau \gtrsim 1$ (as can be verıfied by notıng that when $B=\hbar / e l^{2}$, one has $\left.\omega_{\mathrm{c}} \tau=1 / k_{\mathrm{F}} l \ll 1\right)$ The neglect of the curvature of electron trajectories in the theory of weak localization is thus entirely justified in the 2D case The safety margin is narrower in the 1D case, however, since the characteristic fields can become significantly enhanced

The one-dimensional case $W \ll l_{\phi}$ in a magnetic field has first been treated by Al'tshuler and Aronov ${ }^{121}$ in the dirty metal regime This refers to a narrow channel with $l \ll W$ so that the wall-to-wall motion is diffusive Since the phase coherence length exceeds the channel width, the backscattered trajectories on a time scale $\tau_{B}$ have a typical enclosed area $S \sim W\left(D \tau_{B}\right)^{1 / 2}$ (see Fig 15) Consequently, the condition $S \sim l_{\mathrm{m}}^{2}$ for a unit phase shift implies

$$
\tau_{B} \sim l_{\mathrm{m}}^{4} / D W^{2}, \quad B_{\mathrm{c}} \sim \hbar / e W l_{\phi}
$$

The difference with the $2 \mathrm{D}$ case 1 s that the enclosed flux on a given time scale is reduced, due to the lateral compression of the backscattered trajectories This leads to an enhancement by a factor $l_{\phi} / W$ of the characteristic field scale

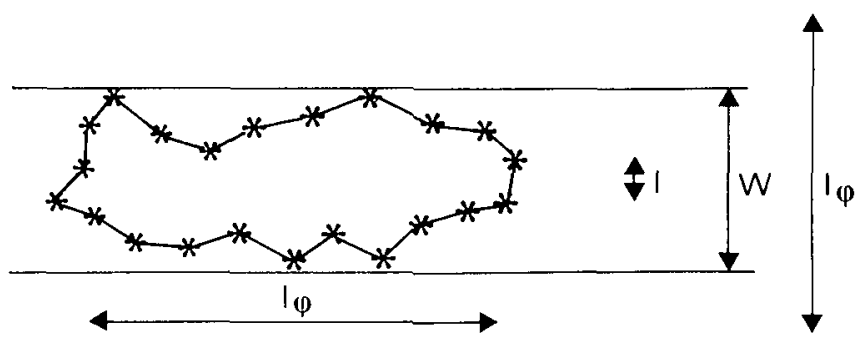

Fig 15 Typical closed electron trajectory contributing to $1 \mathrm{D}$ weak localization $\left(l_{\phi} \gg W\right)$ in the dirty metal regume $(l \ll W)$ The asterisks denote elastic scatterıng events Taken from $\mathrm{H}$ van Houten et al, Acta Electronica 28, 27 (1988) 
$B_{\mathrm{c}}$, compared with $\mathrm{Eq}(67)$ The full expression for the weak localization correction if $l_{\phi}, l_{\mathrm{m}} \gg W \gg l \mathrm{ss}^{121}$

$$
\delta G_{\mathrm{loc}}^{1 \mathrm{D}}(B)=-g_{\mathrm{s}} g_{\mathrm{v}} \frac{e^{2}}{h} \frac{1}{L}\left(\frac{1}{D \tau_{\phi}}+\frac{1}{D \tau_{B}}\right)^{-1 / 2},
$$

with $\tau_{B}=3 l_{\mathrm{m}}^{4} / W^{2} D$ For an elementary derivation of this result, see Ref 109 At $l_{\mathrm{m}} \sim W$ a crossover from $1 \mathrm{D}$ to $2 \mathrm{D}$ weak localization occurs [1 e, from Eq $(610)$ to $\mathrm{Eq} \mathrm{(68)]} \mathrm{The} \mathrm{reason} \mathrm{for} \mathrm{this} \mathrm{crossover} \mathrm{is} \mathrm{that} \mathrm{the} \mathrm{lateral}$ confinement becomes irrelevant for the weak localization when $l_{\mathrm{m}} \lesssim W$, because the trajectories of duration $\tau_{B}$ then have a typical extension $\left(D \tau_{B}\right)^{1 / 2} \lesssim W$, according to Eq (69) This crossover from 1D to 2D restricts the avalable field range that can be used to study the magnetoconductance associated with $1 \mathrm{D}$ weak localization

The magnetic relaxation time $\tau_{B}$ in the dirty metal regime is found to be inversely proportional to the diffusion constant $D$, in $2 \mathrm{D}$ as well as in $1 \mathrm{D}$ The reason for this dependence is clear faster diffusion implies that less time is needed to complete a loop of area $l_{\mathrm{m}}^{2}$ It is remarkable that in the pure metal regime such a proportionality no longer holds This is a consequence of the flux cancellation effect discussed in Section $6 \mathrm{c}$

(2) Experiments in the Dirty Metal Regime. Magnetoresıstance experiments have been widely used to study the weak localization correction to the conductivity of wide 2D electron gases in $\mathrm{Si}^{28} 30133-135$ and $\mathrm{GaAs}^{23} 136137$ Here we will discuss the experimental magnetoresistance studies of weak localization in narrow channels in Si MOSFETs ${ }^{343840138}$ and GaAsAlGaAs heterostructures ${ }^{242558}$ As an illustrative example, we reproduce in Fig 16 a set of experimental results for $\delta R / R \equiv[R(0)-R(B)] / R(0)$ obtained by Cho1 et $a^{25}$ in a wide and in a narrow GaAs-AlGaAs heterostructure The quantity $\delta R$ is positive, so the resistance decreases on applying a magnetic field The 2D results are similar to those obtained earlier by Paalanen et al ${ }^{137}$ The qualitative difference in field scale for the suppression of 2D (top) and 1D (bottom) weak localization is nicely illustrated by the data in Fig 16 The magnetoresistance peak is narrower in the $2 \mathrm{D}$ case, consistent with the enhancement in $1 \mathrm{D}$ of the characteristic field $B_{\mathrm{c}}$ for the suppression of weak localization, which we discussed in Section $6 \mathrm{~b}(1)$ The solid curves in

\footnotetext{
${ }^{133}$ Y Kawaguchı and S Kawajı, $J$ Phys Soc Jpn 48, 699 (1980)

${ }^{134}$ R G Wheeler, Phys Rev B 24, 4645 (1981)

${ }^{135}$ M J Uren, R A Davis, M Kaveh, and M Pepper, J Phys C 14, L395 (1981)

${ }^{136} \mathrm{D}$ A Poole, M Pepper, and R W Glew, $J$ Phys C 14, L995 (1981)

${ }^{137}$ M A Paalanen, D C Tsul, and J C M Hwang, Phys Rev Lett 51, 2226 (1983)

${ }^{138}$ D M Pooke R Mottdhedeh, M Pepper and A Grundlach, Suff Scl 19659 (1988), D M

Pooke N Paquın M Pepper and A Grundlach J Phys Condens Matter 13289 (1989)
} 


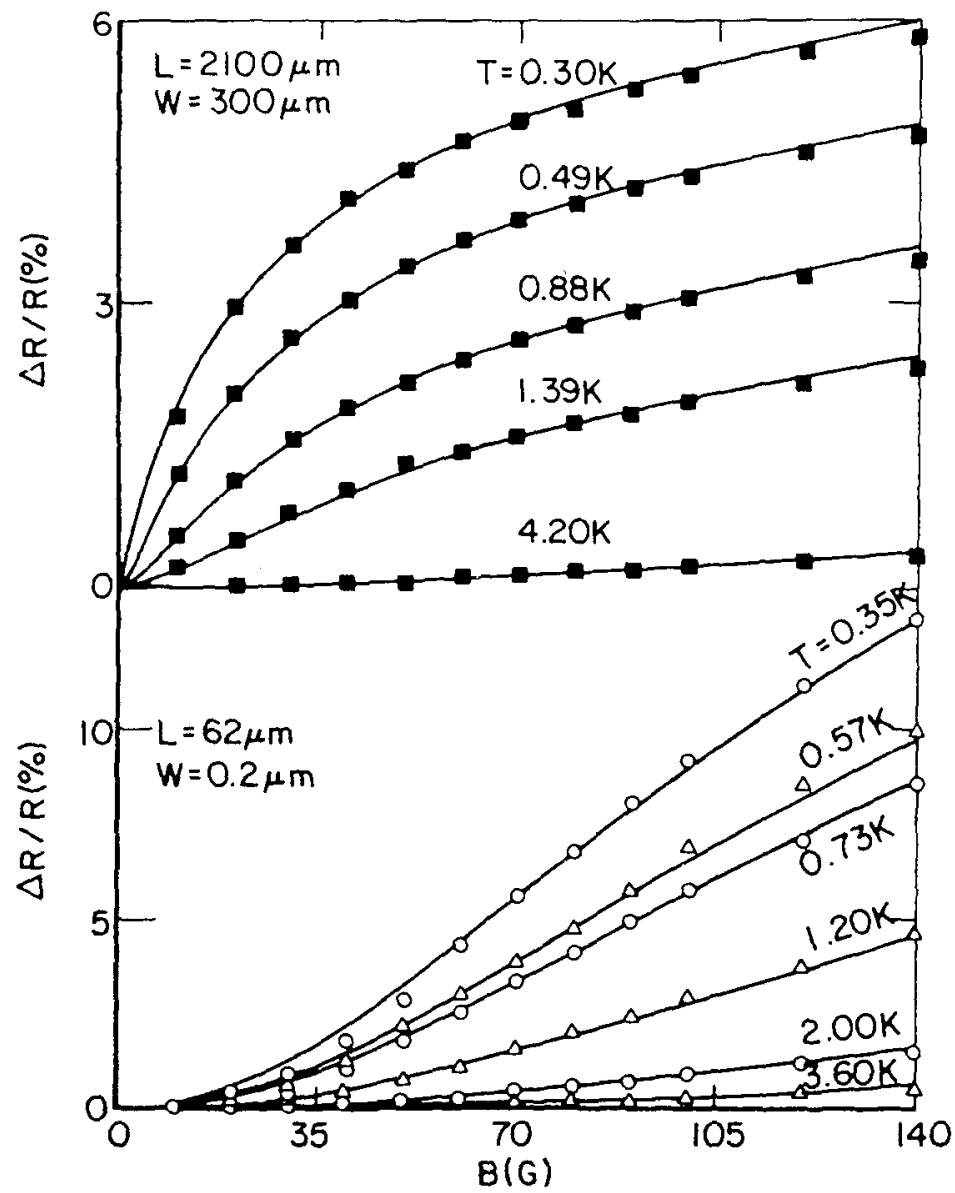

FIG. 16. A comparison between the magnetoresistance $\Delta R / R \equiv[R(0)-R(B)] / R(0)$ due to $2 D$ weak localization in a wide channel (upper panel) and due to $1 D$ weak localization in a narrow channel (lower panel), at various temperatures. The solid curves are fits based on Eqs. (6.8) and (6.10). Taken from K. K. Choi et al., Phys. Rev. B 36, 7751 (1987).

Fig. 16 were obtained from the 2D theoretical expression (6.8) and the 1D dirty metal result (6.10), treating $W$ and $l_{\phi}$ as adjustable parameters. A noteworthy finding of Choi et $a l^{25}$ is that the effective channel width $W$ is considerably reduced below the lithographic width $W_{1 \text { ith }}$ in narrow channels defined by a deep-etched mesa (as in Fig. 4a). Differences $W-W_{1 \text { ith }}$ of about $0.8 \mu \mathrm{m}$ were found. ${ }^{25}$ Significantly smaller differences are obtained ${ }^{27,63}$ if a shallow-etched mesa is used for the lateral confinement, as in Fig. 4c. A splitgate device (as in Fig. 4b) of variable width has been used by Zheng et al. ${ }^{24}$ to 
study weak localization in GaAs-AlGaAs heterostructure channels. Magnetoresistance experiments in a very narrow split-gate device (fabricated using electron beam lithography) were reported by Thornton et al. ${ }^{58}$ and analyzed in terms of the dirty metal theory. Unfortunately, in their experiment the mean free path of $450 \mathrm{~nm}$ exceeded the width inferred from a fit to Eq. (6.10) by an order of magnitude, so an analysis in terms of the pure metal theory would have been required.

Early magnetoresistance experiments on narrow Si accumulation layers were performed by Dean and Pepper, ${ }^{34}$ in which they observed evidence for a crossover from the $2 \mathrm{D}$ to the $1 \mathrm{D}$ weak localization regime. A comparison of weak localization in wide and narrow $\mathrm{Si}$ inversion layers was reported by Wheeler $e t$ al. ${ }^{38}$ The conducting width of the narrow channel was taken to be equal to the lithographic width of the gate (about $400 \mathrm{~nm}$ ), while the mean free path was estimated to be about $100 \mathrm{~nm}$. This experiment on a lowmobility Si channel thus meets the requirement $l \ll W$ for the dirty metal regime. The $1 \mathrm{D}$ weak localization condition $l_{\phi} \gg W$ was only marginally satisfied, however. Licini et al. ${ }^{40}$ reported a negative magnetoresistance peak in $270-\mathrm{nm}$-wide $\mathrm{Si}$ inversion layers, which was well described by the $2 \mathrm{D}$ theory at a temperature of $2.2 \mathrm{~K}$, where $l_{\phi}=120 \mathrm{~nm}$. Deviations from the $2 \mathrm{D}$ form were found at lower temperatures, but the $1 \mathrm{D}$ regime was never fully entered. A more recent study of $1 \mathrm{D}$ weak localization in a narrow $\mathrm{Si}$ accumulation layer has been performed by Pooke $e t$ al. ${ }^{138}$ at low temperatures, and the margins are somewhat larger in their case.

We note a difficulty inherent to experiments on $1 \mathrm{D}$ weak localization in semiconductor channels in the dirty metal regime. For $1 \mathrm{D}$ weak localization it is required that the phase coherence length $l_{\phi}$ is much larger than the channel width. If the mean free path is short, then the experiment is in the dirty metal regime $l \ll W$, but the localization will be only marginally onedimensional since the phase coherence length $l_{\phi} \equiv\left(D \tau_{\phi}\right)^{1 / 2}=\left(v_{F} l \tau_{\phi} / 2\right)^{1 / 2}$ will be short as well (except for the lowest experimental temperatures). If the mean free path is long, then the $1 \mathrm{D}$ criterion $l_{\phi} \gg W$ is easily satisfied, but the requirement $l \ll W$ will now be hard to meet so that the experiment will tend to be in the pure metal regime. A quantitative comparison with the theory (which would allow a reliable determination of $l_{\phi}$ ) is hampered because the asymptotic regimes studied theoretically are not accessible experimentally and because the channel width is not known a priori. Nanostructures are thus not the best candidates for a quantitative study of the phase coherence length, which is better studied in 2D systems. An altogether different complication is that quantum corrections to the conductivity in semiconductor nanostructures can be remarkably large (up to $100 \%$ at sufficiently low temperatures ${ }^{27,34}$ ), which puts them beyond the range of validity of the perturbation theory. 


\section{$\oplus \mathrm{B}$}

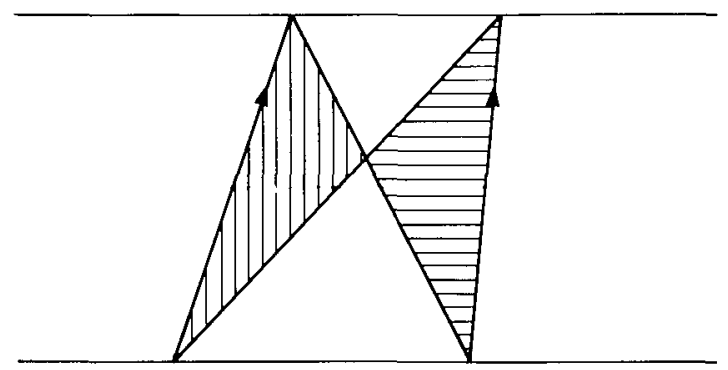

FIG 17 Illustration of the flux cancellation effect for a closed trajectory of one electron in a narrow channel with diffuse boundary scattering The trajectory is composed of two loops of equal area but opposite orientation, so 1 t encloses zero flux Taken from C W J Beenakker and H van Houten, Phys Rev B 38, 3232 (1988)

\section{c Boundary Scattering and Flux Cancellation}

(1) Theory. In the previous subsection we noticed that the pure metal regime, where $l \gg W$, is characteristic for $1 \mathrm{D}$ weak localization in semiconductor nanostructures This regime was first theoretically considered by Dugaev and Khmel'nitski1, ${ }^{120}$ for the geometry of a thin metal film in a parallel magnetıc field and for diffuse boundary scatterıng The geometry of a narrow 2DEG channel in a perpendicular magnetic field, with either diffuse or specular boundary scattering, was treated by the present authors ${ }^{109}$ Note that the nature of the boundary scattering did not play a role in the dirty metal regime of Section $6 \mathrm{~b}$, since there the channel walls only serve to impose a geometrical restriction on the lateral diffusion ${ }^{121}$ The flux cancellation effect is characteristic of the pure metal regime, where the electrons move ballistically from one wall to the other This effect (which also plays a role in the superconductivity of thin films in a parallel magnetic field ${ }^{122}$ ) leads to a further enhancement of the characteristic field scale $B_{\mathrm{c}}$ Flux cancellation results from the fact that typically backscattered trajectories for $l \gg W$ selfintersect (cf Fig 17) and are thus composed of smaller loops that are traversed in opposite directions Zero net flux is enclosed by closed trajectories involving only wall collisions (as indicated by the shaded areas in Fig 17, which are equal but of opposite orientation), so impurity collisions are required for phase relaxation in a magnetic field This is in contrast to the dirty metal regime considered before, where impurity scatterıng hinders phase relaxation by reducing the diffusion constant The resulting nonmonotonous dependence of phase relaxation on impurity scattering in the dirty and pure metal regimes is illustrated in Fig 18, where the calculated ${ }^{109}$ 


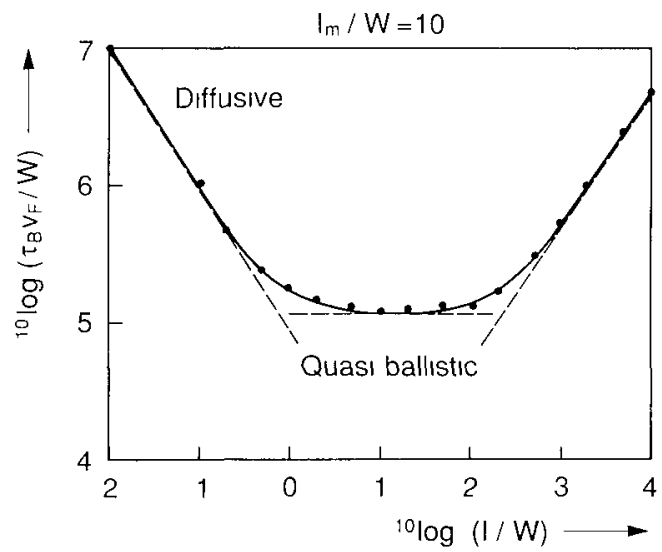

Fig 18 Phase relaxation time $\tau_{\mathrm{B}}$ in a channel with specular boundary scattering, as a function of the elastic mean free path $l$ The plot has been obtained by a numerical simulation of the phase relaxation process for a magnetic field such that $l_{\mathrm{m}}=10 \mathrm{~W}$ The dashed lines are analytic formulas valid in the three asymptotic regimes (see Table II) Taken from C W J Beenakker and H van Houten Phys Rev B 38, 3232 (1988)

magnetic relaxation time $\tau_{B}$ is plotted as a function of $l / W$ for a fixed ratio $l_{\mathrm{m}} / W$

Before continuing our discussion of the flux cancellation effect, we give a more precise definition of the phase relaxation time $\tau_{B}$ The effect of a magnetic field on weak localization is accounted for formally by insertıng the term

$$
\left\langle e^{1 \phi(t)} \mid \mathbf{r}(t)=\mathbf{r}(0)\right\rangle=e^{-t / \tau_{B}}, \quad W \ll l_{\mathrm{m}}, l_{\phi},
$$

in the integrand of $\mathrm{Eq}(62)$ The term (6 11) is the conditional average over all closed trajectories having duration $t$ of the phase factor $\mathrm{e}^{t \phi(t)}$, with $\phi$ the phase difference defined in Eq (6 5) It can be shown ${ }^{109}$ that in the case of 1D weak localization (and for $l_{\mathrm{m}} \gg W$ ), this term is given by an exponential decay factor $\exp \left(-t / \tau_{B}\right)$, which defines the magnetıc relaxation time $\tau_{B}$ In this regime the weak localization correction to the conductivity in the presence of a magnetic field is then simply given by Eq (64b), after the substitution

$$
\tau_{\phi}{ }^{1} \rightarrow \tau_{\phi}{ }^{1}+\tau_{B}^{-1}
$$

Explicitly, one obtains

$$
\delta G_{\mathrm{loc}}(B)=-g_{\mathrm{s}} g_{\mathrm{v}} \frac{e^{2}}{h} \frac{1}{L}\left(\left[\frac{1}{D \tau_{\phi}}+\frac{1}{D \tau_{B}}\right]^{1 / 2}-\left[\frac{1}{D \tau_{\phi}}+\frac{1}{D \tau_{B}}+\frac{1}{D \tau}\right]^{-1 / 2}\right)
$$


One can see from Fig. 18 and Table II that in the pure metal regime $l \gg W$, a weak and strong field regime can be distinguished, depending on the ratio $W l / l_{\mathrm{m}}^{2}$. This ratio corresponds to the maximum phase change on a closed trajectory of linear extension $l$ (measured along the channel). In the weak field regime $\left(W l / l_{\mathrm{m}}^{2} \ll 1\right)$ many impurity collisions are required before a closed electron loop encloses sufficient flux for complete phase relaxation. In this regime a further increase of the mean free path does not decrease the phase relaxation time (in contrast to the dirty metal regime), because as a consequence of the flux cancellation effect, faster diffusion along the channel does not lead to a larger enclosed flux. On comparing the result in Table II for $B_{\mathrm{c}}$ in the weak field regime with that for the dirty metal regime, one sees an enhancement of the characteristic field by a factor $(l / W)^{1 / 2}$. The strong field regime is reached if $W l / l_{\mathrm{m}}^{2} \gg 1$, while still $l_{\mathrm{m}} \gg W$. Under these conditions, a single impurity collision can lead to a closed trajectory that encloses sufficient flux for phase relaxation. The phase relaxation rate $1 / \tau_{B}$ is now proportional to the impurity scattering rate $1 / \tau$ and, thus, to $1 / l$. The relaxation time $\tau_{B}$ accordingly increases linearly with $l$ in this regime (see Fig. 18). For comparison with experiments in the pure metal regime, an analytic formula that interpolates between the weak and strong field regimes is useful. The following formula agrees well with numerical calculations: ${ }^{109}$

$$
\tau_{B}=\tau_{B}^{\mathrm{weak}}+\tau_{B}^{\text {strong }}
$$

Here $\tau_{B}^{\text {weak }}$ and $\tau_{B}^{\text {strong }}$ are the expressions for $\tau_{B}$ in the asymptotic weak and strong field regimes, as given in Table II.

So far, we have assumed that the transport is diffusive on time scales corresponding to $\tau_{\phi}$. This will be a good approximation only if $\tau_{\phi} \gg \tau$. Coherent diffusion breaks down if $\tau_{\phi}$ and $\tau$ are of comparable magnitude (as may be the case in high-mobility channels). The modification of weak localization as one enters the ballistic transport regime has been investigated by Wittmann and Schmid. ${ }^{130}$ It would be of interest to see to what extent the ad hoc short-time cutoff introduced in our Eq. (6.4), which is responsible for the second bracketed term in Eq. (6.13), is satisfactory.

(2) Experiments in the Pure Metal Regime. Because of the high mobility required, the pure metal regime has been explored using GaAs-AlGaAs heterostructures only. The first experiments on weak localization in the pure metal regime were done by Thornton et al., ${ }^{58}$ in a narrow split-gate device, although the data were analyzed in terms of the theory for the dirty metal regime. An experimental study specifically aimed at weak localization in the pure metal regime was reported in Refs. 26 and 27. In a narrow channel defined by the shallow-mesa etch technique of Fig. 4c (with a conducting width estimated at $0.12 \mu \mathrm{m}$ ), a pronounced negative magnetoresistance effect 


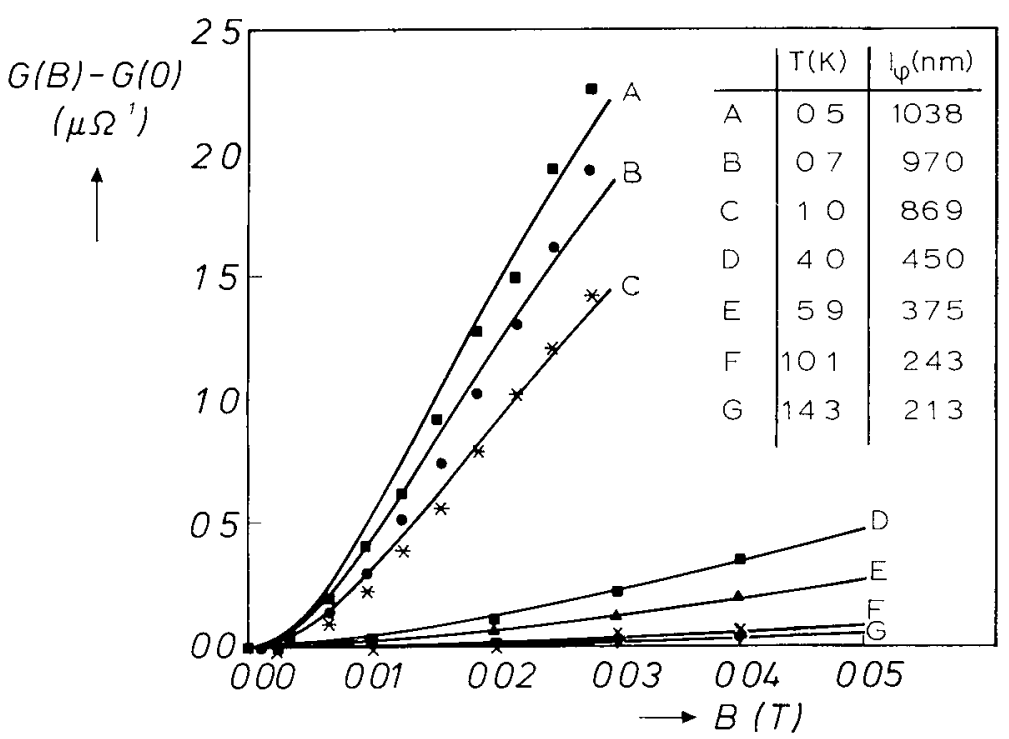

FIG 19 Magnetoconductance due to ID weak localization in the pure metal regime ( $W=120 \mathrm{~nm}, L=350 \mathrm{~nm}$ ) The solid curves are one-parameter fits to $\mathrm{Eq}$ (6 13) Only the field range $l_{\mathbf{m}}>W$ is shown in accordance with the condition of coherent diffusion imposed by the theory The phase coherence length $l_{\phi}$ obtained from the data at various temperatures is tabulated in the insel Taken from H van Houten $c t$ al Suif Sct 196, 144 (1988)

was found, similar to that observed by Thornton et al ${ }^{58}$ A good agreement of the experimental results with the theory ${ }^{109}$ for weak localization in the pure metal regime was obtained (see Fig 19), assuming specular boundary scatterıng (diffuse boundary scattering could not describe the data) The width deduced from the analysis was consistent with independent estimates from other magnetoresistance effects Further measurements in this regime were reported by Chang et al ${ }^{70139}$ and, more recently, by Huramoto et al ${ }^{81}$ These experıments were also well described by the theory of Ref 109

\section{Conductance Fluctuations}

Classically, sample-to-sample fluctuations in the conductance are negligible in the diffusive (or quasi-ballistic) transport regime In a narrowchannel geometry, for example, the root-mean-square $\delta G_{\text {clas }}$ of the classical fluctuations in the conductance is smaller than the average conductance $\langle G\rangle$ by a factor $(l / L)^{1 / 2}$, under the assumption that the channel can be subdivided into $L / l \gg 1$ independently fluctuating segments As we have discussed in the ${ }^{139}$ A M Chang, G Timp, R E Howard, R E Behrınger, P M Mankıewich, J E Cunningham,

T Y Chang, and B Chelluri, Superlattices and Mtcrostructures, 4, 515 (1988) 
previous section, however, quantum mechanical correlations persist over a phase coherence length $l_{\phi}$ that can be much larger than the elastic mean free path $l$ Quantum interference effects lead to significant sample-to-sample fluctuations in the conductance if the size of the sample is not very much larger than $l_{\phi}$ The Al'tshuler-Lee-Stone theory of Universal Conductance Fluctuations ${ }^{140141}$ finds that $\delta G \approx e^{2} / h$ at $T=0$, when phase coherence is maintained over the entire sample Since $\langle G\rangle \propto L^{1}$, it follows that $\delta G /\langle G\rangle \propto L$ increases with increasing channel length, that is, there is a total absence of self-averaging

Experımentally, the large sample-to-sample conductance fluctuations predicted theoretically are difficult to study in a direct way, because of problems in the preparation of samples that differ in impurity configuration only (to allow an ensemble average) The most convenient way to study the effect is via the fluctuations in the conductance of a single sample as a function of magnetic field, because a small change in field has a sımilar effect on the interference pattern as a change in impurity configuration Sections $7 \mathrm{c}$ and $7 \mathrm{~d}$ deal with theoretical and experimental studies of magnetoconductance fluctuations in narrow 2DEG channels, mainly in the quasi-ballistic regime characteristic for semiconductor nanostructures In Sections $7 \mathrm{a}$ and $7 \mathrm{~b}$ we discuss the surprising universality of the conductance fluctuations at zero temperature and the finite-temperature modifications

\section{a Zero-Temperature Conductance Fluctuations}

The most surprising feature of the conductance fluctuations is that their magnitude at zero temperature is of order $e^{2} / h$, regardless of the size of the sample and the degree of disorder, ${ }^{140141}$ provided at least that $L \gg l$, so that transport through the sample is diffusive (or possibly quasi-ballistic) Lee and Stone $^{141}$ coined the term Universal Conductance Fluctuations (UCF) for this effect In this subsection we give a simplified explanation of this universality due to Lee ${ }^{142}$

Consider first the classical Drude conductance (4 8) for a single spin direction (and a single valley)

$$
G=\frac{W}{L} \frac{e^{2}}{h} \frac{k_{\mathrm{F}} l}{2}=\frac{e^{2}}{h} \frac{\pi l}{2 L} N, \quad N \equiv \frac{k_{\mathrm{F}} W}{\pi}
$$

The number $N$ equals the number of transverse modes, or one-dimensional subbands, that are occupied at the Fermı energy in a conductor of width $W$ We have written the conductance in this way to make contact with the

\footnotetext{
${ }^{140} \mathrm{~B}$ L Al'tshuler, Pts'ma Zh Eksp Teor Ftz 41, 530 (1985) [JETP Lett 41, 648 (1985)]

${ }^{141} \mathrm{P}$ A Lee and A D Stone, Phys Rev Lett 55, 1622 (1985)

${ }^{142}$ P A Lee, Physica 140A, 169 (1986)
} 


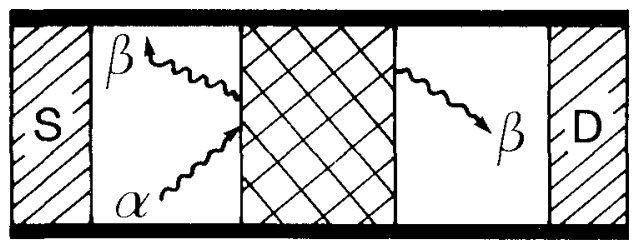

FIG 20 Idealized conductor connecting source (S) and drain (D) reservoirs and containing a disordered region (crosshatched) The incoming quantum channels (or transverse waveguide modes) are labeled by $\alpha$, the transmitted and backscattered channels by $\beta$

Landauer approach ${ }^{4}$ to conduction, which relates the conductance to the transmission probabilities of modes at the Fermı energy (A detalled discussion of this approach is given the context of quantum ballistic transport in Section 12 b) The picture to have in mind is shown in Fig 20 Current is passed from a source reservoir $\mathrm{S}$ to a drain reservoir $\mathrm{D}$, through a disordered region (hatched) in which only elastic scattering takes place The two reservoirs are in thermal equilibrium and are assumed to be fully effective in randomizing the phase via inelastic scattering, so there is no phase coherence between the $N$ modes incident on the disordered region The modes in this context are called quantum channels If $L \gg l$, each channel has on average the same transmission probability, given by $\pi l / 2 L$ according to Eqs $(421)$ and (7 1) We are interested in the fluctuations around this average The resulting fluctuations in $G$ then follow from the multichannel Landauer formula ${ }^{1143144}$

$$
G=\frac{e^{2}}{h} \sum_{\alpha \beta-1}^{N}\left|t_{\alpha \beta}\right|^{2},
$$

where $t_{\beta \alpha}$ denotes the quantum mechanical transmission probability amplitude from the incident channel $\alpha$ to the outgoing channel $\beta$ (cf Fig 20) The ensemble averaged transmission probability $\left\langle\left|t_{\alpha \beta}\right|^{2}\right\rangle$ does not depend on $\alpha$ or $\beta$, so the correspondence between Eqs (7 1) and (72) requires

$$
\left\langle\left|t_{\alpha \beta}\right|^{2}\right\rangle=\pi l / 2 N L
$$

The magnitude of the conductance fluctuations is characterized by its variance $\operatorname{Var}(G) \equiv\left\langle(G-\langle G\rangle)^{2}\right\rangle$ As discussed by Lee, a difficulty arıses in a direct evaluation of $\operatorname{Var}(G)$ from Eq (72), because the correlation in the transmission probabilities $\left|t_{\alpha \beta}\right|^{2}$ for different pairs of incident and outgoing channels $\alpha, \beta$ may not be neglected ${ }^{142}$ The reason is presumably that transmission through the disordered region involves a large number of impurity collisions, so a sequence of scattering events will in general be

\footnotetext{
${ }^{143}$ D S Fisher and P A Lee, Phys Rev B 23, 6851 (1981)
}

${ }^{144}$ A D Stone, in Ref 14 
shared by different channels. On the same grounds, it is reasonable to assume that the reflection probabilities $\left|r_{\alpha \beta}\right|^{2}$ for different pairs $\alpha \beta$ of incident and reflected channels are uncorrelated, since the reflection back into the source reservoir would seem to be dominated by only a few scattering events. ${ }^{142}$ (The formal diagrammatic analysis of Refs. 140 and 141 is required here for a convincing argument.) The reflection and transmission probabilities are related by current conservation

$$
\sum_{\alpha, \beta=1}^{N}\left|t_{\alpha \beta}\right|^{2}=N-\sum_{\alpha, \beta=1}^{N}\left|r_{\alpha \beta}\right|^{2},
$$

so the variance of the conductance equals

$$
\operatorname{Var}(G)=\left(\frac{e^{2}}{h}\right)^{2} \operatorname{Var}\left(\sum\left|r_{\alpha \beta}\right|^{2}\right)=\left(\frac{e^{2}}{h}\right)^{2} N^{2} \operatorname{Var}\left(\left|r_{\alpha \beta}\right|^{2}\right)
$$

assuming uncorrelated reflection probabilities. A large number $M$ of scattering sequences through the disordered region contributes with amplitude $A(l)(i=1,2, \ldots, M)$ to the reflection probability amplitude $r_{\alpha \beta}$. (The different scattering sequences can be seen as independent Feynman paths in a path integral formulation of the problem. ${ }^{142}$ ) To calculate $\operatorname{Var}\left(\left|r_{\alpha \beta}\right|^{2}\right)=\left\langle\left|r_{\alpha \beta}\right|^{4}\right\rangle-\left\langle\left|r_{\alpha \beta}\right|^{2}\right\rangle^{2}$, one may then write (neglecting correlations in $A(i)$ for different $i$ )

$$
\begin{aligned}
\left\langle\left|r_{\alpha \beta}\right|^{4}\right\rangle & =\sum_{l, j, k, l=1}^{M}\left\langle A^{*}(i) A(j) A^{*}(k) A(l)\right\rangle \\
& =\sum_{l, j, k, l=1}^{M}\left\{\left\langle|A(i)|^{2}\right\rangle\left\langle|A(k)|^{2}\right\rangle \delta_{l} \delta_{k l}+\left\langle|A(i)|^{2}\right\rangle\left\langle|A(j)|^{2}\right\rangle \delta_{l l} \delta_{J k}\right\} \\
& =2\left\langle\left|r_{\alpha \beta}\right|^{2}\right\rangle^{2},
\end{aligned}
$$

where we nave neglected terms smaller by a factor $1 / M$ (assuming $M \gg 1$ ). One thus finds that the variance of the reflection probability is equal to the square of its average:

$$
\operatorname{Var}\left(\left|r_{\alpha \beta}\right|^{2}\right)=\left\langle\left|r_{\alpha \beta}\right|^{2}\right\rangle^{2} \text {. }
$$

The average reflection probability $\left\langle\left|r_{\alpha \beta}\right|^{2}\right\rangle$ does not depend on $\alpha$ and $\beta$. Thus, from Eqs. (7.3) and (7.4) it follows that

$$
\left\langle\left|r_{\alpha \beta}\right|^{2}\right\rangle=N^{-1}(1-\operatorname{order}(l / L)) \text {. }
$$

Combining Eqs. (7.5), (7.7), and (7.8), one obtains the result that the zerotemperature conductance has a variance $\left(e^{2} / h\right)^{2}$, independent of $l$ or $L$ (in the diffusive limit $l \ll L$ ). We have discussed this argument of Lee in some detail, because no other simple argument known to us gives physical insight in this remarkable result. 
The numerical prefactors follow from the diagrammatic analysis ${ }^{140141145146}$ The result of Lee and Stone ${ }^{141}$ for the root-mean-square magnitude of the conductance fluctuations at $T=0$ can be written in the form

$$
\delta G \equiv[\operatorname{Var}(G)]^{1 / 2}=\frac{g_{\mathrm{s}} g_{\mathrm{v}}}{2} \beta^{-1 / 2} C \frac{e^{2}}{h}
$$

Here $C$ is a constant that depends on the shape of the sample Typically, $C$ is of order unity, for example, $C \approx 073$ in a narrow channel with $L \gg W$ (However, in the opposite limit $W \gg L$ of a wide and short channel, $C$ is of order $(W / L)^{1 / 2}$ ) The parameter $\beta=1$ in a zero magnetic field when timereversal symmetry holds, $\beta=2$ when time-reversal symmetry is broken by a magnetic field The factor $g_{\mathrm{s}} g_{\mathrm{v}}$ assumes complete spin and valley degeneracy If the magnetic field is sufficiently strong that the two spin directions give statistically independent contributions to the conductance, then the variances add so that the factor $g_{\mathrm{s}}$ in $\delta G$ is to be replaced by a factor $g_{\mathrm{s}}^{1 / 2}$ We will return to this point in Section $7 \mathrm{~d}$

\section{b Nonzero Temperatures}

At nonzero temperatures, the magnitude of the conductance fluctuations is reduced below $\delta G \approx e^{2} / h$ One reason is the effect of a finite phase coherence length $l_{\phi} \equiv\left(D \tau_{\phi}\right)^{1 / 2}$, another is the effect of thermal averaging, as expressed by the thermal length $l_{\mathrm{T}} \equiv\left(\hbar D / k_{B} T\right)^{1 / 2}$ The effect of a finite temperature, contained in $l_{\phi}$ and $l_{\mathrm{T}}$, is to partally restore self-averaging, albeit that the suppression of the fluctuation with sample size is much weaker than would be the case classically The theory has been presented clearly and in detail by Lee, Stone, and Fukuyama ${ }^{145}$ We limit the present discussion to the 1D regıme $W \ll l_{\phi} \ll L$, characteristic for narrow 2DEG channels

The effects of thermal averaging may be neglected if $l_{\phi} \ll l_{\mathrm{T}}$ (see below) The channel may then be thought to be subdivided in uncorrelated segments of length $l_{\phi}$ The conductance fluctuation of each segment individually will be of order $e^{2} / h$, as it is at zero temperature The root-mean-square conductance fluctuation of the entire channel is easily estımated The segments are in series, so their resistances add according to Ohm's law We denote the resistance of a channel segment of length $l_{\phi}$ by $R_{1}$ The variance of $R_{1}$ is $\operatorname{Var}\left(R_{1}\right) \approx\left\langle R_{1}\right\rangle^{4} \operatorname{Var}\left(R_{1}{ }^{1}\right) \approx\left\langle R_{1}\right\rangle^{4}\left(e^{2} / h\right)^{2}$ The average resistance of the whole channel $\langle R\rangle=\left(L / l_{\phi}\right)\left\langle R_{1}\right\rangle$ increases linearly with the number $L / l_{\phi}$ of uncorrelated channel segments, just as its variance $\operatorname{Var}(R)=$

\footnotetext{
${ }^{145}$ P A Lee, A D Stone, and H Fukuyama, Phys Rev B 35, 1039 (1987)

${ }^{146} \mathrm{~B}$ L Al'tshuler and D E Khmel'nitsku, Pis'ma Zh Eksp Teor Fiz 42, 291 (1985) [JETP Lett 42359 (1985)]
} 
$\left(L / l_{\phi}\right) \operatorname{Var}\left(R_{1}\right) \approx\left(L / l_{\phi}\right)\left\langle R_{1}\right\rangle^{4}\left(e^{2} / h\right)^{2}$ (The root-mean-square resistance fluctuation thus grows as $\left(L / l_{\phi}\right)^{1 / 2}$, the square root of the number of channel segments in series ) Expressed in terms of a conductance, one thus has $\operatorname{Var}(G) \approx\langle R\rangle{ }^{4} \operatorname{Var}(R) \approx\left(l_{\phi} / L\right)^{3}\left(e^{2} / h\right)^{2}$, or

$$
\delta G=\text { constant } \times \frac{e^{2}}{h}\left(\frac{l_{\phi}}{L}\right)^{3 / 2}, \quad \text { if } l_{\phi} \ll l_{\mathrm{T}}
$$

The constant prefactor is given in Table III

We now turn to the second effect of the finite temperature, which is the smearing of the fluctuations by the energy average within an interval of order $k_{\mathrm{B}} T$ around the Fermi energy $E_{\mathrm{F}}$ Note that we did not have to consider this thermal averaging in the context of the weak localization effect, since that is a systematic, rather than a fluctuatıng, property of the sample Two interferıng Feynman paths, traversed with an energy difference $\delta E$, have to be considered as uncorrelated after a time $t_{1}$, ff the acquired phase difference $t_{1} \delta E / \hbar$ is of order unity In this time the electrons diffuse a distance $L_{1}=\left(D t_{1}\right)^{1 / 2} \sim(\hbar D / \delta E)^{1 / 2}$ One can now define a correlation energy $E_{\mathrm{c}}\left(L_{1}\right)$, as the energy difference for which the phase difference following diffusion over a distance $L_{1}$ is unity

$$
E_{\mathrm{c}}\left(L_{1}\right) \equiv \hbar D / L_{1}^{2}
$$

The thermal length $l_{\mathrm{T}}$ is defined such that $E_{\mathrm{c}}\left(l_{\mathrm{T}}\right) \equiv k_{\mathrm{B}} T$, which implies

$$
l_{\mathrm{T}} \equiv\left(\hbar D / k_{B} T\right)^{1 / 2}
$$

Table III Asymptotic Expressions for the RoOt-Mean-SQuare Conductance Fluctuations in a Narrow ChanNel a

\begin{tabular}{cccc} 
& $\begin{array}{c}T=0 \\
l_{\mathrm{T}}, l_{\phi} \gg L\end{array}$ & $l_{\phi} \ll L, l_{\mathrm{T}}$ & $l_{\mathrm{T}} \ll l_{\phi} \ll L$ \\
\hline$\delta G \times \frac{2}{g_{\mathrm{s}} g_{\mathrm{v}}} \beta^{1 / 2}$ & $C \frac{e^{2}}{h}$ & $C \frac{e^{2}}{h}\left(\frac{l_{\phi}}{L}\right)^{3 / 2}$ & $C \frac{e^{2}}{h} \frac{l_{\mathrm{T}} l_{\phi}^{1 / 2}}{L^{3 / 2}}$ \\
$C$ & 073 & $\sqrt{12}$ & $\left(\frac{8 \pi}{3}\right)^{1 / 2}$ \\
\hline
\end{tabular}

${ }^{\mathrm{a}}$ The results assume a narrow channel $(W \ll L)$, with a $2 \mathrm{D}$ density of states $\left(W \gg \lambda_{\mathrm{F}}\right.$ ), which is in the 1D limit for the conductance fluctuat1ons $\left(W \ll l_{\phi}\right)$ The expressions for $\delta G$ are from Refs $140,141,145$, and 146 The numerical prefactor $C$ for $T=0$ is from $\operatorname{Ref} 141$, for $T>0$ from Ref 147 If time-reversal symmetry apphes, then $\beta=1$, but in the presence of a magnetic field strong enough to suppress the cooperon contributions then $\beta=2$ If the spin degeneracy is lifted, $g_{\mathrm{s}}$ is to be replaced by $g_{\mathrm{s}}^{1 / 2}$ 
(Note that this definition of $l_{\mathrm{T}}$ differs by a factor of $(2 \pi)^{1 / 2}$ from that in Ref. 145.) The thermal smearing of the conductance fluctuations is of importance only if phase coherence extends beyond a length scale $l_{\mathrm{T}}\left(\right.$ i.e., if $l_{\phi} \gg l_{\mathrm{T}}$ ). In this case the total energy interval $k_{\mathrm{B}} T$ around the Fermi level that is available for transport is divided into subintervals of width $E_{\mathrm{c}}\left(l_{\phi}\right)=\hbar / \tau_{\phi}$ in which phase coherence is maintained. There is a number $N \approx k_{\mathrm{B}} T / E_{\mathrm{c}}\left(l_{\phi}\right)$ of such subintervals, which we assume to be uncorrelated. The root-mean-square variation $\delta G$ of the conductance is then reduced by a factor $N^{-1 / 2} \approx l_{\mathrm{T}} / l_{\phi}$ with respect to the result (7.10) in the absence of energy averaging. (A word of caution: as discussed in Ref. 145, the assumption of $N$ uncorrelated energy intervals is valid in the $1 \mathrm{D}$ case $W \ll l_{\phi}$ considered here, but not in higher dimensions.) From the foregoing argument it follows that

$$
\delta G=\text { constant } \times \frac{e^{2}}{h} \frac{l_{\mathrm{T}} l_{\phi}^{1 / 2}}{L^{3 / 2}}, \quad \text { if } l_{\phi} \gg l_{\mathbf{T}} .
$$

The asymptotic expressions (7.10) and (7.13) were derived by Lee, Stone, and Fukuyama ${ }^{145}$ and by Al'tshuler and Khmel'nitskii ${ }^{146}$ up to unspecified constant prefactors. These constants have been evaluated in Ref. 147, and are given in Table III. In that paper we also gave an interpolation formula

$$
\delta G=\frac{g_{\mathrm{s}} g_{\mathrm{v}}}{2} \beta^{-1 / 2} \sqrt{12} \frac{e^{2}}{h}\left(\frac{l_{\phi}}{L}\right)^{3 / 2}\left[1+\frac{9}{2 \pi}\left(\frac{l_{\phi}}{l_{\mathrm{T}}}\right)^{2}\right]^{-1 / 2},
$$

with $\beta$ defined in the previous subsection. This formula is valid (within $10 \%$ accuracy) also in the intermediate regime when $l_{\phi} \approx l_{\mathrm{T}}$, and is useful for comparison with experiments, in which generally $l_{\phi}$ and $l_{\mathrm{T}}$ are not well separated (cf. Table I).

\section{c. Magnetoconductance Fluctuations}

Experimentally, one generally studies the conductance fluctuations resulting from a change in Fermi energy $E_{\mathrm{F}}$ or magnetic field $B$ rather than from a change in impurity configuration. A comparison with the theoretical ensemble average becomes possible if one assumes that, insofar as the conductance fluctuations are concerned, a sufficiently large change in $E_{\mathrm{F}}$ or $B$ is equivalent to a complete change in impurity configuration (this "ergodic hypothesis" has been proven in Ref. 148). The reason for this equivalence is that, on one hand, the conductance at $E_{\mathrm{F}}+\Delta E_{\mathrm{F}}$ and $B+\Delta B$ is uncorrelated with that at $E_{\mathrm{F}}$ and $B$, provided either $\Delta E_{\mathrm{F}}$ or $\Delta B$ is larger than a correlation energy $\Delta E_{\mathrm{c}}$ or correlation field $\Delta B_{\mathrm{c}}$. On the other hand, the correlation energies and fields are in general sufficiently small that the statistical properties of the ensemble are not modified by the increment in $E_{\mathrm{F}}$ or $B$, so one is essentially studying a new member of the same ensemble, without changing the sample. 
This subsection deals with the calculation of the correlation field $\Delta B_{\mathrm{c}}$. (The correlation energy is discussed in Ref. 145 and will not be considered here.) The magnetoconductance correlation function is defined as

$$
F(\Delta B) \equiv\langle[\delta G(B)-\langle G(B)\rangle][G(B+\Delta B)-\langle G(B+\Delta B)\rangle]\rangle,
$$

where the angle brackets $\langle\cdots\rangle$ denote, as before, an ensemble average. The root-mean-square variation $\delta G$ considered in the previous two subsections is equal to $F(0)^{1 / 2}$. The correlation field $\Delta B_{\mathrm{c}}$ is defined as the half-width at halfheight $F\left(\Delta B_{\mathrm{c}}\right) \equiv F(0) / 2$. The correlation function $F(\Delta B)$ is determined theoretically ${ }^{141,145,146}$ by temporal and spatial integrals of two propagators: the diffuson $P_{\mathrm{d}}\left(\mathbf{r}, \mathbf{r}^{\prime}, t\right)$ and the cooperon $P_{\mathrm{c}}\left(\mathbf{r}, \mathbf{r}^{\prime}, t\right)$. As discussed by Chakravarty and Schmid, ${ }^{126}$ these propagators consist of the product of three terms: (1) the classical probability to diffuse from $\mathbf{r}$ to $\mathbf{r}^{\prime}$ in a time $t$ (independent of $B$ in the field range $\omega_{\mathfrak{c}} \tau \ll 1$ of interest here); (2) the relaxation factor $\exp \left(-t / \tau_{\phi}\right)$, which describes the loss of phase coherence due to inelastic scattering events; (3) the average phase factor $\langle\exp (i \Delta \phi)\rangle$, which describes the loss of phase coherence due to the magnetic field. The average $\langle\cdots\rangle$ is taken over all classical trajectories that diffuse from $\mathbf{r}$ to $\mathbf{r}^{\prime}$ in a time $t$. The phase difference $\Delta \phi$ is different for a diffuson or cooperon:

$$
\begin{aligned}
\Delta \phi(\text { diffuson }) & =\frac{e}{\hbar} \int_{r}^{r^{\prime}} \Delta \mathbf{A} \cdot d \mathbf{l}, \\
\Delta \phi(\text { cooperon }) & =\frac{e}{\hbar} \int_{r}^{r^{\prime}}(2 \mathbf{A}+\Delta \mathbf{A}) \cdot d \mathbf{l},
\end{aligned}
$$

where the line integral is along a classical trajectory. The vector potential $\mathbf{A}$ corresponds to the magnetic field $\mathbf{B}=\nabla \times \mathbf{A}$, and the vector potential increment $\Delta \mathbf{A}$ corresponds to the field increment $\Delta B$ in the correlation function $F(\Delta B)$ (according to $\Delta \mathbf{B}=\nabla \times \Delta \mathbf{A}$ ). An explanation of the different magnetic field dependencies of the diffuson and cooperon in terms of Feynman paths is given shortly.

In Ref. 109 we have proven that in a narrow channel $\left(W \ll l_{\phi}\right)$ the average phase factor $\langle\exp (i \Delta \phi)\rangle$ does not depend on initial and final coordinates $\mathbf{r}$ and $\mathbf{r}^{\prime}$, provided that one works in the Landau gauge and that $t \gg \tau$. This is a very useful property, since it allows one to transpose the results for $\langle\exp (i \Delta \phi)\rangle$ obtained for $\mathbf{r}=\mathbf{r}^{\prime}$ in the context of weak localization to the present problem of the conductance fluctuations, where $\mathbf{r}$ can be different from $\mathbf{r}^{\prime}$. We recall that for weak localization the phase difference $\Delta \phi$ is that of the cooperon, with the vector potential increment $\Delta \mathbf{A}=0$ [cf. Eq. (6.5)]. The average phase factor then decays exponentially as $\langle\exp (i \Delta \phi)\rangle=\exp \left(-t / \tau_{B}\right)$ [cf. Eq. (6.11)], with the relaxation time $\tau_{B}$ given as a function of magnetic 
field $B$ in Table II We conclude that the same exponential decay holds for the average cooperon and diffuson phase factors after substitution of $B \rightarrow B+\Delta B / 2$ and $B \rightarrow \Delta B / 2$, respectively, in the expressions for $\tau_{B}$

$$
\begin{gathered}
\left\langle e^{i \Delta \phi}\right\rangle \text { (diffuson) }=\exp \left(-t / \tau_{\Delta B / 2}\right), \\
\left\langle e^{i \Delta \phi}\right\rangle \text { (cooperon) }=\exp \left(-t / \tau_{B+\Delta B / 2}\right)
\end{gathered}
$$

The cooperon is suppressed when $\tau_{B+\Delta B / 2} \lesssim \tau_{\phi}$, which occurs on the same field scale as the suppression of weak localization (determined by $\tau_{B} \lesssim \tau_{\phi}$ ) The suppression of the cooperon can be seen as a consequence of the breaking of the tıme-reversal invariance by the magnetic field, similar to the suppression of weak localization In a zero field the cooperons and the diffusons contribute equally to the variance of the conductance, therefore, when the cooperon is suppressed, $\operatorname{Var}(G)$ is reduced by a factor of 2 (The parameter $\beta$ in Table III thus changes from 1 to 2 when $B$ increases beyond $B_{\mathrm{c}}$ ) In general, the magnetoconductance fluctuations are studied for $B>B_{\mathrm{c}}$ ( $1 \mathrm{e}$, for fields beyond the weak localization peak) Then only the diffuson contributes to the conductance fluctuations, since the relaxation time of the diffuson is determined by the field increment $\Delta B$ in the correlation function $F(\Delta B)$, not by the magnetic field itself This is the critical difference with weak localization The conductance fluctuations are not suppressed by a weak magnetic field ${ }^{141146}$ The different behavior of cooperons and diffusons can be understood in terms of Feynman paths The correlation function $F(\Delta B)$ contains the product of four Feynman path amplitudes $A(l, B), A^{*}(J, B)$, $A(k, B+\Delta B)$, and $A^{*}(l, B+\Delta B)$ along various paths $l, \jmath, k, l$ from $\mathbf{r}$ to $\mathbf{r}^{\prime}$ Consider the diffuson term for which $l=l$ and $J=k$ The phase of this term $A(l, B) A^{*}(J, B) A(\jmath, B+\Delta B) A^{*}(l, B+\Delta B)$ is

$$
-\frac{e}{\hbar} \oint \mathbf{A} \cdot d \mathbf{l}+\frac{e}{\hbar} \oint(\mathbf{A}+\Delta \mathbf{A}) \cdot d \mathbf{l}=\frac{e}{\hbar} \Delta \Phi,
$$

where the line integral is taken along the closed loop formed by the two paths $l$ and $J$ (cf Fig 21a) The phase is thus given by the flux increment $\Delta \Phi \equiv S \Delta B$ through this loop and does not contain the flux $\Phi \equiv S B$ itself The fact that the magnetic relaxation time of the diffuson depends only on $\triangle B$ and not on $B$ is a consequence of the cancellation contained in Eq (7 18) For the cooperon, the relevant phase is that of the product of Feynman path amplitudes $A_{-}(l, B) A^{*}(J, B) A_{+}(J, B+\Delta B) A_{+}^{*}(l, B+\Delta B)$, where the - sign refers to a trajectory from $\mathbf{r}^{\prime}$ to $\mathbf{r}$ and the + sign to a trajectory from $\mathbf{r}$ to $\mathbf{r}^{\prime}$ (see Fig 21b) This phase is given by

$$
\frac{e}{\hbar} \oint \mathbf{A} \cdot d \mathbf{l}+\frac{e}{\hbar} \oint(\mathbf{A}+\Delta \mathbf{A}) \cdot d \mathbf{l}=\frac{e}{\hbar}(2 \Phi+\Delta \Phi)
$$


(a)

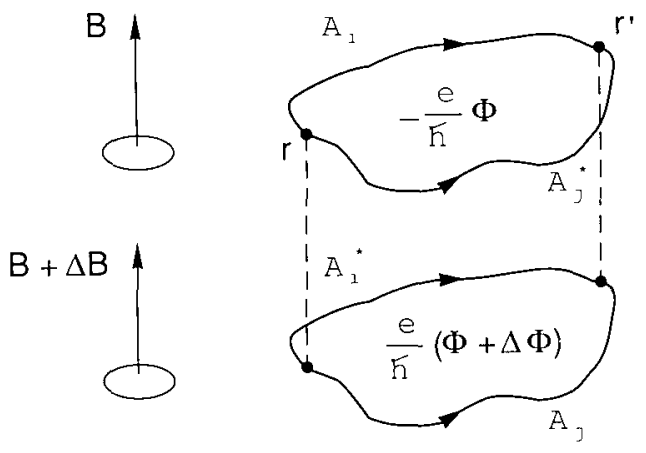

(b)

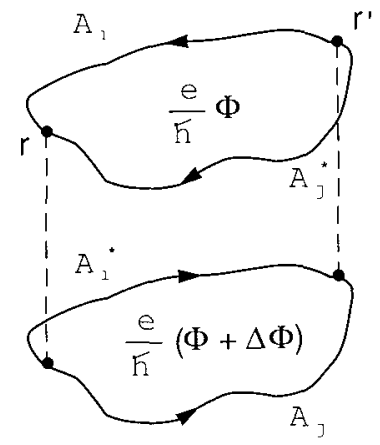

FIG 21 Illustration of the different flux sensitivity of the interference terms of diffuson type (a) and of cooperon type (b). Both contribute to the conductance fluctuations in a zero magnetic field, but the cooperons are suppressed by a weak magnetıc field, as discussed in the text.

In contrast to the diffuson, the cooperon is sensitive to the flux $\Phi$ through the loop and can therefore be suppressed by a weak magnetic field.

In the following, we assume that $B>B_{\mathrm{c}}$ so that only the diffuson contributes to the magnetoconductance fluctuations. The combined effects of magnetic field and inelastic scattering lead to a relaxation rate

$$
\tau_{\text {eff }}^{-1}=\tau_{\phi}^{-1}+\tau_{\Delta B / 2}^{-1}
$$

which describes the exponential decay of the average phase factor $\left\langle e^{i \Delta \phi}\right\rangle=\exp \left(-t / \tau_{\text {eff }}\right)$. Equation (7.20) contains the whole effect of the magnetic field on the diffuson. Without having to do any diagrammatic analysis, we therefore conclude ${ }^{147}$ that the correlation function $F(\Delta B)$ can be obtained from the variance $F(0) \equiv \operatorname{Var} G=(\delta G)^{2}$ (given in Table III) by simply replacing $\tau_{\phi}$ by the effective relaxation time $\tau_{\text {eff }}$ defined in Eq. (7.20). The quantity $\tau_{\Delta B / 2}$ corresponds to the magnetic relaxation time $\tau_{B}$ obtained for weak localization (see Table II) after substitution of $B \rightarrow \Delta B / 2$. For easy reference, we give the results for the dirty and clean metal regimes explicitly: ${ }^{109,147}$

$$
\begin{aligned}
& \tau_{\Delta B / 2}=12\left(\frac{\hbar}{e \Delta B}\right)^{2} \frac{1}{D W^{2}}, \quad \text { if } l \ll W, \\
& \tau_{\Delta B / 2}=4 C_{1}\left(\frac{\hbar}{e \Delta B}\right)^{2} \frac{1}{v_{\mathrm{F}} W^{3}}+2 C_{2}\left(\frac{\hbar}{e \Delta B}\right) \frac{l}{v_{\mathrm{F}} W^{2}}, \quad \text { if } l \gg W,
\end{aligned}
$$

${ }^{147}$ C. W. J. Beenakker and H. van Houten, Phys. Rev. B 37, 6544 (1988). 
where $C_{1}=9.5$ and $C_{2}=24 / 5$ for a channel with specular boundary scattering $\left(C_{1}=4 \pi\right.$ and $C_{2}=3$ for a channel with diffuse boundary scattering). These results are valid under the condition $W^{2} \Delta B \ll \hbar / e$, which follows from the requirement $\tau_{\text {eff }} \gg \tau$ that the electronic motion on the effective phase coherence time scale $\tau_{\text {eff }}$ be diffusive rather than ballistic, as well as from the requirement $\left(D \tau_{\text {eff }}\right)^{1 / 2} \gg W$ for one-dimensionality.

With results (7.20)-(7.22), the equation $F\left(\Delta B_{\mathrm{c}}\right)=F(0) / 2$, which defines the correlation field $\Delta B_{\mathrm{c}}$, reduces to an algebraic equation that can be solved straightforwardly. In the dirty metal regime one finds ${ }^{145}$

$$
\Delta B_{\mathrm{c}}=2 \pi C \frac{\hbar}{e} \frac{1}{W l_{\phi}},
$$

where the prefactor $C$ decreases from ${ }^{147} 0.95$ for $l_{\phi} \gg l_{\mathrm{T}}$ to 0.42 for $l_{\phi} \ll l_{\mathrm{T}}$. Note the similarity with the result (6.9) for weak localization. Just as in weak localization, one finds that the correlation field in the pure metal regime is significantly enhanced above Eq. (7.23) due to the flux cancellation effect discussed in Section 6.c. The enhancement factor increases from $(l / W)^{1 / 2}$ to $l / W$ as $l_{\phi}$ decreases from above to below the length $l^{3 / 2} W^{-1 / 2}$. The relevant expression is given in Ref. 147. As an illustration, the dimensionless correlation flux $\Delta B_{\mathrm{c}} W l_{\phi} e / h$ in the pure and dirty metal regimes is plotted as a function of $l_{\phi} / l$ in Fig. 22 for $l_{\mathrm{T}} \ll l_{\phi}$.

In the following discussion of the experimental situation in semiconductor nanostructures, it is important to keep in mind that the Al'tshuler-LeeStone theory of conductance fluctuations was formulated for an application

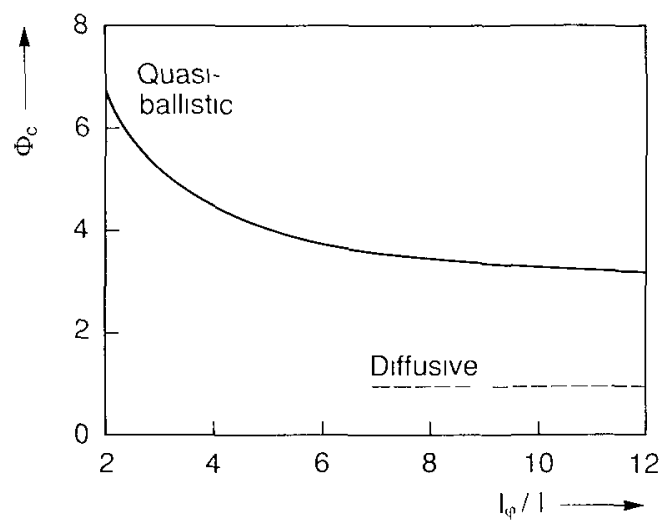

Fig 22. Plot of the dimensionless correlation flux $\Phi_{\mathrm{c}} \equiv \Delta B_{\mathrm{c}} l_{\phi} W e / h$ for the magnetoconductance fluctuations as a function of $l_{\phi} / l$ in the regime $l_{\mathrm{T}} \ll l_{\phi}$ The solid curve 1 f for the case $l=5 \mathrm{~W}$; the dashed line is for $l \ll W$ Taken from C W. J. Beenakker and $H$ van Houten, Phys Rev B 37, $6544(1988)$ 
to metals. This has justified the neglect of several possible complications, which may be important in a 2DEG. One of these is the classical curvature of the electron trajectories, which affects the conductance when $l_{\text {cycl }} \lesssim \min (W, l)$. A related complication is the Landau level quantization, which in a narrow channel becomes important when $l_{\mathrm{m}} \lesssim W$. Furthermore, when $W \sim \lambda_{\mathrm{F}}$ the lateral confinement will at low fields induce the formation of $1 \mathrm{D}$ subbands. No quantization effects are taken into account in the theory of conductance fluctuations discussed before. Finally, the present theory is valid only in the regime of coherent diffusion $\left(\tau_{\phi}, \tau_{\text {eff }} \gtrsim \tau\right)$. In high-mobility samples $\tau_{\phi}$ and $\tau$ may be comparable, however, as discussed in Section 7.d. It would be of interest to study the conductance fluctuations in this regime theoretically.

In the following discussion of experimental studies of conductance fluctuations, we will have occasion to discuss briefly one further development. This is the modification of the theory ${ }^{149-154}$ to account for the differences between two- and four-terminal measurements of the conductance fluctuations, which becomes important when the voltage probes are separated by less than the phase coherence length. ${ }^{155,156}$

\section{d. Experiments}

The experimental observation of conductance fluctuations in semiconductors has preceded the theoretical understanding of this phenomenon. Weak irregular conductance fluctuations in wide $\mathbf{S} 1$ inversion layers were reported in 1965 by Howard and Fang. ${ }^{157}$ More pronounced fluctuations were found by Fowler et al. in narrow Si accumulation layers in the strongly localızed regime. ${ }^{32} \mathrm{~K}$ wasnick et al. made similar observations in narrow $\mathrm{Si}$ inversion layers in the metallic conduction regime. ${ }^{39}$ These fluctuations in the conductance as a function of gate voltage or magnetic field have been tentatively explanned by various mechanisms. ${ }^{158}$ One of the explanations suggested is based on resonant tunneling, ${ }^{159}$ another on variable range hoppıng. ${ }^{160}$ At the 1984 conference on "Electronic Properties of Two-

${ }^{148}$ B L Al'tshuler, V E Kravtsov, and I V Lerner, Pts'ma $Z h$ Eksp Teor Fiz 43, 342 (1986)

[JETP Lett 43, $441(1986)]$

${ }^{149}$ M Buttıer, Phys Rev B 35, 4123 (1987)

${ }^{150} \mathrm{~S}$ Maekawa, Y Isawa, and H Ebisawa, $J$ Phys Soc Jpn 56, 25 (1987)

${ }^{151} \mathrm{H}$ U Baranger, A D Stone, and D P DiVincenzo, Phys Rev B 37, 6521 (1988)

${ }^{152} \mathrm{~S}$ Hershfield and V Ambegaokar, Phys Rev B 38, 7909 (1988)

${ }^{153}$ C L Kane, P A Lee, and D P DiVincenzo, Phys Rev B 38, 2995 (1988)

${ }^{154}$ D P DiVincenzo and C L Kane, Phys Rev B 38, 3006 (1988)

${ }^{155}$ A D Benott, C P Umbach, R B Larbowitz, and R A Webb, Phys Rev Lett 58, 2343(1987)

${ }^{156}$ W J Skocpol, P M Mankiewich, R E Howard, L D Jackel, D M Tennant, and A D

Stone, Phys Rev Lett 58, 2347 (1987)

${ }^{157}$ W E Howard and F F Fang, Sold State Electronics 8, 82 (1965)

${ }^{158}$ A Hartstem, R A Webb, A B Fowler, and J J Wainer, Surf $S_{c l}$ 142, 1 (1984)

${ }^{159} \mathrm{M}$ Ya Azbel, Phys Rev B 28, 4106 (1983)

${ }^{160} \mathrm{P}$ A Lee, Phys Rev Lett 53, $2042(1984)$ 


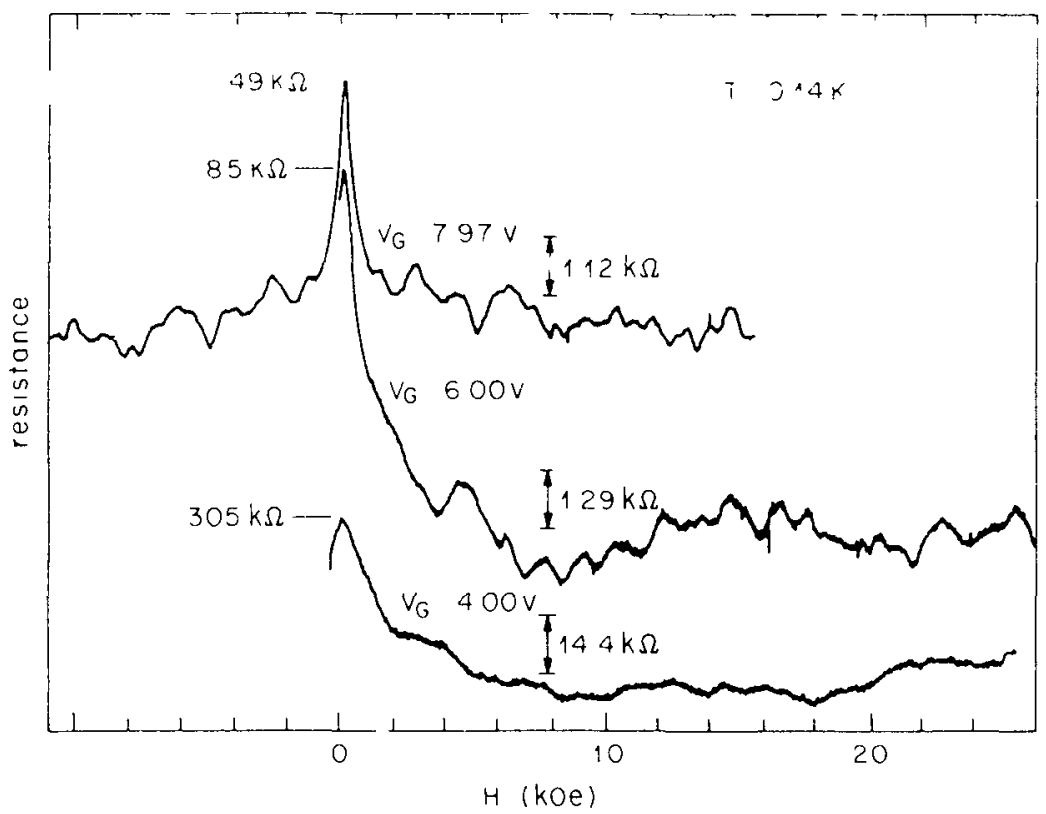

Fig 23 Negative magnetoresistance and aperiodic magnetoresistance fluctuations in a narrow $S i$ inversion layer channel for several values of the gate voltage $V_{\mathrm{G}}$ Note that the vertical offset and scale is different for each $V_{\mathrm{G}}$ Taken from J C Licinı et al, Phys Rev Lett 55, 2987 (1985)

Dimensional Systems" Wheeler et al ${ }^{161}$ and Skocpol et al ${ }^{162}$ reported pronounced structure as a function of gate voltage in the low-temperature conductance of narrow $S i$ inversion layers, observed in the course of their search for a quantum size effect

After the publication in 1985 of the Al'tshuler-Lee-Stone theory ${ }^{140141163}$ of universal conductance fluctuations, a consensus has rapidly developed that this theory properly accounts for the conductance fluctuations in the metallic regime, up to factor of two uncertainties in the quantitative description ${ }^{46144164}$ Following this theoretical work, Licini et $a l^{40}$ attributed the magnetoresistance oscillations that they observed in narrow $S_{1}$ inversion layers to quantum interference in a disordered conductor Their low-temperature measurements, which we reproduce in Fig 23,

${ }^{161} \mathrm{R}$ G Wheeler, K K Cho1, and R Wisnieff, Surf Scl 142, 19 (1984)

${ }^{162}$ W J Skocpol, L D Jackel, R E Howard, H G Craighead, L A Fetter, P M Mankiewıch,

P Grabbe, and D M Tennant, Surf Sct 142, 14 (1984)

${ }^{163}$ A D Stone, Phys Rev Lett 54, 2692 (1985)

${ }^{164} \mathrm{R}$ A Webb, S Washburn, H J Haucke, A D Benort, C P Umbach, and F P Millken, in Ref 14 
show a large negative magnetoresistance peak due to weak localızation at low magnetic fields, in addition to aperiodic fluctuations that persist to high fields Such a clear weak localization peak is not found in shorter samples, where the conductance fluctuations are larger The reason is that the magnitude of the conductance fluctuations $\Delta G$ is proportional to $\left(l_{\phi} / L\right)^{3 / 2}$ [for $l_{\phi} \ll l_{\mathrm{T}}$, cf $\left.\mathrm{Eq}(710)\right]$, while the weak localization conductance correction scales with $l_{\phi} / L$ [as discussed below Eq (64)] Weak localization thus predominates in long channels $\left(L \gg l_{\phi}\right)$ where the fluctuations are relatively unımportant

The most extensive quantitative study of the universality of the conductance fluctuations in narrow Si inversion layers (over a wide range of channel widths, lengths, gate voltages, and temperatures) was made by Skocpol et al ${ }^{4546156}$ In the following, we review some of these experimental results We will not discuss the similarly extensive investigations by Webb et al ${ }^{155164165}$ on small metallic samples, which have played an equally important role in the development of this subject To analyze their exper1ments, Skocpol et al estımated $l_{\phi}$ from weak localization experıments (with an estımated uncertainty of about a factor of 2) They then plotted the rootmean-square variation $\delta G$ of the conductance as a function of $L / l_{\phi}$, with $L$ the separation of the voltage probes in the channel Their results are shown in Fig 24 The points for $L>l_{\phi}$ convincingly exhibit for a large variety of data sets the $\left(L / l_{\phi}\right)^{3 / 2}$ scaling law predicted by the theory described in Section $7 \mathrm{c}$ (for $l_{\phi}<l_{\mathrm{T}}$, which is usually the case in $\mathrm{S}_{1}$ inversion layers)

For $L<l_{\phi}$ the experimental data of $F_{1 g} 24$ show a crossover to a $\left(L / l_{\phi}\right)^{-2}$ scalıng law (dashed line), accompanied by an increase of the magnitude of the conductance fluctuations beyond the value $\delta G \approx e^{2} / h$ predicted by the Al'tshuler-Lee-Stone theory for a conductor of length $L<l_{\phi}$ A similar observation was made by Benoit et al ${ }^{155}$ on metallıc samples The disagreement is explained ${ }^{155156}$ by considering that the experimental geometry differs from that assumed in the theory discussed in Section $7 \mathrm{c}$ Use is made of a long channel with voltage probes at different spacings The experimental $L$ is the spacing of two voltage probes, and not the length of a channel connecting two phase-randomizing reservors, as envisaged theoretically The difference is irrelevant if $L>l_{\phi}$ If the probe separation $L$ is less than the phase coherence length $l_{\phi}$, however, the measurement still probes a channel segment of length $l_{\phi}$ rather than $L$ In this sense the measurement is nonlocal ${ }^{155156}$ The key to the $L^{-2}$ dependence of $\delta G$ found experimentally is that the voltages on the probes fluctuate independently, implying that the

${ }^{165} \mathrm{R}$ A Webb, S Washburn, C P Umbach, and R B Labowitz, in "Localization, Interaction, and Transport Phenomena," p 121 (B Kramer, G Bergmann, and Y Bruynseraede, eds) Sprınger, New York, 1984 


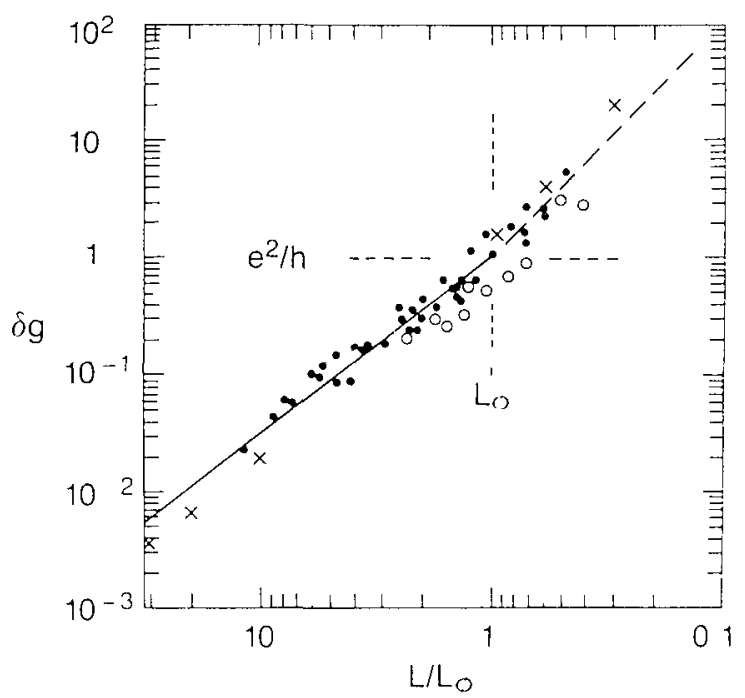

FIG 24 Root-mean-square amplitude $\delta g$ of the conductance fluctuations (in units of $e^{2} / h$ ) as a function of the ratio of the distance between the voltage probes $L$ to the estimated phase coherence length $L_{\phi}$ for a set of Si inversion layer channels under widely varying experimental conditions The solid and dashed lines demonstrate the $\left(L / l_{\phi}\right)^{-3 / 2}$ and $\left(L / l_{\phi}\right)^{-2}$ scaling of $\delta g$ in the regimes $L>l_{\phi}$ and $L<l_{\phi}$, respectively Taken from W J Skocpol, Physica Scripta T19, 95 (1987).

resistance fluctuations $\delta R$ are independent of $L$ in this regime so that $\delta G \approx R^{-2} \delta R \propto L^{-2}$. This explanation is consistent with the anomalously small correlation field $B_{\mathrm{c}}$ found for $L<l_{\phi} \cdot{ }^{46,156}$ One might have expected that the result $B_{\mathrm{c}} \approx \hbar / e W l_{\phi}$ for $L>l_{\phi}$ should be replaced by the larger value $B_{\mathrm{c}} \approx \hbar / e W L$ if $L$ is reduced below $l_{\phi}$. The smaller value found experimentally is due to the fact that the flux through parts of the channel adjacent to the segment between the voltage probes, as well as the probes themselves, has to be taken into account. These qualitative arguments ${ }^{155,156}$ are supported by detailed theoretical investigations. ${ }^{149-154}$ The important message of these theories and experiments is that the transport in a small conductor is phase coherent over large length scales and that phase randomization (due to inelastic collisions) occurs mainly as a result of the voltage probes. The Landauer-Büttiker formalism ${ }^{4,5}$ (which we will discuss in Section 12) is naturally suited to study such problems theoretically. In that formalism, current and voltage contacts are modeled by phase-randomizing reservoirs attached to the conductor. We refer to a paper by Büttiker ${ }^{149}$ for an instructive discussion of conductance fluctuations in a multiprobe conductor in terms of interfering Feynman paths.

Conductance fluctuations have also been observed in narrow-channel 
GaAs-AlGaAs heterostructures ${ }^{166} 167$ These systems are well in the pure metal regime $(W<l)$, but unfortunately they are only marginally in the regime of coherent diffusion (characterized by $\tau_{\phi} \gg \tau$ ) This hampers a quantitative comparison with the theoretical results ${ }^{147}$ for the pure metal regime discussed in Section $7 \mathrm{c}$ (A phenomenological treatment of conductance fluctuations in the case that $\tau_{\phi} \sim \tau$ is given in Refs 168 and 169) The data of Ref 167 are consistent with an enhancement of the correlation field due to the flux cancellation effect, but are not conclusive ${ }^{147}$ We note that the flux cancellation effect can also explain the correlation field enhancement noticed in a computer simulation by Stone ${ }^{163}$

In the analysis of the aforementioned experiments on magnetoconductance fluctuations, a twofold spin degeneracy has been assumed The variance $(\delta G)^{2}$ is reduced by a factor of 2 if the spin degeneracy is lifted by a strong magnetic field $B>B_{\mathrm{c} 2}$ The Zeeman energy $g \mu_{\mathrm{B}} B$ should be sufficiently large than the spin-up and spin-down electrons give statistically independent contributions to the conductance The degeneracy factor $g_{s}^{2}$ in $(\delta G)^{2}$ (introduced in Section 7 a) should then be replaced by a factor $g_{\mathrm{s}}$, since the variances of statıstically independent quantities add Since $g_{\mathrm{s}}=2$, one obtains a factor-of- 2 reduction in $(\delta G)^{2}$ Note that this reduction comes on top of the factor-of-2 reduction in $(\delta G)^{2}$ due to the breaking of time-reversal symmetry, which occurs at weak magnetıc fields $B_{c}$ Stone has calculated ${ }^{170}$ that the field $B_{\mathrm{c} 2}$ in a narrow channel $\left(l_{\phi} \gg W\right)$ is given by the criterion of unit phase change $g \mu_{\mathrm{B}} B \tau_{\phi} / \hbar$ in a coherence time, resulting in the estımate $B_{\mathrm{c} 2} \approx h / g \mu_{\mathrm{B}} \tau_{\phi}$ Surprisingly, the thermal energy $k_{\mathrm{B}} T$ is irrelevant for $B_{\mathrm{c} 2}$ in the $1 \mathrm{D}$ case $l_{\phi} \gg W$ (but not in higher dimensions ${ }^{170}$ )

For the narrow-channel experiment of Ref 167 just discussed, one finds (using the estımates $\tau_{\phi} \approx 7$ ps and $g \approx 04$ ) a crossover field $B_{\mathrm{c} 2}$ of about $2 \mathrm{~T}$, well above the field range used for the data analysis ${ }^{147}$ Most importantly, no magnetoconductance fluctuations are observed of the magnetic field is applied parallel to the 2DEG (see Section 9), demonstratıng that the Zeeman splittıng has no effect on the conductance in this field regime More recently, Debray et al ${ }^{171}$ performed an experımental study of the reduction by a perpendicular magnetic field of the conductance fluctuations as a function of Fermı energy

${ }^{166} \mathrm{~T}$ J Thornton, M Pepper, H Ahmed, G J Davies, and D Andrews, Phys Rev B 36, 4514 (1987)

${ }^{167} \mathrm{H}$ van Houten, B J van Wees, J E Mooly, G Roos, and K -F Berggren, Superlattces and Microstructures 3, 497 (1987)

${ }^{168}$ R P Taylor, M L Leadbeater, G P Wittıngton, P C Main, L Eaves, S P Beaumont, I

McIntyre S Thoms, and C D W Wilkinson, Surf Scl 196, 52 (1988)

${ }^{169}$ T Fukul and H Saito, Jpn J Appl Phys 27 L1320 (1988)

${ }^{170}$ A D Stone, Phys Rev B 39, 10736 (1989)

${ }^{171}$ P Debray, J -L Pichard, J Vicente, and P N Tung, Phys Rev Lett 63, 2264 (1989) 
(varied by means of a gate) The estumated value of $\tau_{\phi}$ ss larger than that of Ref 167 by more than an order of magnitude Consequently, a very small $B_{\mathrm{c} 2} \approx 007 \mathrm{~T}$ is estımated in this experiment The channel is relatively wide ( $2 \mu \mathrm{m}$ lithographic width), so the field $B_{\mathrm{c}}$ for tıme-reversal symmetry breakıng is even smaller $\left(B_{\mathrm{c}} \approx 7 \times 10^{-4} \mathrm{~T}\right)$ A total factor-of-4 reduction in $(\delta G)^{2}$ was found, as expected The values of the observed crossover fields $B_{\mathrm{c}}$ and $B_{\mathrm{c} 2}$ also agree reasonably well with the theoretical prediction Unfortunately, the magnetoconductance in a parallel magnetic field was not investigated by these authors, which would have provided a definitive test for the effect of Zeeman splitting on the conductance above $B_{\mathrm{c} 2}$ We note that related experumental ${ }^{172173}$ and theoretical ${ }^{174175}$ work has been done on the reduction of temporal conductance fluctuations by a magnetic field

The Al'tshuler-Lee-Stone theory of conductance fluctuations ceases to be applicable when the dimensions of the sample approach the mean free path In this ballistic regime observations of large aperıodic, as well as quasi-periodic, magnetoconductance fluctuations have been reported ${ }^{6869139168176-179}$ Quantum interference effects in this regime are determined not by impurity scattering but by scattering off geometrical features of the device, as will be discussed in Section III

\section{Aharonov-Bohm EFFECT}

Magnetoconductance fluctuations in a channel geometry in the diffusive regime are apertodic, since the interfering Feynman paths enclose a continuous range of magnetic flux values A ring geometry, in contrast, encloses a well-defined flux $\Phi$ and thus imposes a fundamental periodicity

$$
G(\Phi)=G(\Phi+n(h / e)), \quad n=1,2,3, \quad,
$$

on the conductance as a function of perpendicular magnetic field $B$ (or flux $\Phi=B S$ through a ring of area $S$ ) Equation (81) expresses the fact that a flux increment of an integer number of flux quanta changes by an integer multiple of $2 \pi$ the phase difference between Feynman paths along the two arms of the ring The periodicity $(81)$ would be an exact consequence of gauge invariance if the magnetic field were nonzero only in the interior of the ring, as in the

${ }^{172}$ N O Birge, B Golding, and W H Haemmerle, Phys Rev Lett 62, 195 (1989)

${ }^{173}$ D Mally, M Sanquer, J -L Pichard, and P Parl, Europhys Lett 8, 471 (1989)

${ }^{174}$ S Feng, P A Lee, and A D Stone, Phys Rev Lett 56, 1960 (1986), erratum 56, 2772 (1986)

${ }^{175}$ B L Altshuler and B Z Spivak, Pls'ma Zh Eksp Teor $F_{l z}$ 42, 363 (1985) [JETP Lett 42, $447(1985)]$

${ }^{176}$ A M Chang, K Owusu-Sekyere, and T Y Chang, Soltd State Comm 67, 1027 (1988)

${ }^{177}$ A M Chang, G Timp, J E Cunnıngham, P M Mankıewıch, R E Behrınger, R E Howard, and $\mathrm{H}$ U Baranger, Phys Rev B 37, 2745 (1988)

${ }^{178} \mathrm{~J}$ A Simmons, D C Tsui, and G Weimann, Surf Scı 196, 81 (1988)

${ }^{179} \mathrm{~S}$ Yamada, H Asa1, Y Fuka1, and T Fuku1, Phys Rev B (to be published) 

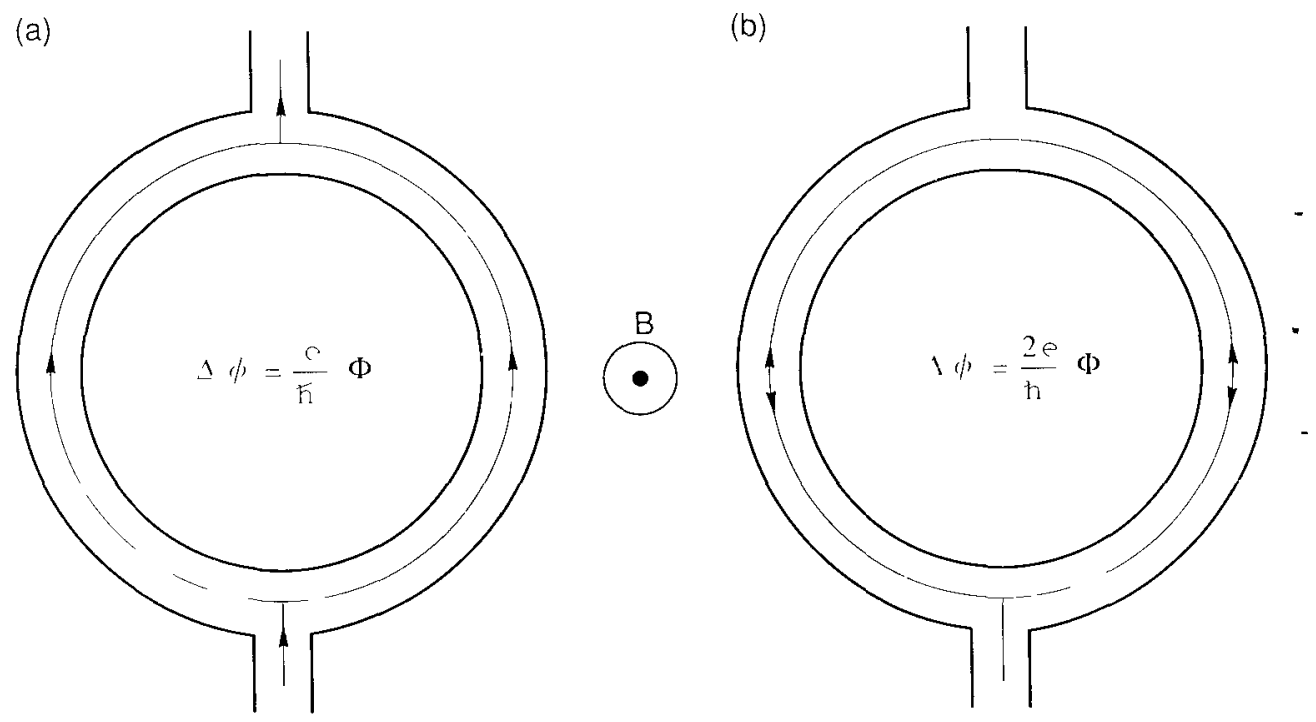

Fig 25 Illustration of the Aharonov-Bohm effect in a ring geometry Interfering trajectories responsible for the magnetoresistance oscillations with $h / e$ periodicity in the enclosed flux $\Phi$ are shown (a) (b) The parr of time-reversed trajectories lead to oscillations with $h / 2 e$ periodicity

original thought experiment of Aharonov and Bohm. ${ }^{180}$ In the present experiments, however, the magnetic field penetrates the arms of the ring as well as its interior so that deviations from Eq. (8.1) can occur. Since in many situations such deviations are small, at least in a limited field range, one still refers to the magnetoconductance oscillations as an Aharonov-Bohm effect.

The fundamental periodicity

$$
\Delta B=\frac{h}{e} \frac{1}{S}
$$

is caused by interference between trajectories that make one half-revolution around the ring, as in Fig. 25a. The first harmonic

$$
\Delta B=\frac{h}{2 e} \frac{1}{S}
$$

results from interference after one revolution. A fundamental distinction between these two periodicities is that the phase of the $h / e$ oscillations (8.2) is sample-specific, whereas the $h / 2 e$ oscillations (8.3) contain a contribution from time-reversed trajectories (as in Fig. 25b) that has a minimum conductance at $B=0$, and thus has a sample-independent phase. Consequently, in a geometry with many rings in series (or in parallel) the $h / e$ oscillations 
average out, but the $h / 2 e$ oscillations remain. The $h / 2 e$ oscllations can be thought of as a perıodic modulation of the weak localization effect due to coherent backscattering.

The first observation of the Aharonov-Bohm effect in the solid state was made by Sharvin and Sharvin ${ }^{181}$ in a long metal cylinder. Since this is effectively a many-ring geometry, only the $h / 2 e$ oscillations were observed, in agreement with a theoretical prediction by Al'tshuler, Aronov, and Spivak, ${ }^{182}$ which motivated the experıment. (We refer to Ref. 125 for a simple estımate of the order of magnitude of the $h / 2 e$ oscillations in the dirty metal regime.) The effect was studied extensively by several groups. ${ }^{183-185}$ The $h / e$ oscillations were first observed in single metal rings by Webb et al. ${ }^{186}$ and studied theoretically by several authors. ${ }^{1,144,187,188}$ The self-averaging of the $h / e$ oscillations has been demonstrated explicitly in experiments with a varying number of rings in series. ${ }^{189}$ Many more experiments have been performed on one- and two-dimensional arrays and networks, as reviewed in Refs. 190 and 191.

In this connection, we mention that the development of the theory of aperiodic conductance fluctuations (discussed in Section 7) has been much stımulated by therr observation in metal rings by Webb et al., ${ }^{165}$ in the course of their search for the Aharonov-Bohm effect. The reason that aperiodic fluctuations are observed in rings (in addition to periodic oscillations) is that the magnetic field penetrates the width of the arms of the ring and is not confined to its interıor. By fabricatıng rings with a large ratio of radius $r$ to width $W$, researchers have proven it is possible to separate ${ }^{190}$ the magnetic field scales of the perodic and aperiodic oscillations (which are given by a field interval of order $h / e r^{2}$ and $h / e W l_{\phi}$, respectively). The penetration of the magnetıc field in the arms of the ring also leads to a broadening of the peak in

${ }^{180} \mathrm{Y}$ Aharonov and D Bohm, Phys Rev 115, 485 (1959)

${ }^{181} \mathrm{D}$ Yu Sharvin and Yu V Sharvin, Pts'ma Zh Teor Fiz 34, 285 (1981) [JETP Lett 34, 272 (1981)

${ }^{182}$ B L Al'tshuler, A G Aronov, and B Z Spivak, Pts'ma Zh Teor Fiz 33, 101 (1981) [JETP Lett 33, 94 (1981)]

${ }^{183}$ B L Al'tshuler, A G Aronov, B Z Spivak, D Yu Sharvin, and Yu V Sharvin, Pis'ma Zh Eksp Teor Flz 35, 476 (1982) [JETP Lett 35, 588 (1982)]

${ }^{184} \mathrm{Yu}$ V Sharvin, Physica B 126, 288 (1984)

${ }^{185} \mathrm{M}$ Gijs, C van Haesendonck, and Y Bruynseraede, Phys Rev Lett 52, 2069 (1984), Phys Rev B 30, 2964 (1984)

${ }^{186} \mathrm{R}$ A Webb, S Washburn, C P Umbach, and R B Larbowitz, Phys Rev Lett 54, 2696 (1985)

${ }^{187}$ Y Gefen, Y Imry, and M Ya Azbel, Surf Sci 142, 203 (1984), Phys Rev Lett 52, 129 (1984)

${ }^{188}$ M Buttıker, Y Imry, R Landauer, and S Pinhas, Phys Rev B 31, 6207 (1985)

${ }^{189} \mathrm{C}$ P Umbach, C Van Haesendonck, R B Labowitz, S Washburn, and R A Webb, Phys Rev Lett 56, 386 (1986)

${ }^{190} \mathrm{~S}$ Washburn and R A Webb, Adv Phys 35, 375 (1986)

${ }^{191}$ A G Aronov and Yu V Sharvin, Rev Mod Phys 59, 755 (1987) 
the Fourier transform at the $e / h$ and $2 e / h$ periodicities, associated with a distribution of enclosed flux ${ }^{192}$ The width of the Fourier peak can be used as a rough estimate for the width of the arms of the ring In addition, the nonzero field in the arms of the ring also leads to a damping of the amplitude of the ensemble-averaged $h / 2 e$ oscillations when the flux through the arms is sufficiently large to suppress weak localization ${ }^{191}$

Two excellent reviews of the Aharonov-Bohm effect in metal rings and cylınders exist ${ }^{190191}$ In the following we discuss the experiments in semiconductor nanostructures in the weak-field regime $\omega_{\mathrm{c}} \tau<1$, where the effect of the Lorentz force on the trajectories can be neglected The strong-field regime $\omega_{\mathrm{c}} \tau>1$ (which is not easily accessible in the usual polycrystalline metal rings) is only briefly mentioned, it is discussed more extensively in Section 21 To our knowledge, no observation of Aharonov-Bohm magnetoresıstance oscillations in $\mathrm{S} 1$ inversion layers has been reported The first observation of the Aharonov-Bohm effect in a 2DEG ring was published by Timp et al, ${ }^{69}$ who employed high-mobility GaAs-AlGaAs heterostructure material Simılar results were obtained independently by Ford et al $^{73}$ and Ishibash 1 et al ${ }^{193}$ More detailed studies soon followed 74139176194195 A characteristic feature of these experiments is the large amplitude of the $h / e$ oscillations (up to $10 \%$ of the average resistance), much higher than in metal rings (where the effect is at best ${ }^{192} 196197$ of order $01 \%$ ) A similar difference in magnitude is found for the aperiodic magnetoresistance fluctuations in metals and semiconductor nanostructures The reason is simply that the amplitude $\delta G$ of the periodic or aperiodic conductance oscillations has a maximum value of order $e^{2} / h$, so the maximum relative resistance oscillation $\delta R / R \approx R \delta G \approx R e^{2} / h$ is proportional to the average resistance $R$, which is typically much smaller in metal rings

In most studies only the $h / e$ fundamental periodicity is observed, although Ford et $a l^{73}{ }^{74}$ found a weak $h / 2 e$ harmonic in the Fourier transform of the magnetoresistance data of a very narrow ring It is not quite clear whether this harmonic is due to the Al'tshuler-Aronov-Spivak mechanısm involving the constructive interference of two time-reversed trajectories ${ }^{182}$ or to the random interference of two non-time-reversed Feynman paths winding around the entire ring ${ }^{1144187}$ The relative weakness of the $h / 2 e$ effect in single $2 \mathrm{DEG}$ rings is also typical for most experiments on single metal rings

\footnotetext{
${ }^{192}$ R A Webb, A Hartstein, J J Wainer, and A B Fowler, Phys Rev Lett 54, 1577 (1985)

${ }^{193} \mathrm{~K}$ Ishıbashı Y Takagakı, K Gamo, S Namba, S Ishıda, K Murase, Y Aoyagı, and M Kawabe, Soltd State Comm 64, 573 (1987)

${ }^{194}$ A M Chang, G Timp, T Y Chang, J E Cunnıngham, B Chellurı, P M Mankıewıch, R E Behringer, and R E Howard, Surf Scl 196, 46 (1988)

${ }^{195}$ C J B Ford, T J Thornton, R Newbury, M Pepper, H Ahmed, D C Peacock, D A

Ritchie, J E F Frost, and G A C Jones, Appl Phys Lett 54, 21 (1989)

${ }^{196}$ C P Umbach, S Washburn, R B Laibowitz, and R A Webb, Phys Rev B 30, 4048 (1984)

${ }^{197}$ V Chandrasekhar, M J Rooks, S Wind, and D E Prober, Phys Rev Lett 55, 1610 (1985)
} 
(although the opposite was found to be true in the case of aluminum rings by Chandrasekhar et al,${ }^{197}$ for reasons which are not understood) This is in contrast to the case of arrays or cylınders, where, as we mentioned, the $h / 2 e$ oscillations are predominant - the $h / e$ effect being "ensemble-averaged" to zero because of its sample-specific phase In view of the fact that the experiments on 2DEG rings explore the borderline between diffusive and ballistic transport, they are rather difficult to analyze quantitatively $\mathrm{A}$ theoretical study of the Aharonov-Bohm effect in the purely ballistic transport regime was performed by Datta and Bandyopadhyay, ${ }^{198}$ in relation to an experımental observation of the effect in a double-quantumwell device ${ }^{199} \mathrm{~A}$ related study was published by Barker ${ }^{200}$

The Aharonov-Bohm oscillations in the magnetoresistance of a small ring in a high-mobility 2DEG are quite impressive As an illustration, we reproduce in Fig 26 the results obtained by Timp et al ${ }^{201}$ Low-frequency modulations were filtered out, so that the rapid oscillations are superimposed on a constant background The amplitude of the $h / e$ oscillations diminishes with increasing magnetic field until eventually the Aharonov-Bohm effect is completely suppressed The reduction in amplitude is accompanied by a reduction in frequency A similar observation was made by Ford et al ${ }^{74}$ In metals, in contrast, the Aharonov-Bohm oscillations persist to the highest experimental fields, with constant frequency The different behavior in a 2DEG is a consequence of the effect of the Lorentz force on the electrons in the ring, which is of importance when the cyclotron diameter $2 l_{\text {cycl }}$ becomes smaller than the width $W$ of the arm of the ring, provided $(W<l)$ (note that $l_{\text {cycl }}=\hbar k_{\mathrm{F}} / e B$ is much smaller in a $2 \mathrm{DEG}$ than in a metal, at the same magnetic field value) We will return to these effects in Section 21

An electrostatic potential $V$ affects the phase of the electron wave function through the term $(e / \hbar) \int V d t$ in much the same way as a vector potential does ${ }^{180}$ If the two arms of the ring have a potential difference $V$, and an electron traverses an arm in a time $t$, then the acquired phase shift would lead to oscillations in the resistance with periodicity $\Delta V=h / e t$ The electrostatic Aharonov-Bohm effect has a periodicity that depends on the transit time $t$, and is not a geometrical property of the ring, as it is for the magnetic effect A distribution of transit times could easily average out the oscillations Note that the potential difference effectuates the phase difference by changing the wavelength of the electrons (via a change in their kinetic energy), which also distinguishes the electrostatic from the magnetic effect (where a phase shift is

\footnotetext{
${ }^{198}$ S Datta and S Bandyopadhyay, Phys Rev Lett 58, 717 (1987)

${ }^{199} \mathrm{~S}$ Datta, M R Melloch, S Bandyopadhyah, $\mathrm{R}$ Noren, M Vazırı, M Miller, and $\mathrm{R}$ Reffenberger, Phys Rev Lett 55, 2344 (1985)

${ }^{200} \mathrm{~J}$ R Barker, in Ref 15

${ }^{201}$ G Timp A M Chang, P DeVegvar, R E Howard, R Behringer, J E Cunnıngham, and P Mankiewich Sulf Scı 19668 (1988)
} 


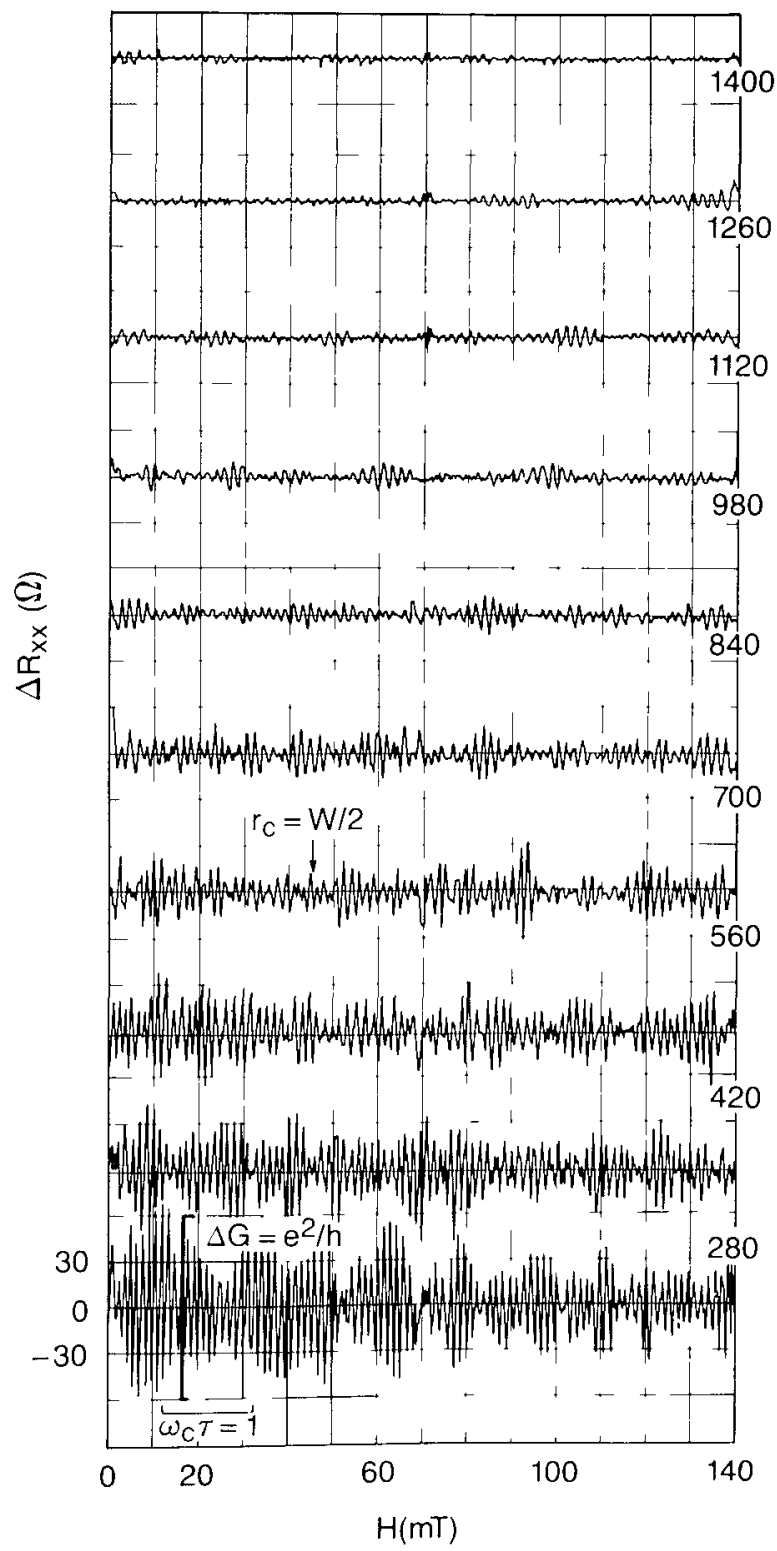

Fig 26 Experımental magnetoresistance of a ring of $2 \mu \mathrm{m}$ diameter, defined in the 2DEG of a high-mobility GaAs-AlGaAs heterostructure $(T=270 \mathrm{mK})$ The different traces are consecutive parts of a magnetoresistance measurement from 0 to $14 \mathrm{~T}$, digitaliy filtered to suppress a slowly varying background The oscillations are seen to persist for fields where $\omega_{\mathrm{c}} \tau>1$, but their amplitude 15 reduced substantially for magnetıc fields where $2 l_{\text {cycl }} \ll W$ (The field value where $2 l_{\text {cycl }} \equiv 2 r_{\mathrm{c}}=W$ is indicated) Taken from G Timp et al, Surf Scl 196, 68 (1988) 
induced by the vector potential without a change in wavelength) An experimental search for the electrostatic Aharonov-Bohm effect in a small metal ring was performed by Washburn et al ${ }^{202}$ An electric field was applied in the plane of the ring by small capacitive electrodes They were able to shift the phase of the magnetoresistance oscillations by varying the field, but the effect was not sufficiently strong to allow the observation of purely electrostatic oscillations Unfortunately, this experıment could not discriminate between the effect of the electric field penetrating in the arms of the ring (which could induce a phase shift by changing the trajectories) and that of the electrostatic potentaal Experıments have been reported by De Vegvar et al ${ }^{203}$ on the manipulation of the phase of the electrons by means of the voltage on a gate electrode positioned across one of the arms of a heterostructure ring In this system a change in gate voltage has a large effect on the resistance of the ring, primarily because it strongly affects the local density of the electron gas No clear periodic signal, indicative of an electrostatic Aharonov-Bohm effect, could be resolved As discussed in Ref 203, this is not too surprising, in view of the fact that in that device 1D subband depopulation in the region under the gate occurs on the same gate voltage scale as the expected Aharonov Bohm effect The observation of an electrostatic AharonovBohm effect thus remains an experımental challenge A successful experıment would appear to require a ring in which only a single $1 \mathrm{D}$ subband is occupied, to ensure a unique transit tıme 198200

\section{Electron-Electron Interactions}

\section{a Theory}

In addition to the weak localization correction to the conductivity discussed in Section 6, which arises from a single-electron quantum interference effect, the Coulomb interaction of the conduction electrons gives also rise to a quantum correction ${ }^{204}{ }^{205}$ In two dimensions the latter correction has a logarithmic temperature dependence, just as for weak localization [see Eq (64)] A perpendicular magnetic field can be used to distınguish the two quantum corrections, which have a different field dependence ${ }^{118} 204-210$

${ }^{202}$ S Washburn, H Schmıd, D Kern, and R A Webb, Phys Rev Lett 59, 1791 (1987)

${ }^{203}$ P G N de Vegvar, G Timp P M Mankiewich, R Behringer, and J Cunningham, Phys Rev B 40, 3491 (1989)

${ }^{204}$ B L Al'tshuler A G Aronov and P A Lee, Phys Rev Lett 44, 1288 (1980)

${ }^{205}$ H Fukuyama, $J$ Phys Soc Japan, 48, 2169 (1980)

${ }^{206}$ B L Al'tshuler, A G Aronov, A I Larkın, and D E Khmel'nitskı1, Zh Eksp Teor Fiz 81, 768 (1981) [Sov Phys JETP 54, 411 (1981)]

${ }^{207}$ B L Al'tshuler, and A G Aronov, Soltd State Comm 46, 429 (1983)

${ }^{208}$ E Abrahams P W Anderson, P A Lee, and T V Ramakrıshnan, Phys Rev B 24, 6783 (1981)

${ }^{209}$ H Fukuyama, $J$ Phys Soc Japan, 50, 3407, 3562 (1981), 51, 1105 (1982)

${ }^{210} \mathrm{P}$ A Lee and T V Ramakrishnan, Phys Rev B 26, 4009 (1982) 
This field of research has been reviewed in detall by Al'tshuler and Aronov, ${ }^{211}$ by Fukuyama, ${ }^{212}$ and by Lee and Ramakrishnan, ${ }^{127}$ with an emphasis on the theory A broader review of electronic correlation effects in 2D systems has been given by Isihara in this series ${ }^{213}$ In the present subsection we summarize the relevant theory, as a preparation for the following subsection on experimental studies in semiconductor nanostructures We do not discuss the diagrammatic perturbation theory, since it is highly technical and does not lend itself to a discussion at the same level as for the other subjects dealt with in this review

An attempt at an intuitive interpretation of the Feynman diagrams was made by Bergmann ${ }^{214}$ It is argued that one important class of diagrams may be interpreted as diffraction of one electron by the oscillations in the electrostatic potential generated by the other electrons The Coulomb interaction between the electrons thus introduces a purely quantum mechanical correlation between their motion, which is observable in the conductivity The diffraction of one electron wave by the interference pattern generated by another electron wave will only be of importance if their wavelength difference, and thus their energy difference, is small At a finite temperature $T$, the characteristic energy difference is $k_{\mathrm{B}} T$ The time $\tau_{\mathrm{T}} \equiv \hbar / k_{\mathrm{B}} T$ enters as a long-time cutoff in the theory of electron-electron interactions in a disordered conductor, in the usual case ${ }^{127211} \tau_{\mathrm{T}} \lesssim \tau_{\phi}$ (Fukuyama $^{212}$ also discusses the opposite limit $\tau_{\mathrm{T}} \gg \tau_{\phi}$ ) Accordingly, the magnitude of the thermal length $l_{\mathrm{T}} \equiv\left(D \tau_{\mathrm{T}}\right)^{1 / 2}$ compared with the width $W$ determines the dimensional crossover from 2D to $1 \mathrm{D}$ [for $\left.l_{\mathrm{T}}<l_{\phi} \equiv\left(D \tau_{\phi}\right)^{1 / 2}\right]$ In the expression for the conductivity correction associated with electron-electron interactions, the long-time cutoff $\tau_{\mathbf{T}}$ enters logarithmically in $2 \mathrm{D}$ and as a square root in 1D These expressions thus have the same form as for weak localization, but with the phase coherence time $\tau_{\phi}$ replaced by $\tau_{\Upsilon}$ The origin of this difference is that a finite temperature does not introduce a long-tıme cutoff for the single-electron quantum interference effect responsible for weak localization, but merely induces an energy average of the corresponding conductivity correction

In terms of effective interaction parameters $g_{2 \mathrm{D}}$ and $g_{1 \mathrm{D}}$, the conductivity

${ }^{211}$ B L Al'tshuler and A G Aronov, in "Electron-Electron Interactions in Disordered Systems," p 1 (A L Efros and M Pollak, eds) North-Holland, Amsterdam, 1985

${ }^{212}$ H Fukuyama, in "Electron-Electron Interactions in Disordered Systems," p 155 (A L Efros and M Pollak, eds ) North-Holland, Amsterdam, 1985

${ }^{213}$ A Isthard, Solıd State Physics, Vol 42, p 271 (H Ehrenreich and D Turnbull, eds) Academic Press, New York, 1989

${ }^{214} \mathrm{G}$ Bergmann, Phys Rev B 35, 4205 (1987) 
corrections due to electron-electron interactions can be written as (assuming $\left.\tau \ll \tau_{\mathbf{T}} \ll \tau_{\phi}\right)$

$$
\begin{array}{ll}
\delta \sigma_{\mathrm{ee}}=-\frac{e^{2}}{2 \pi^{2} \hbar} g_{2 \mathrm{D}} \ln \frac{\tau_{\mathrm{T}}}{\tau}, & \text { for } l_{\mathrm{T}} \ll W, \\
\delta \sigma_{\mathrm{ee}}=-\frac{e^{2}}{2^{1 / 2} \pi \hbar} g_{1 \mathrm{D}} \frac{l_{\mathrm{T}}}{W}, & \text { for } W \ll l_{\mathrm{T}} \ll L .
\end{array}
$$

Under typical experimental conditions, ${ }^{55}$ the constants $g_{2 \mathrm{D}}$ and $g_{1 \mathrm{D}}$ are positive and of order unity. Theoretically, these effective interaction parameters depend in a complicated way on the ratio of screening length to Fermi wavelength and can have either sign. We do not give the formulas here, but refer to the reviews by Al'tshuler and Aronov ${ }^{211}$ and Fukuyama. ${ }^{212}$ In 2D the interaction correction $\delta \sigma_{\mathrm{ee}}$ shares a logarithmic temperature dependence with the weak localization correction $\delta \sigma_{\mathrm{loc}}$, and both corrections are of the same order of magnitude. In 1D the temperature dependences of the two effects are different (unless $\tau_{\phi} \propto T^{-1 / 2}$ ). Moreover, in the 1D case $\delta \sigma_{\mathrm{ec}} \ll \delta \sigma_{\mathrm{loc}}$ if $l_{\Upsilon} \ll l_{\phi}$.

A weak magnetic field fully suppresses weak localization, but has only a small effect on the quantum correction from electron-electron interactions. The conductance correction $\delta G_{\mathrm{ee}}$ contains contributions of diffuson type and of cooperon type. The diffusons (which give the largest contributions to $\delta G_{\mathrm{ee}}$ ) are affected by a magnetic field only via the Zeeman energy, which removes the spin degeneracy when $g \mu_{\mathrm{B}} B \gtrsim k_{\mathrm{B}} T$. In the systems of interest here, spin splitting can usually be ignored below $1 \mathrm{~T}$, so the diffusons are insensitive to a weak magnetic field. Since the spin degeneracy is removed regardless of the orientation of the magnetic field, the $B$-dependence of the diffuson is isotropic. The smaller cooperon contributions exhibit a similar sensitivity as weak localization to a weak perpendicular magnetic field, the characteristic field being determined by $l_{\mathrm{m}}^{2} \approx l_{\mathrm{T}}^{2}$ in $2 \mathrm{D}$ and by $l_{\mathrm{m}}^{2} \approx W l_{\mathrm{T}}$ in $1 \mathrm{D}$ (in the dirty metal regime $W \gg l$, so flux cancellation does not play a significant role). The magnetic length $l_{\mathrm{m}} \equiv\left(\hbar / e B_{\perp}\right)^{1 / 2}$ contains only the component $B_{\perp}$ of the field perpendicular to the $2 \mathrm{DEG}$, since the magnetic field affects the cooperon via the phase shift induced by the enclosed flux. The anisotropy and the small characteristic field are two ways to distinguish experimentally the cooperon contribution from that of the diffuson. It is much more difficult to distinguish the cooperon contribution to $\delta G_{\text {ee }}$ from the weak localization correction, since both effects have the same anisotropy, while their characteristic fields are comparable $\left(l_{\mathrm{T}}\right.$ and $l_{\phi}$ not being widely separated in the systems considered here). This complication is made somewhat less problematic by the fact that the cooperon contribution to $\delta G_{\mathrm{ee}}$ is often considerably smaller 
than $\delta G_{\text {loc }}$, in which case it can be ignored. In $1 \mathrm{D}$ the reduction factor ${ }^{55,211}$ is of order $\left[1+\lambda \ln \left(E_{\mathrm{F}} / k_{\mathrm{B}} T\right)\right]^{-1}\left(l_{\mathrm{T}} / l_{\phi}\right)$, with $\lambda$ a numerical coefficient of order unity.

There is one additional aspect to the magnetoresistance due to electronelectron interactions that is of little experimental relevance in metals but becomes important in semiconductors in the classically strong-field regime where $\omega_{\mathrm{c}} \tau>1$ (this regime is not easily accessible in metal nanostructures because of the typically short scattering time). In such strong fields only the diffuson contributions to the conductivity corrections survive. According to Houghton et al. ${ }^{215}$ and Girvin et al., ${ }^{216}$ the diffuson does not modify the offdiagonal elements of the conductivity tensor, but only the diagonal elements

$$
\delta \sigma_{x y}=\delta \sigma_{y x}=0, \quad \delta \sigma_{x x}=\delta \sigma_{y y} \equiv \delta \sigma_{\mathrm{ec}},
$$

where $\delta \sigma_{\mathrm{ec}}$ is approximately field-independent (provided spin splitting does not play a role). In a channel geometry one measures the longitudinal resistivity $\rho_{x x}$, which is related to the conductivity tensor elements by

$\rho_{x x} \equiv \frac{\sigma_{y y}}{\sigma_{x x} \sigma_{y y}+\sigma_{x y}^{2}}=\rho_{x x}^{0}+\rho_{x x}^{0}\left(\frac{\delta \sigma_{\mathrm{ee}}}{\sigma_{x x}^{0}}-2 \rho_{x x}^{0} \delta \sigma_{\mathrm{ee}}\right)+\operatorname{order}\left(\delta \sigma_{\mathrm{ec}}\right)^{2}$.

Here $\rho_{x x}^{0}=\rho$ and $\sigma_{x x}^{0}=\sigma\left[1+\left(\omega_{\mathrm{c}} \tau\right)^{2}\right]^{-1}$ are the classical results (4.25) and (4.26). In obtaining this result the effects of Landau level quantization on the conductivity have been disregarded (see, however, Ref. 55). The longitudinal resistivity thus becomes magnetic-field-dependent:

$$
\rho_{x x}=\rho\left(1+\left[\left(\omega_{\mathrm{c}} \tau\right)^{2}-1\right] \delta \sigma_{\mathrm{ec}} / \sigma\right) .
$$

To the extent that the $B$-dependence of $\delta \sigma_{\mathrm{ee}}$ can be neglected, Eq. (9.4) gives a parabolic negative magnetoresistance, with a temperature dependence that is that of the negative conductivity correction $\delta \sigma_{\mathrm{ee}}$. This effect can easily be studied up to $\omega_{\mathrm{c}} \tau=10$, which would imply an enhancement by a factor of 100 of the resistivity correction in zero magnetic field. (The Hall resistivity $\rho_{x y}$ also contains corrections from $\delta \sigma_{\mathrm{ee}}$, but without the enhancement factor.) In 2D it is this enhancement that allows the small effect of electron-electron interactions to be observable experimentally (in as far as the effect is due to diffuson-type contributions).

Experimentally, the parabolic negative magnetoresistance associated with electron-electron interactions was first identified by Paalanen et al. ${ }^{137}$ in high-mobility GaAs-AIGaAs heterostructure channels. A more detailed study was made by Choi et al. ${ }^{55}$ In that paper, as well as in Ref.113, it was found that the parabolic magnetoresistance was less pronounced in narrow 
channels than in wider ones. Choi et al. attributed this suppression to specular boundary scattering. It should be noted, however, that specular boundary scattering has no effect at all on the classical conductivity tensor $\boldsymbol{\sigma}^{0}$ (in the scattering time approximation; cf. Section 5.b). Since the parabolic magnetoresistance results from the $\left(\omega_{c} \tau\right)^{2}$ term in $1 / \sigma_{x x}^{0}$ [see Eq. (9.4)], one would expect that specular boundary scattering does not suppress the parabolic magnetoresistance (assuming that the result $\delta \sigma_{x y}=\delta \sigma_{y x}=0$ still holds in the pure metal regime $l>W$ ). Diffuse boundary scattering does affect $\boldsymbol{\sigma}^{\circ}$, but only for relatively weak fields such that $2 l_{\text {cycl }} \gtrsim W$ (see Section 5 ); hence, diffuse boundary scattering seems equally inadequate in explaining the observations. In the absence of a theory for electron-electron interaction effects in the pure metal regime, this issue remains unsettled.

\section{b. Narrow-Channel Experiments}

Wheeler $e t a l .^{38}$ were the first to use magnetoresistance experiments as a tool to distinguish weak localization from electron-electron interaction effects in narrow Si MOSFETs. As in most subsequent studies, the negative magnetoresistance was entirely attributed to the suppression of weak localization; the cooperon-type contributions from electron-electron interactions were ignored. After subtraction of the weak localization correction, the remaining temperature dependence was found to differ from the simple $T^{-1 / 2}$ dependence predicted by the theory for $W<l_{\mathrm{T}}<l_{\phi}$ [Eq. (9.1b)]. This was attributed in Ref. 38 to temperature-dependent screening at the relatively high temperatures of the experiment. Pooke et al. ${ }^{138}$ found a nice $T^{-1 / 2}$ dependence in similar experiments at lower temperatures in narrow $\mathrm{Si}$ accumulation layers and in $\mathrm{GaAs}-\mathrm{AlGaAs}$ heterostructures.

The most detailed study by far of the 2D to $1 \mathrm{D}$ crossover of the electronelectron interaction effect in narrow channels was made by Choi et al. ${ }^{55}$ in a GaAs-AlGaAs heterostructure. In Fig. 27 we reproduce some of their experimental traces for channel widths from 156 to $1.1 \mu \mathrm{m}$ and a channel length of about $300 \mu \mathrm{m}$. The weak localization peak in the magnetoresistance is not resolved in this experiment, presumably because the channels are not in the 1D regime for this effect (the 2D weak localization peak would be small and would have a width of $10^{-4} \mathrm{~T}$ ). The negative magnetoresistance that they found below $0.1-0.2 \mathrm{~T}$ in the narrowest channels is temperature-independent between 1 and $4 \mathrm{~K}$ and was therefore identified by Choi et al. ${ }^{55}$ as a classical size effect. The classical negative magnetoresistance extends over a field range for which $2 l_{\text {cycl }} \gtrsim W$. This effect has been discussed in Section 5 in terms of reduction of backscattering by a magnetic field. The electron-electron interaction effect is observed as a (temperature-dependent) parabolic negative magnetoresistance above $0.1 \mathrm{~T}$ for the widest channel and above $0.3 \mathrm{~T}$ for the narrowest one. From the magnitude of the parabolic negative magnetoresis- 


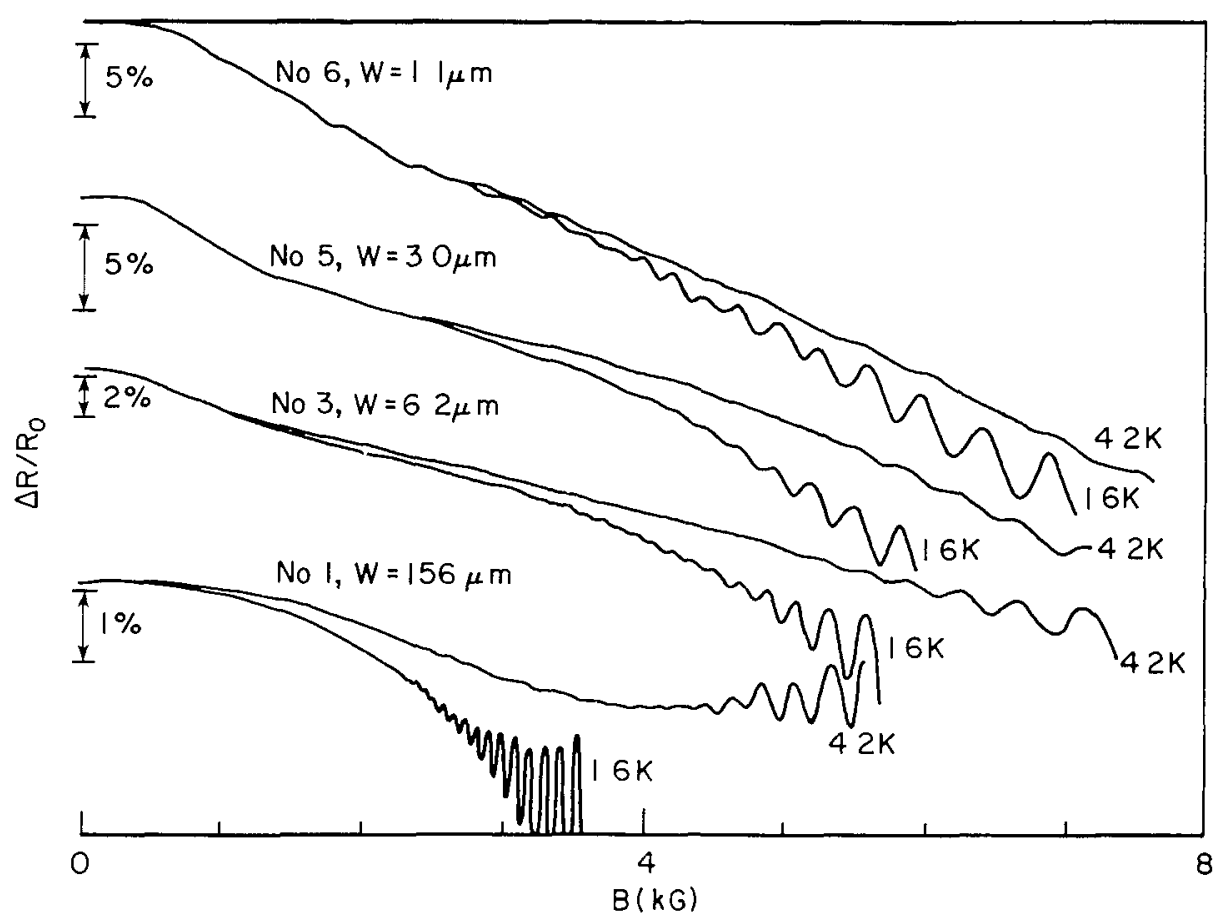

Fig 27 Negative magnetoresistance in wide and narrow GaAs-AlGaAs channels at 42 and $16 \mathrm{~K}$ The temperature-independent negative magnetoresistance at low fields is a classical size effect The temperature-dependent parabolic magnetoresistance at higher fields is a quantum interference effect associated with electron-electron interactions Shubnikov-De Haas oscillations are visible for fields greater than about $03 \mathrm{~T}$ Taken from K K Chos et al, Phys Rev B 33, $8216(1986)$

tance, Choi et al. ${ }^{55}$ could find and analyze the crossover from $2 \mathrm{D}$ to $1 \mathrm{D}$ interaction effects. In addition, they investigated the cross over to $0 \mathrm{D}$ by performing experiments on short channels. As seen in Fig. 27, Shubnikov-De Haas oscillations are superimposed on the parabolic negative magnetoresistance at low temperatures and strong magnetic fields. It is noteworthy that stronger fields are required in narrower channels to observe the ShubnikovDe Haas oscillations, an effect discussed in terms of specular boundary scatterıng by Choi et al. The Shubnikov-De Haas oscillations in narrow channels are discussed further in Section 10.b.

In Refs. 63,167 , and 27 the work by Choi et al. ${ }^{55}$ was extended to even narrower channels, well into the $1 \mathrm{D}$ pure metal regime. The results for a conductıng channel width of $0.12 \mu \mathrm{m}$ are shown in Fig. 28. The 1D weak localization peak in the magnetoresistance is quite large for this narrow 


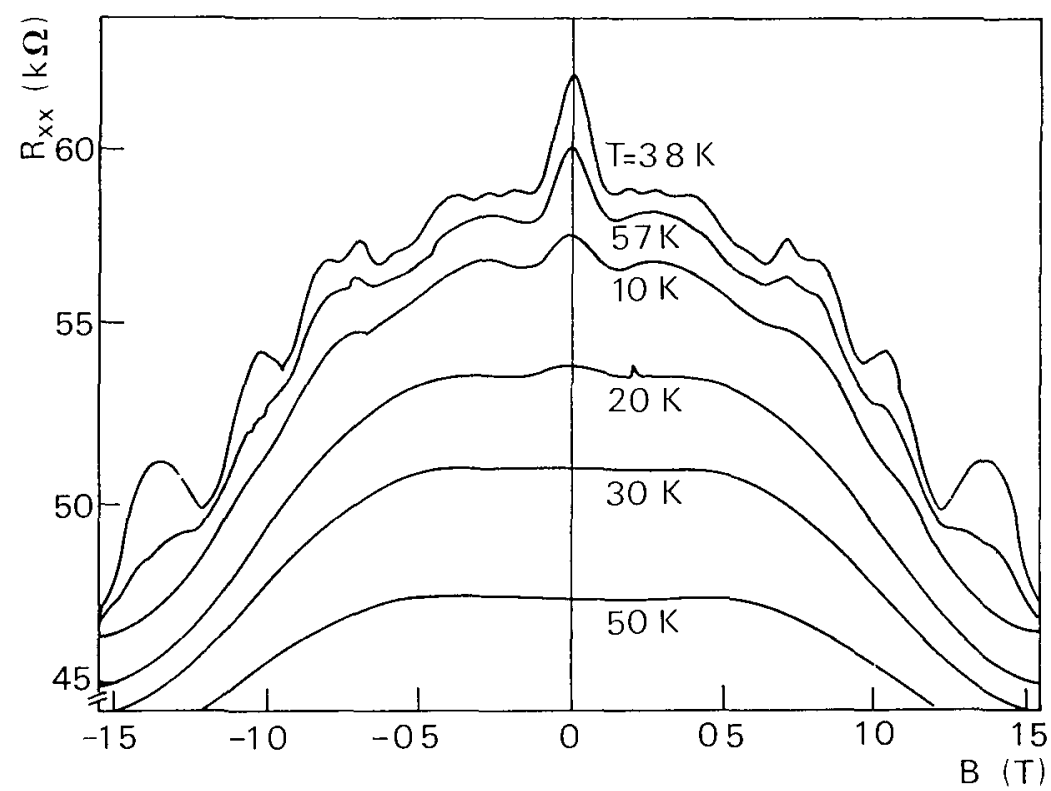

FIG 28 Magnetoresistance at vanous temperatures of a GaAs-AlGaAs channel ( $W=012 \mu \mathrm{m}, L=10 \mu$ ) defined by a shallow-mesa etch technique The central negative magnetoressstance peak between -01 and $+0.1 \mathrm{~T}$ at low temperatures is due to $1 \mathrm{D}$ weak localization in the quasi-ballistic regime Conductance fluctuations are seen at larger fields The negative magnetoresistance that persists to high temperatures is a classical size effect as in Fig 27 The temperature dependence of the resistance at $B=0$ is due to a combination of weak localization and electron-electron interaction effects (see Fig 30) Taken from $\mathrm{H}$ van Houten et al, Appl Phys Lett 49, 1781 (1986)

channel (even at the rather high temperatures shown) and clearly visible below $0.1 \mathrm{~T}$. The classical size effect due to reduction of backscattering now leads to a negative magnetoresistance on a larger field scale of about $1 \mathrm{~T}$, in agreement with the criterion $2 l_{\text {cycl }} \sim W$. This is best seen at temperatures above $20 \mathrm{~K}$, where the quantum mechanical effects are absent. The temperature-dependent parabolic negative magnetoresistance is no longer clearly distınguishable in the narrow channel of Fig. 28, in contrast to wider channels. ${ }^{27,55}$ The suppression of this effect in narrow channels is not yet understood (see Section 9.a). Superimposed on the smooth classical magnetoresistance, one sees large aperiodic fluctuations on a field scale of the same magnitude as the width of the weak localization peak, in qualitative agreement with the theory of universal conductance fluctuations in the pure metal regime ${ }^{147}$ (see Section 7.d). Finally, Shubnikov-De Haas oscillations are beginning to be resolved around $1.2 \mathrm{~T}$, but they are periodic in $1 / B$ at stronger magnetic fields only (not shown). As discussed in Section 10, this 


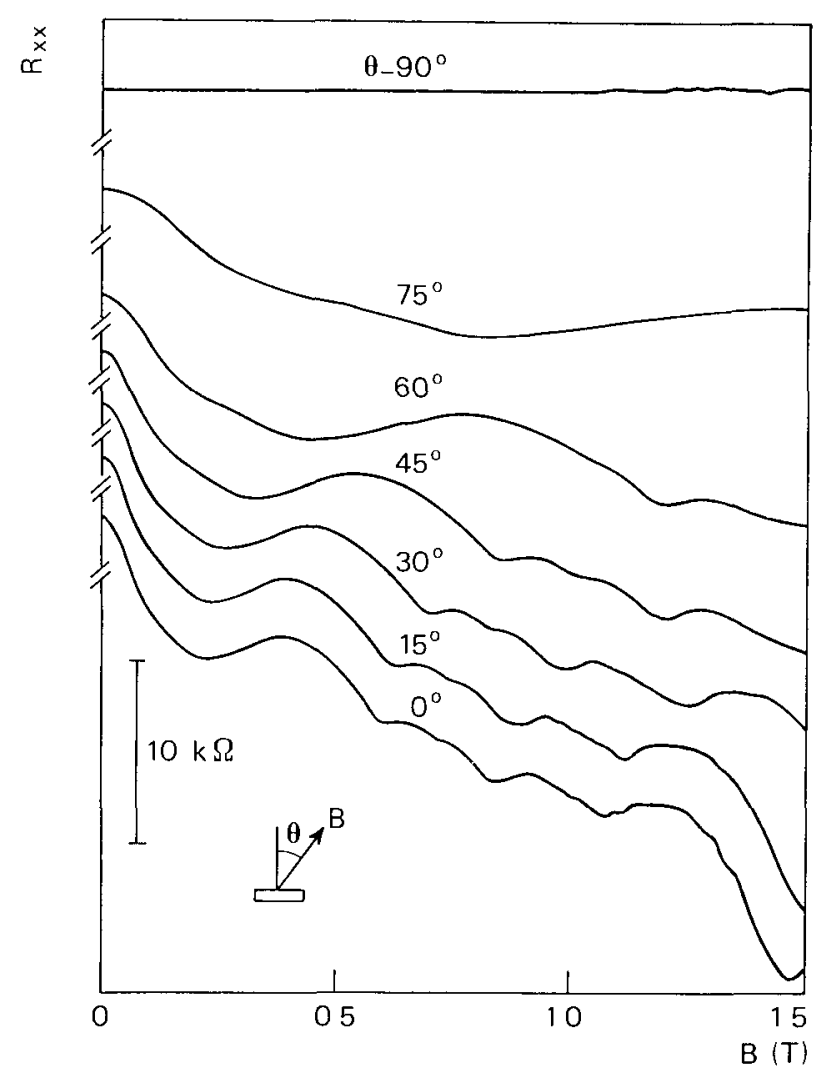

Fig 29 Angular dependence of the magnetoresistance of $\mathrm{F}_{1 \mathrm{~g}} 28$, at $4 \mathrm{~K}$, proving that it has a purely orbital origin Taken from $\mathbf{H}$ van Houten et al Superlattices and Microstructures 3, 497 (1987)

anomaly in the Shubnikov-De Haas effect is a manifestation of a quantum size effect ${ }^{167217218}$ This one figure thus summarizes the wealth of classical and quantum magnetoresistance phenomena in the quasi-ballistic transport regime

Essentially similar results were obtained by Taylor et $a^{219}$ In the field range of these experiments, ${ }^{27} 5563167219$ the magnetoresistance is exclusively caused by the enclosed flux and the Lorentz force (so called orbital effects) The Zeeman energy does not play a role This is demonstrated in Fig 29,

${ }^{217} \mathrm{~K}-\mathrm{F}$ Berggren, T J Thornton, D J Newson, and M Pepper, Phys Rev Lett 57, 1769 (1986) ${ }^{218} \mathrm{~K}-\mathrm{F}$ Berggren, G Roos, and $\mathrm{H}$ van Houten, Phys Rev B 37, 10118 (1988)

${ }^{219} \mathrm{R}$ P Taylor, P C Mdın, L Eaves, S P Bedumont, I McIntyre, S Thoms, and C D W Wilkınson, $J$ Phys Condens Matter 1, 10413 (1989) 


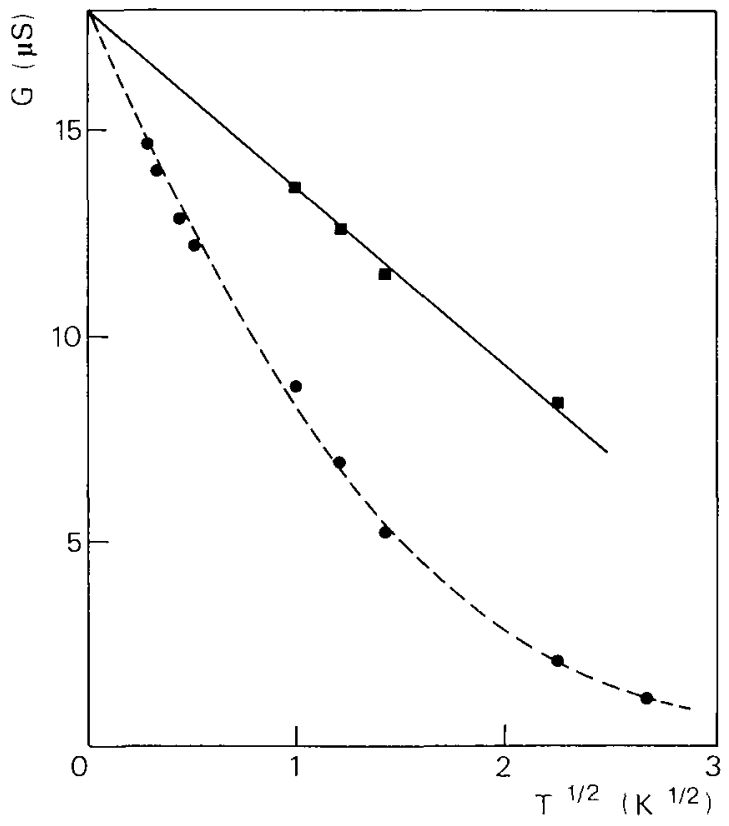

FIG 30 Zero-field conductance (circles) and conductance corrected for the weak localization effect (squares) for the channel of $F_{1 g} 28$ as a function of $T^{1 / 2}$, to demonstrate the $T^{-1 / 2}$ dependence on the temperature of the electron-electron interaction effect expected from Eq (9 1b) The solid and dashed lines are guides to the eye The extrapolated value at high temperatures is the classical part of the conductance Taken from $\mathrm{H}$ van Houten et al, Acta Electronica 28, 27 (1988)

where the magnetoresistance (obtained on the same sample as that used in Fig 28) is shown to vanish when $B$ is in the plane of the $2 D E G$ (similar results were obtained in Ref 168) In wide $2 \mathrm{DEG}$ channels a negative magnetoresistance has been found by Lin et al in a parallel magnetic field ${ }^{23} \mathrm{Th}$ is effect has been studied in deta1l by Mensz and Wheeler, ${ }^{220}$ who attributed it to a residual orbital effect associated with deviations of the 2DEG from a perfectly flat plane Fal'ko ${ }^{221}$ has calculated the effect of a magnetıc field parallel to the 2DEG on weak localization, and has found a negative magnetoresistance, but only if the scattering potential does not have reflection symmetry in the plane of the $2 \mathrm{DEG}$

In Fig 30 the temperature dependence of the zero-field conductance ${ }^{27}$ is plotted as a function of $T^{-1 / 2}$, together with the conductance after sub-

${ }^{220}$ P M Mensz, R G Wheeler, C T Foxon, and J J Harris, Appl Phys Lett 50, 603 (1987), P

M Mensz and R G Wheeler, Phys Rev B 35, 2844 (1987)

${ }^{221} \mathrm{~V}$ Falko J Phys Condens Matter 23797 (1990) 
traction of the weak localization correction The straight line through the latter data points demonstrates that the remaining temperature dependence may, indeed, be attributed to the electron-electron interactions A similar $T^{-1 / 2}$ dependence was found by Thornton et al ${ }^{58}$ in a narrow GaAsAlGaAs channel defined using the split-gate method The slope of the straight line in Fig 30 gives $g_{1 \mathrm{D}} \approx 15 \mathrm{in} \mathrm{Eq}(9 \mathrm{1b})$, which is close to the value found by Chor et al ${ }^{55}$ It should be noted, however, that this experiment is already in the regime where the quantum corrections are by no means small, so the perturbation theory is of questionable validity For this reason, and also in view of other problems (such as the difficulty in determining the effective channel width, the presence of channel width variations, and a frequently observed saturation of the weak localization correction at low temperatures due to loss of phase coherence associated with external noise or radiofrequency interference), a quantitative analysis of the parameters obtained from the weak localization and electron-electron corrections in narrow channels $\left(\tau_{\phi}\right.$ and $\left.g_{1 \mathrm{D}}\right)$ is not fully warranted Indeed, most of the narrowchannel studies available today have not been optimized for the purpose of a detailed quantitative analysis Instead, they were primarily intended for a phenomenological exploration, and as such we feel that they have been quite successful

\section{Quantum Size EfFects}

Quantum size effects on the resistivity result from modifications of the 2D density of states in a 2DEG channel of width comparable to the Fermı wavelength The electrostatic lateral confinement in such a narrow channel leads to the formation of $1 \mathrm{D}$ subbands in the conduction band of the 2DEG (see Section 4 a) The number $N \approx k_{\mathrm{F}} W / \pi$ of occupied $1 \mathrm{D}$ subbands is reduced by decreasing the Fermi energy or the channel width This depopulation of individual subbands can be detected via the resistivity An alternative method to depopulate the subbands is by means of a magnetic field perpendicular to the 2DEG The magnetic field $B$ has a negligible effect on the density of states at the Fermı level if the cyclotron diameter $2 l_{\text {cycl }} \gg W$ ( $1 \mathrm{e}$, for $B \ll B_{\text {crt }} \equiv 2 \hbar k_{\mathrm{F}} / e W$ ) If $B \gg B_{\text {crit }}$, the electrostatic confinement can be neglected for the density of states, which is then described by Landau levels [Eq (46)] The number of occupied Landau levels $N \approx E_{\mathrm{F}} / \hbar \omega_{\mathrm{c}} \approx k_{\mathrm{F}} l_{\mathrm{cycl}} / 2$ decreases linearly with $B$ for $B \gg B_{\text {crit }}$ In the intermediate field range where $B$ and $B_{\text {crit }}$ are comparable, the electrostatic confinement and the magnetic field together determine the density of states The corresponding magnetoelectric subbands are depopulated more slowly by a magnetic field than are the Landau levels, which results in an increased spacing of the Shubnikov-De Haas oscillations in the magnetoresistivity (cf Section $4 \mathrm{~d}$ )

In the following subsection we give a more quantitative description of 
magnetoelectric subbands. Experiments on the electric and magnetic depopulation of subbands in a narrow channel are reviewed in Section 9.b. We only consider here the case of a long channel $(L \gg l)$ in the quasi-ballistic regime. Quantum size effects in the fully ballistic regime $(L \lesssim l)$ are the subject of Section III.

\section{a. Magnetoelectric Subbands}

Consider first the case of an unbounded 2DEG in a perpendicular magnetic field $\mathbf{B}=\boldsymbol{\nabla} \times \mathbf{A}$. The Hamiltonian for motion in the plane of the $2 \mathrm{DEG}$ is given by

$$
\mathscr{H}=\frac{(\mathbf{p}+e \mathbf{A})^{2}}{2 m},
$$

for a single spin component. In the Landau gauge $\mathbf{A}=(0, B x, 0)$, with $\mathbf{B}$ in the $z$-direction, this may be written as

$$
\mathscr{H}=\frac{p_{x}^{2}}{2 m}+\frac{m \omega_{\mathrm{c}}^{2}}{2}\left(x-x_{0}\right)^{2}
$$

with $\omega_{\mathrm{c}} \equiv e B / m$ and $x_{0} \equiv-p_{y} / e B$. The $y$-momentum operator $p_{y} \equiv-i \hbar \partial / \partial y$ can be replaced by its eigenvalue $\hbar k_{y}$, since $p_{y}$ and $\mathscr{H}$ commute. The effect of the magnetic field is then represented by a harmonic oscillator potential in the $x$-direction, with center $x_{0}=-\hbar k_{y} / e B$ depending on the momentum in the $y$-direction. The energy eigenvalues $E_{n}=\left(n-\frac{1}{2}\right) \hbar \omega_{c}, n=1,2,3, \ldots$, do not depend on $k_{y}$ and are therefore highly degenerate. States with the same quantum number $n$ are referred to collectively as Landau levels. ${ }^{93}$ The number of Landau levels below energy $E$ is given by

$$
N=\operatorname{Int}\left[1 / 2+E / \hbar \omega_{\mathrm{c}}\right],
$$

where Int denotes truncation to an integer.

A narrow channel in the $y$-direction is defined by an electrostatic confining potential $V(x)$. The case of a parabolic confinement is easily solved analytically. ${ }^{36,218,222,223}$ Adding a term $V(x)=\frac{1}{2} m \omega_{0}^{2} x^{2}$ to the hamiltonian (10.2), one finds, after a rearrangement of terms,

$$
\mathscr{H}=\frac{p_{x}^{2}}{2 m}+\frac{m \omega^{2}}{2}\left(x-\bar{x}_{0}\right)^{2}+\frac{\hbar^{2} k^{2}}{2 M},
$$

with $\omega \equiv\left(\omega_{\mathrm{c}}^{2}+\omega_{0}^{2}\right)^{1 / 2}, \bar{x}_{0} \equiv x_{0} \omega_{\mathrm{c}} / \omega$, and $M \equiv m \omega^{2} / \omega_{0}^{2}$. The first two terms describe the motion in the $x$-direction in a harmonic potential with effective frequency $\omega \geqslant \omega_{0}$, and the third term describes free motion in the $y$-direction

${ }^{222}$ L Smrcka, H Havlova, and A Ishara, J Phys. C 19, L457 (1986)

${ }^{223}$ K -F Berggren, and D J Newson, Semicond. Sct Technol. 1, 327 (1986) 
with an effective mass $M \geqslant m$ This last term removes the degeneracy of the Landau levels, which become 1D subbands with energy

$$
E_{n}(k)=\left(n-\frac{1}{2}\right) \hbar \omega+\hbar^{2} k^{2} / 2 M
$$

The subband bottoms have energy $E_{n}=\left(n-\frac{1}{2}\right) \hbar \omega$, and the number of subbands occupied at energy $E$ is $N=\operatorname{Int}\left[\frac{1}{2}+E / \hbar \omega\right]$ The quasi-1D density of states is obtained from Eq (44) on substituting $m$ for $M$ For the comparison with experiments it is useful to define an effective width for the parabolic potential One can take the width $W_{\text {par }}$ to be the separation between the equipotentials at the Fermi energy

$$
W_{\text {par }} \equiv 2 \hbar k_{\mathrm{F}} / m \omega_{0}
$$

(An alternative, which differs only in the numerical prefactor, is to take $W_{\text {par }} \equiv n_{1 \mathrm{D}} / n_{\mathrm{s}}$, with $n_{\mathrm{s}} \equiv g_{\mathrm{s}} g_{\mathrm{v}} k_{\mathrm{F}}^{2} / 4 \pi$ the $2 \mathrm{D}$ sheet density and $n_{1 \mathrm{D}}$ the number of electrons per unit length in the narrow channel ${ }^{218}$ ) The number of occupied magnetoelectric subbands at energy $E_{\mathrm{F}}$ in a parabolic confinıng potential may then be written as

$$
N=\operatorname{Int}\left[\frac{1}{2}+\frac{1}{4} k_{\mathrm{F}} W_{\mathrm{par}}\left[1+\left(W_{\mathrm{par}} / 2 l_{\mathrm{cycl}}\right)^{2}\right]^{-1 / 2}\right],
$$

where $l_{\text {cycl }} \equiv \hbar k_{\mathrm{F}} / e B$ is the cyclotron radius at the Fermı energy For easy reference, we also give the result for the number of occupied subbands at the Fermı energy in a square-well confinement potentral of width $W$

$$
N \approx \operatorname{Int}\left[\frac{2}{\pi} \frac{E_{\mathrm{F}}}{\hbar \omega_{\mathrm{c}}}\left(\arcsin \frac{W}{2 l_{\mathrm{cycl}}}+\frac{W}{2 l_{\text {cycl }}}\left[1-\left(\frac{W}{2 l_{\mathrm{cycl}}}\right)^{2}\right]^{1 / 2}\right)\right], \quad \text { if } l_{\mathrm{cycl}}>\frac{W}{2},
$$

$$
N \approx \operatorname{Int}\left[\frac{1}{2}+\frac{E_{\mathrm{F}}}{\hbar \omega_{\mathrm{c}}}\right], \quad \text { if } l_{\mathrm{cycl}}<\frac{W}{2}
$$

(This result is derived in Section $12 \mathrm{a}$ in a semiclassical approximation The accuracy is \pm 1 ) One easily verifies that, for $B \ll B_{\text {crit }} \equiv 2 \hbar k_{\mathrm{F}} / \mathrm{eW}, \mathrm{Eq}$ (10 8) yields $N \approx k_{\mathrm{F}} W / \pi$ The parabolic confining potential gives $N \approx k_{\mathrm{F}} W_{\mathrm{par}} / 4 \mathrm{in}$ the weak-field limit In the strong-field limit $B \gg B_{\text {crit }}$, both potentials give the result $N \approx E_{\mathrm{F}} / \hbar \omega_{\mathrm{c}}=k_{\mathrm{F}} l_{\text {cycl }} / 2$ expected for pure Landau levels In Fig 31 we compare the depopulation of Landau levels in an unbounded 2DEG with its characteristic $1 / B$ dependence of $N$ (dashed curve), with the slower depopulation of magnetoelectric subbands in a narrow channel The dashdotted curve is for a parabolic confining potential, the solid curve for a square-well potential These results are calculated from Eqs (10 7) and (10 8), with $k_{\mathrm{F}} W_{\mathrm{par}} / \pi=k_{\mathrm{F}} W / \pi=10 \mathrm{~A} B$-independent Fermi energy was assumed in Fig 31 so that the density $n_{1 \mathrm{D}}$ oscillates around its zero-field value (For a long channel, it is more appropriate to assume that $n_{1 \mathrm{D}}$ is $B$-independent, to 


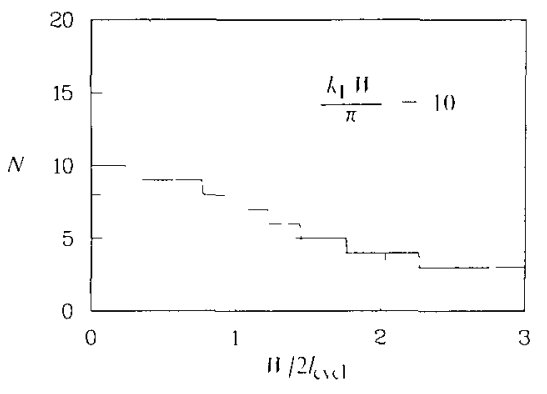

FiG. 31 Magnetic field dependence of the number $N$ of occupied subbands in a narrow channel for a parabolic confining potentral according to Eq. (10.7) (dot-dashed curve), and for a square-well confining potential according to Eq. (10.8) (full curve). The dashed curve gives the magnetıc depopulation of Landau levels in a wide $2 D E G$, which has a $1 / B$ dependence. The calculations are done for a fixed Fermi energy and for channel width $W=W_{\mathrm{par}}=10 \pi / k_{\Gamma}$

preserve charge neutrality, in which case $E_{\mathrm{F}}$ oscillates. This case is studied in Ref. 218.) Qualitatively, the two confining potentials give similar results. The numerical differences reflect the uncertainty in assigning an effective width to the parabolic potential. Self-consistent solutions of the Poisson and Schrödinger equations ${ }^{42,60,61,72,224}$ for channels defined by a split gate have shown that a parabolic potential with a flat bottom section is a more realistic model. The subband depopulation for this potential has been studied in a semiclassical approximation in Ref. 223. A disadvantage of this more realistic model is that an additional parameter is needed for its specification (the width of the flat section). For this practical reason, the use of either a parabolic or a square-well potential has been preferred in the analysis of most experiments.

\section{b. Experiments on Electric and Magnetic Depopulation of Subbands}

The observation of $1 \mathrm{D}$ subband effects unobscured by thermal smearing requires low temperatures, such that $4 k_{\mathrm{B}} T \ll \Delta E$, with $\Delta E$ the energy difference between subband bottoms near the Fermi level $\left(4 k_{\mathrm{B}} T\right.$ being the width of the energy averaging function $d f / d E_{\mathrm{F}}$; see Section 4.b; For a square well $\Delta E \approx 2 E_{\mathrm{F}} / N$, and for parabolic confinement $\Delta E \approx E_{\mathrm{F}} / N$ ). Moreover, the formation of subbands requires the effective mean free path (limited by impurity scattering and diffuse boundary scattering) to be much larger than $W$ (cf. also Ref. 218). The requirement on the temperature is not difficult to meet, $\Delta E / 4 k_{\mathrm{B}} T$ being on the order of $50 \mathrm{~K}$ for a typical GaAs-AlGaAs channel of width $W=100 \mathrm{~nm}$, and the regime $l>W$ is also well accessible. These simple considerations seem to suggest that $1 \mathrm{D}$ subband effects should be rather easily observed in semiconductor nanostructures. This conclusion is misleading, however, and in reality manifestations of $1 \mathrm{D}$ subband structure have been elusive, at least in the quasi-ballistic regime $W<l<L$. The main reason for this is the appearance of large conductance fluctuations that mask the subband structure if the channel is not short compared with the mean free path.

${ }^{224}$ J. A. Brum and G. Bastard, Superlattices and Microstructures 4, 443 (1988). 
Calculations ${ }^{225-227}$ of the average conductivity of an ensemble of narrow channels do in fact show oscillations from the electric depopulation of subbands [resultıng from the modulation of the density of states at the Fermı level, which determınes the scatterıng tıme, see Eq (4 28)] The oscillations are not as large as the Shubnikov-De Haas oscillations from the magnetic depopulation of Landau levels or magnetoelectric subbands One reason for this difference is that the peaks in the density of states become narrower, relative to their separation, on applying a magnetic field (The quantum limit of a single occupied 1D subband has been studied in Refs 42 and 228-230)

In an individual channel, aperiodic conductance fluctuations due to quantum interference (see Section 7) are the dominant cause of structure in the low-temperature conductance as a function of gate voltage (which corresponds to a variation of the Fermi energy), as was found in experiments on narrow $S_{1}$ inversion layers ${ }^{46161162}$ Warren et al ${ }^{44}$ were able to suppress these fluctuations by performing measurements on an array of narrow channels in a $S_{1}$ inversion layer In Fig 32 we reproduce their results The structure due to the electric depopulation of $1 \mathrm{D}$ subbands is very weak in the current-versus-gate-voltage plot, but a convincingly regular oscillation is seen if the derivative of the current with respect to the gate voltage is taken (this quantity is called the transconductance) Warren et al pointed out that the observation of a quantum size effect in an array of 250 channels indicates a rather remarkable uniformity of the width and density of the individual channels

More recently a sımular experimental study was performed by Ismail et $a l^{231}$ on 100 parallel channels defined in the 2DEG of a GaAs-AlGaAs heterostructure The effects were found to be much more pronounced than in the earlier experiment on Si inversion layer channels, presumably because of the much larger mean free path (estimated at $1 \mu \mathrm{m}$ ), which was not much shorter than the sample length $(5 \mu \mathrm{m})$ Quantum size effects in the quantum ballistic transport regime (in particular, the conductance quantization of a quantum point contact) are discussed extensively in Section 13

In a wide 2DEG the minıma of the Shubnikov-De Haas oscillations in the magnetoresistance are periodic in $1 / B$, with a periodicity $\Delta(1 / B)$ determined by the sheet density $n_{\mathrm{s}}$ according to Eq (429) In a narrow channel one

${ }^{225} \mathrm{M}$ J Kearney and P N Butcher, $J$ Phys C 20, 47 (1987)

${ }^{226} \mathrm{~S}$ Das Sarmd and X C Xie, Phys Rev B 35, 9875 (1987)

${ }^{227} \mathrm{P}$ Vasilopoulos and F M Peeters, Phys Rev B 40, 10079 (1989)

${ }^{228}$ H Sakak1, Jap J Appl Phys 19, L735 (1980)

${ }^{229} \mathrm{G}$ Fishman, Phys Rev B 36, 7448 (1987)

${ }^{230} \mathrm{~J}$ Lee and M O Vassell, $J$ Phys C 17, 2525(1984), J Lee and H N Spector, J Appl Phys 57 $366(1985)$

${ }^{231} \mathrm{~K}$ Ismanl, D A Antoniadis, and H I Smith, Appl Phys Lett 54, 1130 (1989) 
a

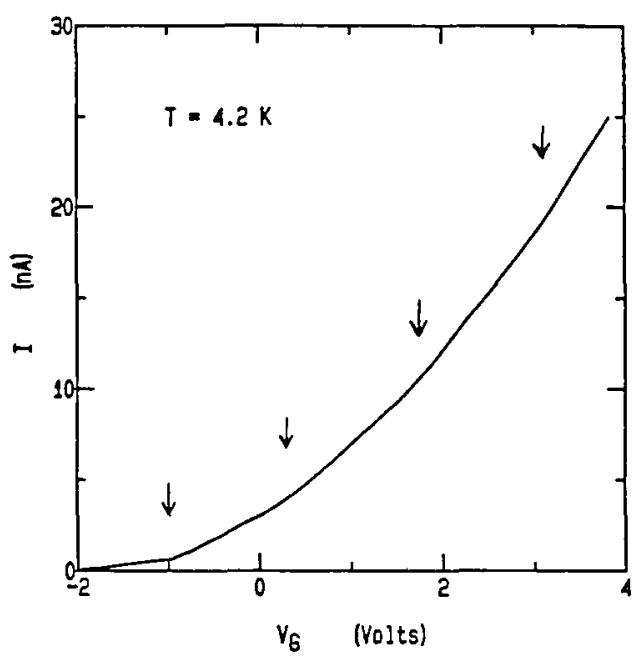

b

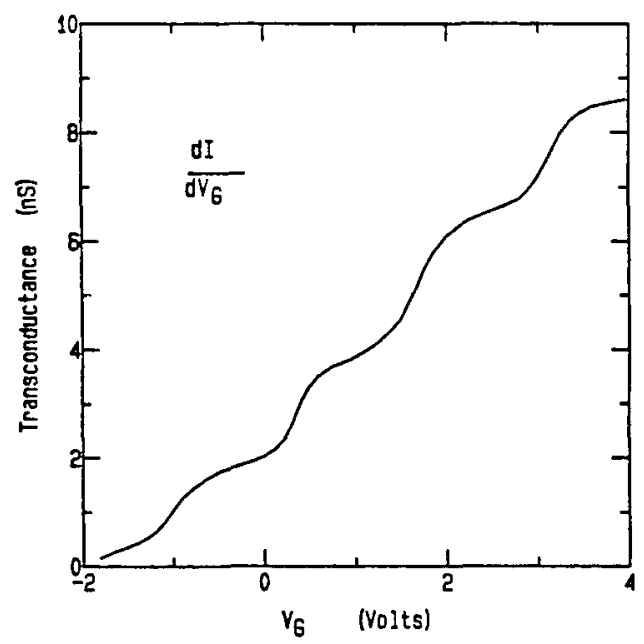

Fig 32 (a) Dependence on the gate voltage of the current $I$ through 250 parallel narrow $S 1$ inversion layer channels at $12 \mathrm{~K}$, showing the electric depopulation of subbands (b) The effect is seen more clearly in the transconductance $d I / d V_{\mathrm{G}}$ Note the absence of universal conductance fluctuations, which have been averaged out by the large number of channels Taken from A $\mathrm{C}$ Warren et al, IEEE Electron Device Lett EDL-7, 413 (1986) 
observes an increase in $\Delta(1 / B)$ for weak magnetic fields because the electrostatic confinement modifies the density of states, as discussed in Section 10 a Such a deviation is of interest as a manifestation of magnetoelectric subbands, but also because it can be used to estimate the effective channel width using the criterion $W \approx 2 l_{\text {cycl }}$ for the crossover field ${ }^{167} B_{\text {crit }}$ (the electron density in the channel, and hence $l_{\text {cycl }}$, may be estimated from the strong-field periodicity) The phenomenon has been studied in many publications 3656577479167217218223232233

As an illustration, we reproduce in Fig 33a an experimental magnetoresistance trace ${ }^{167218}$ obtained for a narrow $(W \approx 140 \mathrm{~nm}) \mathrm{GaAs}-$ AlGaAs channel, defined using a shallow-mesa etch ${ }^{63}$ The arrows indicate the magnetoresistance minima thought to be associated with magnetic depopulation The assignment becomes ambiguous in weak magnetic fields, because of the presence of aperiodic conductance fluctuations Nevertheless, the deviation from a straight line in the $N$ versus $B^{-1}$ plot in Fig 33b is sufficiently large to be reasonably convincing Also shown in Fig $33 \mathrm{~b}$ is the result of a fit to a theoretical $N(B)$ function (assuming a parabolic confining potential and a $B$-independent electron density) The parameter values found from this fit for the width and electron density are reasonable and agree with independent estimates ${ }^{27}$

We have limited ourselves to a discussion of transport studies, but wish to point out that 1D subbands have been studied succesfully by capacitance ${ }^{75}$ measurements and by infrared ${ }^{78}$ spectroscopy As mentioned earlier, the formation of 1D subbands also requires a reformulation of the theories of weak localization and conductance fluctuations in the presence of boundary scattering Weak localization in the case of a small number of occupied subbands has been studied by Tesanovic et al ${ }^{110234}$ (1n a zero magnetic field)

We will not discuss the subject of quantum size effects further in this part of our review, since it has found more striking manifestations in the ballistic transport regime (the subject of Section III), where conductance fluctuations do not play a role The most prominent example is the conductance quantization of a point contact

\section{Periodic Potential}

\section{a Lateral Superlattices}

In a crystal, the periodic potential of the lattice opens energy gaps of zero density of electronic states An electron with energy in a gap is Bragg-

\footnotetext{
${ }^{232}$ M Lakrimı, A D C Grassie, K M Hutchings, J J Harris, and C T Foxon, Semicond Scl

Technol 4, 313 (1989)

${ }^{233}$ J J Alsmeier, Ch Sikorsk1, and U Merkt, Phys Rev B 37, 4314 (1988)

${ }^{234}$ Z Tesanovic, $J$ Phys C 20, L829 (1987)
} 

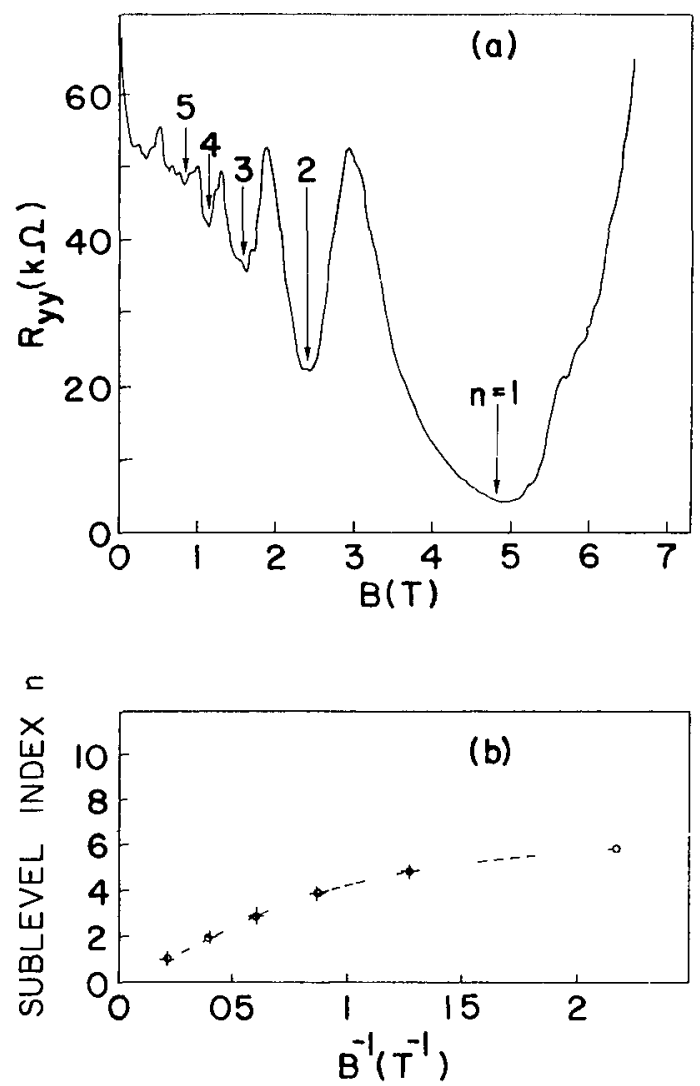

FIG 33 (a) Magnetoresistance at $24 \mathrm{~K}$ of a narrow GaAs-AlGaAs channel (as in Fig 28) The arrows indicate magnetic field values assigned to the depopulation of magnetoelectric subbands (b) Subband index $n \equiv N-1$ versus inverse magnetic field (crosses) The dashed line interpolates between theoretical points for a parabolic confining potential (circles) The electrostatic confinement causes deviations from a linear dependence of $n$ on $B^{-1}$ Taken from K -F Berggren et al, Phys Rev B 37, 10118 (1988)

reflected and hence cannot propagate through the crystal Esak1 and Tsu ${ }^{235}$ proposed in 1970 that an artificial energy gap might be created by the epitaxial growth of alternating layers of different semiconductors In such a superlattice a periodic potential of spacing $a$ is superimposed on the crystal lattice potential Typically, $a \approx 10 \mathrm{~nm}$ is chosen to be much larger than the crystal lattice spacing $(05 \mathrm{~nm})$, leading to the formation of a large number of narrow bands within the conduction band (minibands), separated by small energy gaps (minigaps) Qualitatıvely new transport properties may then be expected For example, the presence of minigaps may be revealed under 
strong applied voltages by a negative differential resistance-a phenomenon predicted by Esak1 and Tsu in their original proposal and observed subsequently by Esak1 and Chang ${ }^{236237}$ In contrast to a 3D crystal lattice, a superlattıce formed by alternatıng layers is $1 \mathrm{D}$ As a consequence of the free motion in the plane of the layers, the density of states is not zero in the minigaps, and electrons may scatter between two overlapping minibands of interest in the present context is the possibility of defining lateral superlattices $^{238} 239$ by a periodic potential in the plane of a $2 \mathrm{D}$ electron gas True minigaps of zero density of states may form in such a system if the potential varies periodically in two directions Lateral superlattice effects may be studied in the linear-response regime of small applied voltages (to which we limit the discussion here) by varying $E_{\mathrm{F}}$ or the strength of the periodic potential by means of a gate voltage The conductivity is expected to vanish if $E_{\mathrm{F}}$ is in a true minigap (so that electrons are Bragg-reflected) Calculations $^{240241}$ show pronounced minima also in the case of a 1D periodic potential

The conditions required to observe the minibands in a lateral superlattice are similar to those discussed in Section 10 for the observation of 1D subbands in a narrow channel The mean free path should be larger than the lattice constant $a$, and $4 k_{\mathrm{B}} T$ should be less than the width of a minigap near the Fermi level For a weak periodic potentral, ${ }^{94}$ the $n$th minigap is approximately $\Delta E_{n} \approx 2 V_{n}$, with $V_{n}$ the amplitude of the Fourier component of the potential at wave number $k_{n}=2 \pi n / a$ The gap is centered at energy $E_{n} \approx\left(\hbar k_{n} / 2\right)^{2} / 2 m$ If we consider, for example, a 1D sinusoidal potential $V(x, y)=V_{0} \sin (2 \pi y / a)$, then the first energy gap $\Delta E_{1} \approx V_{0}$ occurs at $E_{1} \approx(\hbar \pi / a)^{2} / 2 m$ (Higher-order minigaps are much smaller) Bragg reflection occurs when $E_{1} \approx E_{\mathrm{F}}\left(1 \mathrm{e}\right.$, for a lattice periodicity $\left.a \approx \lambda_{\mathrm{F}} / 2\right)$ Such a shortperiod modulation is not easy to achieve lithographically, however (typically $\lambda_{\mathrm{F}}=40 \mathrm{~nm}$ ), and the experiments on lateral superlattices discussed later are not in this regime

Warren et $a l^{242}$ have observed a weak but regular structure in the conductance of a $1 \mathrm{D}$ lateral superlattice with $a=02 \mu \mathrm{m}$ defined in a $\mathrm{S}_{1}$ inversion layer (using the dual-gate arrangement of Fig 2c) Ismail et al ${ }^{62}$ used a grating-shaped gate on top of a GaAs-AlGaAs heterostructure to

${ }^{236} \mathrm{~L}$ Esak1 and L L Chang, Phys Rev Lett 33, 495 (1974)

${ }^{237}$ L Esak1, Rev Mod Phys 46, 237 (1974)

${ }^{238} \mathrm{H}$ Sakak1, K Wagatsuma, J Hamasak1, and S Satto, Thin Soldd Films 36, 497 (1976)

${ }^{239} \mathrm{R}$ T Bate, Bull Am Phys Soc 22, 407 (1977)

${ }^{240}$ M J Kelly, J Phys C 18, 6341 (1985), Surf Scl 170, 49 (1986)

${ }^{241}$ P F Bagwell and T P Orlando, Phys Rev B 40, 3735 (1989)

${ }^{242}$ A C Warren, D A Antoniadıs, H I Smith, and J Melngailıs, IEEE Electron Device Letts, EDL-6, 294 (1985) 


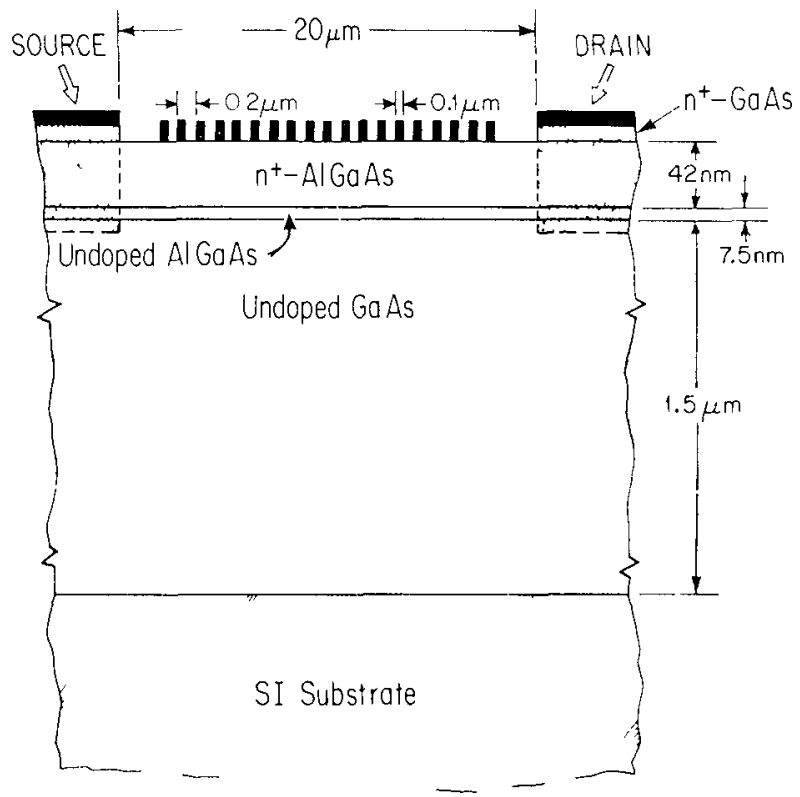

FIG. 34. Gratıng gate (in black) on top of a GaAs-AIGaAs heterostructure, used to define a 2DEG with a periodic density modulation. Taken from K. Ismail et al., Appl. Phys. Lett. 52, 1071 (1988).

define a lateral superlattice. A schematic cross section of their device is shown in Fig. 34. The period of the grating is $0.2 \mu \mathrm{m}$. One effect of the gate voltage is to change the overall carrier concentration, leading to a large but essentially smooth conductance variation (at $4.2 \mathrm{~K}$ ). This variation proved to be essentially the same as that found for a continuous gate. As in the experiment by Warren et al., the transconductance as a function of the voltage on the grating revealed a regular oscillation. As an example, we reproduce the results of Ismail et al. (for various source-drain voltages) in Fig. 35. No such structure was found for control devices with a continuous, rather than a grating, gate. The observed structure is attributed to Bragg reflection in Ref. 62. A 2D lateral superlattice was defined by Bernstein and Ferry, ${ }^{243}$ using a grid-shaped gate, but the transport properties in the linear response regime were not studied in detail. Smith et al. ${ }^{244}$ have used the split-gate technique to define a $2 \mathrm{D}$ array of 4000 dots in a high-mobility GaAs-AlGaAs heterostructure $(a=0.5 \mu \mathrm{m}, l=10 \mu \mathrm{m})$. When the 2DEG under the dots is depleted,

${ }^{243}$ G. Bernstein and D. K. Ferry, J. Vac. Sci. Technol. B 5, 964 (1987).

${ }^{244}$ C. G. Smith, M. Pepper, R. Newbury, H. Ahmed, D. G. Hasko, D. C. Peacock, J. E. F. Frost, D. A. Ritchie, and G. A. C. Jones, J. Phys. Condens. Matter 2, 3405 (1990). 


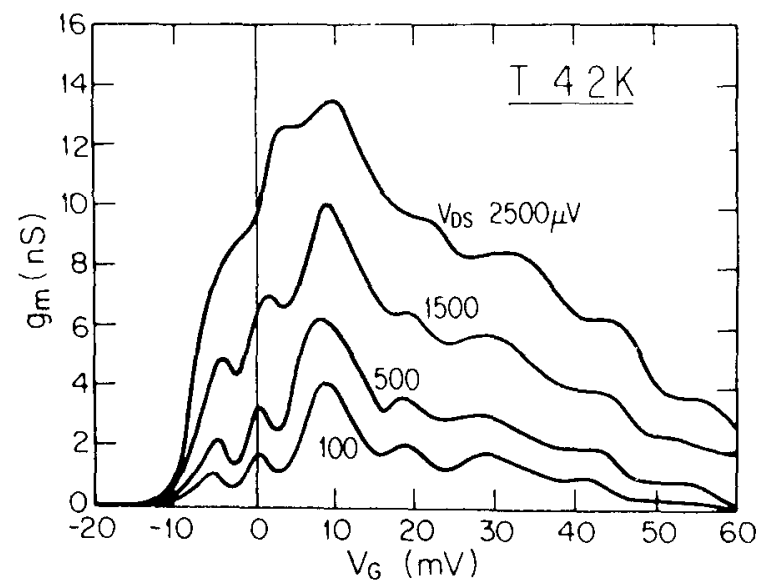

FIG 35 Transconductance $g_{\mathrm{m}} \equiv \partial I / \partial V_{\mathrm{SD}}$ of the device of Fig 34 measured as a function of gate voltage for various values of the source-drain voltage The oscillations, seen in particular at low source-drain voltages, are attributed to Bragg reflection in a periodic potential Taken from K Ismall et al, Appl Phys Lett 5a, 1071 (1988)

a grid of conducting channels is formed In this experiment the amplitude of the periodic potential exceeds $E_{\mathrm{F}}$ Structure in the conductance is found related to the depopulation of $1 \mathrm{D}$ subbands in the channels, as well as to standing waves between the dots The analysis is thus considerably more complicated than it would be for a weak periodic potential It becomes difficult to distinguish between the effects due to quantum interference within a single unit cell of the perrodic potential and the effects due to the formation of minibands requiring phase coherence over several unit cells Devices with a 2D periodic potential with a period comparable to the Fermı wavelength and much shorter than the mean free path will be required for the realization of true miniband effects It appears that the fabrication of such devices will have to awat further developments in the art of making nanostructures Epitaxy on tilted surfaces with a staircase surface structure is being investigated for this purpose 8788169179245246 Nonepitaxial growth on Si surfaces slightly tilted from (100) is known to lead to miniband formation in the inversion layer ${ }^{20247} \mathrm{~A}$ final interesting possibility is to use doping quantum wires, as proposed in Ref 248

As mentioned, it is rather difficult to discriminate experimentally between true minıband effects and quantum interference effects occurring within one

${ }^{245}$ J M Gaines, P M Petroff, H Kroemer, R J Simes, R S Geels, and J H English, $J$ Vac Scl

Technol B 61378 (1988)

${ }^{246}$ H Sakak1, Jap J Appl Phys 28, L314 (1989)

${ }^{247}$ T Cole, A A Lakhan1, and P J Strles, Phys Rev Lett 38, 722 (1977)

${ }^{248} \mathrm{G}$ E W Bauer and A A van Gorkum, in Ref 16 
unit cell The reason is that both phenomena give rise to structure in the conductance as a function of gate voltage with essentially the same periodicity This difficulty may be curcumvented by studying lateral superlattices with a small number of unit cells The miniband for a finite superlattice with $P$ unit cells consists of a group of $P$ discrete states, which merge into a continuous miniband in the limit $P \rightarrow \infty$ The discrete states give rise to closely spaced resonances in the transmission probability through the superlattice as a function of energy, and may thus be observed as a series of $P$ peaks in the conductance as a function of gate voltage, separated by broad minima due to the minıgaps Such an observation would demonstrate phase coherence over the entire length $L=P a$ of the finite superlattice and would constitute conclusive evidence of a miniband The conductance of a finite $1 \mathrm{D}$ superlattice in a narrow 2DEG channel in the ballistic transport regime has been investigated theoretically by Ulloa et al ${ }^{249}$ Similar physics may be studied in the quantum Hall effect regime, where the experimental requirements are considerably relaxed A successful experiment of this type was recently performed by Kouwenhoven et al ${ }^{250}$ (see Section 22)

Weak-field magnetotransport in a 2D periodic potential (a grid) has been studied by Ferry et $a l^{251252}$ and by Smith et al ${ }^{244}$ Both groups reported oscillatory structure in the magnetoconductance, suggestive of an Aharonov-Bohm effect with periodicity $\Delta B=h / e S$, where $S$ is the area of a unit cell of the "lattice" In strong magnetic fields no such oscillations are found A simular suppression of the Aharonov-Bohm effect in strong fields is found in single rings, as discussed in detall in Section 21 a Magnetotransport in a $1 \mathrm{D}$ periodic potential is the subject of the next subsection

\section{b Gulding-Center-Drift Resonance}

The influence of a magnetic field on transport through layered superlattices ${ }^{253}$ has been studied mainly in the regime where the (first) energy gap $\Delta E \sim 100 \mathrm{meV}$ exceeds the Landau level spacing $\hbar \omega_{\mathrm{c}}(17 \mathrm{meV} / \mathrm{T}$ in $\mathrm{GaAs})$ The magnetic field does not easily induce transitions between different minıbands in this regime Magnetotransport through lateral superlattices is often in the opposite regime $\hbar \omega_{\mathrm{c}} \gg \Delta E$, because of the relatively large periodicity $(a \sim 300 \mathrm{~nm})$ and small amplitude $\left(V_{0} \sim 1 \mathrm{meV}\right)$ of the periodic potentral The magnetıc field now changes qualitatıvely the structure of the

\footnotetext{
${ }^{249}$ S E Ulloa, E Castano, and G Kirczenow, Phys Rev B 41, 12350 (1990)

${ }^{250}$ L P Kouwenhoven, F W J Hekkıng, B J van Wees, C J P M Harmans, C E Timmerıng, and C T Foxon, Phys Rev Lett 65, 361 (1990)

${ }^{251} \mathrm{D}$ K Ferry, in Ref 14

${ }^{252}$ P A Puechner, J Md, R Mezenner, W -P Liu, A M Krıman, G N Maracas, G Bernsteın, and D K Ferry, Surf $S_{C l}$ 27, 137 (1987)

${ }^{253} \mathrm{~J}$ C Madn, Festkorperprobleme 27, 137 (1987)
} 

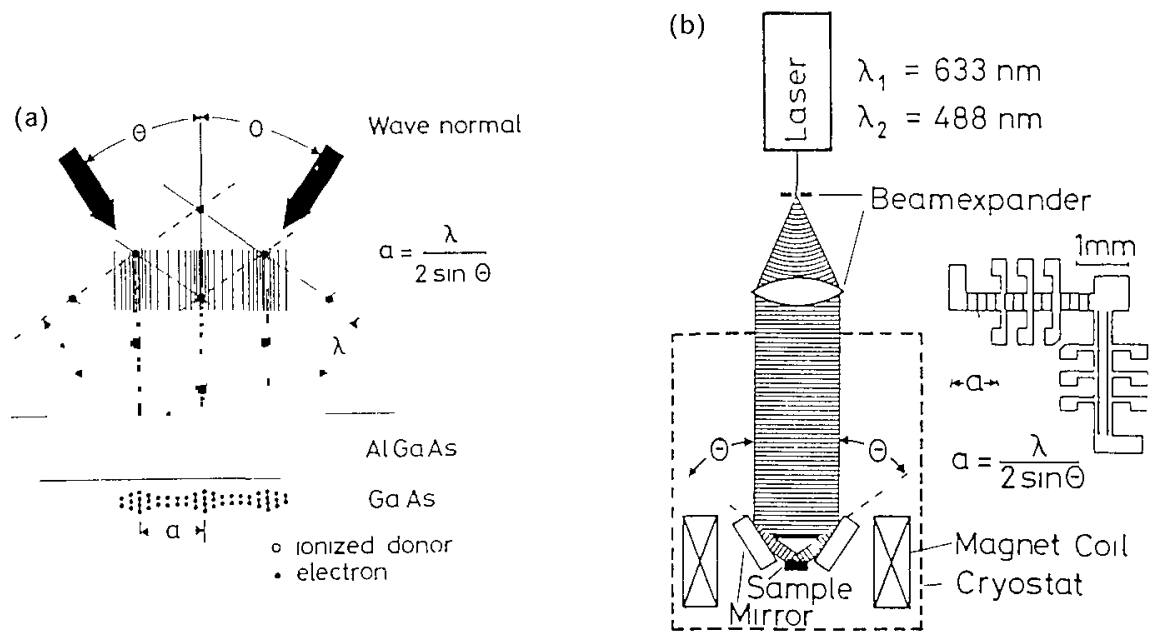

Fig 36 (a) A brief illumination of a GaAs-AlGaAs heterostructure with an interference pattern due to two laser beams (black arrows) leads to a persistent periodic variation in the concentration of ionized donors in the AlGaAs, thereby imposing a weak periodic potential on the 2DEG The resulting spatial variation of the electron density in the 2DEG is indicated schematically (b) Experımental arrangement used to produce a modulated 2DEG by means of the "holographic illumination" of (a) The sample layout shown allows measurements of the resistivity parallel and perpendicular to the equipotentidls Taken from D Weiss et al, in "High Magnetic Fields in Semiconductor Physics II" (G Landwehr, ed) Springer, Berlin, 1989

energy bands, which becomes richly complex in the case of a $2 \mathrm{D}$ periodic potentral. $^{254}$ Much of this structure, however, is not easily observed, and the experıments discussed in this subsection involve mostly the classical effect of a weak periodic potential on motion in a magnetic field.

Weiss et al. ${ }^{255,256}$ used an ingenious technique to impose a weak periodic potentral on a $2 \mathrm{DEG}$ in a GaAs-AlGaAs heterostructure. They exploit the well-known persistent ionization of donors in AlGaAs after brief illumination at low temperatures. For the illumination, two interfering laser beams are used, which generate an interference pattern with a period depending on the wavelength and on the angle of incidence of the two beams. This technique, known as holographic illumination, is illustrated in Fig. 37. The interference pattern selectıvely ionızes $S_{1}$ donors in the $\mathrm{AlGaAs}$, leading to a weak

${ }^{254}$ D R Hofstadter, Phys Rev B 14, 2239 (1976)

${ }^{255}$ D Weiss, K von Klitzing, K Ploog, and G Weimann, Europhys Lett 8, 179 (1989), also in "High Magnetic Fields in Semiconductor Physıcs II" (G Landwehr, ed) Springer, Berlın, 1989

${ }^{256} \mathrm{D}$ Weiss, C Zhang, R R Gerhardts, $\mathrm{K}$ von Klıtzıng, and G Weimann, Phys Rev B 39, 13020 (1989) 


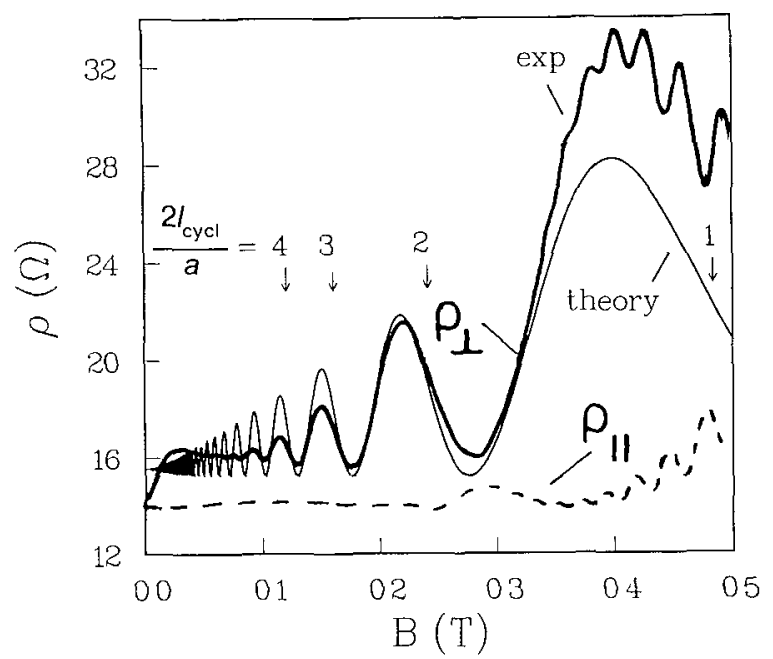

Fig 37 Solud curves Magnetic field dependence of the resistivity $\rho_{\perp}$ for current flowing perpendicular to a potential grating The experimental curve is the measurement of Weiss et $a l,{ }^{255}$ the theoretical curve follows from the guiding-center-drift resonance Note the phase shift of the oscillations, indicated by the arrows at integer $2 l_{\text {cycl }} / a$ The potential gratıng has periodicity $a=382 \mathrm{~nm}$ and is modeled by a sinusoidal potential with root-mean-square amplitude of $\varepsilon=15 \%$ of the Fermi energy, The mean free path in the 2DEG is $12 \mu \mathrm{m}$, much larger than $a$ The dash-dotted curve is the experimental resistivity $\rho_{\|}$for current flowing parallel to the potential gratıng, as measured by Weiss et al Taken from C W J Beenakker, Phys Rev Lett 62, 2020 (1989)

periodic modulation $V(y)$ of the bottom of the conduction band in the 2DEG, which persists at low temperatures if the sample is kept in the dark. The sample layout, also shown in Fig. 36, allows independent measurements of the resistivity $\rho_{y y}\left(\equiv \rho_{\perp}\right)$, perpendicular to, and $\rho_{x x}\left(\equiv \rho_{\|}\right)$parallel to the gratıng. In Fig. 37 we show experimental results of Weiss et al. ${ }^{255}$ for the magnetoresistivity of a 1D lateral superlattice $(a=382 \mathrm{~nm})$. In a zero magnetic field, the resistivity tensor $\rho$ is approximately isotropic: $\rho_{\perp}$ and $\rho_{i \mid}$ are indistinguishable experımentally (see Fig. 37). This indicates that the amplitude of $V(y)$ is much smaller than the Fermi energy $E_{\mathrm{F}}=11 \mathrm{meV}$. On application of a small magnetic field $B(\lesssim 0.4 \mathrm{~T})$ perpendicular to the $2 \mathrm{DEG}$, a large oscillation periodic in $1 / B$ develops in the resistivity $\rho_{\perp}$ for current flowing perpendicular to the potential grating. The resistivity is now strongly anisotropic, showing only weak oscillations in $\rho_{\|}$(current parallel to the potential gratıng). In appearance, the oscillations resemble the ShubnikovDe Haas oscillations at higher fields, but their different periodicity and much weaker temperature dependence point to a different origin.

The effect was not anticipated theoretically, but now a fairly complete and consistent theoretical picture has emerged from several ana- 
lyses $111227257-259$ The strong oscillations in $\rho_{\perp}$ result from a resonance ${ }^{111}$ between the periodic cyclotron orbit motion and the oscillating $\mathbf{E} \times \mathbf{B}$ drift of the orbit center induced by the electric field $\mathbf{E} \equiv-\nabla V$ Such guiding-centerdrift resonances are known from plasma physics, ${ }^{260}$ and the experiment by Weiss et al appears to be the first observation of this phenomenon in the solıd state Magnetic quantization is not essential for these strong oscillations, but plays a role in the transition to the Shubnikov-De Haas oscillations at higher fields and in the weak oscillations in $\rho_{\|} 227259 \mathrm{~A}$ simplified physical picture of the guiding-center-drift resonance can be obtained as follows ${ }^{111}$

The guiding center $(X, Y)$ of an electron at position $(x, y)$ having velocity $\left(v_{x}, v_{y}\right)$ is given by $X=x-v_{y} / \omega_{c}, Y=y+v_{x} / \omega_{\mathrm{c}}$ The time derivative of the guiding center is $X=E(y) / B, Y=0$, so its motion is parallel to the $x$-axis This is the $E \times B$ drift In the case of a strong magnetic field and a slowly varying potential $\left(l_{\text {cycl }} \ll a\right)$, one may approximate $E(y) \approx E(Y)$ to close the equations for $\dot{X}$ and $\dot{Y}$ This so-called adiabatic approximation cannot be made in the weak-field regime $\left(l_{\text {cycl }} \gtrsim a\right)$ of interest here We consider the case of a weak potential, such that $e V_{\text {rms }} / E_{\mathrm{F}} \equiv \varepsilon \ll 1$, with $V_{\text {rms }}$ the root mean square of $V(y)$ The guiding center drift in the $x$-direction is then simply superimposed on the unperturbed cyclotron motion Its time average $v_{\text {drift }}$ is obtained by integrating the electric field along the orbit

$$
v_{\mathrm{drift}}(Y)=(2 \pi B)^{-1} \int_{0}^{2 \pi} d \phi E\left(Y+l_{\text {cycl }} \sin \phi\right)
$$

For $l_{\text {cycl }} \gg a$ the field oscillates rapidly, so only the drift acquired close to the two extremal points $Y \pm l_{\text {cycl }}$ does not average out It follows that $v_{\text {drift }}$ is large or small depending on whether $E\left(Y+l_{\text {cycl }}\right)$ and $E\left(Y-l_{\text {cycl }}\right)$ have the same sign or opposite sign (see Fig 38) For a sinusoidal potential $V(y)=\sqrt{2} V_{\mathrm{rms}} \sin (2 \pi y / a)$, one easily calculates by averaging over $Y$ that, for $l_{\text {cycl }} \gg a$, the mean square drift is

$$
\left\langle v_{\mathrm{drIft}}^{2}\right\rangle=\left(v_{\mathrm{F}} \varepsilon\right)^{2}\left(\frac{l_{\mathrm{cycl}}}{a}\right) \cos ^{2}\left(\frac{2 \pi l_{\mathrm{cycl}}}{a}-\frac{\pi}{4}\right)
$$

The guiding center drift by itself leads, for $l_{\text {cycl }} \ll l$, to $1 \mathrm{D}$ diffusion with diffusion coefficient $\delta D$ given by

$$
\delta D=\int_{0}^{\infty}\left\langle v_{\mathrm{drft}}^{2}\right\rangle \mathrm{e}^{t / \tau} d t=\tau\left\langle v_{\mathrm{drff}}^{2}\right\rangle
$$

${ }^{257} \mathrm{R}$ R Gerhardts, D Weiss, and K von Klitzing, Phys Rev Lett 62, 1173 (1989)

${ }^{258} \mathrm{R}$ W Winkler, J P Kotthaus, and K Ploog, Phys Rev Lett 62, 1177 (1989)

${ }^{259} \mathrm{P}$ Vasilopoulos and F M Peeters, Phys Rev Lett 63, 2120 (1989), R R Gerhardts and C

Zhang, Phys Rev Lett 64, 1473 (1990) 

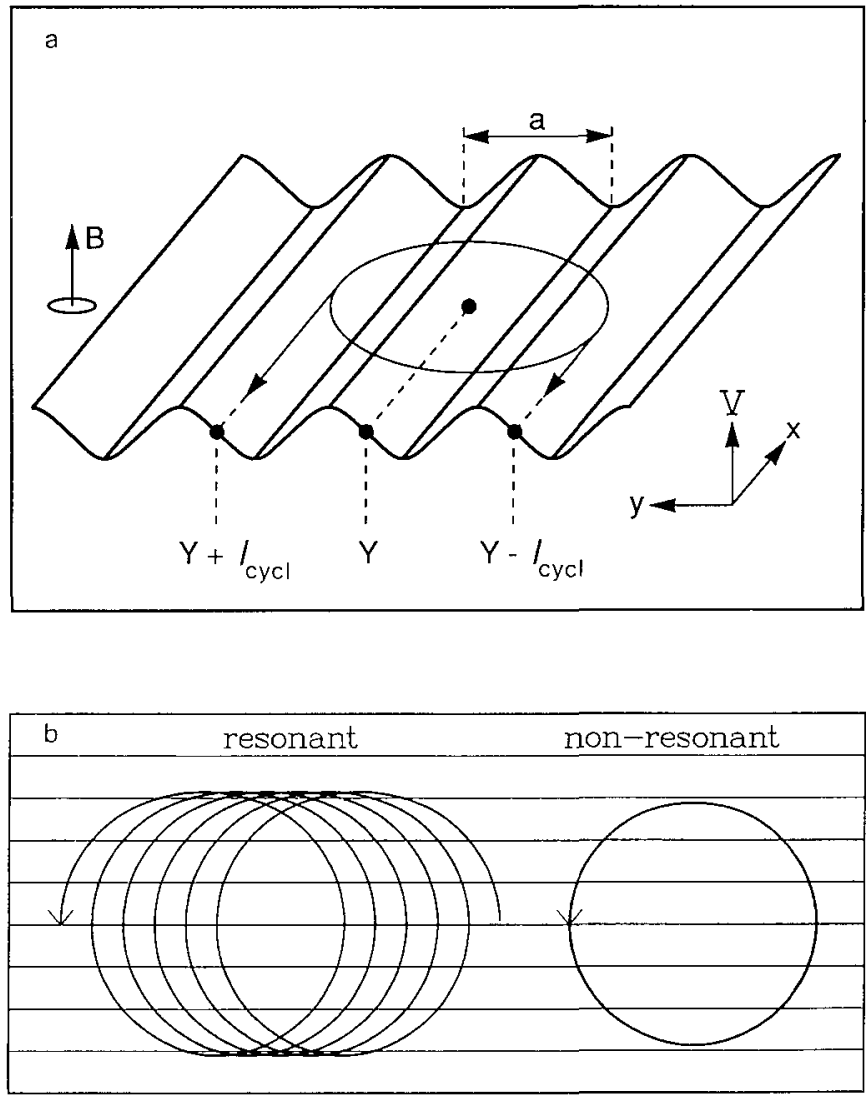

FIG 38 (a) Potential gratıng with a cyclotron orbit superimposed When the electron is close to the two extremal points $Y \pm l_{\text {cycl }}$, the guiding center at $Y$ acquires an $\mathbf{E} \times \mathbf{B}$ drift in the direction of the arrows (The drift along nonextremal parts of the orbit averages out, approximately ) A resonance occurs if the drift at one extremal point reinforces the drift at the other, as shown (b) Numerically calculated trajectories for a sinusoidal potential $(\varepsilon=0015)$ The horizontal lines are equipotentials at integer $y / a$ On resonance $\left(2 l_{\text {cycl }} / a=625\right)$ the guiding center drift is maximal, off resonance $\left(2 l_{\text {cycl }} / a=575\right)$ the drift is negligible Taken from C W J Beenakker, Phys Rev Lett 62, 2020 (1989)

The term $\delta D$ is an additional contribution to the $x x$-element of the unperturbed diffusion tensor $\mathbf{D}^{0}$, given by $D_{x x}^{0}=D_{y y}^{0}=D_{0}, D_{x y}^{0}=-D_{x y}^{0}=$ $-\omega_{\mathrm{c}} \tau D_{0}$, with $D_{0} \equiv \frac{1}{2} \tau v_{\mathrm{F}}^{2}\left[1+\left(\omega_{\mathrm{c}} \tau\right)^{2}\right]^{-1}$ (cf. Section 4.c). At this point we assume that for $l_{\text {cycl }} \ll l$ the contribution $\delta D$ from the guiding center drift is the dominant effect of the potential grating on the diffusion tensor D. A justification of this assumption requires a more systematic analysis of the transport problem, which is given in Ref. 111. Once $\mathbf{D}$ is known, the resistivity 
tensor $\boldsymbol{\rho}$ follows from the Einstein relation $\boldsymbol{\rho}=\mathbf{D}^{-1} / e^{2} \rho\left(E_{\mathrm{F}}\right)$, with $\rho\left(E_{\mathrm{F}}\right)$ the $2 \mathrm{D}$ density of states (cf Section $4 \mathrm{~b}$ ) Because of the large off-diagonal components of $\mathbf{D}^{0}$, an additional contribution $\delta D$ to $D_{x x}^{0}$ modifies predominantly $\rho_{y y} \equiv \rho_{\perp}$. To leading order in $\varepsilon$, one finds that

$$
\frac{\rho_{\perp}}{\rho_{0}}=1+2 \varepsilon^{2}\left(\frac{l^{2}}{a l_{\mathrm{cycl}}}\right) \cos ^{2}\left(2 \pi \frac{l_{\mathrm{cycl}}}{a}-\frac{\pi}{4}\right)
$$

with $\rho_{0}=m / n_{\mathrm{s}} e^{2} \tau$ the unperturbed resistivity A rigorous solution ${ }^{111}$ of the Boltzmann equation (for a $B$-independent scattering time) confirms this simple result in the regime $a \ll l_{\text {cycl }} \ll l$ and 1 s shown in Fig 37 to be in quite good agreement with the experimental data of Weiss et al ${ }^{255}$ Similar theoretical results have been obtained by Gerhardts et l $^{257}$ and by Winkler et al $^{258}$ (using an equivalent quantum mechanical formulation, see below)

As illustrated by the arrows in Fig 37, the maxima in $\rho_{\perp}$ are not at integer $2 l_{\text {cycl }} / a$, but shifted somewhat toward lower magnetic fields This phase shift is a consequence of the finite extension of the segment of the orbit around the extremal points $Y \pm l_{\text {cycl }}$, which contributes to the guiding center drift $v_{\text {drift }}(Y)$ Equation (11 4) implies that $\rho_{\perp}$ in a sinusoidal potential gratıng has minıma and maxima at approximately

$$
\begin{aligned}
2 l_{\text {cycl }} / a(\text { mınıma }) & =n-\frac{1}{4}, \\
2 l_{\text {cycl }} / a(\text { maxıma }) & =n+\frac{1}{4}-\operatorname{order}(1 / n),
\end{aligned}
$$

with $n$ an integer We emphasize that the phase shift is not universal, but depends on the functional form of $V(y)$ The fact that the experimental phase shift in Fig 37 agrees so well with the theory indicates that the actual potential gratıng in the experiment of Weiss et al is well modeled by a sinusoidal potential The maxima in $\rho_{\perp} / \rho_{0}$ have amplitude $\varepsilon^{2}\left(l^{2} / a l_{\text {cycl }}\right)$, which for a large mean free path $l$ can be of order unity, even if $\varepsilon \ll 1$ The guidingcenter-drift resonance thus explains the surprising experimental finding that a periodic modulation of the Fermi velocity of order $10^{-2}$ can double the resistivity

At low magnetic fields the experimental oscillations are damped more rapidly than the theory would predict, and, moreover, an unexplained positıve magnetoresistance is observed around zero field in $\rho_{\perp}$ (but not in $\rho_{\|}$) Part of this disagreement may be due to nonuniformities in the potential gratıng, which become especially important at low fields when the cyclotron orbit overlaps many modulation periods At high magnetıc fields $B \gtrsim 04 \mathrm{~T}$ the experımental data show the onset of Shubnikov-De Haas oscillations, which are a consequence of oscillations in the scattering time $\tau$ due to Landau 
level quantization (cf Section $4 \mathrm{c}$ ) This effect is neglected in the semiclassical analysis, which assumes a constant scattering time

The quantum mechanical $B$-dependence of $\tau$ also leads to weak-field oscillations in $\rho_{\|}$, with the same periodicity as the oscillations in $\rho_{\perp}$ discussed earler, but of much smaller amplitude and shifted in phase (see Fig 37, where a maximum in the experımental $\sigma_{\|}$around $03 \mathrm{~T}$ lines up with a minımum in $\rho_{\perp}$ ) These small antiphase oscillations in $\rho_{\| \mid}$were explained by Vasilopoulos and Peeters ${ }^{227}$ and by Gerhardts and Zhang ${ }^{259}$ as resulting from oscillations in $\tau$ due to the oscillatory Landau bandwidth The Landau levels $E_{n}=\left(n-\frac{1}{2}\right) \hbar \omega_{\mathrm{c}}$ broaden into a band of finite width in a periodic potential ${ }^{261}$ This Landau band is described by a dispersion law $E_{n}(k)$, where the wave number $k$ is related to the classical orbit center $(X, Y)$ by $k=Y e B / \hbar$ (cf the similar relation in Section 12) The classical guiding-center-drift resonance can also be explained in these quantum mechanical terms, if one so desıres, by noticing that the bandwidth of the Landau levels is proportional to the rootmean-square average of $v_{\mathrm{drift}}=d E_{n}(k) / \hbar d k$ A maximal bandwidth thus corresponds to a maximal guiding center drift and, hence, to a maximal $\rho_{\perp} \mathrm{A}$ maximum in the bandwidth also implies a minımum in the density of states at the Fermi level and, hence, a maximum in $\tau$ [Eq (4 28)] A maxumal bandwidth thus corresponds to a mınımal $\rho_{\|}$, whereas the $B$-dependence of $\tau$ can safely by neglected for the oscillations in $\rho_{\perp}$ (which are dominated by the classical guiding-center-drift resonance)

In a 2D periodic potential (a grid), the guidıng center drift domınates the magnetoresistivity in both diagonal components of the resistivity tensor Classically, the effect of a weak periodic potential $V(x, y)$ on $\rho_{x x}$ and $\rho_{y y}$ decouples if $V(x, y)$ is separable into $V(x, y)=f(x)+g(y)$ For the $2 \mathrm{D}$ sinusoidal potential $V(x, y) \propto \sin (2 \pi x / a)+\sin (2 \pi y / b)$, one finds that the effect of the grid is simply a superposition of the effects for two perpendicular gratıngs of periods $a$ and $b$ (No such decoupling occurs quantum mechan1cally ${ }^{254}$ ) Experiments by Alves et al ${ }^{262}$ and by Weiss et al ${ }^{263}$ confirm this expectation, except for a disagreement in the phase of the oscillations As noted, however, the phase is not universal but depends on the form of the periodic potential, which need not be sinusoidal

Because of the predominance of the classical guiding-center-drift resonance in a weak periodic potentıal, magnetotransport experiments are not well suited to study miniband structure in the density of states Magnetocapa-

${ }^{260}$ G Knorr, F R Hansen, J P Lynov, H L Pecselı, and J J Rasmussen, Physica Scripta 38, 829 (1988)

${ }^{261}$ A V Chaplik, Soltd State Comm, 53, 539 (1985)

${ }^{262}$ E S Alves, P H Beton, M Henını, L Eaves, P C Main, O H Hughes, G A Toombs, S P

Beaumont, and C D W Wilkınson, $J$ Phys Condens Matter 1, 8257 (1989)

${ }^{263}$ D Weiss K von Klitzing, G Ploog, and G Wermann, Surf $S c l$ (to be published) 
citance measurements ${ }^{256264265}$ are a more direct means of investigation, but somewhat outside the scope of this review

\section{Ballistic Transport}

\section{Conduction as a Transmission Problem}

In the ballistic transport regime, it is the scattering of electrons at the sample boundaries which limits the current, rather than impurity scattering The canonical example of a ballistic conductor is the point contact illustrated in Fig 7c The current $I$ through the narrow constriction in response to a voltage difference $V$ between the wide regions to the left and right is finite even in the absence of impurities, because electrons are scattered back at the entrance of the constriction The contact conductance $G=I / V$ is proportional to the constriction width but independent of its length One cannot therefore describe the contact conductance in terms of a local conductivity, as one can do in the diffusive transport regime Consequently, the Einstein relation (4 10) between the conductivity and the diffusion constant at the Fermi level, of which we made use repeatedly in Section II, is not applicable in that form to determine the contact conductance The Landauer formula is an alternative relation between the conductance and a Fermi level property of the sample, without the restriction to diffusive transport We discuss this formulation of conduction in Section 122 The Landauer formula expresses the conductance in terms of transmission probabilities of propagating modes at the Fermı level (also referred to as quantum channels in this context) Some elementary properties of the modes are summarized in Section $12 \mathrm{a}$

\section{a Electron Waveguide}

We consider a conductıng channel in a 2DEG (an "electron waveguide"), defined by a lateral confining potential $V(x)$, in the presence of a perpendicular magnetic field $B$ (in the $z$-direction) In the Landau gauge $\mathbf{A}=(0, B x, 0)$ the hamiltonian has the form

$$
\mathscr{H}=\frac{p_{x}^{2}}{2 m}+\frac{\left(p_{y}+e B x\right)^{2}}{2 m}+V(x)
$$

for a single spin component (cf Section 10a) Because the canonical momentum $p_{y}$ along the channel commutes with $\mathscr{H}$, one can diagonalize $p_{y}$ and $\mathscr{H}$ simultaneously For each eigenvalue $\hbar k$ of $p_{y}$, the hamiltonian (12 1)

\footnotetext{
${ }^{264} \mathrm{~K}$ Isma1l T P Smith III, W T Masselnk, and H I Smith, Appl Phys Lett 55, 2766(1989) ${ }^{265}$ W Hansen, T P Smith, III, $K$ Y Lee, J A Brum, C M Knoedler, J M Hong, and D P Kern, Phys Rev Lett 62, 2168 (1989)
} 
has a discrete spectrum of energy eigenvalues $E_{n}(k), n=1,2, \quad$, corresponding to eigenfunctions of the form

$$
|n, k\rangle=\Psi_{n k}(x) \mathrm{e}^{i k y}
$$

In waveguide terminology, the index $n$ labels the modes, and the dependence of the energy (or "frequency") $E_{n}(k)$ on the wave number $k$ is the dispersion relation of the $n$th mode A propagating mode at the Fermi level has cutoff frequency $E_{n}(0)$ below $E_{\mathrm{F}}$ The wave function (122) is the product of a transverse amplitude profile $\Psi_{n k}(x)$ and a longitudinal plane wave $\mathrm{e}^{i k y}$ The average velocity $v_{n}(k)$ along the channel in state $|n, k\rangle$ is the expectation value of the $y$-component of the velocity operator $\mathbf{p}+e \mathbf{A}$

$$
v_{n}(k) \equiv\left\langle n, k\left|\frac{p_{y}+e A_{y}}{m}\right| n, k\right\rangle=\left\langle n, k\left|\frac{\partial \mathscr{H}}{\partial p_{y}}\right| n, k\right\rangle=\frac{d E_{n}(k)}{\hbar d k}
$$

For a zero magnetic field, the dispersion relation $E_{n}(k)$ has the simple form (43) The group velocity $v_{n}(k)$ is then simply equal to the velocity $\hbar k / m$ obtained from the canonical momentum This equality no longer holds in the presence of a magnetic field, because the canonical momentum contains an extra contribution from the vector potential The dispersion relation in a nonzero magnetic field was derived in Section $10 \mathrm{a}$ for a parabolic confining potential $V(x)=\frac{1}{2} m \omega_{0}^{2} x^{2}$ From Eq (105) one calculates a group velocity $\hbar k / M$ that is smaller than $\hbar k / m$ by a factor of $1+\left(\omega_{\mathrm{c}} / \omega_{0}\right)^{2}$

Insight into the nature of the wave functions in a magnetic field can be obtained from the correspondence with classical trajectories These are most easily visualized in a square-well confining potential, as we now discuss (following Ref 266) The position $(x, y)$ of an electron on the circle with center coordinates $(X, Y)$ can be expressed in terms of its velocity $\mathbf{v}$ by

$$
x=X+v_{y} / \omega_{c}, \quad y=Y-v_{x} / \omega_{c},
$$

with $\omega_{\mathrm{c}} \equiv e B / m$ the cyclotron frequency The cyclotron radius is $(2 m E)^{1 / 2} / e B$, with $E \equiv \frac{1}{2} m v^{2}$ the energy of the electron Both the energy $E$ and the separation $X$ of the orbit center from the center of the channel are constants of the motion The coordinate $Y$ of the orbit center parallel to the channel walls changes on each specular reflection One can classify a trajectory as a cyclotron orbit, skipping orbit, or traversing trajectory, depending on whether the trajectory collides with zero, one, or both channel walls $\operatorname{In}(X, E)$ space these three types of trajectories are separated by the two parabolas $(X \pm W / 2)^{2}=2 m E(e B)^{2}$ (Fig 39) The quantum mechanical dispersion relation $E_{n}(k)$ can be drawn into this classical "phase diagram" because of the correspondence $k=-X e B / \hbar$ This correspondence exists because both $k$ and 


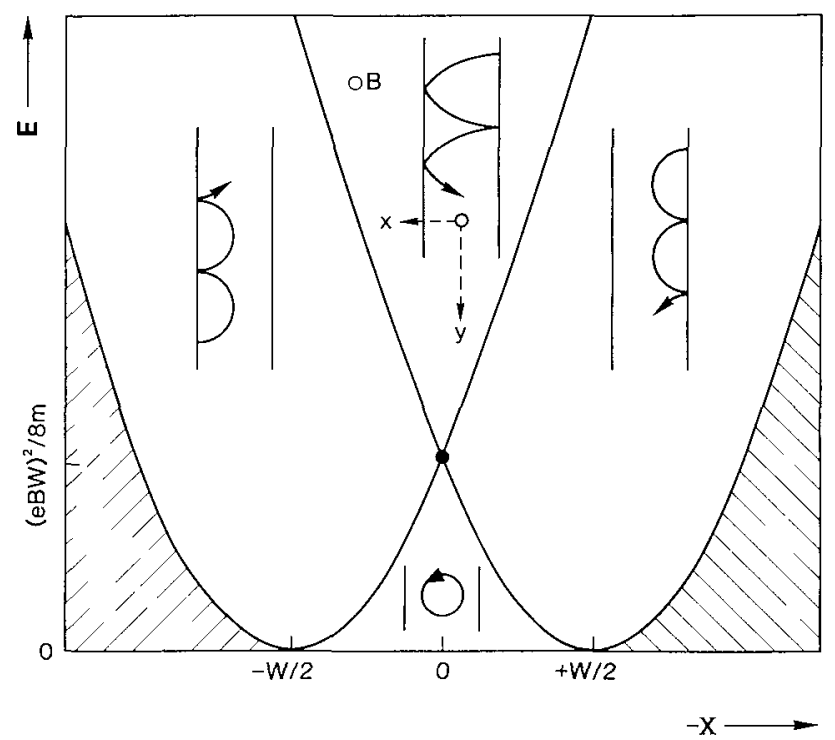

Fig 39 Energy-orbit center phase space The two parabolas divide the space into four regions, which correspond to different types of classical trajectories in a magnetic field (clockwise from left skipping orbits on one edge, traversing trajectories, skipping orbits on the other edge, and cyclotron orbits) The shaded region is forbidden The region at the upper center contains traversing trajectories moving in both directions, but only one region is shown for clarity Taken from C W J Beenakker et al, Superlattices and Microstructures 5, 127 (1989)

$X$ are constants of the motion and it follows from the fact that the component $\hbar k$ along the channel of the canonical momentum $\mathbf{p}=m \mathbf{v}-e \mathbf{A}$ equals

$$
\hbar k=m v_{y}-e A_{y}=m v_{y}-e B x=-e B X
$$

in the Landau gauge.

In Fig. 40 we show $E_{n}(k)$ both in weak and in strong magnetic fields, calculated $^{266}$ for typical parameter values from the Bohr-Sommerfeld quantization rule discussed here. The regions in phase space occupied by classical skipping orbits are shaded. The unshaded regions contain cyclotron orbits (at small $E$ ) and traversing trajectories (at larger $E$ ) (cf. Fig. 39). The cyclotron orbits correspond quantum mechanically to states in Landau levels. These are the flat portions of the dispersion relation at energies $E_{n}=\left(n-\frac{1}{2}\right) \hbar \omega_{\mathrm{c}}$. The group velocity (12.3) is zero in a Landau level, as one would expect from the correspondence with a circular orbit. The traversing trajectories correspond to states in magnetoelectric subbands, which interact

${ }^{266} \mathrm{C}$ W J Beenakker, H van Houten, and B J van Wees, Superlattices and Microstructures $\mathbf{5}$, 127 (1989) 

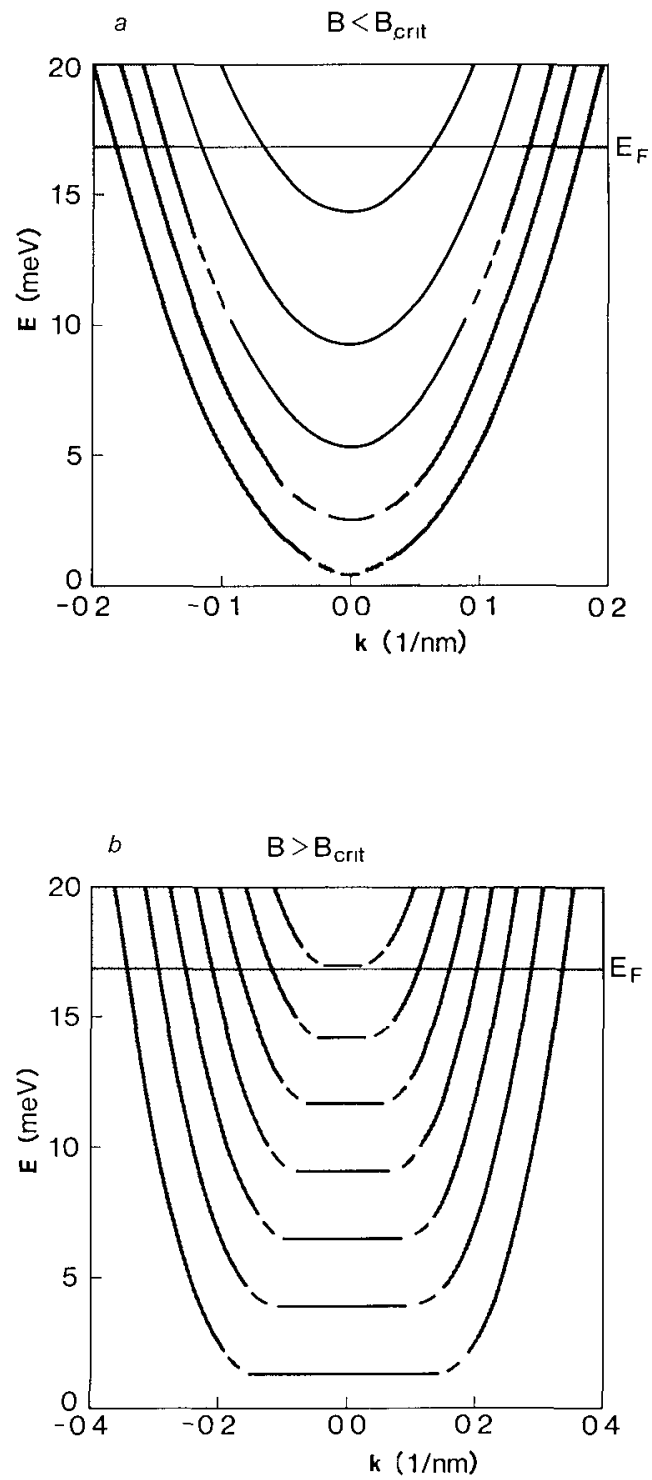

FiG 40 Disperston relation $E_{n}(k)$, calculated for parameters (a) $W=100 \mathrm{~nm}, B=1 \mathrm{~T}$, (b) $W=200 \mathrm{~nm}, B=15 \mathrm{~T}$ The horizontal line at $17 \mathrm{meV}$ indicates the Fermi energy The shaded area is the region of classical skipping orbits and is bounded by the two parabolas shown in Fig 39 (with the correspondence $k=-X e B / h$ ) Note that in (a) edge states coexist at the Fermi level with states interacting with both boundaries $\left(B<B_{\text {crit }} \equiv 2 h k_{\mathrm{F}} / e B\right)$, while in (b) all states at the Fermi level interact with one boundary only $\left(B>B_{\text {crnt }}\right)$ Taken from C W J Beenakker et al, Superlattices and Microstructures 5, 127 (1989) 
with both the opposite channel boundaries and have a nonzero group velocity The skipping orbits correspond to edge states, which interact with a single boundary only The two sets of edge states (one for each boundary) are disjunct in $(k, E)$ space Edge states at opposite boundaries move in opposite directions, as is evident from the correspondence with skipping orbits or by inspection of the slope of $E_{n}(k)$ in the two shaded regions in Fig 40

If the Fermı level lies between two Landau levels, the states at the Fermı level consist only of edge states if $B>B_{\text {crit }}$, as in Fig $40 \mathrm{~b}$ The "critical" field $B_{\text {crit }}=2 \hbar k_{\mathrm{F}} / e W$ is obtained from the classical correspondence by requiring that the channel width $W$ should be larger than the cyclotron diameter $2 \hbar k_{\mathrm{F}} / e B$ at the Ferm level This is the same characteristic field that played a role in the discussion of magneto size effects in Sections 5 and 10 At fields $B<B_{\text {crit }}$, as in Fig 40a, edge states coexist at the Fermı level with magnetoelectric subbands In still lower fields $B<B_{\text {thres }}$ all states at the Fermi level interact with both edges The criterion for this is that $W$ should be smaller than the transverse wavelength ${ }^{267} \lambda_{\mathrm{t}}=\left(\hbar / 2 k_{\mathrm{F}} e B\right)^{1 / 3}$ of the edge states, so the threshold field $B_{\text {thres }} \sim \hbar / e k_{\mathrm{F}} W^{3}$ Contrary to initial expectations, ${ }^{268}$ this lower characteristic field does not appear to play a decisive role in magneto size effects

A quick way to arrive at the dispersion relation $E_{n}(k)$, which is sufficiently accurate for our purposes, is to apply the Bohr-Sommerfeld quantization rule $^{80269}$ to the classical motion in the $x$-direction

$$
\frac{1}{\hbar} \oint p_{x} d x+\gamma=2 \pi n, \quad n=1,2,
$$

The integral is over one period of the motion The phase shift $\gamma$ is the sum of the phase shifts acquired at the two turning points of the projection of the motion on the $x$-axis The phase shift upon reflection at the boundary is $\pi$, to ensure that incident and reflected waves cancel (we consider an infinite barrier potential at which the wave function vanishes) The other turning points (at which $v_{x}$ varies smoothly) have a phase shift of $-\pi / 2^{93}$ Consequently, for a traversing trajectory $\gamma=\pi+\pi=0(\bmod 2 \pi)$, for a skipping orbit $\gamma=\pi-\pi / 2=\pi / 2$, and for a cyclotron orbit $\gamma=-\pi / 2-\pi / 2=\pi$ $(\bmod 2 \pi)$ In the Landau gauge one has $p_{x}=m v_{x}=e B(Y-y)$, so Eq (126) takes the form

$$
B \oint(Y-y) d x=\frac{h}{e}\left(n-\frac{\gamma}{2 \pi}\right)
$$

${ }^{267} \mathrm{R}$ E Prange and T-W Nee, Phys Rev 168779 (1968)

${ }^{268} \mathrm{C}$ W J Beenakker and $\mathrm{H}$ van Houten, Phys Rev Lett 60, 2406 (1988)

${ }^{269} \mathrm{~A}$ M Kosevich and I M Lifshitz, Zh Eksp Teor Flz 29,743 (1955) [Sov Phys JETP 2, 646 (1956)], M S Kha1kin, Adv Phys 18, 1 (1969) 
a

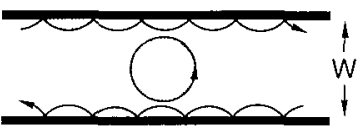

b

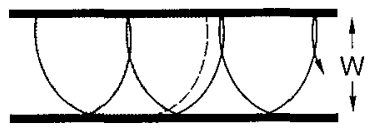

Fig. 41 Classical trajectories in a magnetic field The flux through the shaded area is quantized according to the Bohr-Sommerfeld quantization rule (12.7) The shaded area in (b) is bounded by the channel walls and the circle formed by the continuation (dashed) of one circular arc of the traversing trajectory

This quantization condition has the appealing geometrical interpretation that $n-\gamma / 2 \pi$ flux quanta $h / e$ are contained in the area bounded by the channel walls and a circle of cyclotron radius $(2 m E)^{1 / 2} / e B$ centered at $X$ (cf. Fig. 41). It is now straightforward to find for each integer $n$ and coordinate $X$ the energy $E$ that satisfies this condition. The dispersion relation $E_{n}(k)$ then follows on identifying $k=-X e B / \hbar$, as shown in Fig. 40.

The total number $N$ of propagating modes at energy $E$ is determined by the maximum flux $\Phi_{\max }$ contained in an area bounded by the channel walls and a circle of radius $(2 m E)^{1 / 2} / e B$, according to $N=\operatorname{Int}\left[e \Phi_{\max } / h+\gamma / 2 \pi\right]$. Note that a maximal enclosed flux is obtained by centering the circle on the channel axis. Some simple geometry then leads to the result ${ }^{80}(10.8)$, which is plotted together with that for a parabolic confinement in Fig. 31. Equation (10.8) has a discontinuity at magnetic fields for which the cyclotron diameter equals the channel width, due to the jump in the phase shift $\gamma$ as one goes from a cyclotron orbit to a traversing trajectory. This jump is an artifact of the present semiclassical approximation in which the extension of the wave function beyond the classical orbit is ignored. Since the discontinuity in $N$ is at most \pm 1 , it is unimportant in many applications. More accurate formulas for the phase shift $\gamma$, which smooth out the discontinuity, have been derived in Ref. 270. If necessary, one can also use more complicated but exact solutions of the Schrödinger equation, which are known. ${ }^{267}$

\section{b. Landauer Formula}

Imagine two wide electron gas reservoirs having a slight difference $\delta n$ in electron density, which are brought into contact by means of a narrow channel, as in Fig. 42a. A diffusion current $J$ will flow in the channel, carried by electrons with energies between the Fermi energies $E_{\mathrm{F}}$ and $E_{F}+\delta \mu$ in the low- and high-density regions. For small $\delta n$, one can write for the Fermi energy difference (or chemical potential difference) $\delta \mu=\delta n / \rho\left(E_{\mathrm{F}}\right)$, with $\rho\left(E_{\mathbf{F}}\right)$ the density of states at $E_{\mathrm{F}}$ in the reservoir (cf. Section 4.1). The diffusion 
constant (or "diffusance") ${ }^{4} \tilde{D}$ is defined by $J=\widetilde{D} \delta n$ and is related to the conductance $G$ by

$$
G=e^{2} \rho\left(E_{\mathrm{F}}\right) \tilde{D}
$$

Equation (128) generalizes the Einstein relation (4 10) and is derived in a completely analogous way [by requiring that the sum of drift current $G V / e$ and diffusion current $\tilde{D} \delta n$ be zero when the sum of the electrostatic potential difference $e V$ and chemical potential difference $\delta n / \rho\left(E_{\mathrm{F}}\right)$ vanishes]

Since the diffusion current (at low temperatures) is carried by electrons within a narrow range $\delta \mu$ above $E_{\mathrm{F}}$, the diffusance can be expressed in terms of Fermi level properties of the channel (see below) The Einstem relation (128) then yields the required Fermı level expression of the conductance This by no means implies that the drift current induced by an electrostatic potential difference is carried entirely by electrons at the Fermi energy To the contrary, all electrons (regardless of therr energy) acquire a nonzero drift velocity in an electric field This point has been the cause of some confusion in the literature on the quantum Hall effect, so we will return to it in Section $18 \mathrm{c}$ In the following we will refer to electrons at the Fermi energy as "current-carrying electrons" and show that "the current in the channel is shared equally among the modes at the Fermi level" These and similar statements should be interpreted as referring to the diffusion problem, where the current is induced by density differences without an electric field We make no attempt here to evaluate the distribution of current in response to an electric field in a system of uniform density That is a difficult problem, for which one has to determine the electric field distribution self-consistently from Poisson's and Boltzmann's equations Such a calculation for a quantum point contact has been performed in Refs 271 and 272 Fortunately, there 15 no need to know the actual current distribution to determine the conductance, in view of the Einstein relation (128) The distribution of current (and electric field) is of importance only beyond the regime of a linear relation between current and voltage We will not venture beyond this linear response regime

To calculate the diffusance, we first consider the case of an ideal electron waveguide between the two reservoirs By "ideal" it is meant that within the waveguide the states with group velocity pointing to the right are occupied up to $E_{\mathrm{F}}+\delta \mu$, while states with group velocity to the left are occupied up to $E_{\mathrm{F}}$ and empty above that energy (cf $\mathrm{F}_{1} \mathrm{~g} 42 \mathrm{~b}$ ) This requires that an electron near the Fermi energy that is injected into the waveguide by the reservoir at $E_{\mathrm{F}}+\delta \mu$ propagates into the other reservoir without being reflected (The

${ }^{271}$ I B Levinson, $Z$ h Eksp Teor Flz 95, 2175 (1989) [Sov Phys JETP 68, 1257 (1989)]

${ }^{272}$ M C Payne, J Phys Condens Matter 1, 4931 (1989), 4939 (1989) 


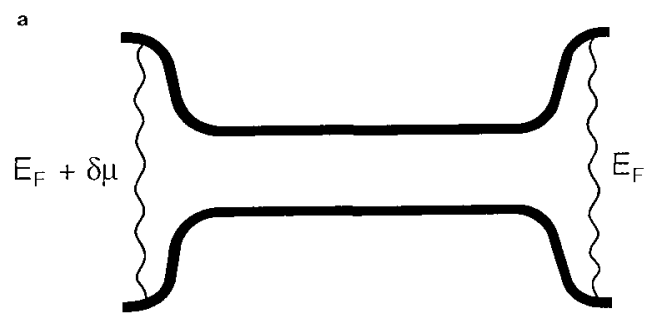

b

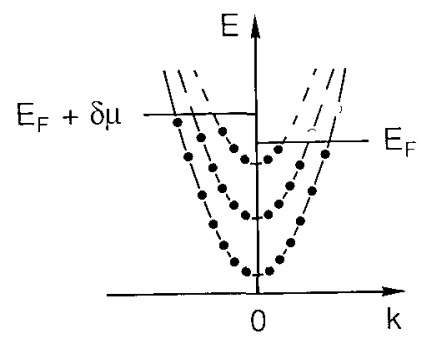

FIG. 42 (a) Narrow channel connecting two wide electron gas regions, having a chemical potential difference $\delta \mu$. (b) Schematic dispersion relation in the narrow channel. Left-moving states $(k>0)$ are filled up to chemical potential $E_{\mathrm{F}}$, right-moving states up to $E_{\mathrm{F}}+\delta \mu$ (solid dots). Higher-lying states are unoccupied (open dots).

physical requirements for this to happen will be discussed in Section 13.) The amount of diffusion current per energy interval carried by the right-moving states (with $k<0$ ) in a mode $n$ is the product of density of states $\rho_{n}^{-}$and group velocity $v_{n}$. Using Eqs. (4.4) and (12.3), we find the total current $J_{n}$ carried by that mode to be

$$
J_{n}=\int_{E_{\mathrm{F}}}^{E_{\mathrm{F}}+\delta \mu} g_{\mathrm{s}} g_{\mathrm{v}}\left(2 \pi \frac{d E_{n}(k)}{d k}\right)^{-1} \frac{d E_{n}(k)}{\hbar d k}=\frac{g_{\mathrm{s}} g_{\mathrm{v}}}{h} \delta \mu,
$$

independent of mode index and Fermi energy. The current in the channel is shared equally among the $N$ modes at the Fermi level because of the cancellation of group velocity and density states. We will return to this equipartition rule in Section 13, because it is at the origin of the quantization $^{6,7}$ of the conductance of a point contact.

Scattering within the narrow channel may reflect part of the injected current back into the left reservoir. If a fraction $T_{n}$ of $J_{n}$ is transmitted to the reservoir at the right, then the total diffusion current in the channel becomes $J=(2 / h) \delta \mu \sum_{n=1}^{N} T_{n}$. (Unless stated otherwise, the formulas in the remainder of this review refer to the case $g_{\mathrm{s}}=2, g_{\mathrm{v}}=1$ of twofold spin degeneracy and a 
single valley, appropriate for most of the experıments ) Using $\delta \mu=\delta n / \rho\left(E_{F}\right)$, $J=\tilde{D} \delta n$, and the Einstein relation (128), one obtains the result

$$
G=\frac{2 e^{2}}{h} \sum_{n-1}^{N} T_{n}
$$

which can also be written in the form

$$
G=\frac{2 e^{2}}{h} \sum_{n=1}^{N}\left|t_{m n}\right|^{2} \equiv \frac{2 e^{2}}{h} \operatorname{Tr} \mathbf{t} \mathbf{\dagger}
$$

where $T_{n}=\sum_{m=1}^{N}\left|t_{m n}\right|^{2}$ is expressed in terms of the matrix $\mathbf{t}$ of transmission probability amplitudes from mode $n$ to mode $m$ This relation between conductance and transmission probabilities at the Fermi energy is referred to as the Landauer formula because of Landauer's pioneering 1957 paper ${ }^{4}$ Derivations of Eq (1210) based on the Kubo formula of linear response theory have been given by several authors, both for zero ${ }^{143273274}$ and nonzero 275276 magnetic fields The identification of $G$ as a contact conductance is due to Imry $^{1}$ In earlier work Eq (1210) was considered suspect ${ }^{277} 279$ because it gives a finite conductance for an ideal (ballistic) conductor, and alternative expressions were proposed ${ }^{188} 280282$ that were considered to be more realistic (In one dimension these alternative formulas reduce to the original Landauer formula ${ }^{4} G=\left(e^{2} / h\right) T(1-T)^{-1}$, which gives infinite conductance for unit transmission since the contact conductance $e^{2} / h$ is ignored ${ }^{1}$ ) For historical accounts of this controversy, from two different points of view, we refer the interested reader to papers by Landauer ${ }^{283}$ and by Stone and Szafer ${ }^{274}$ We have briefly mentioned this now-settled controversy, because it sheds some light onto why the quantization of the contact conductance had not been predicted theoretically prior to its experimental discovery in 1987

Equation (12 10) refers to a two-terminal resistance measurement, in which the same two contacts (modeled by reservors in Fig 42a) are used to drive a current through the system and to measure the voltage drop More generally, one can consider a multireservoir conductor as in Fig 43 to model, for

\footnotetext{
${ }^{273}$ E N Economou and C M Soukoulıs, Phys Rev Lett 46, 618 (1981)

${ }^{274}$ A D Stone and A Szafer, IBM J Res Dev 32, 384 (1988)

${ }^{275} \mathrm{~J}$ Kucera and P Streda $J$ Phys $C 21,4357$ (1988)

${ }^{276} \mathrm{H}$ U Baranger and A D Stone, Phys Rev B 40, 8169 (1989)

${ }^{277}$ D J Thouless, Phys Rev Lett 47, 972 (1981)

${ }^{278} \mathrm{R}$ Landauer, Phys Lett A 85, 91 (1981)

${ }^{279}$ E N Engquist and P W Anderson, Phys Rev B 24, 1151 (1981)

${ }^{280} \mathrm{P}$ W Anderson, D J Thouless, E Abrahams, and D S Fisher, Phys Rev B 22, 3519 (1980)

${ }^{281}$ D C Langreth and E Abrahams, Phys Rev B 24, 2978 (1981)

${ }^{282}$ M Ya Azbel, $J$ Phys C 14, L225 (1981)

${ }^{283} \mathrm{R}$ Landauer, $J$ Phys Condens Matter 1, 8099 (1989), also in Ref 15
} 


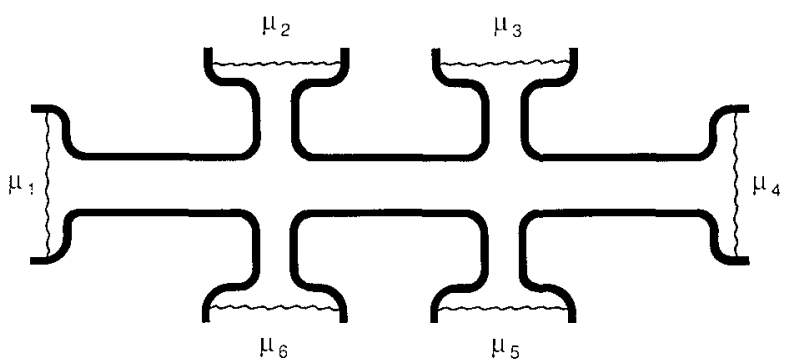

FIG. 43. Generalization of the geometry of Fig, 42a to a multireservoir conductor.

example, four-terminal resistance measurements in which the current source and drain are distinct from the voltage probes. The generalization of the Landauer formula (12.10) to multiterminal resistances is due to Büttiker. ${ }^{5}$ Let $T_{\alpha \rightarrow \beta}$ be the total transmission probability from reservoir $\alpha$ to $\beta$ :

$$
T_{\alpha \rightarrow \beta}=\sum_{n=1}^{N_{\alpha}} \sum_{m=1}^{N_{\beta}}\left|t_{\beta \alpha, m n}\right|^{2} .
$$

Here $N_{\alpha}$ is the number of propagating modes in the channel (or "lead") connected to reservoir $\alpha$ (which in general may be different from the number $N_{\beta}$ in lead $\beta$ ), and $t_{\beta \alpha, m n}$ is the transmission probability amplitude from mode $n$ in lead $\alpha$ to mode $m$ in lead $\beta$. The leads are modeled by ideal electron waveguides, in the sense discussed before, so that the reservoir $\alpha$ at chemical potential $\mu_{\alpha}$ above $E_{\mathrm{F}}$ injects into lead $\alpha$ a (charge) current $(2 e / h) N_{\alpha} \mu_{\alpha}$. A fraction $T_{\alpha \rightarrow \beta} / N_{\alpha}$ of that current is transmitted to reservoir $\beta$, and a fraction $T_{\alpha \rightarrow \alpha} / N_{\alpha} \equiv R_{\alpha} / N_{\alpha}$ is reflected back into reservoir $\alpha$, before reaching one of the other reservoirs. The net current $I_{\alpha}$ in lead $\alpha$ is thus given by ${ }^{5}$

$$
\frac{h}{2 e} I_{\alpha}=\left(N_{\alpha}-R_{\alpha}\right) \mu_{\alpha}-\sum_{\beta(\beta \neq \alpha)} T_{\beta \rightarrow \alpha} \mu_{\beta}
$$

The chemical potentials of the reservoirs are related to the currents in the leads via a matrix of transmission and reflection coefficients. The sums of columns or rows of this matrix vanish:

$$
\begin{aligned}
& N_{\alpha}-R_{\alpha}-\sum_{\beta(\beta \neq \alpha)} T_{\alpha \rightarrow \beta}=0, \\
& N_{\alpha}-R_{\alpha}-\sum_{\beta(\beta \neq \alpha)} T_{\beta \rightarrow \alpha}=0 .
\end{aligned}
$$

Equation (12.13) follows from current conservation, and Eq. (12.14) follows from the requirement that an increase of all the chemical potentials by the same amount should have no effect on the net currents in the leads.

Equation (12.12) can be applied to a measurement of the four-terminal 
resistance $R_{\alpha \beta \gamma \delta}=V / I$, in which a current $I$ flows from contact $\alpha$ to $\beta$ and a voltage difference $V$ is measured between contacts $\gamma$ and $\delta$ Setting $I_{\alpha}=I=-I_{\beta}$, and $I_{\alpha}=0$ for $\alpha^{\prime} \neq \alpha, \beta$, one can solve the set of linear equations (1212) to determine the chemical potential difference $\mu_{\gamma}-\mu_{\delta}$ (Only the differences in chemical potentials can be obtained from the $n$ equations (12 12), which are not independent in view of Eq (1214) By fixing one chemical potential at zero, one reduces the number of equations to $n-1$ independent ones ) The four-terminal resistance $R_{\alpha \beta \gamma \delta}=\left(\mu_{\gamma}-\mu_{\delta}\right) / e I$ is then obtained as a rational function of the transmission and reflection probabilities We will refer to this procedure as the Landauer-Buttiker formalism It provides a unified description of the whole variety of electrical transport experiments discussed in this review

The transmission probabilities have the symmetry

$$
t_{\beta \alpha n m}(B)=t_{\alpha \beta m n}(-B) \Rightarrow T_{\alpha \rightarrow \beta}(B)=T_{\beta \rightarrow \alpha}(-B)
$$

Equation (12 15) follows by combining the unitarity of the scattering matrix $\mathbf{t} \dagger=\mathbf{t}^{1}$, required by current conservation, with the symmetry $\mathbf{t}^{*}(-B)=\mathbf{t}^{-1}(B)$, required by time-reversal invariance $\left(^{*}\right.$ and $\dagger$ denote complex and Hermitian conjugation, respectively) As shown by Buttıker, ${ }^{5284}$ the symmetry (1215) of the coefficients in Eq (1212) implies a reciprocity relation for the four-terminal resistance

$$
R_{\alpha \beta \gamma \delta}(B)=R_{\gamma \delta \alpha \beta}(-B)
$$

The resistance is unchanged if current and voltage leads are interchanged with simultaneous reversal of the magnetic field direction A special case of Eq (12 16) is that the two-terminal resistance $R_{\alpha \beta \alpha \beta}$ is even in $B$ In the diffusive transport regime, the reciprocity relation for the resistance follows from the Onsager-Casimir relation ${ }^{285} \boldsymbol{\rho}(B)=\boldsymbol{\rho}^{\mathrm{T}}(-\beta)$ for the resistivity tensor ( $\mathrm{T}$ denotes the transpose) Equation (1216) holds also in cases that the concept of a local resistivity breaks down One experimental demonstration ${ }^{80}$ of the validity of the reciprocity relation in the quantum ballistic transport regime will be discussed in Section 14 Other demonstrations have been given in Refs 286-289 We emphasize that strict reciprocity holds only

${ }^{284} \mathrm{M}$ Buttıker, IBM J Res Dev 32, 317 (1988)

${ }^{285}$ H B G Casımır, Rev Mod Phys 17, 343 (1945), Philps Res Rep 1, 185 (1946), L Onsager, Phys Rev 38, 2265 (1931, see also S R de Groot and P Mazur, "Non-Equilibrium Thermodynamıs" Dover, New York, 1984

${ }^{286}$ A D Benott, S Washburn, C P Umbach, R B Lalbowitz, and R A Webb, Phys Rev Lett 57, 1765 (1986)

${ }^{287}$ H H Sample, W J Bruno, S B Sample, and E K Sichel, J Appl Phys 61, 1079 (1987)

${ }^{288} \mathrm{~L}$ L Soethout, $H$ van Kempen, J T P W van Maarseveen, P A Schroeder, and P Wyder, $J$ Phys F 17, L129 (1987)

${ }^{289} \mathrm{G}$ Timp, H U Baranger, $\mathrm{P}$ deVegvar, J E Cunningham, R E Howard, R Behrınger, and P M Mankıewich, Phys Rev Lett 60, 2081 (1988) 
in the linear response limit of infinitesimally small currents and voltages Deviations from Eq (12 16) can occur experimentally ${ }^{290}$ due to nonlınearities from quantum interference, ${ }^{146291}$ which in the case of a long phase coherence time $\tau_{\phi}$ persist down to very small voltages $V \gtrsim \hbar / e \tau_{\phi}$ Magnetic impurities can be another source of deviations from reciprocity if the applied magnetic field is not sufficiently strong to reverse the magnetic moments on field reversal The large $\pm B$ asymmetry of the two-terminal resistance of a point contact reported in Ref 292 has remained unexplained (see Section 21)

The scattering matrix $\mathrm{t}$ in Eq (1215) describes elastic scattering only Inelastic scattering is assumed to take place exclusively in the reservorrs That is a reasonable approximation at temperatures that are sufficiently low that the size of the conductor is smaller than the inelastic scattering length (or the phase coherence length if quantum interference effects play a role) Reservours thus play a dual role in the Landauer-Buttıker formalism On the one hand, a reservoir is a model for a current or voltage contact, on the other hand, a reservoir brings inelastic scattering into the system The reciprocity relation (12 16) is unaffected by adding reservoirs to the system and is not restricted to elastic scattering ${ }^{284}$ More realistic methods to include inelastic scattering in a distributed way throughout the system have been proposed, but are not yet implemented in an actual calculation 293294

\section{Quantum Point Contacts}

Many of the principal phenomena in ballistic transport are exhibited in the cleanest and most extreme way by quantum point contacts These are short and narrow constrictions in a 2DEG, with a width of the order of the Fermı wavelength 6759 The conductance of quantum point contacts is quantized in units of $2 e^{2} / h$ This quantization is reminiscent of the quantization of the Hall conductance, but is measured in the absence of a magnetic field The zero-field conductance quantization and the smooth transition to the quantum Hall effect on applying a magnetic field are essentially consequences of the equipartition of current among an integer number of propagating modes in the constriction, each mode carrying a current of $2 e^{2} / h$ times the applied voltage $V$ Deviations from precise quantization result from nonunit transmission probability of propagating modes and from nonzero transmission probability of evanescent (nonpropagating) modes Experıment

${ }^{290} \mathrm{P}$ G N de Vegvar, G Timp, P M Mankıewich, J E Cunnıngham, R Behrınger, and R E Howard, Phys Rev B 384326 (1988)

${ }^{291}$ A I Larkın and D E Khmel'nitskı, Zh Eksp Teor Ftz 91, 1815 (1986) [Sov Phys JETP 64, $1075(1986)]$

${ }^{292} \mathrm{P}$ H M van Loosdrecht, C W J Beenakker, $H$ van Houten, J G Williamson, B J van Wees, J E Moo1,, C T Foxon, and J J Harrıs, Phys Rev B 38, 10162 (1988)

${ }^{293}$ S Datta, Phys Rev B 40, 5830 (1989)

${ }^{294}$ S Feng Phys Lett A 143, 400 (1990) 
and theory in a zero magnetic field are reviewed in Section 13.a. The effect of a magnetic field is the subject of Section 13.b, which deals with depopulation of subbands and suppression of backscattering by a magnetic field, two phenomena that form the basis for an understanding of magnetotransport in semiconductor nanostructures.

\section{a. Conductance Quantization}

(1) Experiments. The study of electron transport through point contacts in metals has a long history, which goes back to Maxwell's investigations ${ }^{295}$ of the resistance of an orifice in the diffusive transport regime. Ballistic transport was first studied by Sharvin, ${ }^{296}$ who proposed and subsequently realized ${ }^{297}$ the injection and detection of a beam of electrons in a metal by means of point contacts much smaller than the mean free path. With the possible exception of the scanning tunneling microscope, which can be seen as a point contact on an atomic scale, ${ }^{298-303}$ these studies in metals are essentially restricted to the classical ballistic transport regime because of the extremely small Fermi wavelength $\left(\lambda_{\mathrm{F}} \approx 0.5 \mathrm{~nm}\right)$. Point contacts in a $2 \mathrm{DEG}$ cannot be fabricated by simply pressing two wedge- or needle-shaped pieces of material together (as in bulk semiconductors ${ }^{304}$ or metals ${ }^{305}$ ), since the electron gas is confined at the GaAs-AlGaAs interface in the interior of the heterostructure. Instead, they are defined electrostatically ${ }^{24,58}$ by means of a split gate on top of the heterostructure (a schematical cross-sectional view was given in Fig. 4b, while the micrograph Fig. 5b shows a top view of the split gate of a double-point contact device; see also the inset in Fig. 44). In this way one can define short and narrow constrictions in the $2 \mathrm{DEG}$, of variable width $0 \lesssim W \lesssim 250 \mathrm{~nm}$ comparable to the Fermi wavelength $\lambda_{\mathrm{F}} \approx 40 \mathrm{~nm}$ and much shorter than the mean free path $l \approx 10 \mu \mathrm{m}$.

Van Wees et al. ${ }^{6}$ and Wharam et al. ${ }^{7}$ independently discovered a sequence of steps in the conductance of such a point contact as its width was varied by means of the voltage on the split gate (see Fig. 44). The steps are near integer multiples of $2 e^{2} / h \approx(13 k \Omega)^{-1}$, after correction for a gate-voltageindependent series resistance from the wide $2 \mathrm{DEG}$ regions. An elementary

\footnotetext{
${ }^{295} \mathrm{~J}$ C Maxwell, "A Treatise on Electricity and Magnetısm " Clarendon, Oxford, 1904

${ }^{296} \mathrm{Yu}$ V Sharvin, $Z h$ Eksp Teor Flz 48, 984 (1965) [Sov Phys JETP 21, 655 (1965)]

${ }^{297} \mathrm{Yu}$ V Sharvin and N I Bogatına, Zh Eksp Teor Phys 56, 772 (1969) [Sov Phys JETP 29, 419 (1969)]

${ }^{298}$ J K Gimzewski and R Moller, Phys Rev B 36, 1284 (1987)

${ }^{299}$ N D Lang, Phys Rev B 36, 8173 (1987)

${ }^{300} \mathrm{~J}$ Ferrer, A Martın-Rodero, and F Flores, Phys Rev B 38, 10113 (1988)

${ }^{301}$ N D Lang, Comm Cond Matt Phys 14, 253 (1989)

${ }^{302}$ N D Lang, A Yacoby, and Y Imry, Phys Rev Lett 63, 1499 (1989)

${ }^{303} \mathrm{~N}$ Garcla and H Rohrer, $J$ Phys Condens Matter 1, 3737 (1989)

${ }^{304} \mathrm{R}$ Trzcinsk1, E Gmelın, and H J Queisser, Phys Rev B 35, 6373 (1987)

${ }^{305}$ A G M Jansen, A P van Gelder, and P Wyder, J Phys C 13, 6073 (1980)
} 


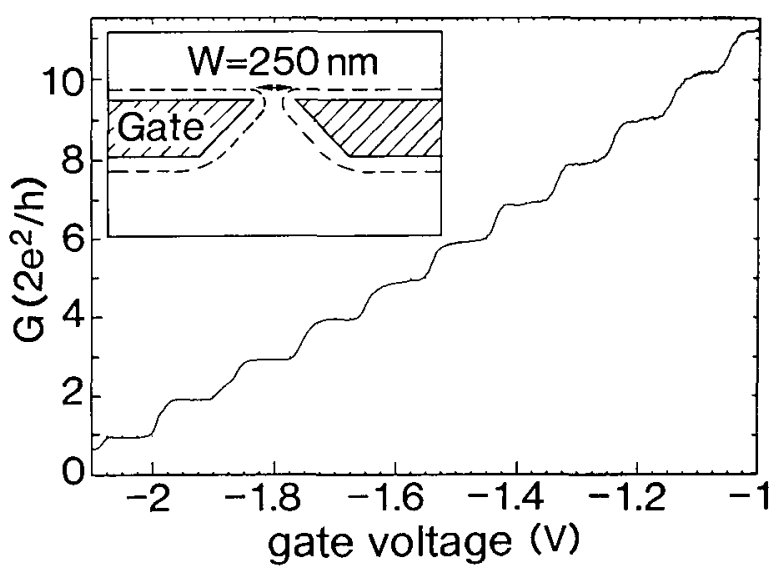

Fig. 44. Point contact conductance as a function of gate voltage at $0.6 \mathrm{~K}$, demonstrating the conductance quantization in units of $2 e^{2} / h$. The data are obtained from the two-terminal resistance after subtraction of a background resistance. The constriction width increases with increasing voltage on the gate (see inset). Taken from B. J. van Wees et al., Phys. Rev. Lett. 60, 848 (1988).

explanation of this effect relies on the fact that each 1D subband in the constriction contributes $2 e^{2} / h$ to the conductance because of the cancellation of the group velocity and the 1D density of states discussed in Section 12. Since the number $N$ of occupied subbands is necessarily an integer, it follows from this simple argument that the conductance $G$ is quantized,

$$
G=\left(2 e^{2} / h\right) N,
$$

as observed experimentally. A more complete explanation requires an explicit treatment of the mode coupling at the entrance and exit of the constriction, as discussed later.

The zero-field conductance quantization of a quantum point contact is not as accurate as the Hall conductance quantization in strong magnetic fields. The deviations from exact quantization are typically $6,7,3061 \%$, while in the quantum Hall effect one obtains routinely ${ }^{97}$ an accuracy of 1 part in $10^{7}$. It is unlikely that a similar accuracy will be achieved in the case of the zero-field quantization, one reason being the additive contribution to the point contact resistance of a background resistance whose magnitude cannot be determined precisely. The largest part of this background resistance originates in the ohmic contacts ${ }^{307}$ and can thus be eliminated in a four-terminal measurement of the contact resistance. The position of the additional voltage

${ }^{306}$ G. Timp, R. Behringer, S. Sampere, J. E. Cunningham, and R. E. Howard, in Ref. 15; see also

G. Timp in Ref. 9.

${ }^{307}$ H. van Houten, C. W. J. Beenakker, and B. J. van Wees, in Ref. 9. 
probes on the wide 2DEG regions has to be more than an inelastic scattering length away from the point contact so that a local equilibrium is established A residual background resistance ${ }^{307}$ of the order of the resistance $\rho$ of a square is therefore unavoldable In the experıments of Refs 6 and 7 one has $\rho \approx 20 \Omega$, but lower values are possible for higher-mobility material It would be of interest to investigate experimentally whether resistance plateaux quantized to such an accuracy are achievable It should be noted, however, that the degree of flatness of the plateaux and the sharpness of the steps in the present experiments vary among devices of identical design, indicatıng that the detailed shape of the electrostatic potential defining the constriction is important There are many uncontrolled factors affecting this shape, such as small changes in the gate geometry, variations in the pinning of the Fermi level at the free GaAs surface or at the interface with the gate metal, doping inhomogeneities in the heterostructure material, and trapping of charge in deep levels in AlGaAs

On increasing the temperature, one finds experimentally that the plateaux acquire a finite slope until they are no longer resolved ${ }^{308}$ This is a consequence of the thermal smearing of the Fermi-Dirac distribution (49) If at $T=0$ the conductance $G\left(E_{\mathrm{F}}, T\right)$ has a step function dependence on the Fermi energy $E_{F}$, at finite temperatures it has the form ${ }^{309}$

$$
G\left(E_{\mathrm{F}}, T\right)=\int_{0}^{\infty} G(E, 0) \frac{d f}{d E_{\mathrm{F}}} d E=\frac{2 e^{2}}{h} \sum_{n=1}^{\infty} f\left(E_{n}-E_{\mathrm{F}}\right)
$$

Here $E_{n}$ denotes the energy of the bottom of the $n$th subband [cf Eq (43)] The width of the thermal smearing function $d f / d E_{\mathrm{F}}$ is about $4 k_{\mathrm{B}} T$, so the conductance steps should disappear for $T \gtrsim \Delta E / 4 k_{B} \sim 4 \mathrm{~K}$ (here $\Delta E$ is the subband splittıng at the Fermi level) This is confirmed both by exper1ment $^{308}$ and by numerical calculations (see below)

Interestingly, it was found experimentally ${ }^{7}$ that in general a finite temperature yielded the most pronounced and flat plateaux as a function of gate voltage in the zero-field conductance If the temperature is increased beyond this optımum (which is about $05 \mathrm{~K}$ ), the plateaux disappear because of the thermal averaging discussed earlier Below this temperature, an oscllatory structure may be superımposed on the conductance plateaux This phenomenon depends on the precise shape of the constriction, as discussed later A small but finte voltage drop across the constiction has an effect that is qualitatively similar to that of a finite temperature ${ }^{309}$ This is indeed borne out by experiment ${ }^{308}$ (Experiments on conduction through quantum point

${ }^{308}$ B J van Wees, L P Kouwenhoven, E M M Willems, C J P M Harmans, J E Moolj, H van Houten, $\mathrm{C}$ W J Beenakker, $\mathbf{J} \mathrm{G}$ Willamson and $\mathrm{C} \mathrm{T}$ Foxon, submitted to Phys $\operatorname{Rev} B$

${ }^{309}$ P F Bagwell and T P Orlando, Phys Rev B 40, 1456 (1989) 
(a)

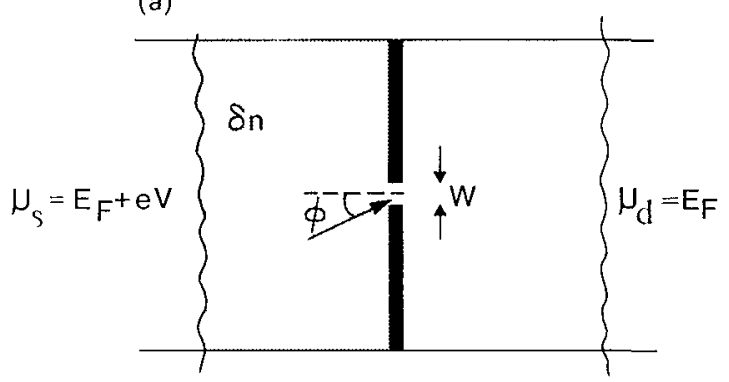

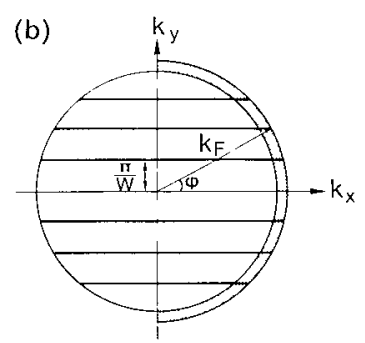

Fig 45 (a) Classical ballistic transport through a point contact induced by a concentration difference $\delta n$, or electrochemical potential difference $e V$, between source (s) and drain (d). (b) The net current through a quantum point contact is carried by the shaded region in $k$-space. In a narrow channel the allowed states lie on the horizontal lines, which correspond to quantized values for $k_{y}= \pm n \pi / W$, and continuous values for $k_{x}$ The formation of these 1D subbands gives rise to a quantized conductance. Taken from $\mathrm{H}$ van Houten et al, in "Physics and Technology of Submicron Structures" (H Heinrıch, G Bauer, and F Kuchar, eds) Springer, Berlın, 1988, in "Nanostructure Physics and Fabrication" (M Reed and W P. Kırk, eds) Academıc, New York, 1989

contacts at larger applied voltages in the nonlinear transport regime have been reviewed in Ref. 307).

Theoretically, one would expect the conductance quantization to be preserved in longer channels than those used in the original experiments $s^{6,7}$ (in which typically $L \sim W \sim 100 \mathrm{~nm}$ ). Experiments on channels longer than about $1 \mu \mathrm{m}$ did not show the quantization, ${ }^{306,307,310}$ however, although their length was well below the transport mean free path in the bulk (about $10 \mu \mathrm{m}$ ). The lack of clear plateaux in long constrictions is presumably due to enhanced backscattering inside the constriction, either because of impurity scattering (which may be enhanced ${ }^{306,310}$ due to the reduced screening in a quasi-one-dimensional electron gas $^{72}$ ) or because of boundary scattering at channel wall irregularities. As mentioned in Section 5, Thornton et al. ${ }^{107}$ have found evidence for a small $(5 \%)$ fraction of diffuse, rather than specular, reflections at boundaries defined electrostatically by a gate. In a 200-nm-wide constriction this leads to an effective mean free path of about $200 \mathrm{~nm} / 0.05 \approx 4 \mu \mathrm{m}$, comparable to the constriction length of devices that do not exhibit the conductance quantization. ${ }^{113.307}$

(2) Theory. It is instructive to first consider classical 2D point contacts in some detail. ${ }^{31,311}$ The ballistic electron flow through a point contact is illustrated in Fig. 45a in real space, and in Fig. 45b in $k$-space, for a small

${ }^{310} \mathrm{G}$ Timp, in Ref 10

${ }^{311} \mathrm{H}$ van Houten and C W J Beenakker, in Ref 15 
excess electron density $\delta n$ at one side of the point contact. At low temperatures this excess charge moves with the Fermi velocity $v_{F}$. The flux normally incident on the point contact is $\delta n v_{\mathrm{F}}\langle\cos \phi \theta(\cos \phi)\rangle$, where $\theta(x)$ is the unit step function and the symbol $\langle>$ denotes an isotropic angular average (the angle $\phi$ is defined in Fig. 45a). In the ballistic limit $l \gg W$ the incident flux is fully transmitted, so the total diffusion current $J$ through the point contact is given by

$$
J=W \delta n v_{\mathrm{F}} \int_{-\pi / 2}^{\pi / 2} \cos \phi \frac{d \phi}{2 \pi}=\frac{1}{\pi} W v_{\mathrm{F}} \delta n .
$$

The diffusance $\tilde{D} \equiv J / \delta n=(1 / \pi) W v_{F} ;$ therefore, the conductance $G=$ $e^{2} \rho\left(E_{\mathrm{F}}\right) \tilde{D}$ becomes (using the $2 \mathrm{D}$ density of states (4.2) with the appropriate degeneracy factors $g_{s}=2, g_{v}=1$ )

$$
G=\frac{2 e^{2}}{h} \frac{k_{\mathrm{F}} W}{\pi}, \quad \text { in } 2 \mathrm{D} .
$$

Eq. (13.4) is the $2 \mathrm{D}$ analogue ${ }^{6}$ of Sharvin's well-known expression ${ }^{296}$ for the point contact conductance in three dimensions,

$$
G=\frac{2 e^{2}}{h} \frac{k_{\mathrm{F}}^{2} S}{4 \pi}, \quad \text { in } 3 \mathrm{D},
$$

where now $S$ is the area of the point contact. The number of propagating modes for a square-well lateral confining potential is $N=\operatorname{Int}\left[k_{\mathrm{F}} W / \pi\right]$ in $2 \mathrm{D}$, so Eq. (13.4) is indeed the classical limit of the quantized conductance (13.1).

Quantum mechanically, the current through the point contact is equipartitioned among the $1 \mathrm{D}$ subbands, or transverse modes, in the constriction. The equipartitioning of current, which is the basic mechanism for the conductance quantization, is illustrated in Fig. 45b for a square-well lateral confining potential of width $W$. The 1D subbands then correspond to the pairs of horizontal lines at $k_{y}= \pm n \pi / W$, with $n=1,2, \ldots, N$ and $N=\operatorname{Int}\left[k_{\mathrm{F}} W / \pi\right]$. The group velocity $v_{n}=\hbar k_{x} / m$ is proportional to $\cos \phi$ and thus decreases with increasing $n$. However, the decrease in $v_{n}$ is compensated by an increase in the $1 \mathrm{D}$ density of states. Since $\rho_{n}$ is proportional to the length of the horizontal lines within the dashed area in Fig. $45 \mathrm{~b}, \rho_{n}$ is proportional to $1 / \cos \phi$ so that the product $v_{n} \rho_{n}$ does not depend on the subband index. We emphasize that, although the classical formula (13.4) holds only for a squarewell lateral confining potential, the quantization (13.1) is a general result for any shape of the confining potential. The reason is simply that the fundamental cancellation of the group velocity $v_{n}=d E_{n}(k) / \hbar d k$ and the $1 \mathrm{D}$ density of states $\rho_{n}^{+}=\left(\pi d E_{n}(k) / d k\right)^{-1}$ holds regardless of the form of the dispersion relation $E_{n}(k)$. For the same reason, Eq. (13.1) is equally applicable 
in the presence of a magnetıc field, when magnetıc edge channels at the Ferm level take over the role of $1 \mathrm{D}$ subbands Equation (131) thus implies a continuous transition from the zero-field quantization to the quantum Hall effect, as we will discuss in Section $13 \mathrm{~b}$

To analyze deviations from Eq (131) it is necessary to solve the Schrodinger equation for the wave functions in the narrow point contact and the adjacent wide regions and to match the wave functions and their derivatives at the entrance and exit of the constriction The resulting transmission coefficients determine the conductance via the Landauer formula (12 10) This mode coupling problem has been solved numerically for point contacts of a variety of shapes ${ }^{312-321}$ and analytically in special geometries ${ }^{322-324}$ When considering the mode coupling at the entrance and exit of the constriction, one must distinguish gradual (adlabatıc) from abrupt transitions from wide to narrow regions

The case of an adiabatic constriction has been studied by Glazman et $a l,{ }^{325}$ by Yacoby and Imry ${ }^{326}$ and by Payne ${ }^{272}$ If the constriction width $W(x)$ changes sufficiently gradually, the transport through the constriction is adiabatic ( $1 \mathrm{e}$, without intersubband scattering) The transmission coefficients then vanish, $\left|t_{n m}\right|^{2}=0$, unless $n=m \leqslant N_{\text {min }}$, with $N_{\text {min }}$ the smallest number of occupied subbands in the constriction The conductance quantization (13 1) now follows immediately from the Landauer formula (12 10) The criterion for adiabatic transport $1 \mathrm{~s}^{326} d W / d x \leqq 1 / N(x)$, with $N(x) \approx k_{\mathrm{F}} W(x) / \pi$ the local number of subbands As the constriction widens, $N(x)$ increases and adiabaticity is preserved only if $W(x)$ increases more and more slowly In practice, adiabaticity breaks down at a width $W_{\max }$, which is at most a factor of 2 larger than the minımum width $W_{\text {mın }}$ (cf the collımated beam experiment of Ref

\footnotetext{
${ }^{312}$ L Escapa and N Garcla, $J$ Phys Condens Matter 1, 2125 (1989)

${ }^{313}$ E G Haanappel and D van der Marel, Phys Rev B 39, 5484 (1989), D van der Marel and E

G Haanappel, Phys Rev B 39, 7811 (1989)

${ }^{314} \mathrm{G}$ Kirczenow, Solld State Comm 68, 715 (1988), J Phys Condens Matter 1, 305 (1989)

${ }^{315}$ A Szafer and A D Stone, Phys Rev Lett 62, 300 (1989)

${ }^{316}$ E Tekman and S Ciraci, Phys Rev B 39, 8772 (1989), Phys Rev B 40, 8559 (1989)

${ }^{317}$ Song He and S Das Sarma, Phys Rev B 40, 3379 (1989)

${ }^{318} \mathrm{D}$ van der Marel, in Ref 15

${ }^{319} \mathrm{~N}$ Garcia and L Fscapa, Appl Phys Lett 54, 1418 (1989)

${ }^{320}$ E Castaño and G Kurczenow, Soltd State Comm 70, 801 (1989)

${ }^{321} \mathrm{Y}$ Avishai and Y B Band, Phys Rev B 40, 12535 (1989)

${ }^{322}$ A Kawabata, $J$ Phys Soc Japan 58, $372(1989)$

${ }^{323}$ I B Levinson, Pts'ma Zh Eksp Teor $F_{l z}$ 48, 273 (1988) [JETP Lett 48, 301 (1988)]

${ }^{324}$ A Matulis and D Segzda, $J$ Phys Condens Matter 1, 2289 (1989)

${ }^{325}$ L I Glazman, G B Lesovick, D E Khmel'nitskı1, R I Shekhter, Pls'ma Zh Teor Fiz 48, 218 (1988) [JETP Lett 48, 238 (1988)]

${ }^{326}$ A Yacoby and Y Imry, Phys Rev B 415341 (1990)
} 
327, discussed in Section 15) This does not affect the conductance of the constriction, however, if the breakdown of adiabaticity results in a mixing of the subbands without causing reflection back through the constriction If such is the case, the total transmission probability through the constriction remains the same as in the hypothetical case of fully adiabatic transport As pointed out by Yacoby and Imry, ${ }^{326}$ a relatively small adiabatic increase in width from $W_{\min }$ to $W_{\max }$ is sufficient to ensure a drastic suppression of reflections at $W_{\mathrm{m} \text { ix }}$ The reason is that the subbands with the largest reflection probability are close to cutoff, that 1s, they have subband index close to $N_{\max }$, the number of subbands occupied at $W_{\mathrm{m} \text { ix }}$ Because the transport is adiabatic from $W_{\min }$ to $W_{\max }$, only the $N_{\min }$ subbands with the smallest $n$ arrive at $W_{\mathrm{m} \mathrm{x}}$, and these subbands have a small reflection probability In the language of waveguide transmission, one has impedance-matched the constriction to the wide 2 DEG regions ${ }^{328}$ The filtering of subbands by a gradually widening constriction has an interesting effect on the angular distribution of the electrons injected into the wide 2DEG This horn collumation effect ${ }^{329}$ is discussed in Section 15

An adiabatic constriction improves the accuracy of the conductance quantization, but is not required to observe the effect Calculations ${ }^{312} 324$ show that well-defined conductance plateaux persist for abrupt constrictions, especially if they are neither very short nor very long The optımum length for the observation of the plateaux is given by ${ }^{313} L_{\text {opt }} \approx 04\left(W \lambda_{\mathrm{F}}\right)^{1 / 2}$ In shorter constrictions the plateaux acquire a finite slope, although they do not disappear completely even at zero length For $L>L_{\mathrm{opt}}$ the calculations exhibit regular oscillations that depress the conductance periodically below its quantized value The oscillations are damped and have usually vanished before the next plateau is reached As a representative illustration, we reproduce in Fig 46 a set of numerical results for the conductance as a function of width (at fixed Fermı wave vector), obtained by Szafer and Stone ${ }^{315}$ Note that a finite temperature improves the flatness of the plateaux, as observed experımentally The existence of an optımum length can be understood as follows

Because of the abrupt widening of the constriction, there is a significant probability for reflection at the exit of the constriction, in contrast to the adrabatic case considered earlier The conductance as a function of width, or Fermi energy, is therefore not a simple step function On the $n$th conductance plateau backscattering occurs predominantly for the $n$th subband, since it is closest to cutoff Resonant transmission of this subband occurs if

\footnotetext{
${ }^{327}$ L W Molenkamp, A A M Starıng, C W J Beenakker, R Eppenga, C E Timmering, J G Willamson, C J P M Harmans, and C T Foxon, Phys Rev B 41, 1274 (1990)

${ }^{328} \mathrm{R}$ Landauer, $Z$ Phys $B$ 68, 217 (1987)

${ }^{329} \mathrm{C}$ W J Beenakker and H van Houten, Phys Rev B 39, 10445 (1989)
} 


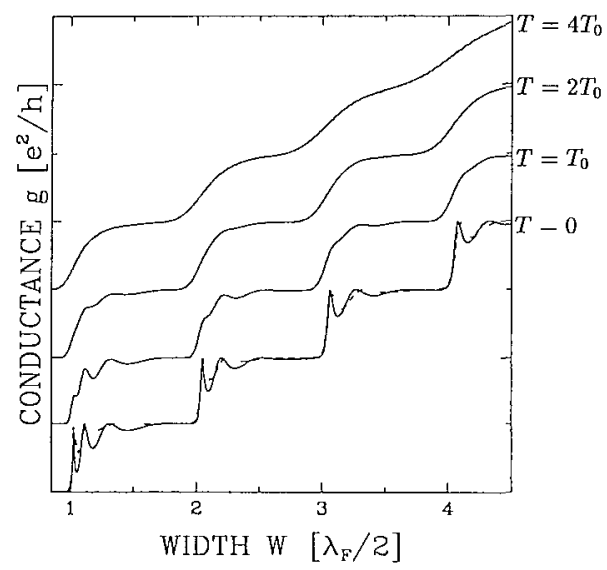

Fig 46 Transmission resonances exhibited by theoretical results for the conductance of a quantum point contact of abrupt (rectangular) shape $\mathrm{A}$ smearnng of the resonances occurs at nonzero temperatures $\left(T_{0}=002 E_{\mathrm{F}} / k_{\mathrm{B}} \approx 28 \mathrm{~K}\right)$ The dashed curve is an exact numerical result, the full curves are approximate Taken from A Szafer and A D Stone, Phys Rev Lett 62, 300 (1989)

the constriction length $L$ is approximately an integer multiple of half the longitudinal wavelength $\lambda_{n}=h\left[2 m\left(E_{\mathrm{F}}-E_{n}\right)\right]^{-1 / 2}$, leading to oscillations on the conductance plateaux These transmission resonances are damped, because the reflection probability decreases with decreasing $\lambda_{n}$ The shortest value of $\lambda_{n}$ on the $N$ th conductance plateau is $h\left[2 m\left(E_{N+1}-E_{N}\right)\right]^{1 / 2} \approx\left(W \lambda_{\mathrm{F}}\right)^{1 / 2}$ (for a square-well lateral confining potent1al) The transmission resonances are thus suppressed if $L \lesssim\left(W \lambda_{\mathrm{F}}\right)^{1 / 2}$ Transmission through evanescent modes (1 e, subbands above $E_{\mathrm{F}}$ ) is predominant for the $(N+1)$ th subband, since it has the largest decay length $\Lambda_{N+1}=h\left[2 m\left(E_{N+1}-E_{\mathrm{F}}\right)\right]^{1 / 2}$ The observation of that plateau requires that the constriction length exceeds this decay length at the population threshold of the $N$ th mode, or $L \gtrsim h\left[2 m\left(E_{N+1}-E_{N}\right)\right]^{1 / 2} \approx\left(W \lambda_{\mathrm{F}}\right)^{1 / 2}$ The optimum length $^{313} L_{\mathrm{opt}} \approx 04\left(W \lambda_{\mathrm{F}}\right)^{1 / 2}$ thus separates a short constriction regime, in which transmission via evanescent modes cannot be ignored, from a long constriction regime, in which transmission resonances obscure the plateaux

Oscillatory structure was resolved in low-temperature experıments on the conductance quantization of one quantum point contact by van Wees et al, ${ }^{308}$ but was not clearly seen in other devices A difficulty in the interpretation of these and other experiments is that oscillations can also be caused by quantum interference processes involving impurity scattering near the constriction Another experimental observation of oscillatory structure 


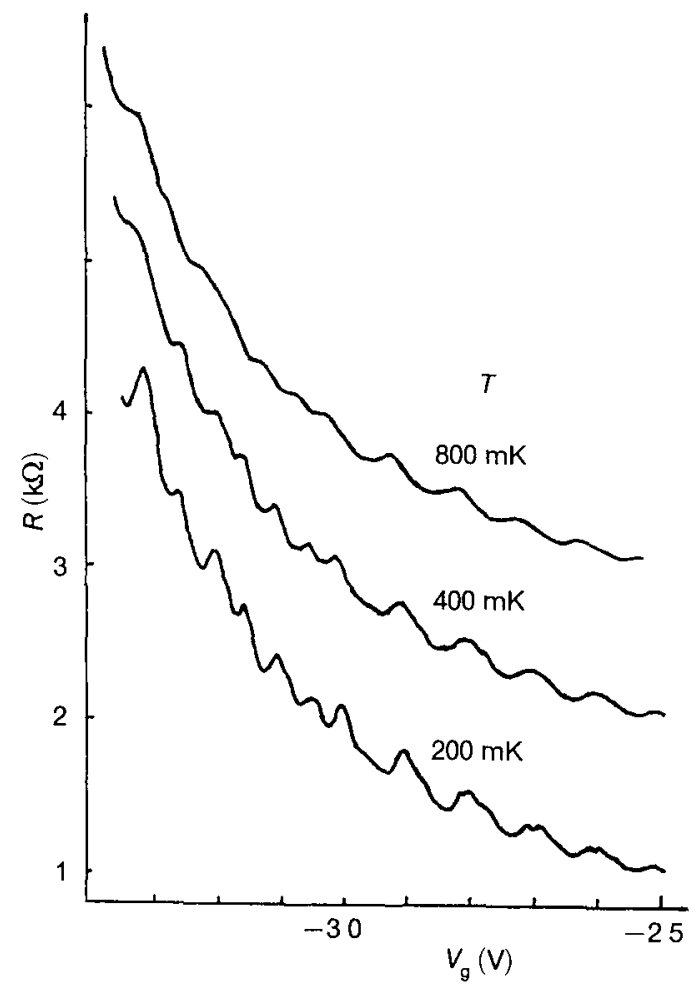

FIG 47 Resistance as a function of gate voltage for an elongated quantum point contact $(L=08 \mu \mathrm{m}$ ) at temperatures of 02,04 , and $08 \mathrm{~K}$, showing transmission resonances Subsequent curves from the bottom are offset by $1 \mathrm{k} \Omega$ Taken from R J Brown et al, Sold State Electron 32, 1179 (1989)

was reported by Hirayama et al $^{330}$ for short (100-nm) quantum point contacts of fixed width (defined by means of focused ion beam lithography) To observe the plateaux, they slowly varied the electron density by weakly illuminatıng the sample The oscillations were quite reproducible, also after thermal cycling of the sample, but again they were found in some of the devices only (this was attributed to variations in the abruptness of the constrictions ${ }^{330331}$ ) Brown et al ${ }^{332}$ have studied the conductance of splitgate constrictions of lengths $L \approx 03,08$, and $1 \mu \mathrm{m}$, and they observed pronounced oscillations instead of the flat conductance plateaux found for shorter quantum point contacts The observed oscllatory structure (reproduced in Fig 47) is quite regular, and it correlates with the sequence of ${ }^{330}{ }^{\mathrm{Y}}$ Hirayama, T Saku, and Y Horkoshi, Phys Rev B 39, 5535 (1989)

${ }^{331}$ Y Hirayama, T Saku, and Y Horkoshi, Jap J Appl Phys 28, L701 (1989)

${ }^{332}$ R J Brown, M J Kelly, R Newbury, M Pepper, B Miller, H Ahmed, D G Hasko, D C

Peacock, D A Ritchie, J E F Frost, and G A C Jones, Solid State Electron 32, 1179 (1989) 
plateaux that is recovered at higher temperatures (around $08 \mathrm{~K}$ ) The effect was seen in all of the devices studied in Ref 332 Measurements by Timp et $a^{306}$ on rather similar 09- $\mu$ m-long constrictions did not show periodic oscillations, however Brown et al conclude that their oscillations are due to transmission resonances associated with reflections at entrance and exit of the constriction Detailed comparison with theory is difficult because the transmission resonances depend sensitively on the shape of the lateral confining potential and on the presence of a potential barrier in the constriction (see Section $13 \mathrm{~b}$ ) A calculation that comes close to the observation of Brown et al has been published by Martin-Moreno and Smith ${ }^{333}$

b Depopulation of Subbands and Suppression of Backscattering by a Magnetic Field

The effect of a magnetic field (perpendicular to the 2DEG) on the quantized conductance of a point contact is shown in Fig 48, as measured by van Wees et al ${ }^{334}$ First of all, Fig 48 demonstrates that the conductance quantization is conserved in the presence of a magnetic field and shows a smooth transition from zero-field quantization to quantum Hall effect The most noticeable effect of the magnetic field is to reduce the number of plateaux in a given gate voltage interval This provides a demonstration of depopulation of magnetoelectric subbands, which is more direct than that provided by the experiments discussed in Section 10 In addition, one observes that the flatness of the plateaux improves in the presence of the field This is due to the reduction of the reflection probability at the point contact, which is revealed most clearly in a somewhat different (four-terminal) measurement configuration These two effects of a magnetic field will be discussed separately We will return to the magnetic suppression of backscattering in Section 18 in connection with the edge channel theory ${ }^{112}$ of the quantum Hall effect

(1) Depopulation of Subbands. Because the equipartitioning of current among the $1 \mathrm{D}$ subbands holds regardless of the nature of the subbands involved, one can conclude that in the presence of a magnetic field $B$ the conductance remains quantized according to $G=\left(2 e^{2} / h\right) N$ (1gnoring spin spliting of the subbands, for simplicity) Explicit calculations ${ }^{335}$ confirm this expectation The number of occupied subbands $N$ as a function of $B$ has been

${ }^{333}$ L Martın-Moreno and C G Smith, $J$ Phys Condens Matter 1, 5421 (1989)

${ }^{334}$ B J van Wees, L P Kouwenhoven, $H$ van Houten, C W J Beenakker, J E Moo1], C T Foxon, and J J Harris, Phys Rev B 38, 3625 (1988)

${ }^{335}$ M Buttıker Phys Rev B 417906 (1990), L I Glazman and A V Khaetsk11, J Phys Condens Matter 1, 5005 (1989) Y Avishat and Y B Band, Phys Rev B 40, 3429 (1989), K B Efetov, $J$ Phys Condens Matter 1, 5535 (1989) 


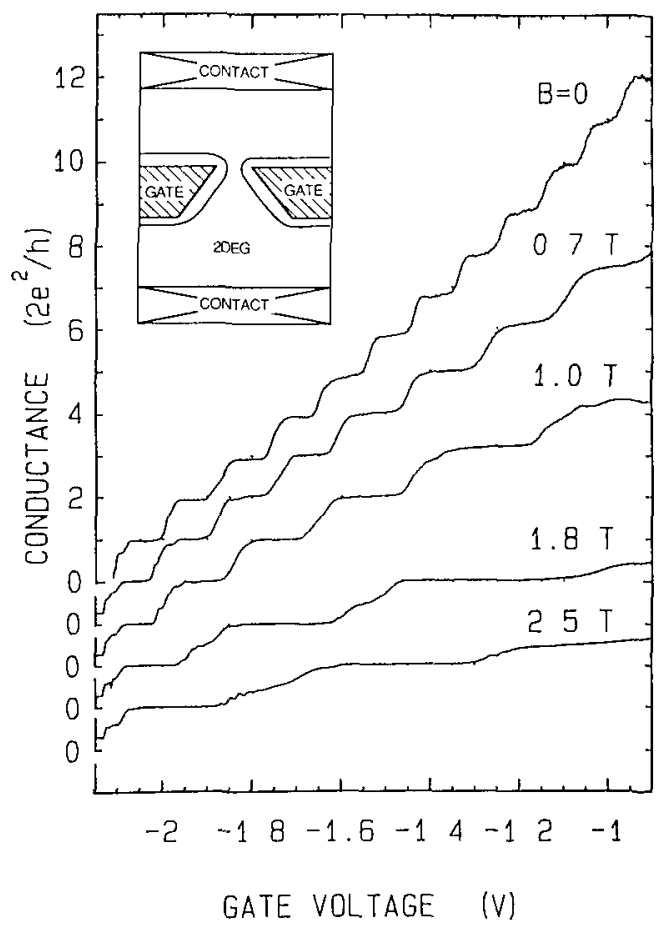

Fig. 48. Point contact conductance (corrected for a background resistance) as a function of gate voltage for several magnetic field values, illustrating the transition from zero-field quantization to quantum Hall effect. The curves have been offset for clarity. The inset shows the device geometry. Taken from B. J. van Wees et al., Phys. Rev. B. 38, 3625 (1988).

studied in Sections 10 and 12 and is given by Eqs. (10.7) and (10.8) for a parabolic and a square-well potential, respectively. In the high-magnetic-field regime $W \gtrsim 2 l_{\text {cycl }}$, the number $N \approx E_{\mathrm{F}} / \hbar \omega_{\mathrm{c}}$ is just the number of occupied Landau levels. The conductance quantization is then a manifestation of the quantum Hall effect. ${ }^{8}$ (The fact that $G$ is not a Hall conductance but a twoterminal conductance is not an essential distinction for this effect; see Section 18.) At lower magnetic fields, the conductance quantization provides a direct and extremely straightforward method to measure via $N=G\left(2 e^{2} / h\right)^{-1}$ the depopulation of magnetoelectric subbands in the constriction.

Figure 49 shows $N$ versus $B^{-1}$ for various gate voltages, as it follows from the experiment of Fig. 48. Also shown are the theoretical curves for a squarewell confining potential, with the potential barrier in the constriction taken into account by replacing $E_{\mathrm{F}}$ by $E_{\mathrm{F}}-E_{\mathrm{c}}$ in Eq. (10.8). The $B$-dependence of $E_{\mathrm{F}}$ has been ignored in the calculation. The barrier height $E_{\mathrm{c}}$ is obtained from the high-field conductance plateaux [where $\left.N \approx\left(E_{\mathrm{F}}-E_{\mathrm{c}}\right) / \hbar \omega_{\mathrm{c}}\right]$, and the 


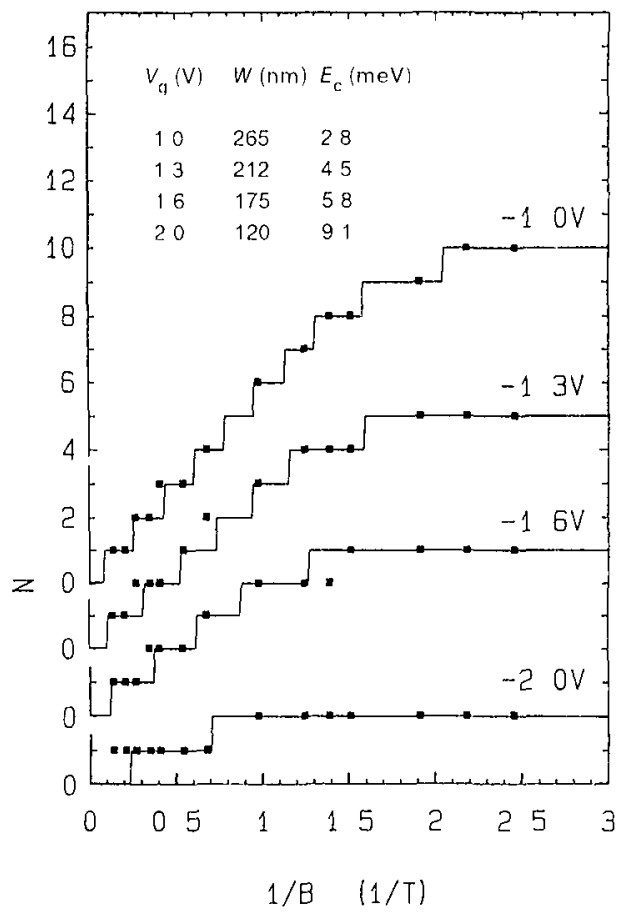

FIG 49 Number of occupied subbands as a function of reciprocal magnetic field for several values of the gate voltage Data points have been obtained directly from the quantized conductance ( $\left.F_{1 g} 48\right)$, solid curves are calculated for a square-well confining potentral of width $W$ and well bottom $E_{\mathrm{c}}$ as tabulated in the inset Taken from $\mathbf{B} \mathbf{J}$ van Wees et al, Phys Rev B 38, 3625 (1988)

constriction width $W$ then follows from the zero-field conductance (where $\left.N \approx\left[2 m\left(E_{\mathrm{F}}-E_{\mathrm{c}}\right) / \hbar^{2}\right]^{1 / 2} W / \pi\right)$ The good agreement found over the entire field range confirms the expectation that the quantized conductance is exclusively determined by the number of occupied subbands, irrespective of their electric or magnetic origin The analysis in Fig 49 is for a square-well confining potentral ${ }^{334}$ For the narrowest constrictions a parabolic potential should be more approprate, ${ }^{61}$ which has been used to analyze the data of Fig 48 in Refs 336 and 308 Wharam et al ${ }^{337}$ have analyzed their depopulation data using the intermediate model of a parabolic potential with a flattened bottom (cf also Ref 336) Because of the uncertainties in the

${ }^{336}$ J F Welsz and K -F Berggren, Phys Rev B 40, 1325 (1989)

${ }^{337}$ D A Wharam, U Ekenberg, M Pepper, D G Hasko, H Ahmed, J E F Frost, D A Ritchie,

D C Peacock, and G A C Jones, Phys Rev B 39,6283 (1989) 
actual shape of the potential, the parameter values tabulated in Fig. 49 should be considered as rough estimates only.

In strong magnetic fields the spin degeneracy of the energy levels is removed, and additional plateaux appear ${ }^{7,334}$ at odd multiples of $e^{2} / h$. Wharam et al. ${ }^{7}$ have demonstrated this effect in a particularly clear fashion, using a magnetic field parallel (rather than perpendicular) to the 2DEG. Rather strong magnetic fields turned out to be required to fully lift the spin degeneracy in this experiment (about 10 T).

(2) Suppression of Backscattering. Only a small fraction of the electrons injected by the current source into the $2 \mathrm{DEG}$ is transmitted through the point contact. The remaining electrons are scattered back into the source contact. This is the origin of the nonzero resistance of a ballistic point contact. In this subsection we shall discuss how a relatively weak magnetic field leads to a suppression of the geometrical backscattering caused by the finite width of the point contact, while the amount of backscattering caused by the potential barrier in the point contact remains essentially unaffected.

The reduction of backscattering by a magnetic field is observed as a negative magnetoresistance [i.e., $R(B)-R(0)<0$ ] in a four-terminal measurement of the longitudinal point contact resistance $R_{\mathrm{L}}$. The voltage probes in this experiment ${ }^{113}$ are positioned on wide $2 D E G$ regions, well away from the constriction (see the inset in Fig. 50). This allows the establishment of local equilibrium near the voltage probes, at least in weak magnetic fields (cf. Sections 18 and 19), so that the measured four-terminal resistance does not depend on the properties of the probes. The experimental results for $R_{\mathrm{L}}$ in this geometry are plotted in Fig. 50. The negative magnetoresistance is temperature-independent (between $50 \mathrm{mK}$ and $4 \mathrm{~K}$ ) and is observed in weak magnetic fields once the narrow constriction is defined (for $V_{\mathrm{g}} \lesssim-0.3 \mathrm{~V}$ ). At stronger magnetic fields $(B>0.4 \mathrm{~T})$, a crossover is observed to a positive magnetoresistance. The zero-field resistance, the magnitude of the negative magnetoresistance, the slope of the positive magnetoresistance, as well as the crossover field, all increase with increasing negative gate voltage.

The magnetic field dependence of the four-terminal resistance shown in Fig. 50 is qualitatively different from that of the two-terminal resistance $R_{2 \mathrm{t}} \equiv G^{-1}$ considered in the previous subsection. In fact, $R_{2 \mathrm{t}}$ is approximately $B$-independent in weak magnetic fields (below the crossover fields of Fig. 50). The reason is that $R_{21}$ is given by [cf. Eq. (13.1)]

$$
R_{21}=\frac{h}{2 e^{2}} \frac{1}{N_{\min }},
$$

with $N_{\min }$ the number of occupied subbands in the constriction (at the point where it has its minimum width and electron gas density). In weak magnetic fields such that $2 l_{\text {cycl }}>W$, the number of occupied subbands remains 


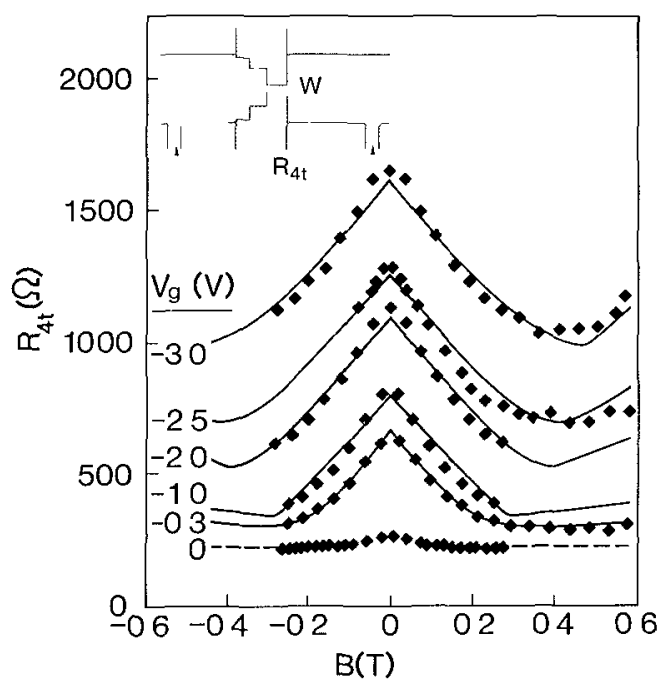

FIG 50 Four-termınal longitudinal magnetoresistance $R_{\mathrm{L}}$ of a constriction for a series of gate voltages The negative magnetoresistance is temperature independent between $50 \mathrm{mK}$ and $4 \mathrm{~K}$ Solid lines are according to Eqs (137) and (108), with the constriction width as adjustable parameter The inset shows schematically the device geometry, with the two voltage probes used to measure $R_{\mathbf{L}}$ Taken from $\mathrm{H}$ van Houten et al, Phys Rev B 37, 8534 (1988)

approximately constant [cf $F_{1 g} 31$ or Eq (108)], so $R_{2 t}$ is only weakly dependent on $B$ in this field regime For stronger fields Eq (136) describes a positive magnetoresistance, because $N_{\min }$ decreases due to the magnetic depopulation of subbands discussed earlier (A similar positive magnetoresistance is found in a Hall bar with a cross gate, see Ref 338 ) Why then does one find a negative magnetoresistance in the four-terminal measurements of Fig $50^{7}$ Qualitatively, the answer is shown in Fig 51, for a constriction without a potential barrier In a magnetic field the left- and right-moving electrons are spatially separated by the Lorentz force at opposite sides of the constriction Quantum mechanically the skipping orbits in Fig 51 correspond to magnetic edge states ( $\mathrm{cf} \mathrm{F}_{1 \mathrm{~g}}$ 41) Backscattering thus requires scattering across the width of the constriction, which becomes increasingly improbable as $l_{\text {cycl }}$ becomes smaller and smaller compared with the width (compare Figs $51 \mathrm{a}, \mathrm{b}$ ) For this reason a magnetic field suppresses the geometrical constriction resistance in the ballistic regime, but not the resistance associated with the constriction in energy space, which is due to the potential barrier

These effects were analyzed theoretically in Ref 113 , with the simple result

$$
R_{\mathrm{L}}=\frac{h}{2 e^{2}}\left(\frac{1}{N_{\min }}-\frac{1}{N_{\text {wide }}}\right)
$$

${ }^{338}$ H Hiral, S Komıama, S Sasa and T Fuj11, Solhd State Comm 72, 1033 (1989) 
a)

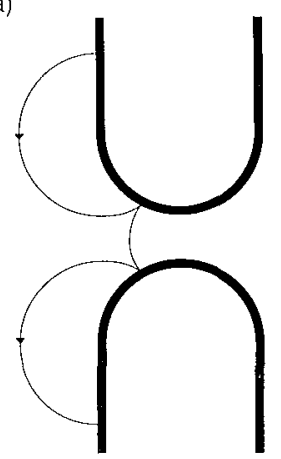

b)

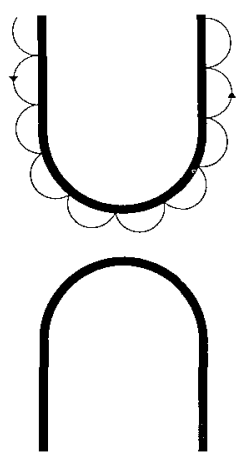

FIG 51 Illustration of the reduction of backscattering by a magnetic field, which is responsible for the negative magnetoresistance of Fig 50 Shown are trajectories approaching a constriction without a potential barrier in a weak (a) and strong (b) magnetic field Taken from $\mathrm{H}$ van Houten $e t$ al in 'Nanostructured Systems (M A Reed ed) Academic New York

Here $N_{\text {wide }}$ is the number of occupied Landau levels in the wide 2DEG regions The simplest (but incomplete) argument leading to Eq (13 7) is that the additivity of voltages on reservorrs (ohmic contacts) implies that the twotermınal resistance $R_{21}=\left(h / 2 e^{2}\right) N_{\min }^{1}$ should equal the sum of the Hall resistance $R_{\mathrm{H}}=\left(h / 2 e^{2}\right) N_{\text {wide }}^{-1}$ and the longitudinal resistance $R_{\mathrm{L}}$ This argument is incomplete because it assumes that the Hall resistance in the wide regions is not affected by the presence of the constriction This is correct in general only if inelastıc scattering has equilıbrated the edge states transmitted through the constriction before they reach a voltage probe Deviations from Eq (137) can occur in the absence of local equilibrium near the voltage probes, depending on the properties of the probes themselves We discuss this in Section 19, following a derivation of $\mathrm{Eq}$ (137) from the LandauerButtiker formalism ${ }^{112}$

At small magnetic fields $N_{\min }$ is approximately constant, while $N_{\text {wide }} \approx E_{\mathrm{r}} / h \omega_{\mathrm{c}}$ decreases linearly with $B$ Equation (137) thus predicts a negative magnetoresistance If the electron density in the wide and narrow regions is equal ( $1 \mathrm{e}$, the barrier height $E_{\mathrm{c}}=0$ ), then the resistance $R_{\mathrm{L}}$ vanishes for fields $B>B_{\text {crit }} \equiv 2 \hbar k_{\mathrm{F}} / e W$ This follows from Eq (137), because in this case $N_{\min }$ and $N_{\text {widc }}$ are identical If the electron density in the constriction is less than its value in the wide region, then Eq (137) predicts a crossover at $B_{\text {erit }}$ to a strong-field regime of positive magnetoresistance described by

$$
R_{\mathrm{L}} \approx \frac{h}{2 e^{2}}\left(\frac{\hbar \omega_{\mathrm{c}}}{E_{\mathrm{F}}-E_{\mathrm{c}}}-\frac{\hbar \omega_{\mathrm{c}}}{E_{\mathrm{F}}}\right), \quad \text { if } B>B_{\mathrm{crt1}}
$$


The experimental results are well described by the solid curves following from Eq (137) (with $N_{\min }$ given by the square-well result (108), and with an added constant background resistance) The constriction in the present experiment is relatively long ( $L \approx 34 \mu \mathrm{m}$ ), and wide ( $W$ ranging from 02 to $10 \mu \mathrm{m})$ so that it does not exhibit quantized two-terminal conductance plateaux in the absence of a magnetic field For this reason the discreteness of $N_{\text {min }}$ was ignored in the theoretical curves in Fig 50 We emphasize, however, that $\mathrm{Eq}(137)$ is equally applicable to the quantized case, as observed by several groups ${ }^{307} 339-342$ (see Section 19)

The negative magnetoresistance (137) due to the suppression of the contact resistance is an additive contribution to the magnetoresistance of a long and narrow channel in the quasi-ballistic regime (if the voltage piobes are positioned on two wide 2DEG regions, connected by the channel) For a channel of length $L$ and a mean free path $l$ the zero-field contact resistance is a fraction $\sim l / L$ of the Drude resistance and may thus be ignored for $L \gg l$ The strong-field positive magnetoresistance (138) resulting from a different electron density in the channel may still be important, however The effect of the contact resistance may be suppressed to a large extent by using narrow voltage probes attached to the channel itself rather than to wide 2DEG regions As we will see in Section 16, such a solution no longer works in the ballistıc transport regime, because of the additional scattering induced ${ }^{289}$ by the voltage probes

\section{Coherent Electron Focusing}

A magnetic field may be used to focus the electrons injected by a point contact onto a second point contact Electron focusing in metals was originally conceived by Sharvin ${ }^{296}$ as a method to investigate the shape of the Fermı surface It has become a powerful tool in the study of surface scattering, ${ }^{343}$ and the electron-phonon interaction, ${ }^{344}$ as reviewed in Refs 305,345 , and 346 The experiment is the analogue in the solid state of magnetic focusing of electrons in vacuum Required is a large mean free path for the carriers at the Fermi surface, to ensure ballistic motion as in vacuum The mean free path should be much larger than the separation $L$ of the two

${ }^{339}$ S Washburn, A B Fowler, H Schmid, and D Kern, Phys Rev Lett 61, 2801 (1988)

${ }^{340} \mathrm{R}$ J Haug, A H MacDonald, P Streda, and K von Klitzing, Phys Rev Lett 61, 2797 (1988)

${ }^{341}$ R J Haug, J Kucera, P Streda, and K von Klitzing, Phys Rev B 39, 10892 (1989)

${ }^{342}$ B R Snell, P H Beton, P C Main, A Neves, J R Owers-Bradley, L Eaves, M Henını, O H

Hughes, S P Beaumont, and C D W Wilkinson, J Phys Condens Matter 1, 7499 (1989)

${ }^{343} \mathrm{~V}$ S Tsol, Pis'ma Zh Eksp Teor Fiz 19, 114 (1974) [JETP Lett 19, 70 (1974)], Zh Eksp

Teor Fiz 68, 1849 (1975) [Sov Phys JETP 41, 927 (1975)]

${ }^{344} \mathrm{P}$ C van Son, $\mathrm{H}$ van Kempen, and P Wyder, Phys Rev Lett 58, 1567 (1987)

${ }^{345}$ I K Yanson, Zh Eksp Teor Fiz 66, 1035 (1974) [Sov Phys JETP 39, 506 (1974)]

${ }^{346}$ A M Duif, A G M Jansen, and P Wyder, J Phys Condens Matter 1, 3157 (1989) 
point contacts Moreover, $L$ should be much larger than the point contact width $W$, to achieve optımal resolution In metals, electron focusing is essentially a classical phenomenon because the Fermi wavelength $\iota_{\Gamma} \sim 05 \mathrm{~nm}$ is much smaller than both $W \sim 1 \mu \mathrm{m}$ and $L \sim 100 \mu \mathrm{m}$ The ratios $\lambda_{\mathrm{F}} / L$ and $\lambda_{\mathrm{F}} / W$ are much larger in a $2 \mathrm{DEG}$ than in a metal, typically by factors of $10^{4}$ and $10^{2}$, respectively Coherent electron focusing ${ }^{59} 80347$ is possible in a 2DEG because of this relatively large value of the Fermi wavelength, and turns out to be strikingly different from classical electron focusing in metals

Electron focusing can be seen as a transmission experiment in electron optics (cf Ref 3 for a discussion from this point of view) An alternative point of view (emphasized in Refs 80 and 348) is that coherent electron focusing is a prototype of a nonlocal resistance measurement in the quantum ballistic transport regime, such as studied extensively in narrow-channel geometries ${ }^{310}$ Longitudinal resistances that are negative (not $\pm B$ symmetric) and dependent on the properties of the current and voltage contacts as well as on their separation, periodic and aperiodic magnetoresistance oscillations, absence of local equilibrium are all characteristic features of this transport regime that appear in a most extreme and bare form in the electron focusing geometry One reason for the simplification offered by this geometry is that the current and voltage contacts, being point contacts, are not nearly as invasive as the wide leads in a Hall bar geometry Another reason is that the electrons interact with only one boundary (nnstead of two in a narrow channel)

The outline of this section is as follows In Section 14 a the experimental results on coherent electron focusing ${ }^{5980}$ are presented A theoretical description ${ }^{80}{ }^{347}$ is given in Section $14 \mathrm{~b}$, in terms of mode interference in the waveguide formed by the magnetic field at the 2DEG boundary Apart from the intrinsic interest of electron focusing in a 2DEG, the experiment can also be seen as a method to study electron scattering, as in metals Two such applications ${ }^{108}{ }^{349}$ are discussed in Section $14 \mathrm{c}$ We restrict ourselves in this section to focusing by a magnetic field Electrostatic focusing ${ }^{350}$ is discussed in Section 15 b

\section{a Experiments}

The geometry of the experment ${ }^{59}$ in a $2 \mathrm{DEG}$ is the transverse focusing

${ }^{347} \mathrm{C}$ W J Beenakker, H van Houten, and B J van Wees, Europhys Lett 7, 359 (1988)

${ }^{348} \mathrm{C}$ W J Beenakker, H van Houten, and B J van Wees, Festkorperprobleme 29, 299 (1989)

${ }^{349} \mathrm{~J}$ Spector, H L Stormer, K W Baldwin, L N Pfelffer and K W West, Surf Scı 228, 283 (1990)

${ }^{350}$ U Sivan, M Heiblum, and C P Umbach, and H Shtrikman, Phys Rev B 41, 7937 (1990), J Spector, H L Stormer, K W Baldwin, L N Pfelffer and K W West, Appl Phys Lett 56, $1290(1990)$ 


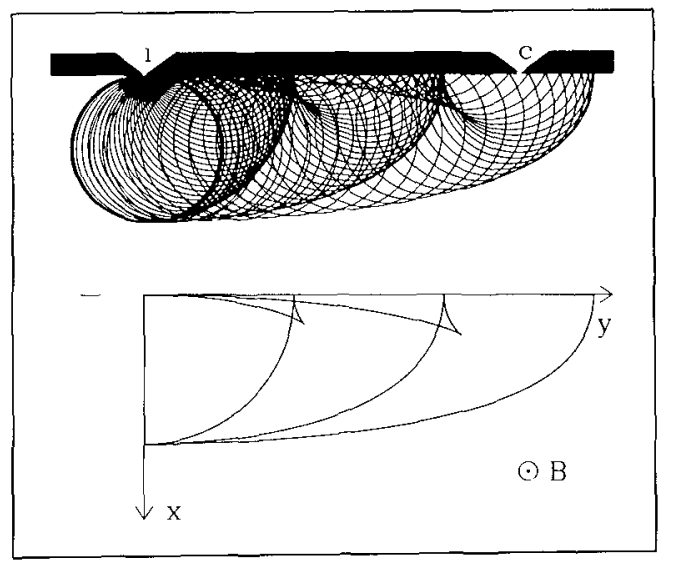

Fig 52. Illustration of classical electron focusing by a magnetic field. Top Skipping orbits along the 2DEG boundary The trajectories are drawn up to the third specular reflection. Bottom Plot of the caustics, which are the collection of focal points of the trajectories. Taken from $\mathrm{H}$ van Houten et al, Phys Rev B 39, 8556 (1989)

geometry of Tsoi ${ }^{343}$ and consists of two point contacts on the same boundary in a perpendicular magnetic field. (In metals one can also use the geometry of Sharvin ${ }^{296}$ with opposite point contacts in a longitudinal field. This is not possible in two dimensions.) Two point contacts and the intermediate 2DEG boundary are created electrostatically by means of the two split gates shown in Fig. 5b. Figure 52 illustrates electron focusing in two dimensions as it follows from the classical mechanics of electrons at the Fermi level. The injector (i) injects a divergent beam of electrons ballistically into the 2DEG. Electrons are detected if they reach the adjacent collector (c), after one or more specular reflections at the boundary connecting $i$ and $c$. (These are the skipping orbits discussed in Section 12.a.) The focusing action of the magnetic field is evident in Fig. 52 (top) from the black lines of high density of trajectories. These lines are known in optics as caustics and they are plotted separately in Fig. 52 (bottom). The caustics intersect the 2DEG boundary at multiples of the cyclotron diameter from the injector. As the magnetic field is increased, a series of these focal points shifts past the collector. The electron flux incident on the collector thus reaches a maximum whenever its separation $L$ from the injector is an integer multiple of $2 l_{\mathrm{cycl}}=2 \hbar k_{\mathrm{F}} / e B$. This occurs when $B=p B_{\text {focus }}, p=1,2, \ldots$, with

$$
B_{\text {focus }}=2 \hbar k_{\mathrm{F}} / e L .
$$

For a given injected current $I_{1}$ the voltage $V_{\mathrm{c}}$ on the collector is proportional to the incident flux. The classical picture thus predicts a series of equidistant peaks in the collector voltage as a function of magnetic field. 


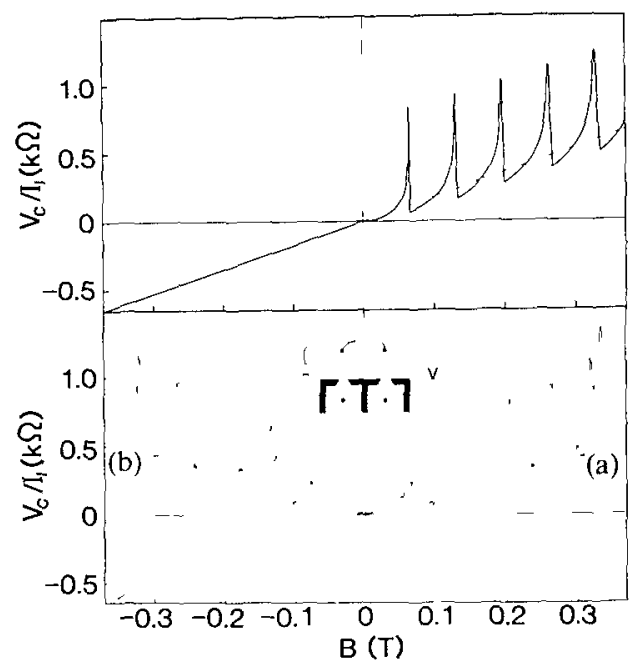

Fig. 53. Bottom: Experimental electron focusing spectrum ( $T=50 \mathrm{mK}, L=3.0 \mu \mathrm{m})$ in the generalized Hall resistance configuration depicted in the inset. The two traces $a$ and $b$ are measured with interchanged current and voltage leads, and demonstrate the injector-collector reciprocity as well as the reproducibility of the fine structure. Top: Calculated classical focusing spectrum corresponding to the experimental trace $a(50-\mathrm{nm}$-wide point contacts were assumed). The dashed line is the extrapolation of the classical Hall resistance seen in reverse fields. Taken from H. van Houten et al., Phys. Rev. B 39, 8556 (1989).

In Fig. 53 (top) we show such a classical focusing spectrum, calculated for parameters corresponding to the experiment discussed later $(L=3.0 \mu \mathrm{m}$, $k_{\mathrm{F}}=1.5 \times 10^{8} \mathrm{~m}^{-1}$ ). The spectrum consists of equidistant focusing peaks of approximately equal magnitude superimposed on the Hall resistance (dashed line). The $p$ th peak is due to electrons injected perpendicularly to the boundary that have made $p-1$ specular reflections between injector and collector. Such a classical focusing spectrum is commonly observed in metals, ${ }^{351,352}$ albeit with a decreasing height of subsequent peaks because of partially diffuse scattering at the metal surface. Note that the peaks occur in one field direction only. In reverse fields the focal points are at the wrong side of the injector for detection, and the normal Hall resistance is obtained. The experimental result for a 2DEG is shown in the bottom half of Fig. 53 (trace a; trace $b$ is discussed later). A series of five focusing peaks is evident at the expected positions. The observation of multiple focusing peaks immediately

${ }^{351}$ P. A. M. Benistant, Ph.D. thesis, University of Nijmegen, The Netherlands, 1984; P. A. M. Benistant, A. P. van Gelder, H. van Kempen, and P. Wyder, Phys. Rev. B 32, 3351 (1985). ${ }^{352}$ P. A. M. Benistant, G. F. A. van de Walle, H. van Kempen, and P. Wyder, Phys. Rev. B 33, 690 (1986). 
implies that the electrostatically defined 2DEG boundary scatters predominantly specularly. (This finding ${ }^{59}$ is supported by the magnetoresistance experiments of Thornton et al. ${ }^{107}$ in a narrow split-gate channel; cf. Section 5.) Figure 53 is obtained in a measuring configuration (inset) in which an imaginary line connecting the voltage probes crosses that between the current source and drain. This is the configuration for a generalized Hall resistance measurement. If the crossing is avoided, one measures a longitudinal resistance, which shows the focusing peaks without a superimposed Hall slope. This longitudinal resistance periodically becomes negative. This is a classical result ${ }^{80}$ of magnetic defocusing, which causes the probability density near the point contact voltage probe to be reduced with respect to the spatially averaged probability density that determines the voltage on the wide voltage probe (cf. the regions of reduced density between lines of focus in Fig. 52).

On the experimental focusing peaks a fine structure is resolved at low temperatures (below $1 \mathrm{~K}$ ). The fine structure is well reproducible but sample-dependent. A nice demonstration of the reproducibility of the fine structure is obtained upon interchanging current and voltage leads, so that the injector becomes the collector, and vice versa. The resulting focusing spectrum shown in Fig. 53 (trace b) is almost the precise mirror image of the original one (trace a), although this particular device had a strong asymmetry in the widths of injector and collector. The symmetry in the focusing spectra is an example of the general reciprocity relation (12.16). If one applies the Büttiker equations (12.12) to the electron focusing geometry (as is done in Section 19), one finds that the ratio of collector voltage $V_{\mathrm{c}}$ to injector current $I_{\mathrm{i}}$ is given by

$$
\frac{V_{\mathrm{c}}}{I_{\mathrm{i}}}=\frac{2 e^{2}}{h} \frac{T_{\mathrm{i} \rightarrow \mathrm{c}}}{G_{\mathrm{i}} G_{\mathrm{c}}},
$$

where $T_{\mathrm{i} \rightarrow \mathrm{c}}$ is the transmission probability from injector to collector, and $G_{\mathrm{i}}$ and $G_{\mathrm{c}}$ are the conductances of the injector and collector point contact. Since $T_{\mathrm{i} \rightarrow \mathrm{c}}(B)=T_{\mathrm{c} \rightarrow \mathrm{i}}(-B)$ and $\mathrm{G}(\mathrm{B})=\mathrm{G}(-\mathrm{B})$, this expression for the focusing spectrum is manifestly symmetric under interchange of injector and collector with reversal of the magnetic field.

The fine structure on the focusing peaks in Fig. 53 is the first indication that electron focusing in a $2 \mathrm{DEG}$ is qualitatively different from the corresponding experiment in metals. At higher magnetic fields the resemblance to the classical focusing spectrum is lost; see Fig. 54. A Fourier transform of the spectrum for $B \geqslant 0.8 \mathrm{~T}$ (inset in Fig. 54) shows that the large-amplitude highfield oscillations have a dominant periodicity of $0.1 \mathrm{~T}$, which is approximately the same as the periodicity $B_{\text {focus }}$ of the much smaller focusing peaks at low magnetic fields $\left(B_{\text {focus }}\right.$ in Fig. 54 differs from Fig. 53 because of a smaller 


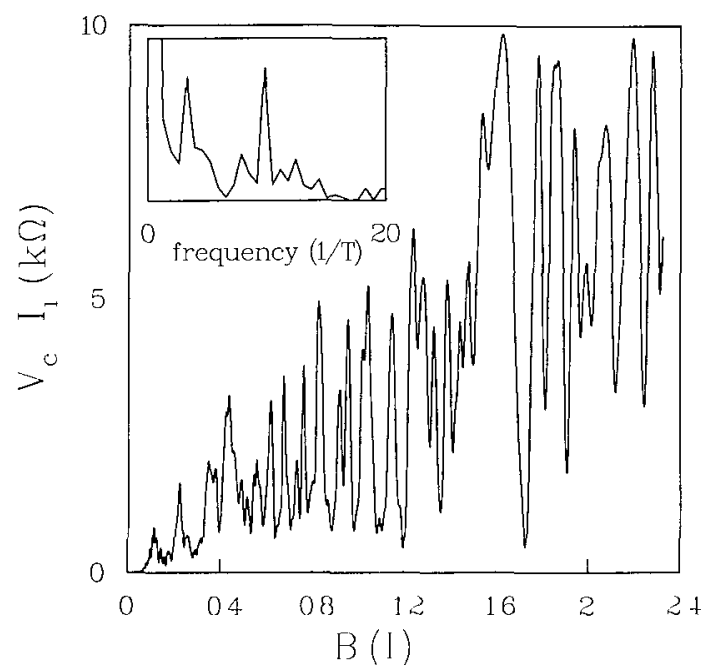

Fig 54 Experimental electron focusing spectrum over a larger field range and for very narrow point contacts (estımated width $20-40 \mathrm{~nm}, T=50 \mathrm{mK}, L=15 \mu \mathrm{m}$ ) The inset gives the Fourier transform for $B \geqslant 08 T$ The high-field oscillations have the same dominant periodicity as the low-field focusing peaks, but with a much larger amplitude Taken from $\mathrm{H}$ van Houten $e t$ al, Phys Rev B 39, 8556 (1989)

$L=15 \mu \mathrm{m})$ This dominant periodicity can be explained in terms of quantum interference between the different skipping orbits from injector to collector or in terms of interference of coherently excited edge channels, as we discuss in the following subsection The experimental implication is that the injector acts as a coherent point source with the coherence maintained over a distance of several microns to the collector

\section{b Theory}

To explain the characteristic features of the coherent electron focusing experiments we have described, we must go beyond the classical description ${ }^{80347}$ As discussed in Section 12, quantum ballistıc transport along the 2DEG boundary in a magnetıc field takes place via magnetıc edge states, which form the propagatıng modes at the Fermı level Since the injector has a width below $\lambda_{\mathrm{F}}$, it excites these modes coherently For $k_{\mathrm{F}} L \gg 1$ the interference of modes at the collector is dominated by therr rapidly varying phase factors $\exp \left(\imath k_{n} L\right)$ The wave number $k_{n}$ corresponds classically to the separation of the center of the cyclotron orbit from the 2DEG boundary [Eq (12 5)] In the Landau gauge $\mathbf{A}=(0, B x, 0)$ (with the axis chosen as in Fig 52) one has $k_{n}=k_{\mathrm{F}} \sin \alpha_{n}$, where $\alpha$ is the angle with the $x$-axis under which the cyclotron orbit is reflected from the boundary The quantized values $\alpha_{n}$ follow 


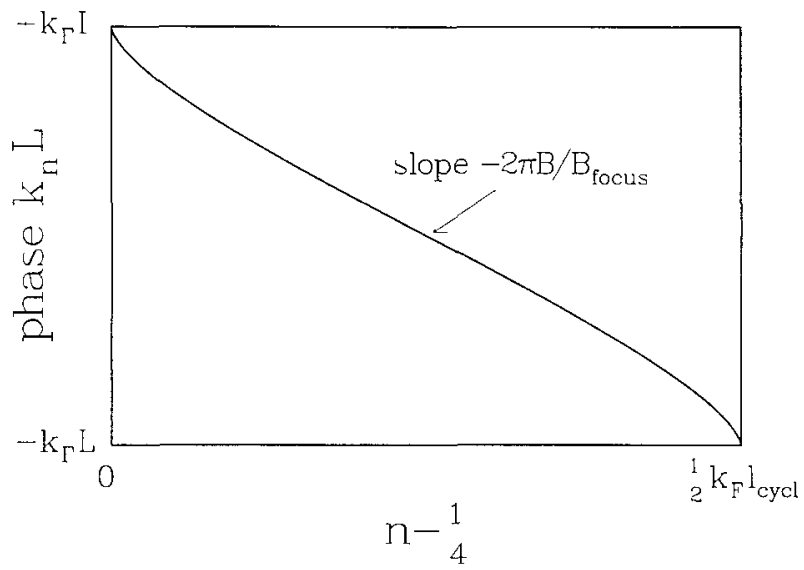

FIG 55 Phase $k_{n} L$ of the edge channels at the collector calculated from Eq (143) Note the domain of approximately linear $n$-dependence of the phase, responsible for the oscillations with $B_{\text {focus }}$-periodicity Taken from $\mathrm{H}$ van Houten et al, Phys Rev B 39, 8556 (1989)

in this semiclassical description from the Bohr-Sommerfeld quantization rule (126) that the flux enclosed by the cyclotron orbit and the boundary equals $\left(n-\frac{1}{4}\right) h / e$ [the phase shift $\gamma$ in Eq (126) equals $\pi / 2$ for an edge state at an infinite barrier potential] Simple geometry shows that this requires that

$$
\frac{\pi}{2}-\alpha_{n}-\frac{1}{2} \sin 2 \alpha_{n}=\frac{2 \pi}{k_{\mathrm{F}} l_{\mathrm{cycl}}}\left(n-\frac{1}{4}\right), \quad n=1,2, \quad, N
$$

As plotted in Fig 55, the dependence on $n$ of the phase $k_{n} L$ is close to linear in a broad interval This also follows from expansion of Eq (143) around $\alpha_{n}=0$, which gives

$$
k_{n} L=\text { constant }-2 \pi n \frac{B}{B_{\text {focus }}}+k_{\mathrm{F}} L \times \text { order }\left(\frac{N-2 n}{N}\right)^{3}
$$

If $B / B_{\text {focus }}$ is an integer, a fraction of order $\left(1 / k_{\mathrm{F}} L\right)^{1 / 3}$ of the $N$ edge states interfere constructively at the collector Because of the $1 / 3$ power, this is a substantial fraction even for the large $k_{\mathrm{F}} L \sim 10^{2}$ of the experiment The resulting mode interference oscillations with $B_{\text {focus }}$-periodicity can become much larger than the classical focusing peaks This has been shown in Refs 347 and 80 , where the transmission probability $T_{1 \rightarrow c}$ was calculated in the WKB approximation with neglect of the finite width of the injector and detector From Eq (142) the focusing spectrum is then obtained in the form

$$
\frac{V_{\mathrm{c}}}{I_{1}}=\frac{h}{2 e^{2}}\left|\frac{1}{N} \sum_{n=1}^{N} \mathrm{e}^{\imath k_{n} L}\right|^{2}
$$




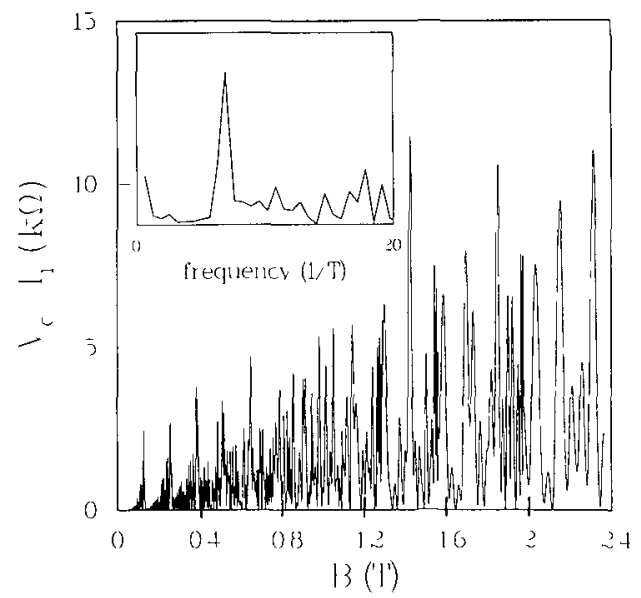

Fig. 56. Focusing spectrum calculated from Eq. (14.5), for parameters corresponding to the experimental Fig. 54. The inset shows the Fourier transform for $B \geqslant 0.8 T$. Infinitesimally small point contact widths are assumed in the calculation. Taken from C. W. J. Beenakker et al,, Festkörperprobleme 29, 299 (1989).

which is plotted in Fig. 56 for parameter values corresponding to the experimental Fig. 54. The inset shows the Fourier transform for $B \geqslant 0.8 \mathrm{~T}$.

There is no detailed one-to-one correspondence between the experimental and theoretical spectra. No such correspondence was to be expected in view of the sensitivity of the experimental spectrum to small variations in the voltage on the gate defining the point contacts and the 2DEG boundary. Those features of the experimental spectrum that are insensitive to the precise measurement conditions are, however, well reproduced by the calculation: We recognize in Fig. 56 the low-field focusing peaks and the large-amplitude high-field oscillations with the same $B_{\text {focus }}$-periodicity. The high-field oscillations range from about 0 to $10 \mathrm{k} \Omega$ in both theory and experiment. The maximum amplitude is not far below the theoretical upper bound of $h / 2 e^{2} \approx 13 \mathrm{k} \Omega$, which follows from Eq. (14.5) if we assume that all the modes interfere constructively. This indicates that a maximal phase coherence is realized in the experiment and implies that the experimental injector and collector point contacts resemble the idealized point source/detector in the calculation.

\section{c. Scattering and Electron Focusing}

Scattering events other than specular boundary scattering can be largely ignored for the relatively small point contact separations $L \leqslant 3 \mu \mathrm{m}$ in the experiments discussed earlier ${ }^{59,80}$ (any other inelastic or elastic scattering 


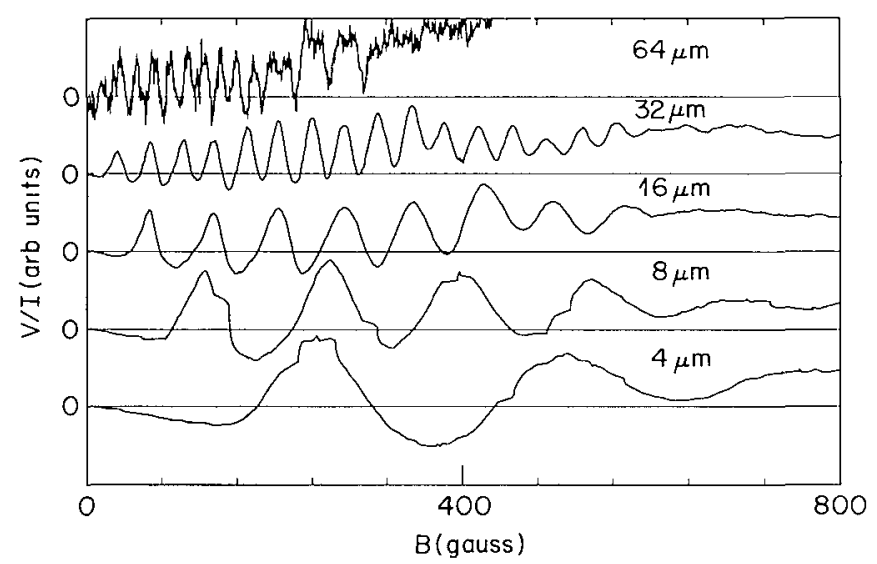

FIG 57 Experimental electron focusing spectra (in the generalized longitudinal resistance configuration) at $03 \mathrm{~K}$ for five different injector-collector separations in a very high mobility material The vertical scale varies among the curves Taken from J Spector et al Surf Scı 228, 283 (1990)

events would have been detected as a reduction of the oscillations with $B_{\text {focus }}$ periodicity below the theoretical estimate) Spector et al ${ }^{349}$ have repeated the experiments for larger $L$ to study scattering processes in an ultrahigh mobility $3533542 \mathrm{DEG}\left(\mu_{\mathrm{e}}=55 \times 10^{6} \mathrm{~cm}^{2} / \mathrm{V} \mathrm{s}\right)$ They used relatively wide point contacts (about $1 \mu \mathrm{m}$ ) so that electron focusing was in the classical regime In Fig 57 we reproduce their experimental results for point contact separations up to $64 \mu \mathrm{m}$ The peaks in the focusing spectrum for a given $L$ have a roughly constant amplitude, indicating that scattering at the boundary is mostly specular rather than diffusive-in agreement with the experiments of Ref 59 Spector et al $^{349}$ find that the amplitude of the focusing peaks decreases exponentially with increasing $L$, due to scattering in the electron gas (see Fig 58) The decay $\exp \left(-L / L_{0}\right)$ with $L_{0} \approx 10 \mu \mathrm{m}$ implies an effective mean free path (measured along the arc of the skipping orbits) of $L_{0} \pi / 2 \approx 15 \mu \mathrm{m}$ This is smaller than the transport mean free path derived from the conductivity by about a factor of 2 , which may point to a greater sensitivity of electron focusing to forward scattering

Electron focusing by a magnetic field may also play a role in geometries other than the double-point contact geometry of Fig 52 One example is mentioned in the context of junction scattering in a cross geometry in Section 16 Another example is the experiment by Nakamura et al ${ }^{108}$ on the magnetoresistance of equally spaced narrow channels in parallel (see Fig 59)

${ }^{353}$ L Pfeiffer, K W West, H L Stormer, and K W Baldwin, Appl Phys Lett 55, 1888 (1989)

${ }^{354} \mathrm{C}$ T Foxon, J J Harris D Hilton, J Hewett, and C Roberts, Semicond Sct Technol 4, 582 (1989), C T Foxon and J J Harris, Philips $J$ Res 41, 313 (1986) 


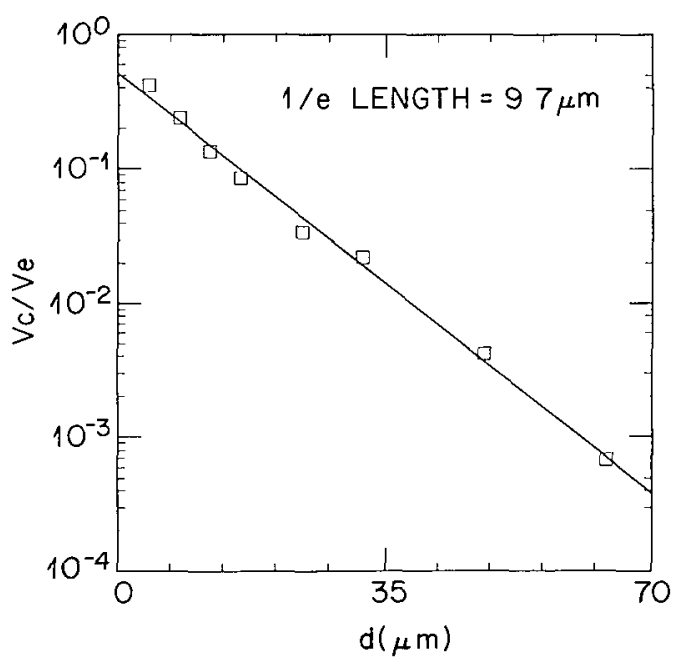

FIG 58 Exponential decay of the oscillation amplitude of the collector voltage (normalized by the injector voltage) as a function of injector-collector separation $d$ (denoted by $L$ in the text) Taken from J Spector et al, Surf Scl 228, 283 (1990)

Resistance peaks occur in this experiment when electrons that are transmitted through one of the channels are focused back through another channel The resistance peaks occur at $B=(n / m) B_{\text {focus }}$, where $B_{\text {focus }}$ is given by Eq (141) with $L$ the spacing of adjacent channels The identification of the various peaks in Fig 59 is given in the inset Nakamura et al. ${ }^{108}$ conclude from the rapidly diminishing height of consecutive focusing peaks (which require an increasing number of specular reflections) that there is a large probability of diffuse boundary scattering The reason for the difference with the experiments discussed previously is that the boundary in the experiment of Fig 59 is defined by focused ion beam lithography, rather than electrostatically by means of a gate As discussed in Section 5, the former technique may introduce a considerable boundary roughness

Electron focusing has been used by Willamson et al ${ }^{355}$ to study scattering processes for "hot" electrons, with an energy in excess of the Ferm1 energy, and for "cool" holes, or empty states in the conduction band below the Fermı level (see Ref 307 for a review) An interesting aspect of hot-electron focusing is that it allows a measurement of the local electrostatic potential drop across a current-carrying quantum point contact, ${ }^{355}$ something that is not possible using conventional resistance measurements, where the sum of electrostatic 


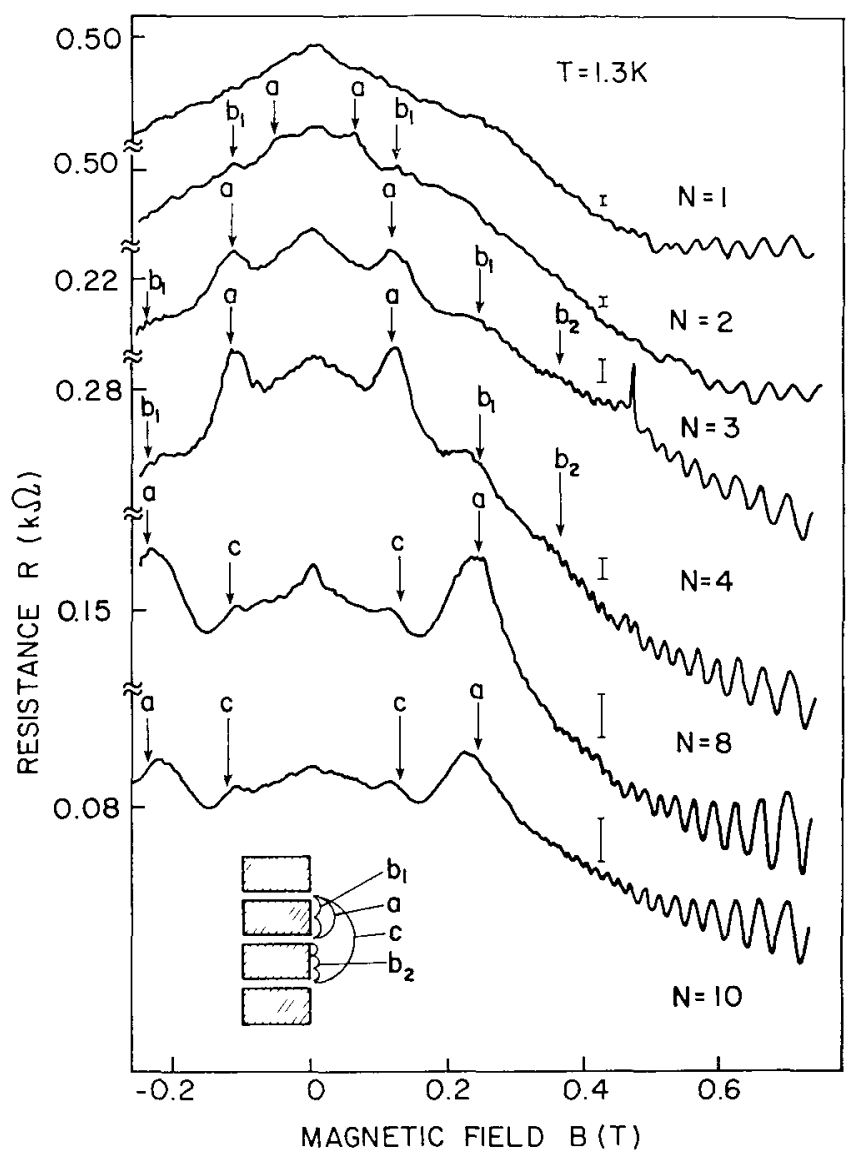

FIG. 59. Magnetoresistance of $N$ constrictions in parallel at $1.3 \mathrm{~K}$. The arrows indicate the oscillations due to electron focusing, according to the mechanisms illustrated in the inset. The resistance scale is indicated by $10 \Omega$ bars. Taken from K. Nakamura et al., Appl. Phys. Lett. 56, 385 (1990).

and chemical potentials is measured. The importance of such alternative techniques to study electrical conduction has been stressed by Landauer. ${ }^{356}$

\section{Collimation}

The subject of this section is the collimation of electrons injected by a point contact ${ }^{329}$ and its effect on transport measurements in geometries

${ }^{356} \mathrm{R}$. Landauer, in "Analogies in Optics and Microelectronics" (W. van Haeringen and D.

Lenstra, eds.). Kluwer Academic, Dordrecht, 1990. 
involving two opposite point contacts ${ }^{327} 357$ Collimation (1 e, the narrowing of the angular injection distributions) follows from the constraints on the electron momentum imposed by the potential barrier in the point contact (barrier colltmation), and by the gradual widening of the point contact at its entrance and exit (horn collumation) We summarize the theory in Section 15 a The effect was originally proposed ${ }^{329}$ to explain the remarkable observation of Wharam et $a l^{357}$ that the series resistance of two opposite point contacts is considerably less than the sum of the two individual resistances (Section $15 \mathrm{c}$ ) A direct experimental proof of collımation was provided by Molenkamp et $a l,{ }^{327}$ who measured the deflection of the injected beam of electrons in a magnetic field (Section 15 b) A related experiment by Sivan et al ${ }^{350}$ aimed at the demonstration of the focusing action of an electrostatic lens, is also discussed in this subsection The collimation effect has an importance in ballistic transport that goes beyond the point contact geometry It will be shown in Section 16 that the phenomenon is at the origin of a variety of magnetoresistance anomalies in narrow multiprobe conductors ${ }^{358-360}$

\section{a Theory}

Since collimation follows from classical mechanics, a semiclassical theory is sufficient to describe the essential phenomena, as we now discuss (following Refs 329 and 311) Semiclassically, collimation results from the adiabatic invariance of the product of channel width $W$ and absolute value of the transverse momentum $\hbar k_{y}$ (this product is proportional to the action for motion transverse to the channel) ${ }^{361}$ Therefore, if the electrostatic potential in the point contact region is sufficiently smooth, the quantity $S=\left|k_{y}\right| W$ is approximately constant from point contact entrance to exit Note that $S / \pi$ corresponds to the quantum mechanical 1D subband index $n$ The quantum mechanical criterion for adiabatic transport was derived by Yacoby and Imry $^{326}$ (see Section 13) As was discussed there, adiabatic transport breaks down at the exit of the point contact, where it widens abruptly into a $2 \mathrm{DEG}$ of essentially infinite width Collimation reduces the injection/acceptance cone of the point contact from its original value of $\pi$ to a value of $2 \alpha_{\operatorname{mzx}}$ This effect 1s illustrated in Fig 60 Electrons incident at an angle $|\alpha|>\alpha_{\max }$ from normal incidence are reflected (The geometry of Fig 60b is known in optics as a conical reflector ${ }^{362}$ ) Vice versa, all electrons leave the constriction at an angle

\footnotetext{
${ }^{357}$ D A Wharam, M Pepper, H Ahmed, J E F Frost, D G Hasko, D C Peacock, D A Ritchie, and G A C Jones, $J$ Phys C 21, L887 (1988)

${ }^{358} \mathrm{H}$ U Baranger and A D Stone, Phys Rev Lett 63, 414 (1989), also in Ref 16

${ }^{359} \mathrm{C}$ W J Beenakker and $\mathrm{H}$ van Houten, Phys Rev Lett 63, 1857 (1989)

${ }^{360} \mathrm{C}$ W J Beenakker and $\mathbf{H}$ van Houten, in Ref 17

${ }^{361}$ L D Landau and E M Lifshitz, "Mechanics" Pergamon, Oxford, 1976

${ }^{362}$ N S Kapany, in "Concepts of Classical Optics" (J Strong, ed ) Freeman, San Francisco, 1958
} 

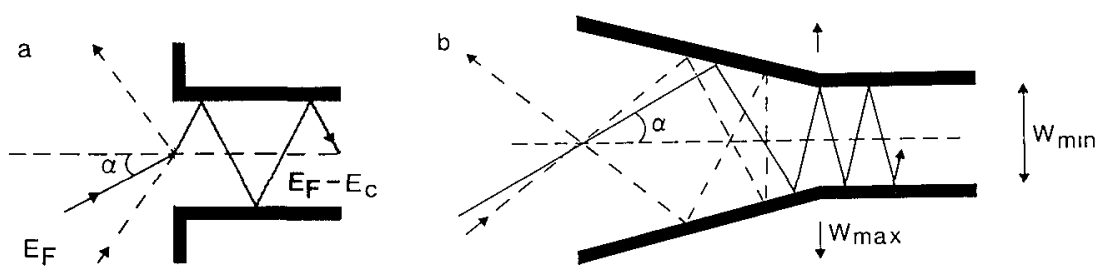

Fig 60 Illustration of the collimation effect for an abrupt constriction (a) containing a potential barrier of height $E_{\mathrm{c}}$ and for a horn-shaped constriction (b) that is flared from a width $W_{\min }$ to $W_{\max }$ The dash-dotted trajectories approaching at an angle $\alpha$ outside the injectiondcceptance cone are reflected Taken from $\mathrm{H}$ van Houten and $\mathrm{C} W \mathrm{~J}$ Beenakker, in Nanostructure Physics and Fabrication' (M Reed and W P Kırk, eds) Academic, New York, 1989

$|\alpha|<\alpha_{\operatorname{mix}}$ (1 e, the injected electrons form a collımated beam of angular opening $2 \alpha_{\mathrm{m} \text { x }}$ )

To obtain an analytic expression for the collimation effect, we describe the shape of the potential in the point contact region by three parameters $W_{\min }$, $W_{\mathrm{m} \text { ix }}$, and $E_{\mathrm{c}}$ (see Fig 60) We consider the case that the point contact has its minımal width $W_{\mathrm{min}}$ at the point where the barrier has its maximal height $E_{\mathrm{c}}$ above the bottom of the conduction band in the broad regions At that point the largest possible value of $S$ is

$$
S_{1} \equiv\left(2 m / \hbar^{2}\right)^{1 / 2}\left(E_{\mathrm{F}}-E_{\mathrm{c}}\right)^{1 / 2} W_{\mathrm{min}}
$$

We assume that adiabatic transport (1 e, $S=$ constant) holds up to a point of zero barrier herght and maximal width $W_{\mathrm{mux}}$ The abrupt separation of adiabatic and nonadiabatic regions is a simplification that can be, and has been, tested by numerical calculations (see below) At the point contact exit, the largest possible value of $S$ is

$$
S_{2} \equiv\left(2 m / \hbar^{2}\right)^{1 / 2}\left(E_{\mathrm{F}}\right)^{1 / 2} \sin \alpha_{\max } W_{\operatorname{mix}}
$$

The invarrance of $S$ implies that $S_{1}=S_{2}$, hence,

$$
\alpha_{\max }=\arcsin \left(\frac{1}{f}\right), \quad f \equiv\left(\frac{E_{\mathrm{F}}}{E_{\mathrm{F}}-E_{\mathrm{c}}}\right)^{1 / 2} \frac{W_{\max }}{W_{\min }}
$$

The collimation factor $f \geqslant 1$ is the product of a term describing the collimating effect of a barrier of height $E_{\mathrm{c}}$ (barrier collimation) and a term describing collimation due to a gradual widening of the point contact width from $W_{\min }$ to $W_{\mathrm{m} \text { x }}$ (horn collımation) In the adiabatic approximation, the angular injection distribution $P(\alpha)$ is proportional to $\cos \alpha$ with an abrupt truncation at $\pm \alpha_{\max }$ The cosine angular dependence follows from the cosine distribution of the incident flux in combination with time-reversal symmetry and is thus not affected by the reduction of the injection-acceptance cone 
We therefore conclude that in the adiabatic approximation $P(\alpha)$ (normalized to unity) is given by

$$
\begin{aligned}
P(\alpha) & =\frac{1}{2} f \cos \alpha, & & \text { if }|\alpha|<\arcsin (1 / f), \\
& =0, & & \text { otherwise }
\end{aligned}
$$

We defer to Section 15 b a comparison of the analytical result (15 2) with a numerical calculation

Barrier collımation does not require adiabaticity For an abrupt barrier, collimation simply results from transverse momentum conservation, as in Fig 60a, leading directly to Eq (152) (The total external reflection at an abrupt barrier for trajectories outside the collimation cone is similar to the optical effect of total internal reflection at a boundary separating a region of high refractive index from a region of small refractive index, see the end of Section $15 \mathrm{~b}$ ) A related collimation effect resulting from transverse momentum conservation occurs if electrons tunnel through a potential barrier Since the tunneling probability through a high potential barrier is only weakly dependent on energy, it follows that the strongest collimation is to be expected if the barrer height equals the Fermi energy On lowering the barrier below $E_{\mathrm{F}}$ ballistic transport over the barrier dominates, and the collimation cone widens according to Eq (152) A quantum mechanical calculation of barrier collimation may be found in Ref 363

The injection distribution (152) can be used to obtain (in the semiclassical limit) the direct transmission probability $T_{\mathrm{d}}$ between two opposite identical point contacts separated by a large distance $L$ To this end, first note that $T_{\mathrm{d}} / N$ is the fraction of the injected current that reaches the opposite point contact (since the transmission probability through the first point contact is $N$, for $N$ occupied subbands in the point contact) Electrons injected within a cone of opening angle $W_{\max } / L$ centered at $\alpha=0$ reach the opposite point contact and are transmitted If this opening angle is much smaller than the total opening angle $2 \alpha_{\mathrm{m}_{1 x}}$ of the beam, then the distribution function $P(\alpha)$ can be approximated by $P(0)$ within this cone This approximation requires $W_{\max } / L \ll 1 / f$, which is satisfied experimentally in devices with a sufficiently large point contact separation We thus obtain $T_{\mathrm{d}} / N=P(0) W_{\max } / L$, which, using Eq (152), can be written as ${ }^{329}$

$$
T_{\mathrm{d}}=f\left(W_{\max } / 2 L\right) N
$$

This simple analytical formula can be used to describe the experiments on transport through identical opposite point contacts in terms of one empirical parameter $f$, as discussed in the following subsections

${ }^{363} \mathrm{H}$ de Raedt, N Garcia, and J J Saenz, Phys Rev Lett 63, 2260 (1989), N Garcia, J J Saenz, and H de Raedt, J Phys Condens Matter 1, 9931 (1989) 


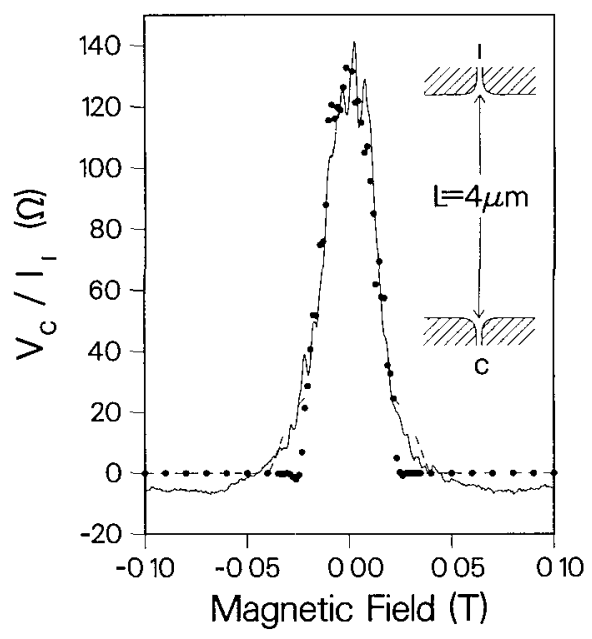

FIG 61 Detection of a collimated electron beam over a distance of $4 \mu \mathrm{m}$. In this four-terminal measurement, two ohmic contacts to the 2DEG region between the point contacts are used: One of these acts as a drain for the current $I_{1}$ through the injector, and the other is used as a zeroreference for the voltage $V_{c}$ on the collector. The drawn curve is the experimental data at $T=18 \mathrm{~K}$ The black dots are the result of a semiclassical simulation, using a hard-wall potential with contours as shown in the inset The dashed curve results from a simulation without collımation (corresponding to rectangular corners in the potential contour). Taken from L. W Molenkamp et al, Phys Rev. B 41, 1274 (1990)

\section{b. Magnetic Deflection of a Collimated Electron Beam}

A method ${ }^{31,329}$ to sensitively detect the collimated electron beam injected by a point contact is to sweep the beam past a second opposite point contact by means of a magnetic field. The geometry is shown in Fig. 61 (inset). The current $I_{1}$ through the injecting point contact is drained to ground at one or two (the difference is not essential) ends of the 2DEG channel separating the point contacts. The opposite point contact, the collector, serves as a voltage probe (with the voltage $V_{\mathrm{c}}$ being measured relative to ground). In the case that both ends of the 2DEG channel are grounded, the collector voltage divided by the injected current is given by

$$
\frac{V_{\mathrm{c}}}{I_{\mathrm{t}}}=\frac{1}{G} \frac{T_{\mathrm{d}}}{N}, \quad T_{\mathrm{d}} \ll N,
$$

with $G=\left(2 e^{2} / h\right) N$ the two-terminal conductance of the individual point contact (both point contacts are assumed to be identical) and $T_{\mathrm{d}}$ the direct transmission probability between the two point contacts calculated in Section 15.4. Equation (15.4) can be obtained from the Landauer-Büttiker 
formalism (as done in Ref. 311) or simply by noting that the current $I_{\mathrm{i}} T_{\mathrm{d}} / N$ incident on the collector has to be counterbalanced by an equal outgoing current $G V_{\mathrm{c}}$. In the absence of a magnetic field, we obtain [using Equation (15.3) for the direct transmission probability]

$$
\frac{V_{\mathrm{c}}}{I_{\mathrm{i}}}=\frac{h}{2 e^{2}} f^{2} \frac{\pi}{2 k_{\mathrm{F}} L},
$$

where $k_{\mathrm{F}}$ is the Fermi wave vector in the region between the point contacts. In an experimental situation $L$ and $k_{\mathrm{F}}$ are known, so the collimation factor $f$ can be directly determined from the collector voltage by means of Eq. (15.5).

The result (15.5) holds in the absence of a magnetic field. A small magnetic field $B$ will deflect the collimated electron beam past the collector. Simple geometry leads to the criterion $L / 2 l_{\text {cycl }}=\alpha_{\max }$ for the cyclotron radius at which $T_{\mathrm{d}}$ is reduced to zero by the Lorentz force (assuming that $L \gg W_{\max }$ ). One would thus expect to see in $V_{\mathrm{c}} / I_{\mathrm{i}}$ a peak around zero field, of height given by Eq. (15.5) and of width

$$
\Delta B=\left(4 \hbar k_{\mathrm{F}} / e L\right) \arcsin (1 / f),
$$

according to Eq. (15.1).

In Fig. 61 this collimation peak is shown (solid curve), as measured by Molenkamp et al. ${ }^{327}$ at $T=1.2 \mathrm{~K}$ in a device with a $L=4.0-\mu \mathrm{m}$ separation between injector and collector. In this measurement only one end of the region between the point contacts was grounded-a measurement configuration referred to in narrow Hall bar geometries as a bend resistance measurement ${ }^{289,364}$ (cf. Section 16). One can show, using the LandauerBüttiker formalism, ${ }^{5}$ that the height of the collimation peak is still given by Eq. (15.5) if one replaces ${ }^{327} f^{2}$ by $f^{2}-\frac{1}{2}$. The expression (15.6) for the width is not modified. The experimental result in Fig. 61 shows a peak height of $\approx 150 \Omega$ (measured relative to the background resistance at large magnetic fields). Using $L=4.0 \mu \mathrm{m}$ and the value $k_{\mathrm{F}}=1.1 \times 10^{8} \mathrm{~m}^{-1}$ obtained from Hall resistance measurements in the channel between the point contacts, one deduces a collimation factor $f \approx 1.85$. The corresponding opening angle of the injection/acceptance cone is $2 \alpha_{\max } \approx 65^{\circ}$. The calculated value of $f$ would imply a width $\Delta B \approx 0.04 \mathrm{~T}$, which is not far from the measured full width at half maximum of $\approx 0.03 \mathrm{~T}$.

The experimental data in Fig. 61 are compared with the result ${ }^{327}$ from a numerical simulation of classical trajectories of the electrons at the Fermi level (following the method of Ref. 329). This semiclassical calculation was performed in order to relax the assumption of adiabatic transport in the point

${ }^{364}$ Y. Takagaki, K. Gamo, S. Namba, S. Ishida, S. Takaoka, K. Murase, K. Ishibashi, and Y. Aoyagi, Solid State Comm. 68, 1051 (1988); 71, 809 (1989). 


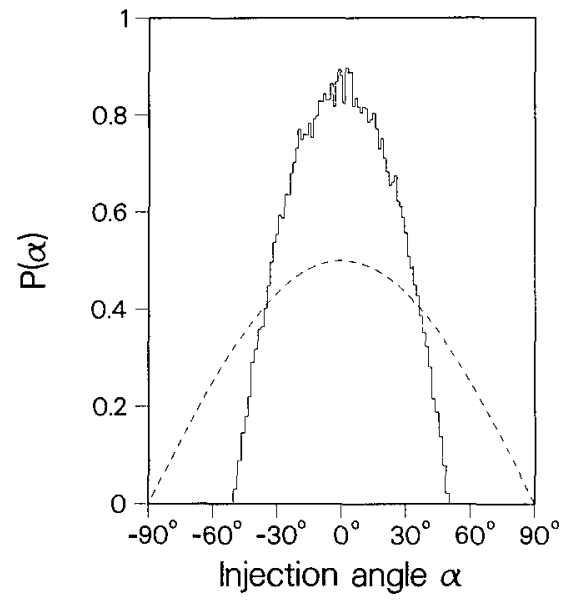

FIG. 62. Calculated angular injection distributions in zero magnetic field. The solid histogram is the result of a simulation of the classical trajectories at the Fermi energy in the geometry shown in the inset of Fig. 61. The dotted curve follows from the adiabatic approximation (15.2), with the experimental collimation factor $f=1.85$. The dashed curve is the cosine distribution in the absence of any collimation. Taken from L. W. Molenkamp et al., Phys. Rev. B. 41, 1274 (1990).

contact region, and of small $T_{\mathrm{d}} / N$, on which Eqs. (15.3) and (15.5) are based. The dashed curve is for point contacts defined by hardwall contours with straight corners (no collimation); the dots are for the smooth hardwall contours shown in the inset, which lead to collimation via the horn effect (cf. Fig. 60b; the barrier collimation of Fig. 60a is presumably unimportant at the small gate voltage used in the experiment and is not taken into account in the numerical simulation). The angular injection distributions $P(\alpha)$ that follow from these numerical simulations are compared in Fig. 62 (solid histogram) with the result (15.2) from the adiabatic approximation for $f=1.85$ (dotted curve). The uncollimated distribution $P(\alpha)=(\cos \alpha) / 2$ is also shown for comparison (dashed curve). Taken together, Figs. 61 and 62 unequivocally demonstrate the importance of collimation for the transport properties, as well as the adequateness of the adiabatic approximation as an estimator of the collimation cone.

Once the point contact width becomes less than a wavelength, diffraction inhibits collimation of the electron beam. In the limit $k_{\mathrm{F}} W \ll 1$, the injection distribution becomes proportional to $\cos ^{2} \alpha$ for all $\alpha$, independent of the shape of the potential in the point contact region. ${ }^{80,313}$ The coherent electron focusing experiments ${ }^{59,80}$ discussed in Sections 14.a and 14.b were performed in this limit.

We conclude this subsection by briefly discussing an alternative way to increase the transmission probability between two opposite point contacts, which is focusing of the injected electron beam onto the collector. Magnetic focusing, discussed in Section 14 for adjacent point contacts, cannot be used for opposite point contacts in two dimensions (unlike in three dimensions, where a magnetic field along the line connecting the point contacts will focus 


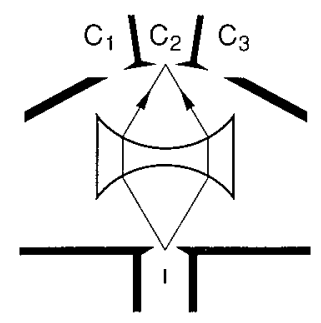

FIG 63 Electrostatic focusing onto a collector (c2) of an injected electron beam (at 1 ) by means of a concave lens corresponding to a region of reduced electron density Focusing in such an arrangement was detected expermentally ${ }^{350}$

the beam ${ }^{296}$ ) A succesful demonstration of electrostatic focusing was recently reported by Sivan et al and by Spector et al ${ }^{350}$ The focusing is acheved by means of a potential barrier of a concave shape, created as a region of reduced density in the 2DEG by means of a gate between the injector and the collector (see F1g 63) A focusing lens for electrons is concave because electrons approaching a potential barrier are deflected in a direction perpendicular to the normal This is an amusing difference with light, which is deflected toward the normal on entering a more dense medium, so an optical focusing lens is convex The different dispersion laws are the origin of this different behavior of light and electrons ${ }^{350}$

\section{c Series Resistance}

The first experimental study of ballistic transport through two opposite point contacts was carried out by Wharam et al, ${ }^{357}$ who discovered that the series resistance is considerably less than the sum of the two individual resistances Sugsequent experiments confirmed this result ${ }^{365366}$ The theoretical explanation ${ }^{329}$ of this observation is that collimation of the electrons injected by a point contact enhances the direct transmission probability through the opposite point contact, thereby significantly reducing the series resistance below its ohmic value We will discuss the transport and magnetotransport in this geometry We will not consider the alternative geometry of two adjacent point contacts in parallel (studied in Refs 367369) In that geometry the collimation effect cannot enhance the coupling of the two point contacts, so only small deviations from Ohm's law are to be expected

\footnotetext{
${ }^{365}$ Y Hirayama and T Saku, Solld State Comm 73, 113 (1990), Phys Rev B 41, 2927 (1990) ${ }^{366}$ P H Beton, B R Snell, P C Maın, A Neves, J R Owers-Bradley, L Eaves, M Henını, O H Hughes, S P Beaumont, and C D W Wilkınson, $J$ Phys Condens Matter 1, 7505 (1989) ${ }^{367}$ E Castaño and G Kirczenow, Phys Rev B 41, 5055 (1990) Y Avisha1, M Kaveh, S Shatz, and Y B Band, $J$ Phys Condens Matter 1, 6907 (1989)

${ }^{368}$ C G Smith, M Pepper, R Newbury, H Ahmed, D G Hasko, D C Peacock, J E F Frost,

D A Ritchie, G A C Jones, and G Hill, J Phys Condens Matter 1, 6763 (1989) ${ }^{369}$ Y Hirayama and T Saku, Jpn $J$ Appl Phys (to be publıshed)
} 


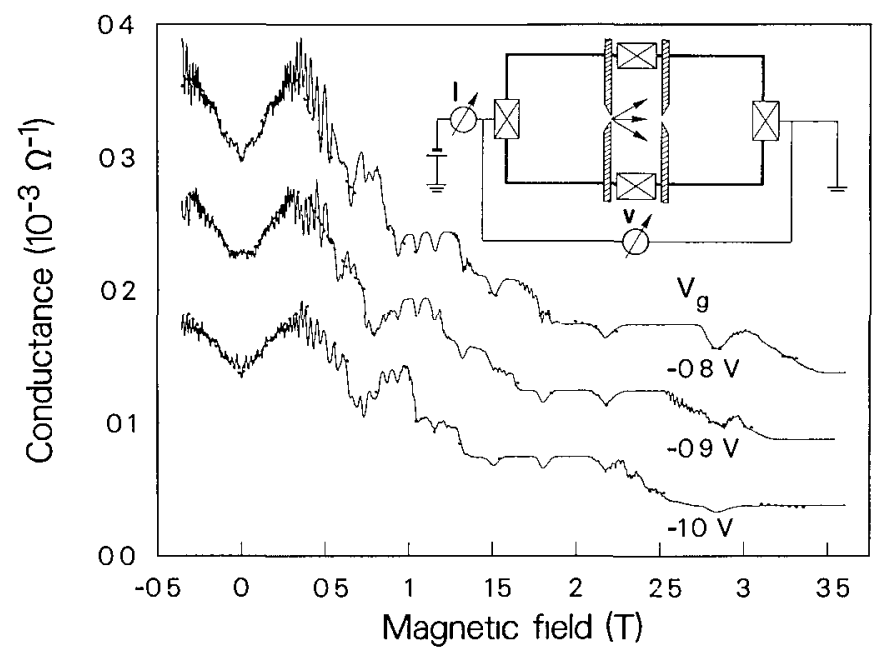

FIG 64 Magnetic field dependence of the series conductance of two opposite point contacts (measured as shown in the inset, the point contact separation is $L=1.0 \mu \mathrm{m}$ ) for three different values of the gate voltage (solid curves) at $T=100 \mathrm{mK}$ For clarity, subsequent curves from bottom to top are offset by $0.5 \times 10^{-4} \Omega^{-1}$, with the lowest curve shown at its actual value. The dotted curves are calculated from Eqs. (15.10) and (10.8), with the point contact width as adjustable parameter Taken from A A M Staring et al, Phys Rev B. 41, 8461 (1990)

The expression for the two-terminal series resistance of two identical opposite point contacts in terms of the direct transmission probability can be obtained from the Landauer-Büttiker formalism, ${ }^{5}$ as was done in Ref. 329. We give here an equivalent, somewhat more intuitive derivation. Consider the geometry shown in Fig. 64 (inset). A fraction $T_{\mathrm{d}} / N$ of the current $G V$ injected through the first point contact by the current source is directly transmitted through the second point contact (and then drained to ground). Here $G=\left(2 e^{2} / h\right) N$ is the conductance of the individual point contact, and $V$ is the source-drain voltage. The remaining fraction $1-T_{\mathrm{d}} / N$ equilibrates in the region between the point contacts, as a result of inelastic scattering (elastic scattering is sufficient if phase coherence does not play a role). Since that region cannot drain charge (the attached contacts are not connected to ground), these electrons will eventually leave via one of the two point contacts. For a symmetric structure we may assume that the fraction $\frac{1}{2}\left(1-T_{\mathrm{d}} / N\right)$ of the injected current $G V$ is transmitted through the second point contact after equilibration. The total source-drain current $I$ is the sum of the direct and indirect contributions:

$$
I=\frac{1}{2}\left(1+T_{\mathrm{d}} / N\right) G V
$$

The series conductance $G_{\text {series }}=I / V$ becomes

$$
G_{\text {series }}=\frac{1}{2} G\left(1+T_{\mathrm{d}} / N\right) \text {. }
$$


In the absence of direct transmission $\left(T_{d}=0\right)$, one recovers the ohmic addition law for the resistance, as expected for the case of complete intervening equilibration (cf the related analysis by Buttiker of tunneling in series barriers ${ }^{370}{ }^{371}$ ) At the opposite extreme, if all transmission is $\operatorname{direct}\left(T_{\mathrm{d}}=N\right)$, the series conductance is identical to that of the single point contact Substituting (153) into Eq (158), we obtain the result ${ }^{329}$ for small but nonzero direct transmission

$$
G_{\text {series }}=\frac{1}{2} G\left(1+f\left(W_{\mathrm{mxx}} / 2 L\right)\right)
$$

The quantized plateaus in the series resistance, observed experimentally, ${ }^{357}$ are of course not obtained in the semiclassical calculation leading to Eq (159) However, since the nonadditivity is essentıally a semiclassical collimation effect, the present analysis should give a reasonably rehable estimate of deviations from additivity for not too narrow point contacts For a comparison with experiments we refer to Refs 307 and 329 A fully quantum mechanical calculation of the series resistance has been carried out numerically by Baranger (reported in Ref 306) for two closely spaced constrictions

So far we have only considered the case of a zero magnetıc field In a weak magnetic field $\left(2 l_{\text {cycl }}>L\right)$ the situation is rather complicated As discussed in detail in Ref 329, there are two competing effects in weak fields On the one hand, the deflection of the electron beam by the Lorentz force reduces the direct transmission probability, with the effect of decreasing the series conductance On the other hand, the magnetic field enhances the indirect transmission, with the opposite effect The result is an initial decrease in the series conductance for small magnetic fields in the case of strong collimation and an increase in the case of weak collumation This is expected to be a relatively small effect compared with the effects at stronger fields that are discussed below

In stronger fields $\left(2 l_{\text {cycl }}<L\right)$, the direct transmission probability vanishes, which greatly simplifies the situation If we assume that all transmission between the opposite point contacts is with intervening equilibration, then the result $1 \mathbf{s}^{329}$

$$
G_{\text {serics }}=\frac{2 e^{2}}{h}\left(\frac{2}{N}-\frac{1}{N_{\text {wide }}}\right)^{-1}
$$

Here $N$ is the ( $B$-dependent) number of occupied subbands in the point contacts, and $N_{\text {wide }}$ is the number of occupied Landau levels in the 2DEG between the point contacts The physical origin of the simple addition rule

${ }^{370} \mathrm{M}$ Butt1ker, Phys Rev B 33, 3020 (1986)

${ }^{371} \mathrm{M}$ Buttıker, IBM J Res Dev 32, 63 (1988) 
(15.10) is additivity of the four-terminal longitudinal resistance (13.7). From this additivity it follows that for $n$ different point contacts in series, Eq. (15.10) generalizes to

$$
\frac{1}{G_{\text {series }}}-\frac{h}{2 e^{2}} \frac{1}{N_{\text {wide }}}=\sum_{i=1}^{n} R_{\mathrm{L}}(i)
$$

where

$$
R_{\mathrm{L}}(i)=\left(\frac{h}{2 e^{2}}\right)\left(\frac{1}{N_{\imath}}-\frac{1}{N_{\text {wrde }}}\right)
$$

is the four-terminal longitudinal resistance of point contact $i$. Equation (15.10) predicts a nonmonotonic $B$-dependence for $G_{\text {serres }}$. This can most easily be seen by disregarding the discreteness of $N$ and $N_{\text {widc }}$. We then have $N_{\mathrm{L}} \approx E_{\mathrm{F}} / \hbar \omega_{\mathrm{c}}$, while the magnetic field dependence of $N$ (for a square-well confining potential in the point contacts) is given by Eq. (10.8). The resulting $B$-dependence of $G_{\text {scres }}$ is shown in Fig. 64 (dotted curves). The nonmonotonic behavior is due to the delayed depopulation of subbands in the point contacts compared with the broad 2DEG. While the number of occupied Landau levels $N_{\text {wide }}$ in the region between the point contacts decreases steadily with $B$ for $2 l_{\text {cycl }}<L$, the number $N$ of occupied subbands in the point contacts remains approximately constant until $2 l_{\mathrm{c}, \min } \approx W_{\min }$, with $l_{\text {c,m?n }} \equiv l_{\text {cycl }}\left(1-E_{\mathrm{c}} / E_{\mathrm{F}}\right)^{1 / 2}$ denoting the cyclotron radius in the point contact region. In this field interval $G_{\text {serics }}$ increases with $B$, according to Eq. (15.10). For stronger fields, depopulation in the point contacts begins to dominate $G_{\text {series }}$, leading finally to a decreasing conductance (as is the rule for single point contacts; see Section 13.b). The peak in $G_{\text {scres }}$ thus occurs at $2 l_{\mathrm{c}, \min } \approx W_{\min }$.

The remarkable camelback shape of $G_{\text {ceries }}$ versus $B$ predicted by Eq. (15.10) has been observed experimentally by Staring et $a l^{372}$ The data are shown in Fig. 64 (solid curves) for three values of the gate voltage $V_{\mathrm{g}}$ at $T=100 \mathrm{mK}$. The measurement configuration is as shown in the inset, with a point contact separation $L=1.0 \mu \mathrm{m}$. The dotted curves in Fig. 64 are the result of a one-parameter fit to the theoretical expression. It is seen that Eq. (15.10) provides a good description of the overall magnetoresistance behavior from low magnetic fields up to the quantum Hall effect regime. The additional structure in the experimental curves has several different origins, for which we refer to the paper by Staring et al ${ }^{372}$ Similar structure in the two-terminal resistance of a single point contact will be discussed in detail in Section 21.

${ }^{372}$ A. A M. Starıng, L. W. Molenkamp, C. W. J. Beenakker, L. P. Kouwenhoven, and C. T. Foxon, Phys. Rev. B. 41, 8461 (1990) 
We emphasize that Eq $(1510)$ is based on the assumption of compietc equilibration of the current-carrying edge states in the region between the point contacts In a quantızing magnetic field, local equilibrium is reached by inter-Landau level scattering If the potential landscape (both in the point contacts themselves and in the 2DEG region in between) varies by less than the Landau level separation $\hbar \omega_{c}$ on the length scale of the magnetic length $(h / e B)^{1 / 2}$, then inter-Landau level scattering is suppressed in the absence of other scattering mechanisms (see Section 18) This means that the transport from one point contact to the other is adiabatic The series conductance is then simply $G_{\text {serics }}=\left(2 e^{2} / h\right) N$ for two identical point contacts $\left[N \equiv \min \left(N_{1}, N_{2}\right)\right.$ for two different point contacts in series] This expression differs from Eq $(1510)$ if a barrier is present in the point contacts, since that causes the number $N$ of occupied Landau levels in the point contact to be less than the number $N_{\text {wide }}$ of occupied levels in the wide 2DEG [In a strong magnetic field, $N \approx\left(E_{\mathrm{F}}-E_{\mathrm{c}}\right) / \hbar \omega_{\mathrm{c}}$, while $\left.N_{\text {wide }} \approx E_{\mathrm{F}} / \hbar \omega_{\mathrm{c}}\right]$ Adiabatic transport in a magnetic field through two point contacts in series has been studied experimentally by Kouwenhoven et $a l^{373}$ and by Main et $a l^{374}$

\section{JUNCTION SCATTERING}

In the regime of diffusive transport, the Hall bar geometry (a straight current-carrying channel with small side contacts for voltage drop measurements) is very convenient, since it allows an independent determination of the various components of the resistivity tensor A downscaled Hall bar was therefore a natural first choice as a geometry to study ballistic transport in a

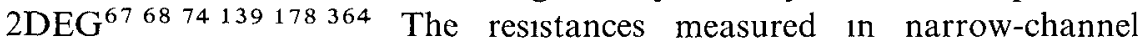
geometries are mainly determined by scattering at the junctions with the side probes ${ }^{289}$ These scattering processes depend strongly on the junction shape This is in contrast to the point contact geometry, compare the very similar results of van Wees et $a l^{6}$ and Wharam et $a l^{7}$ on the quantized conductance of point contacts of a rather different design The strong dependence of the low-field Hall resistance on the junction shape was demonstrated theoretically by Baranger and Stone ${ }^{358}$ and experimentally by Ford et al ${ }^{77}$ and Chang et al ${ }^{375}$ These results superseded many earlier attempts (listed in Ref 360) to explain the discovery by Roukes et al ${ }^{67}$ of the quenching of the Hall effect without modeling the shape of the junction realıstically Baranger and Stone ${ }^{358}$ argued that the rounded corners (present in a realistic situation) at the junction between the main channel and the side branches lead to a

\footnotetext{
${ }^{373}$ L P Kouwenhoven, B J van Wees, W Kool, C J P M Harmans, A A M Staring, and C T Foxon, Phys Rev B 40, 8083 (1989)

${ }^{374} \mathrm{P}$ C Main, P H Beton, B R Snell, A J M Neves, J R Owers-Bradley, L Eaves, S P Beaumont, and C D W Wilkınson, Phys Rev B 40, 10033 (1989)

${ }^{375}$ A M Chang, T Y Chang, and H U Baranger, Phys Rev Lett 63, 996 (1989)
} 
suppression (quenching) of the Hall resistance at low magnetic fields as a consequence of the horn collimation effect discussed in Section 15 a A Hall bar with straight corners, in contrast, does not show a generic suppression of the Hall resistance, ${ }^{376-378}$ although quenching can occur for special parameter values if only a few subbands are occupied in the channel

The quenched Hall effect 6777375379 1s just one of a whole variety of magnetoresistance anomalies observed in narrow Hall bars Other anomalies are the last Hall plateau, ${ }^{676877139178379}$ remınıscent of quantum Hall plateaus, but occurring at much lower fields, the negative Hall resistance ${ }^{77}$ as if the carriers were holes rather than electrons, the bend resistance, ${ }^{289306364380}$ a longitudinal resistance associated with a current bend, which is negative at small magnetic fields and zero at large fields, with an overshoot to a positive value at intermediate fields, and more

In Refs 359 and 360 we have shown that all these phenomena can be qualitatively explained in terms of a few simple semiclassical mechanisms (reviewed in Section 16a) The effects of quantum interference and of quantization of the lateral motion in the narrow conductor are not essential These magnetoresistance anomalies can thus be characterized as classical magneto size effects in the ballistic regime In Section 5, we have discussed classical size effects in the quasi-ballistic regime, where the mean free path is larger than the channel width but smaller than the separation between the voltage probes In that regime, the size effects found in a $2 D E G$ were known from work on metal films and wires ${ }^{102}$ These earher investigations had not anticipated the diversity of magnetoresistance anomalies due to junction scattering in the ballistic regime That is not surprising, considering that the theoretical formalism to describe a resistance measurement within a mean free path had not been developed in that context Indeed, this LandauerButtıker formalism (described in Section 12) found one of its earliest applications ${ }^{268}$ in the context of the quenching of the Hall effect, and the success with which the experimental magnetoresistance anomalies can be described by means of this formalism forms strong evidence for its validity

\section{a Mechanisms}

The variety of magnetoresistance anomalies mentioned can be understood in terms of a few simple characteristics of the curved trajectories of electrons in a classical billiard in the presence of a perpendicular magnetic field ${ }^{359} 360$

\footnotetext{
${ }^{376}$ D G Ravenhall, H W Wyld, and R L Schult, Phys Rev Lett 62,1780 (1989), R L Schult,

H W Wyld, and D G Ravenhall, Phys Rev B 41, 12760 (1990)

${ }^{377}$ G Kirczenow, Phys Rev Lett 62, 2993 (1989), Phys Rev B 42, 5375 (1990)

${ }^{378} \mathrm{H}$ Akera and T Ando, Surf Scl 229, 268 (1990)

${ }^{379} \mathrm{C}$ J B Ford, T J Thornton, R Newbury, M Pepper, H Ahmed, D C Peacock, D A

Ritchie, J E F Frost, and G A C Jones, Phys Rev B 38, 8518 (1988)

${ }^{380} \mathrm{Y}$ Avishai and Y B Band, Phys Rev Lett 62, 2527 (1989)
} 

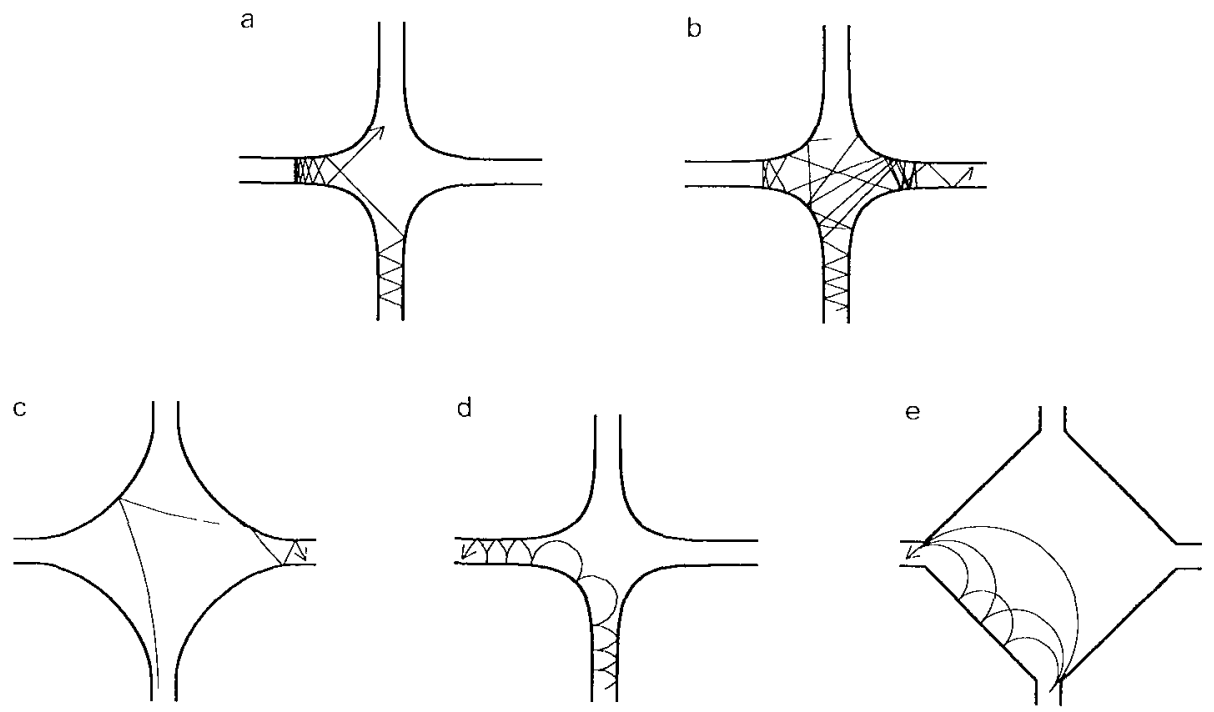

Fig 65 Classical trajectories in an electron bilhard, illustrating the collimation (a), scrambling (b), rebound (c), magnetıc guiding (d) and electron focusing (e) effects. Taken from C. W. J. Beenakker and $\mathbf{H}$ van Houten, in "Electrontc Properties of Multilayers and Low-Dimensional Semiconductor Structures" (J. M Chamberlain, L. Eaves, and J C. Portal, eds.). Plenum, London 1990

At very small magnetic fields, collimation and scrambling are the key concepts. The gradual widening of the channel on approaching the junction reduces the injection-acceptance cone, which is the cone of angles with the channel axis within which an electron is injected into the junction or within which an electron can enter the channel coming from the junction. This is the horn collimation effect ${ }^{329}$ discussed in Section 15.a (see Fig. 65a). If the injection-acceptance cone is smaller than $90^{\circ}$, then the cones of two channels at right angles do not overlap. That means that an electron approaching the side probe comıng from the main channel will be reflected (Fig. 65a) and will then typically undergo multuple reflections in the junction region (Fig. 65b). The trajectory is thus scrambled, whereby the probability for the electron to enter the left or right side probe in a weak magnetic field is equalized. This suppresses the Hall voltage. This "scrambling" mechanism for the quenching of the Hall effect requires a weaker collimation than the "nozzle" mechanism put forward by Baranger and Stone ${ }^{358}$ (we return to both these mechanisms in Section 16.c). Scrambling is not effective in the geometry shown in Fig. 65c, in which a large portion of the boundary in the junction is oriented at approximately $45^{\circ}$ with the channel axis. An electron reflected from a side 
probe at this boundary has a large probability of entering the opposite side probe This is the "rebound" mechanism for a negative Hall resistance proposed by Ford et al ${ }^{77}$

At somewhat larger magnetic fields, guiding takes over As illustrated in Fig 65d, the electron is guided by the magnetic field along equipotentials around the corner Guiding is fully effective when the cyclotron radius $l_{\text {cycl }}$ becomes smaller than the minimal radius of curvature $r_{\min }$ of the cornerthat 1s, for magnetic fields greater than the guiding field $B_{\mathrm{g}} \equiv \hbar k_{\mathrm{F}} / e r_{\min }$ In the regime $B \gtrsim B_{\mathrm{g}}$ the junction cannot scatter the electron back into the channel from which it came The absence of backscattering in this case is an entirely classical, weak-field phenomenon (cf Section 13 b) Because of the absence of backscattering, the longitudinal resistance vanishes, and the Hall resistance $R_{\mathrm{H}}$ becomes equal to the contact resistance of the channel, just as in the quantum Hall effect, but without quantization of $R_{\mathrm{H}}$ The contact resistance $R_{\text {coniact }} \approx\left(h / 2 e^{2}\right)\left(\pi / k_{\mathrm{F}} W\right)$ is approximately independent of the magnetic field for fields such that the cyclotron diameter $2 l_{\text {cycl }}$ is greater than the channel width $W$, that is, for fields below $B_{\text {crit }} \equiv 2 \hbar k_{\mathrm{F}} / e W$ (see Sections 12 and 13) This explains the occurrence of the "last plateau" in $R_{\mathrm{H}}$ for $B_{\mathrm{g}} \lesssim B \lesssim B_{\text {crit }}$ as a classical effect At the low-field end of the plateau, the Hall resistance is sensitive to geometrical resonances that increase the fraction of electrons guided around the corner into the side probe Figure 65e 1llustrates the occurrence of one such a geometrical resonance as a result of the magnetic focusing of electrons into the side probe, at magnetic fields such that the separation of the two perpendicular channels is an integer multiple of the cyclotron diameter This is in direct analogy with electron focusing in a double-point contact geometry (see Section 14) and leads to periodic oscillations superimposed on the Hall plateau Another geometrical resonance with similar effect is discussed in Ref 360

These mechanisms for oscillations in the resistance depend on a commensurability between the cyclotron radius and a characteristic dimension of the junction, but do not involve the wavelength of the electrons as an independent length scale This distinguishes these geometrical resonances conceptually from the quantum resonances due to bound states in the Junction considered in Refs 376, 377, and 380-382

\section{b Magnetoresistance Anomalles}

In this subsection we compare, following Ref 360 , the semiclassical theory with representative experiments on laterally confined two-dimensional

\footnotetext{
${ }^{381} \mathrm{G}$ Kirczenow, Sold State Comm 71, 469 (1989)

${ }^{382}$ F M Peeters, in Ref 16, Phys Rev Lett 61, 589 (1988), Superlattices and Microstructures 6, $217(1989)$
} 


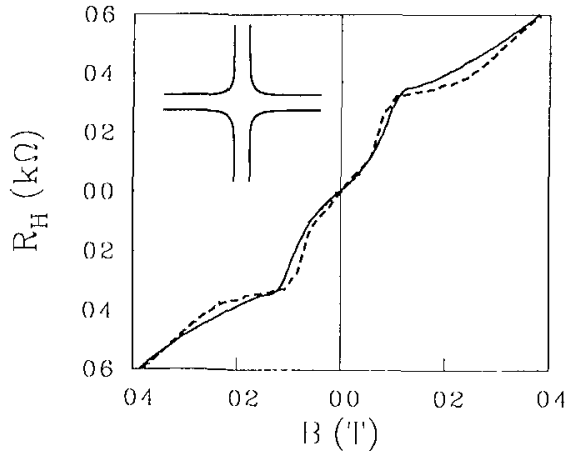

FIG. 66. Hall resistance as measured (solid curve) by Simmons et al. ${ }^{178}$, and as calculated dashed curve) for the hard-wall geometry in the inset ( $W=0.8 \mu \mathrm{m}$ and $E_{\Gamma}=14 \mathrm{meV}$ ). The dotted line is $R_{\mathrm{II}}$ in a bulk 2DEG. Taken from C. W. J. Beenakker and H. van Houten in "Electronic Properties of Multilayers and Low-Dimensional Semiconductor Structures," (J. M. Chamberlain, L. Eaves, and J. C. Portal, eds.). Plenum, London, 1990.

electron gases in high-mobility GaAs-AlGaAs heterostructures. The calculations are based on a simulation of the classical trajectories of a large number (typically $10^{4}$ ) of electrons with the Fermi energy, to determine the classical transmission probabilities. The resistances then follow from the Büttiker formula (12.12). We refer to Refs. 359 and 360 for details on the method of calculation. We first discuss the Hall resistance $R_{\mathrm{H}}$.

Figure 66 shows the precursor of the classical Hall plateau (the "last plateau") in a relatively wide Hall cross. The experimental data (solid curve) is from a paper by Simmons et al. ${ }^{178}$ The semiclassical calculation (dashed curve) is for a square-well confining potential of channel width $W=0.8 \mu \mathrm{m}$ (as estimated in the experimental paper) and with the relatively sharp corners shown in the inset. The Fermi energy used in the calculation is $E_{\mathrm{F}}=14 \mathrm{meV}$, which corresponds (via $n_{\mathrm{s}}=E_{\mathrm{F}} m / \pi \hbar^{2}$ ) to a sheet density in the channel of $n_{\mathrm{s}}=3.9 \times 10^{15} \mathrm{~m}^{-2}$, somewhat below the value of $4.9 \times 10^{15} \mathrm{~m}^{-2}$ of the bulk material in the experiment. Good agreement between theory and experiment is seen in Fig. 66. Near zero magnetic field, the Hall resistance in this geometry is close to the linear result $R_{\mathrm{H}}=B / e n_{\mathrm{s}}$ for a bulk 2DEG (dotted line). The corners are sufficiently smooth to generate a Hall plateau via the guiding mechanism discussed in Section 16.a. The horn collimation effect, however, is not sufficiently large to suppress $R_{\mathrm{H}}$ at small $B$. Indeed, the injection-acceptance cone for this junction is considerably wider (about $115^{\circ}$ ) than the maximal angular opening of $90^{\circ}$ required for quenching of the Hall effect via the scrambling mechanism described in Section 16.a.

The low-field Hall resistance changes drastically if the channel width becomes smaller, relative to the radius of curvature of the corners. Figure 67a shows experimental data by Ford et al. ${ }^{77}$ The solid and dotted curves are for the geometries shown respectively in the upper left and lower right insets of Fig. 67a. Note that these insets indicate the gates with which the Hall crosses are defined electrostatically. The equipotentials in the 2DEG will be smoother than the contours of the gates. The experiment shows a well- 
(a)

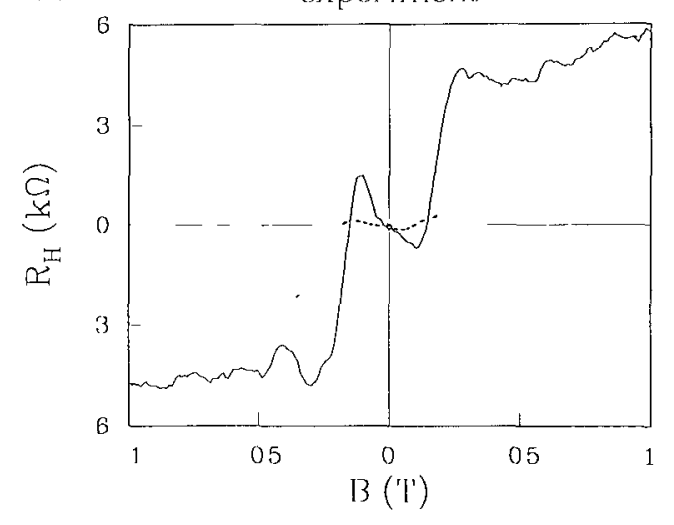

(b)

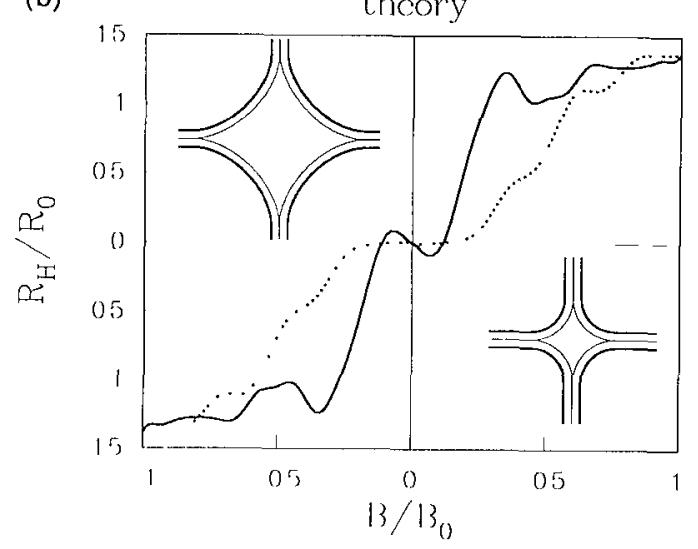

FiG. 67. Hall resistance as measured (a) by Ford et al. ${ }^{77}$ and as calculated (b). In (a) as well as in (b), the solid curve corresponds to the geometry in the upper left inset, the dotted curve to the geometry in the lower right inset. The insets in (a) indicate the shape of the gates, not the actual confining potential. The insets in (b) show equipotentials of the confining potential at $E_{\mathrm{F}}$ (thick contour) and 0 (thin contour). The potential rises parabolically from 0 to $E_{\mathrm{F}}$, and vanishes in the diamond-shaped region at the center of the junction. Taken from C. W. J. Beenakker and H. van Houten, in "Electronic Properties of Multilayers and Low-Dimensional Semiconductor Structures" (J. M. Chamberlain, L. Eaves, and J. C. Portal, eds.). Plenum, London, 1990.

developed Hall plateau with superimposed fine structure. At small positive fields $R_{\mathrm{H}}$ is either quenched or negative, depending on the geometry. The geometry is seen to affect also the width of the Hall plateau but not the height. In Fig. 67b we give the results of the semiclassical theory for the two geometries in the insets, which should be reasonable representations of the 
(a)

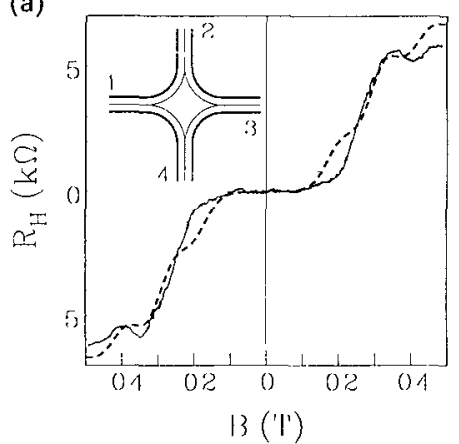

(b)

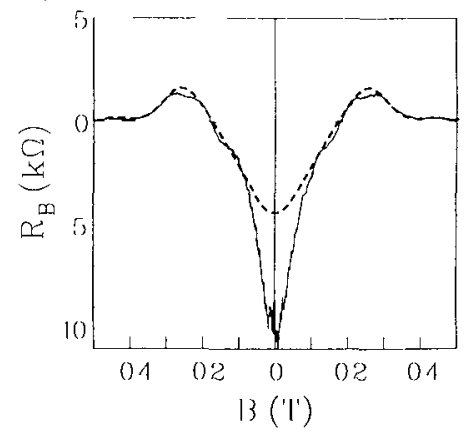

FIG. 68. Hall resistance $R_{11} \equiv R_{13,24}$ (a) and bend resistance $R_{\mathrm{B}} \equiv R_{12,43}$ (b), as measured (solid curves) by Timp et al. ${ }^{306}$ and as calculated (dashed curves) for the geometry in the inset (consisting of a parabolic confining potential with the equipotentials at $E_{\mathrm{F}}$ and 0 shown respectively as thick and thin contours; the parameters are $W=100 \mathrm{~nm}$ and $E_{\mathrm{F}}=3.9 \mathrm{meV}$ ). The dotted line in (a) is $R_{\mathrm{H}}$ in a bulk 2DEG. Taken from C. W. J. Beenakker and H. van Houten, in "Electronic Properties of Multilayers and Low-Dimensional Semiconductor Structures" (J. M. Chamberlain, L. Eaves, and J. C. Portal, eds.). Plenum, London, 1990.

confining potential induced by the gates in the experiment. In the theoretical plot the resistance and the magnetic field are given in units of

$$
R_{0} \equiv \frac{h}{2 e^{2}} \frac{\pi}{k_{\mathrm{F}} W}, \quad B_{0} \equiv \frac{\hbar k_{\mathrm{F}}}{e W},
$$

where the channel width $W$ for the parabolic confinement used is defined as the separation of the equipotentials at the Fermi energy ( $W_{\text {par }}$ in Section 10$)$. The experimental estimates $W \approx 90 \mathrm{~nm}, \quad n_{\mathrm{s}} \approx 1.2 \times 10^{15} \mathrm{~m}^{-2}$ imply $R_{0}=5.2 \mathrm{k} \Omega, B_{0}=0.64 \mathrm{~T}$. With these parameters the calculated resistance and field scales do not agree well with the experiment, which may be due in part to the uncertainties in the modeling of the shape of the experimental confining potential. The $\pm B$ asymmetry in the experimental plot is undoubtedly due to asymmetries in the cross geometry [in the calculation the geometry has fourfold symmetry, which leads automatically to $\left.R_{\mathrm{H}}(B)=-R_{\mathrm{H}}(-B)\right]$. Apart from these differences, there is agreement in all the important features: the appearance of quenched and negative Hall resistances, the independence of the height of the last Hall plateau on the smoothness of the corners, and the shift of the onset of the last plateau to lower fields for smoother corners. The oscillations on the last plateau in the calculation (which, as we discussed in Section 16.a, are due to geometrical resonances) are also quite similar to those in the experiment, indicating that these are classical rather than quantum resonances.

We now turn to the bend resistance $R_{\mathrm{B}}$. In Fig. 68 we show experimental 
data by Timp et al. ${ }^{306}$ (solid curves) on $R_{\mathrm{B}} \equiv R_{12,43}$ and $R_{\mathrm{H}} \equiv R_{13,24}$ measured in the same Hall cross (defined by gates of a shape similar to that in the lower right inset of Fig. 67a; see the inset of Fig. 68a for the numbering of the channels). The dashed curves are calculated for a parabolic confining potential in the channels (with the experimental values $W=100 \mathrm{~nm}$, $E_{\mathrm{F}}=3.9 \mathrm{meV}$ ) and with corners as shown in the inset of Fig. $68 \mathrm{a}$. The calculated quenching of the Hall resistance and the onset of the last plateau are in good agreement with the experiment, and also the observed overshoot of the bend resistance around $0.2 \mathrm{~T}$ as well as the width of the negative peak in $R_{\mathrm{B}}$ around zero field are well described by the calculation. The calculated height of the negative peak, however, is too small by more than a factor of 2 . We consider this disagreement to be significant in view of the quantitative agreement with the other features in both $R_{\mathrm{B}}$ and $R_{\mathrm{H}}$. The negative peak in $R_{\mathrm{B}}$ is due to the fact that the collimation effect couples the current source 1 more strongly to voltage probe 3 than to voltage probe 4 , so $R_{\mathrm{B}} \propto V_{4}-V_{3}$ is negative for small magnetic fields (at larger fields the Lorentz force destroys collimation by bending the trajectories, so $R_{\mathrm{B}}$ shoots up to a positive value until guiding takes over and brings $R_{\mathrm{B}}$ down to zero by eliminating backscattering at the junction). The discrepancy in Fig. $68 \mathrm{~b}$ thus seems to indicate that the semiclassical calculation underestimates the collimation effect in this geometry. The positive overshoot of $R_{\mathrm{B}}$ seen in Fig. $68 \mathrm{~b}$ is found only for rounded corners. This explains the near absence of the effect in the calculation of Kirczenow ${ }^{381}$ for a junction with straight corners.

For a discussion of the temperature dependence of the magnetoresistance anomalies, we refer to Ref. 360 . Here it suffices to note that the experiments discussed were carried out at temperatures around $1 \mathrm{~K}$, for which we expect the zero-temperature semiclassical calculation to be appropriate. At lower temperatures the effects of quantum mechanical phase coherence that have been neglected will become more important. ${ }^{195}$ At higher temperatures the thermal average smears out the magnetoresistance anomalies and eventually inelastic scattering causes a transition to the diffusive transport regime in which the resistances have their normal $B$-dependence.

\section{c. Electron Waveguide versus Electron Billiard}

The overall agreement between the experiments and the semiclassical calculations is remarkable in view of the fact that the channel width in the narrowest structures considered is comparable to the Fermi wavelength. When the first experiments on these "electron waveguides" appeared, it was expected that the presence of only a small number of occupied transverse waveguide modes would fundamentally alter the nature of electron transport. ${ }^{68}$ The results of Refs. 359 and 356 show instead that the modal structure plays only a minor role and that the magnetoresistance anomalies observed 
are characteristic for the classical ballistic transport regime The reason that a phenomenon such as the quenching of the Hall effect has been observed only in Hall crosses with narrow channels is simply that the radius of curvature of the corners at the junction is too small compared with the channel width in wider structures This is not an essential limitation, and the various magnetoresistance anomalies discussed here should be observable in macroscopic Hall bars with artificially smoothed corners, provided of course that the dimensions of the junction remain well below the mean free path Ballistic transport is essential, but a small number of occupied modes is not

Although we believe that the characteristic features of the magnetoresistance anomalies are now understood, several interesting points of disagreement between theory and experiment remain that merit further investigation One of these is the discrepancy in the magnitude of the negative bend resistance at zero magnetic field noted before The disappearance of a region of quenched Hall resistance at low electron density is another unexpected observation by Chang et $a l^{375}$ and Roukes et al ${ }^{383}$ The semiclassical theory predicts a universal behavior (for a given geometry) if the resistance and magnetic field are scaled by $R_{0}$ and $B_{0}$ defined in Eq (161) For a square-well confining potential the channel width $W$ is the same at each energy, and since $B_{0} \propto k_{\mathrm{F}}$ one would expect the field region of quenched Hall resistance to vary with the electron density as $\sqrt{n_{\mathrm{s}}}$ For a more realistic smooth confining potential, $W$ depends on $E_{\mathrm{F}}$ and thus on $n_{\mathrm{s}}$ as well, in a way that is difficult to estımate reliably In any case, the experiments point to a systematic disappearance of the quench at the lowest densities, which is not accounted for by the present theory (and has been attributed by Chang et $a^{375}$ to enhanced diffraction at low electron density as a result of the increase in the Fermı wavelength) For a detalled investigation of departures from classical scalıng, we refer to a paper by Roukes et $a l^{384}$ As a third point, we mention the curious density dependence of the quenching observed in approximately straight junctions by Roukes et al,${ }^{383}$ who find a low-field suppression of $R_{\mathrm{H}}$ that occurs only at or near certain specific values of the electron density The semiclassical model applied to a straight Hall cross (either defined by a square well or by a parabolic confining potential) gives a low-field slope of $R_{\mathrm{H}}$ close to its bulk $2 \mathrm{D}$ value The fully quantum mechanical calculations for a straight junction ${ }^{376} 381$ do give quenching at special parameter values, but not for the many-mode channels in this experiment (in which quenching occurs with as many as 10 modes occupied, whereas in the calculations a straight cross with more than 3 occupred modes in the channel does not show a quench)

${ }^{383} \mathrm{M}$ L Roukes, $\mathrm{T}$ J Thornton, A Scherer, J A Simmons, B P van der Gaag, and E D Beebe, in $\operatorname{Ref} 16$

${ }^{384} \mathrm{M}$ L Roukes, A Scherer, and B P van der Gaag, Phys Rev Lett 64, 1154 (1990) 
In addition to the points of disagreement discussed, there are fine details in the measured magnetoresistances, expecially at the lowest temperatures (below $100 \mathrm{mK}$ ), which are not obtained in the semiclassical approximation The quantum mechanical calculations ${ }^{358} 376377381$ show a great deal of fine structure due to interference of the waves scattered by the junction The fine structure in most experiments is not quite as pronounced as in the calculations presumably partly as a result of a loss of phase coherence after many multiple scatterings in the junction The limited degree of phase coherence in the experiments and the smoothing effect of a finite temperature help to make the semiclassical model work so well even for the narrowest channels We draw attention to the fact that classical chaotic scattering can also be a source of irregular resistance fluctuations (see Ref 360)

Some of the most pronounced features in the quantum mechanical calculations are due to transmission resonances that result from the presence of bound states in the junction ${ }^{376377380-382}$ In Section 16 a we have discussed a different mechanism for transmission resonances that has a classical, rather than a quantum mechanical, origin As mentioned in Section $16 \mathrm{~b}$, the oscillations on the last Hall plateau observed experimentally are quite well accounted for by these geometrical resonances One way to distinguish experimentally between these resonance mechanisms is by means of the temperature dependence, which should be much weaker for the classical than for the quantum effect One would thus conclude that the fluctuations in Fig 67a, measured by Ford et al ${ }^{77}$ at $42 \mathrm{~K}$, have a classical origin, while the fine structure that Ford et al ${ }^{385}$ observe only at $\mathrm{mK}$ temperatures (see below) is intrinsically quantum mechanical

The differences between the semiclassical and the quantum mechanical models may best be illustrated by considering once again the quenching of the Hall effect, which has the most subtle explanation and is the most sensitive to the geometry among the magnetoresistance anomalies observed in the ballistic regime The classical scrambling of the trajectories after multiple reflections suppresses the asymmetry between the transmission probabilities $t_{l}$ and $t_{\mathrm{r}}$ to enter the left or right voltage probe, and without this transmission asymmetry there can be no Hall voltage We emphasize that this scrambling mechanism is consistent with the original findings of Baranger and Stone ${ }^{358}$ that quenching requires collimation The point is that the collimation effect leads to nonoverlapping injection-acceptance cones of two perpendicular channels, which ensures that electrons cannot enter the voltage probe from the current source directly, but rather only after multiple reflections (cf Section 16 a) In this way a rather weak collimation to within an injection-acceptance cone of about $90^{\circ}$ angular opening is sufficient to

${ }^{385}$ C J B Ford, S Washburn, M Buttıker, C M Knoedler, and J M Hong, Surf Scl 229, 298 (1990) 
induce a suppression of the Hall resistance via the scrambling mechanism

Collımation can also suppress $R_{\mathrm{H}}$ directly by strongly reducing $t_{l}$ and $t_{\mathrm{r}}$ relative to $t_{\mathrm{c}}$ (the probability for transmission straight through the junction) This nozzle mechanism, introduced by Baranger and Stone, ${ }^{358}$ requires a strong collimation of the injected beam in order to affect $R_{\mathrm{H}}$ appreciably In the geometries considered here, we find that quenching of $R_{\mathrm{H}}$ is due predominantly to scrambling and not to the nozzle mechanism $\left(t_{l}\right.$ and $t_{\mathrm{r}}$ each remain more than $30 \%$ of $t_{\mathrm{s}}$ ), but data by Baranger and Stone ${ }^{358}$ show that both mechanisms can play an important role

There is a third proposed mechanism for the quenching of the Hall effect, ${ }^{376} 377$ which is the reduction of the transmission asymmetry due to a bound state in the junction The bound state mechanism is purely quantum mechanical and does not require collimation (in contrast to the classical scrambling and nozzle mechanisms) Numerical calculations have shown that it is only effective in straight Hall crosses with very narrow channels (not more than three modes occupied), and even then for special values of the Fermı energy only Although this mechanism cannot account for the experiments performed thus far, it may become of importance in future work A resonant suppression of the Hall resistance may also occur in strong magnetic fields, in the regime where the Hall resistance in wide Hall crosses would be quantized Such an effect is intimately related to the high-field Aharonov-Bohm magnetoresistance oscillations in a singly connected geometry (see Section 21) Ford et al ${ }^{385}$ have observed oscillations superımposed on quantized Hall plateaux at low temperatures in very narrow crosses of two different shapes (see Fig 69) The strong temperature dependence indicates that these oscillations are resonances due to the formation of bound states in the cross 306385386

\section{TunNeling}

In this section we review recent experiments on tunneling through potential barriers in a two-dimensional electron gas Subsection 17.a deals with resonant tunneling through a bound state in the region between two barriers Resonant tunneling has previously been studied extensively in layered semiconductor heterostructures for transport perpendicular to the layers ${ }^{387-389}$ For example, a thin AlGaAs layer embedded between two GaAs layers forms a potential barrier, whose height and width can be tallored

${ }^{386} \mathrm{M}$ Buttıker, in Ref 9

${ }^{387} \mathrm{R}$ Tsu and L Esakı, Appl Phys Lett 22, 562 (1973)

${ }^{388}$ L L Chang, L Esakı, and R Tsu, Appl Phys Lett 24, 593 (1974)

${ }^{389} \mathrm{E}$ S Alves, L Eaves, M Henını, O H Hughes, M L Leadbeater, F W Sheard, and G A Toombs, Electron Lett 24, 1190 (1988) 


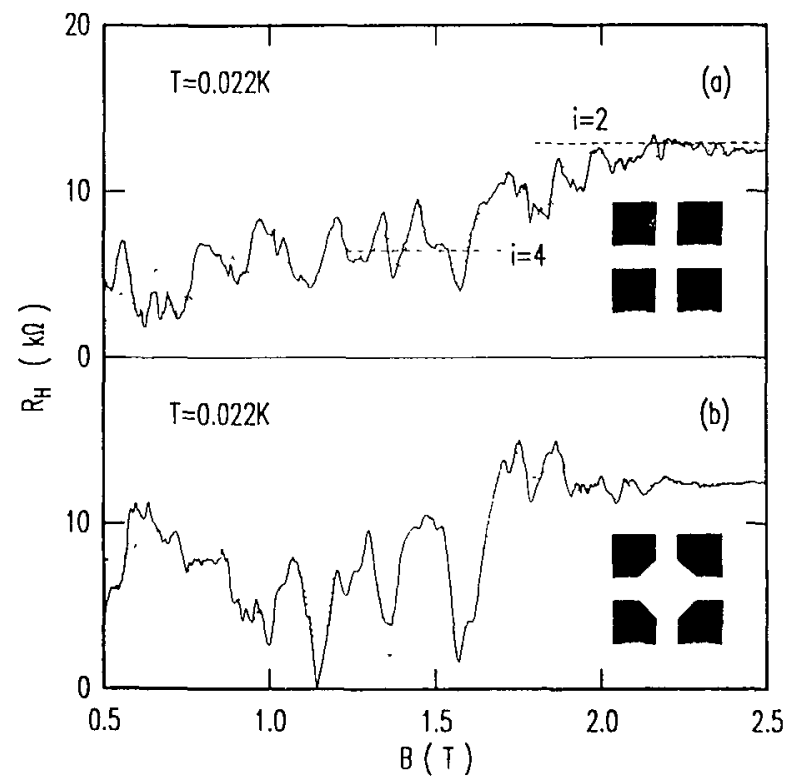

Fig. 69. Measured Hall resistance in an abrupt (a) and in a widened (b) cross as a function of $B$ in the strong field regime. Large fluctuations are resolved at the low temperature of $22 \mathrm{mK}$. The dotted curves indicate the reproducibility of the measurement. Taken from C. J. B. Ford $e$ t al., Surf. Sci. 229, 298 (1990).

with great precision by means of advanced growth techniques (such as molecular beam epitaxy). Because of the free motion in the plane of the layers, one can only realize bound states with respect to one direction. Tunneling resonances are consequently smeared out over a broad energy range. A 2DEG offers the possibility of confinement in all directions and thus of a sharp resonance. A gate allows one to define potential barriers of adjustable height in the 2DEG. In contrast, the heterostructure layers form fixed potential barriers, so one needs to study a current-voltage characteristic to tune the system through a resonance (observable as a peak in the $I-V$ curve). The gate-induced barriers in a $2 \mathrm{DEG}$ offer a useful additional degree of freedom, allowing a study of resonant tunneling in the linear response regime of small applied voltages (to which we limit the discussion in this review). A drawback of these barriers is that their shape cannot be precisely controlled, or modeled, so that a description of the tunneling process will of necessity be qualitative.

Subsection 17.b deals with the effects of Coulomb repulsion on tunneling in a 2DEG. The electrostatic effects of charge buildup in the 1D potential well formed by heterostructure layers have received considerable attention in 
recent years ${ }^{389} 390$ Because of the large capacitance of the potential well in this case (resulting from the large surface area of the layers) these are macroscopic effects, involving a large number of electrons The 3D potential well in a $2 D E G$ nanostructure, in contrast, can have a very small capacitance and may contain a few electrons only The tunneling of a single electron into the well will then have a considerable effect on the electrostatic potential difference with the surrounding 2DEG For a small applied voltage this effect of the Coulomb repulsion can completely suppress the tunneling current In metals this "Coulomb blockade" of tunneling has been studied extensive$1 y^{391}$ In those systems a semiclassical description suffices The large Fermi wavelength in a 2DEG should allow the study of quantum mechanical effects on the Coulomb blockade or, more generally, of the interplay between electron-electron interactions and resonant tunneling 318392393

\section{a Resonant Tunneling}

The simplest geometry in which one might expect to observe transmission resonances is formed by a single potential barrier across a 2DEG channel Such a geometry was studied by Washburn et al ${ }^{394}$ in a GaAs-AlGaAs heterostructure containıng a $2-\mu \mathrm{m}$-wide channel with a 45 -nm-long gate on top of the heterostructure At low temperatures (around $20 \mathrm{mK}$ ) an irregular set of peaks was found in the conductance as a function of gate voltage in the region close to the depletion threshold The amplitude of the peaks was on the order of $e^{2} / h$ The origin of the effect could not be pinned down The authors examine the possibility that transmission resonances associated with a square potential barrier are responsible for the oscillations in the conductance, but also note that the actual barrier is more likely to be smooth on the scale of the wavelength For such a smooth barrier the transmission probability as a function of energy does not show oscillations It seems most likely that the effect is disorder-related Davies and Nixon ${ }^{395}$ have suggested that some of the structure observed in this experiment could be due to potential fluctuations in the region under the gate These fluctuations can be rather pronounced close to the depletion threshold, due to the lack of screening in the low-density electron gas A quantum mechanical calculation of transmission through such a fluctuating barrier has not been performed As discussed below, conductance peaks of order $e^{2} / h$ occur in the case of

\footnotetext{
${ }^{390}$ A Zasldvsky, V J Goldman, D C Tsuı and J E Cunnungham, Appl Phys Lett 53, 1408 (1988)

${ }^{391} \mathrm{~K}$ K Likharev, IBM J Res Dev 32, 144 (1988)

${ }^{392} \mathrm{~K} \mathrm{Ng}$ and P A Lee, Phys Rev Lett 61, 1768 (1988)

${ }^{393} \mathrm{~L}$ I Glazman and K A Matveev, Pls'ma Zh Eksp Teor Flz 48, 403 (1988) [JETP Lett 48, 445 (1988)]

${ }^{394} \mathrm{~S}$ Washburn, A B Fowler, H Schmid, and D Kern, Phys Rev B 38, 1554 (1988)

395J H Davies, Semicond Scl Technol 3, 995 (1988) See also Ref 72
} 
resonant tunneling via localized states in the barrier (associated with impurities), a mechanism that might well play a role in the experiment of Washburn et al ${ }^{394}$

In pursuit of resonant tunnelıng in a $2 \mathrm{DEG}$, Chou et al $^{396}$ have fabricated double-barrier devices involving two closely spaced short gates across a wide $\mathrm{GaAs}$ - AlGaAs heterostructure Both the spacing and the length of the gates were $100 \mathrm{~nm}$ They observed a peak in the transconductance (the derivative of the channel current with respect to the gate voltage), which was attributed to resonant tunneling through a quasi-bound state in the $2 \mathrm{D}$ potential well between the barriers Palevsk1 et al ${ }^{397}$ have also investigated transport through two closely spaced potential barriers in a double-gate structure, but they did not find evidence for transmission resonances

A 3D potential well has truly bound states and is expected to show the strongest transmission resonances Transport through such a cavity or "quantum box" has been studied theoretically by several authors 318333382398 Experıments have been performed by Smith et al $^{399-401}$ Their device is based on a quantum point contact, but contains two potential barriers that separate the constriction from the wide $2 \mathrm{DEG}$ regions (see the inset of Fig 70) As the negative gate voltage is increased, a potential well is formed between the two barriers, resulting in confinement in all directions The tunneling regime corresponds to a resistance $R$ that is greater than $h / 2 e^{2}$ It is also possible to study the ballistıc regime $R<h / 2 e^{2}$ when the height of the potential barriers is less than the Fermi energy In this regime the transmission resonances are similar to the resonances in long quantum point contacts (these are determined by an interplay of tunneling through evanescent modes and reflection at the entrance and exit of the point contact, cf Section 13) Results of Smith et al ${ }^{399} 401$ for the resistance as a function of gate voltage at $330 \mathrm{mK}$ are reproduced in $\mathrm{F}_{1} \mathrm{~g} 70$ In the tunneling regime $\left(R>h / 2 e^{2}\right)$ giant resistance oscillations are observed $\mathrm{A}$ regular series of smaller resistance peaks is found in the ballistic regime $\left(R<h / 2 e^{2}\right)$ MartinMoreno and Smith ${ }^{33}$ have modeled the electrostatıc potential in the device of Refs 399-401 and have performed a quantum mechanical calculation of

\footnotetext{
${ }^{396}$ S Y Chou, D R Allee, R F W Pease, and J S Harris, Jr, Appl Phys Lett 55, 176 (1989) ${ }^{397}$ A Palevskı, M Heıblum, C P Umbach, C M Knoedler, A N Broers, and R H Koch, Phys Rev Lett 62, 1776 (1989)

${ }^{398}$ Y Avishai and Y B Band, Phys Rev B 41, 3253 (1990)

${ }^{399} \mathrm{C}$ G Smith, M Pepper, H Ahmed, J E Frost, D G Hasko, D C Peacock, D A Ritchie, and G A C Jones, Superlattices and Microstructures 5, 599 (1989)

${ }^{400}$ C G Smith, M Pepper, H Ahmed, J E F Frost, D G Hasko, D C Peacock, D A Ritchie, and G A C Jones, $J$ Phys C 21, L893 (1988)

${ }^{401}$ C G Smith, M Pepper, H Ahmed, J E F Frost, D G Hasko, D A Ritchie, and G A C Jones, Surf Sct 228, 387 (1990)
} 


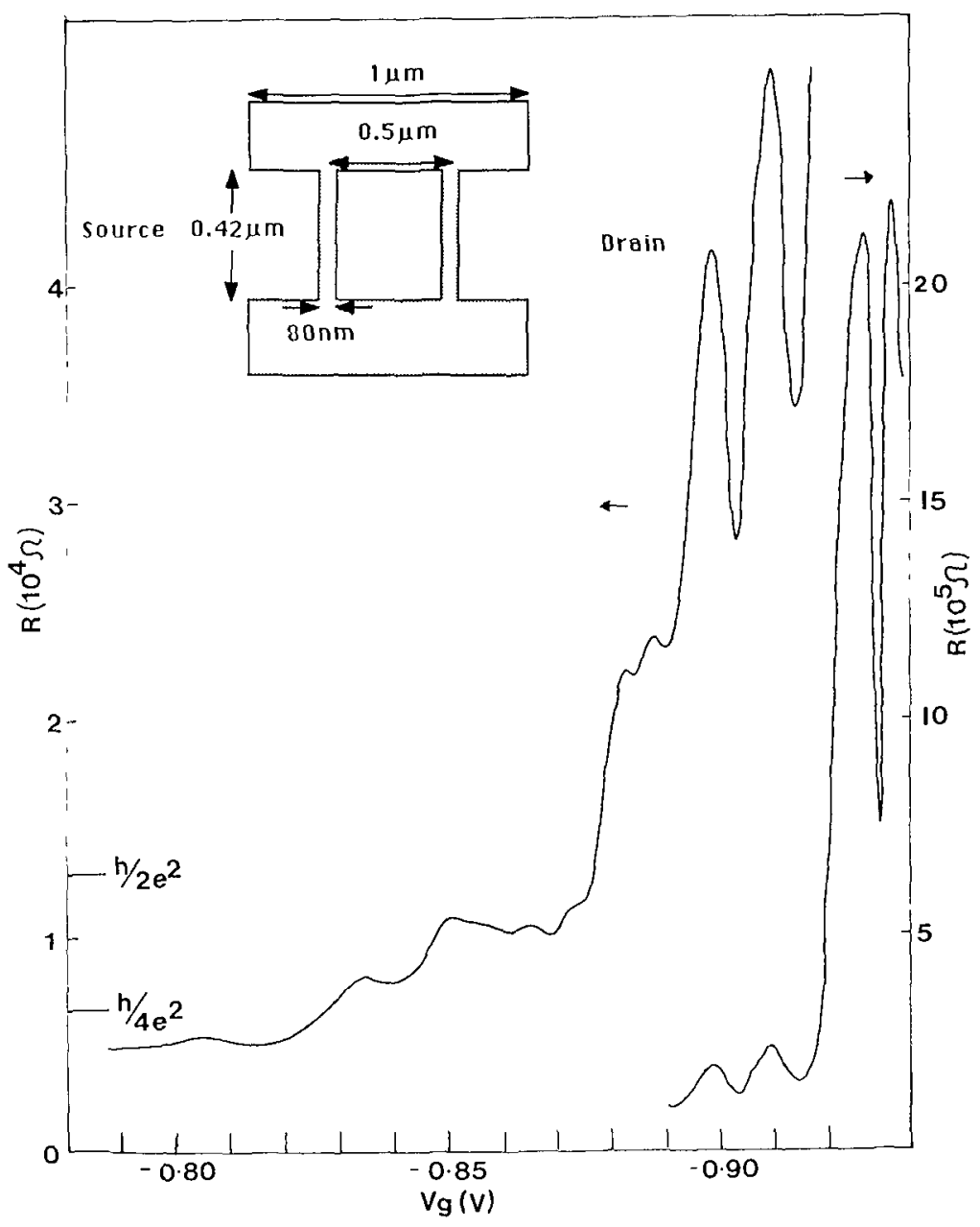

FIG. 70. Resistance versus gate voltage of a cavity (defined by gates on top of a GaAs-AlGaAs heterostructure; see inset), showing plateaulike features (for $R \leqq h / 2 e^{2}$ ) and tunneling resonances (for $R \gtrsim h / 2 e^{2}$ ). The left- and right-hand curves refer to the adjacent resistance scales. Taken from C. E. Smith et al., Surf. Sci. 228, 387 (1990).

the resistance. Very reasonable agreement with the experimental data in the ballistic regime was obtained. The tunneling regime was not compared in detail with the experimental data. The results were found to depend rather critically on the assumed chape of the potential, in particular on the rounding of the tops of the potential barriers. Martin-Moreno and Smith also investigated the effects of asymmetries in the device structure on the tunneling resonances and found in particular that small differences in the two barrier 
heights (of order $10 \%$ ) lead to a sharp suppression of the resonances, a finding that sheds light on the fact that they were observed in certain devices only Experimentally, the effect of a magnetic field on the oscillations in the resistance versus gate voltage was also investıgated ${ }^{399-401}$ A strong suppression of the peaks was found in relatively weak magnetic fields (of about $03 \mathrm{~T})$

Tunneling through a cavity, as in the experiment by Smith et al, ${ }^{399}{ }^{401}$ is formally equivalent to tunneling through an impurity state (see, e g, Refs 402 and 403) The dramatic subthreshold structure found in the conductance of quasi-one-dimensional MOSFETs has been interpreted in terms of resonant tunnelıng through a series of localized states ${ }^{3235-37}$ Kopley et al ${ }^{404}$ have observed large conductance peaks in a MOSFET with a split gate (see Fig 71) Below the 200-nm-wide slot in the gate, the inversion layer is interrupted by a potential barrier Pronounced conductance peaks were seen at $05 \mathrm{~K}$ as the gate voltage was varied in the region close to threshold (see Fig 72) No clear correlation was found between the channel width and the peak spacing or amplitude The peaks were attributed to resonant transmission through single localized states associated with bound states in the $S_{1}$ band gap in the noninverted region under the gate

The theory of resonant tunneling of noninteracting electrons through localized states between two-dimensional reservoirs was developed by Xue and $\mathrm{Lee}^{405}$ (see also Refs 159 and 406) If the resonances are well separated in energy, a single localized state will give the dominant contribution to the transmission probability The maximum conductance on resonance is then $e^{2} / h$ (for one spin direction), regardless of the number of channels $N$ in the reservoirs 405406 This maximum (which may be interpreted as a contact resistance, similar to that of a quantum point contact) is attained if the localized state has identical leak rates $\Gamma_{\mathrm{L}} / \hbar$ and $\Gamma_{\mathrm{R}} / \hbar$ to the left and right reservoirs Provided these leak rates are small (cf Section 21) the conductance $G$ as a function of Fermi energy $E_{\Gamma}$ is a Lorentzian centered around the resonance energy $E_{0}$

$$
G\left(E_{\mathrm{F}}\right)=\frac{e^{2}}{h} \frac{\Gamma_{\mathrm{L}} \Gamma_{\mathrm{R}}}{\left(E_{\mathrm{F}}-E_{0}\right)^{2}+\frac{1}{4}\left(\Gamma_{\mathrm{L}}+\Gamma_{\mathrm{R}}\right)^{2}}
$$

This is the Breit-Wigner formula of nuclear physics ${ }^{93}$ For an asymmetr1cally placed impurity the peak height is reduced below $e^{2} / h$ (by up to a factor $4 \Gamma_{\mathrm{R}} / \Gamma_{\mathrm{L}}$, if $\left.\Gamma_{\mathrm{L}} \gg \Gamma_{\mathrm{R}}\right)$

${ }^{402}$ S J Bending and M R Bedsley, Phys Rev Lett 55, 324 (1985)

${ }^{403}$ A B Fowler, G L T1mp, J J Wainer, and R A Webb, Phys Rev Lett 57, 138 (1986)

${ }^{404}$ T E Kopley, P L McEuen and R G Wheeler, Phys Rev Lett 61, 1654(1988), see also T E Kopley, Ph D thesis, Yale University, 1989

${ }^{405} \mathrm{~W}$ Xue and P A Lee Phys Rev B 38, 3913 (1988)

${ }^{406} \mathrm{~V}$ Kalmeyer and R B Laughlın, Phys Rev B 35, 9805 (1987) 


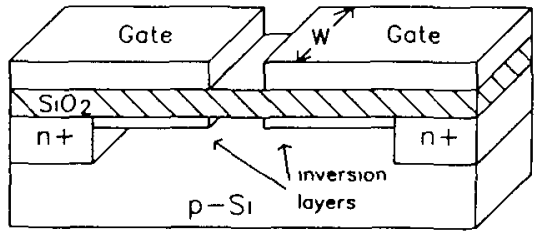

(o)

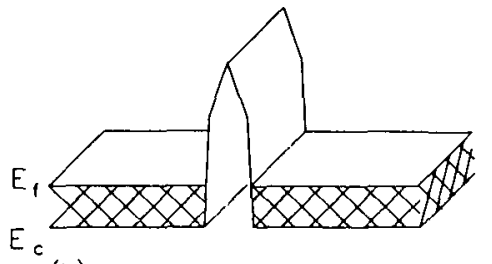

(b)
FIG 71 Schematic diagram of a $\mathrm{S}_{1}$ MOSFET with a split gate (d), which creates d potential barrier in the inversion layer (b) Taken from T E Kopley et al Phys Rev Lett 61, 1654 (1988)

The amplitudes of the peaks observed by Kopley et al ${ }^{404}$ were found to be in agreement with this prediction, while the line shape of an isolated peak could be well described by a Lorentzian (see inset of $F_{1} g$ 72) (Most of the peaks overlapped, hampering a line-shape analysıs) In addition, they studied the effect of a strong magnetic field on the conductance peaks and found that the amplitudes of most peaks were substantially suppressed This was interpreted as a reduction of the leak rates because of a reduced overlap between the wave functions on the impurity and the reservors The amplitude of one particular peak was found to be unaffected by the field, indicative of a symmetrically placed impurity in the barrier $\left(\Gamma_{R}=\Gamma_{L}\right)$, while the width of that peak was reduced, in agreement with Eq (171) This study therefore exhibits many characteristic features of resonant tunneling through a single localized state

Transmission resonances due to an impurity in a quantum point contact

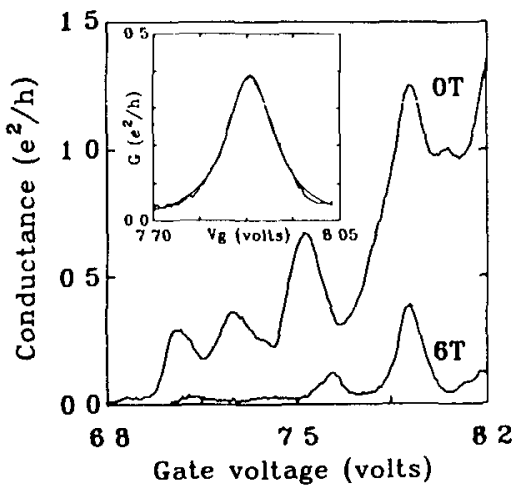

Fig 72 Oscillations in the conductance as d function of gate voltage at $05 \mathrm{~K}$ are attributed to resonant tunneling through localized states in the potential barrier A second trace is shown for a magnetic field of $6 T$ (with a horizontal offset of $-004 \mathrm{~V}$ ) The inset is a close up of the largest peak at $6 T$, together with a Lorentzian fit Taken from T E Kopley et al Phys Rev Lett 61, 1654 (1988) 


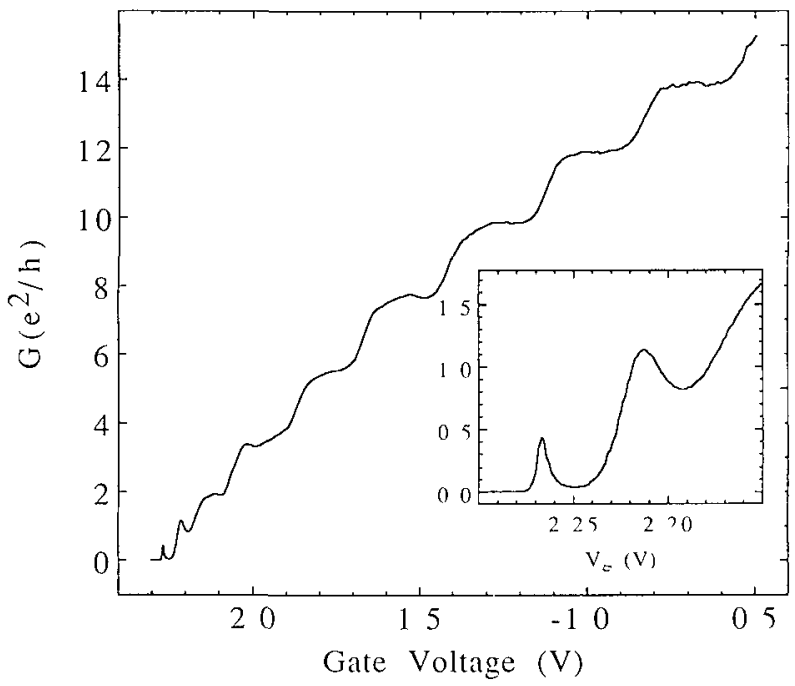

FIG 73 Conductance as a function of gate voltage for a quantum point contact at $055 \mathrm{~K}$ The inset is a close-up of the low-conductance regime, showing peaks attributed to transmission resonances associated with impurity states in the constriction Taken from $\mathrm{P}$ L McEuen et al, Surf Scl 229, 312 (1990)

or narrow channel have been studied theoretically in Refs 241,407 , and 408 In an experiment it may be difficult to distınguish these resonances from those associated with reflection at the entrance and exit of the quantum point contact (discussed in Section 13) A conductance peak associated with resonant tunneling through an impurity state in a quantum point contact was reported by McEuen et al ${ }^{409}$ The experimental results are shown in Fig 73 The resonant tunneling peak is observed near the onset of the first conductance plateau, where $G<2 e^{2} / h$ A second peak seen in Fig 73 was conjectured to be a signature of resonant scattering, in analog with similar processes known in atomic physics 410

We want to conclude this subsection on transmission resonances by discussing an experiment by Smith et al 401411 on what is essentially a Fabry-Perot interferometer The device consists of a point contact with external reflectors in front of its entrance and exit The reflectors are potential barriers erected by means of two additıonal gate electrodes (see Fig 74a) By

${ }^{407}$ C S Chu and R S Sorbello, Phys Rev B 40, 5941 (1989)

${ }^{408} \mathrm{~J}$ Masek, P Lipavsky, and B Kramer, $J$ Phys Condens Matter 1, 6395 (1989)

${ }^{409}$ P L McEuen, B W Alphenaar, R G Wheeler, and R N Sacks, Surf Scl 229, 312 (1990)

${ }^{410} \mathrm{G}$ J Schulz, Rev Mod Phys 45, 378 (1973)

${ }^{411}$ C G Smith, M Pepper, J E Frost, D G Hasko, D C Peacock, D A Ritchie, and G A C Jones, J Phys Condens Matter 1, 9035 (1989) 
(a)

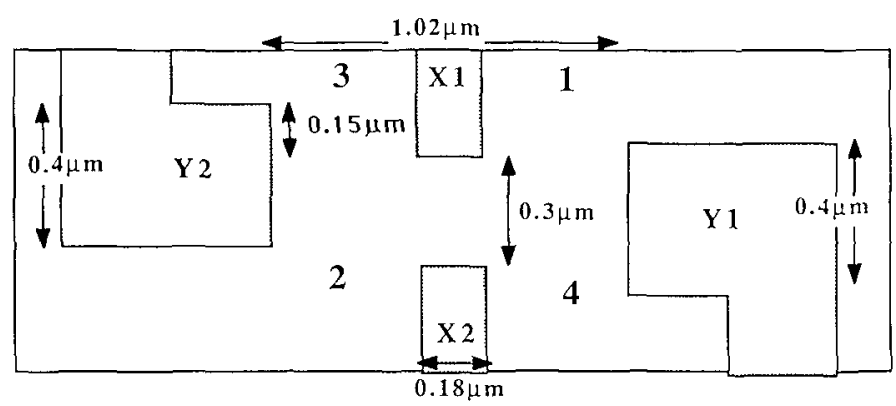

(b)

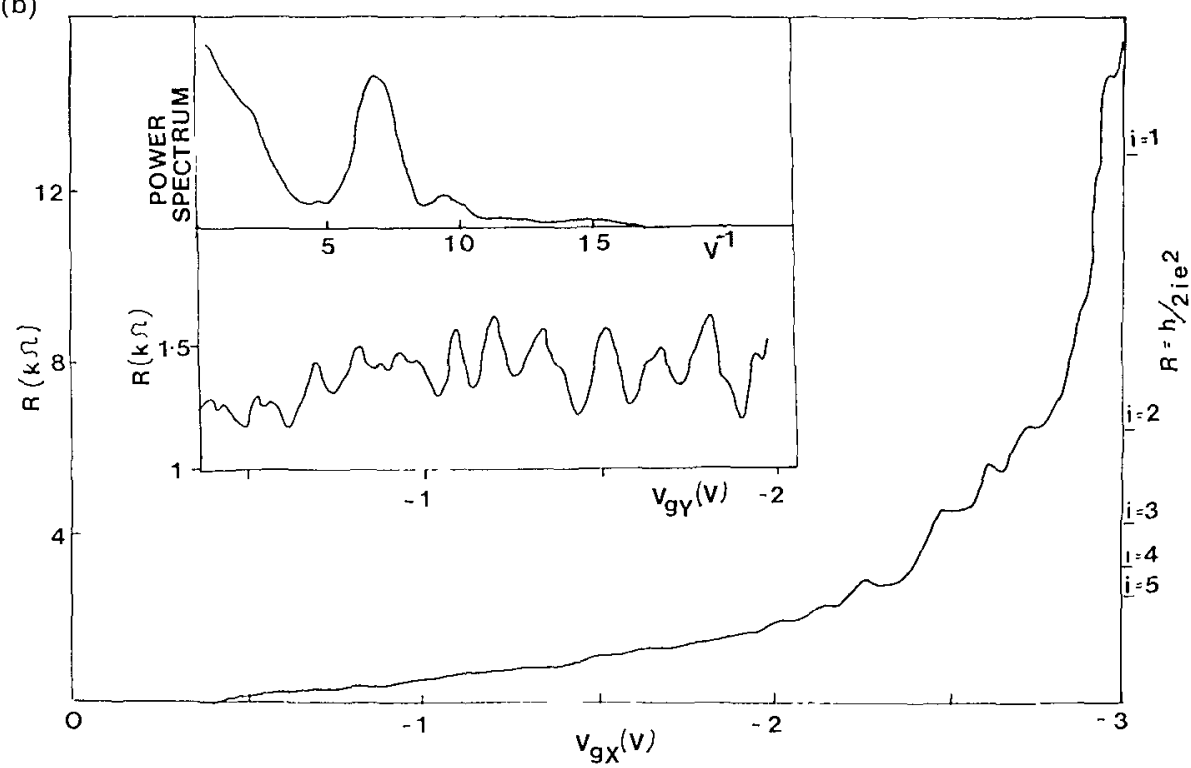

FIG. 74. (a) Schematic diagram of a constriction with two adjustable external reflectors defined by gates on top of a GaAs-AlGaAs heterostructure. (b) Plot of the constriction resistance as a function of gate voltage with the external reflector gates (Y1, Y2) grounded. Inset: Fabry-Perot-type transmission resonances due to a variation of the gate voltage on the reflectors (Y1, Y2) (bottom panel), and Fourier power spectrum (top panel). Taken from C. G. Smith et al., Surf. Sci. 228, 387 (1990).

varying the gate voltage on the external reflectors of this device, Smith et al. could tune the effective cavity length without changing the width of the narrow section. This experiment is therefore more controlled than the quantum dot experiment ${ }^{399-401}$ discussed earlier. The resulting periodic transmission resonances are reproduced in Fig. 74b. A new oscillation appears each time the separation between the reflectors increases by $\lambda_{F} / 2$. A numerical calculation for a similar geometry was performed by Avishai $e t$ 
$a l^{412}$ The significance of this experıment is that it is the first clear realization of an electrostatically tuned electron interferometer Such a device has potential transistor applications Other attempts to fabricate an electrostatic interferometer have been less succesful The electrostatic Aharonov-Bohm effect in a ring was discussed in Section 8 The solid-state analogue of the microwave stub tuner (proposed by Sols et $a l^{413}$ and by Datta ${ }^{414}$ ) was studied experimentally by Miller et al ${ }^{415}$ The idea is to modify the transmission through a narrow channel by changing the length of a side branch (by means of a gate across the side branch) Miller et al have fabricated such a T-shaped conductor and found some evidence for the desired effect Much of the structure was due, however, to disorder-related conductance fluctuations The electrostatic Aharonov-Bohm effect had similar problems Transport in a long and narrow channel is simply not fully ballıstıc, because of partially diffuse boundary scatterıng and impurity scattering The device studied by Smith et al worked because it made use of a very short constriction (a quantum point contact), while the modulation of the interferometer length was done externally in the wide 2DEG, where the effects of disorder are much less severe (in high-mobility material)

\section{b Coulomb Blockade}

In this subsection we would like to speculate on the effects of electronelectron interactions on tunneling through impurities in narrow semiconductor channels, in relation to a recent paper in which Scott-Thomas et al ${ }^{416}$ announced the discovery of conductance oscillations periodic in the density of a narrow $S_{1}$ inversion layer The device features a contınuous gate on top of a split gate, as illustrated schematically in Fig 75 In the experiment, the voltage on the upper gate is varied while the split-gate voltage is kept constant Figure 76 shows the conductance as a function of gate voltage at $04 \mathrm{~K}$, as well as a set of Fourier power spectra obtained for devices of different length A striking pattern of rapid periodic oscillations is seen No correlation is found between the periodicity of the oscllations and the channel length, in contrast to the transmission resonances in ballistic constrictions discussed in Sections 13 and 17 a The oscillations die out as the channel conductance increases toward $e^{2} / h \approx 4 \times 10^{-5} \Omega^{-1}$ The conductance peaks are relatively insensitive to a change in temperature, while the minima depend exponentially on temperature as $\exp \left(-E_{\mathrm{a}} / k_{\mathrm{B}} T\right)$, with an activation energy $E_{\mathrm{a}} \approx 50 \mu \mathrm{eV}$ Pronounced nonlinearities occur in the

${ }^{412}$ Y Avishai, M Kaveh, and Y B Band, preprint

${ }^{413}$ F Sols, M Macucci, U Ravioh, and K Hess, Appl Phys Lett 54, 350 (1989)

${ }^{414} S$ Datta Superlattices and Microstructures 6, 83 (1989)

${ }^{415}$ D S Miller, R K Lake, S Datta, M S Lundstrom, and R Retfenberger, in Ref 15

${ }^{416}$ J H F Scott-Thomas, S B Field, M A Kastner, H I Smith, and D A Antoniadis, Phys

Rev Lett 62, 583 (1989) 
(a)
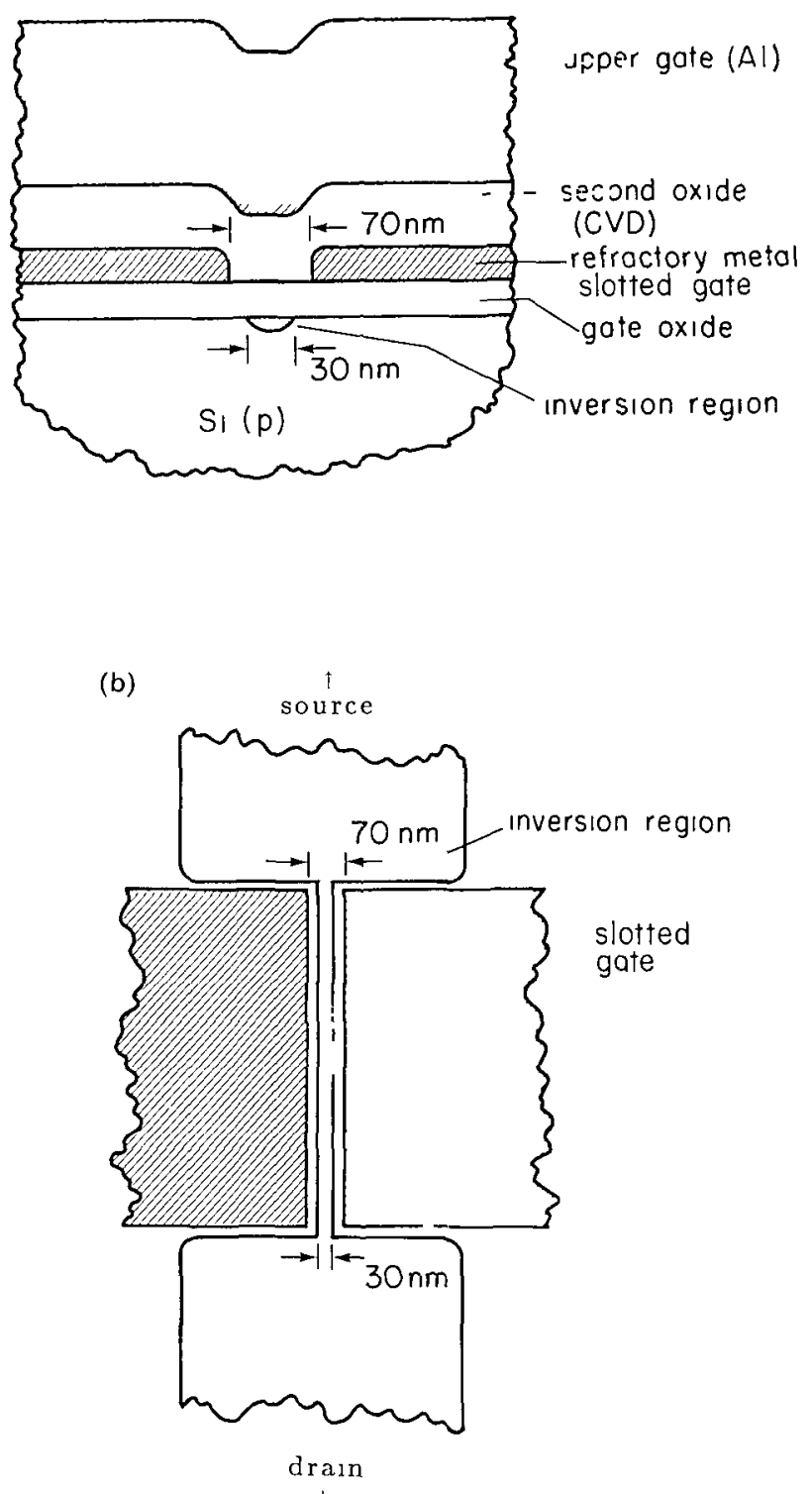

FIG 75 Schematic cross sectional (a) and top (b) view of a double-gate S1 MOSFET device The lower split gate is at a negative voltage, confining the inversion layer (due to the positive voltage on the upper gate) to a narrow channel Taken from J H F Scott-Thomas et al, Phys Rev Lett 62, 583 (1989) 


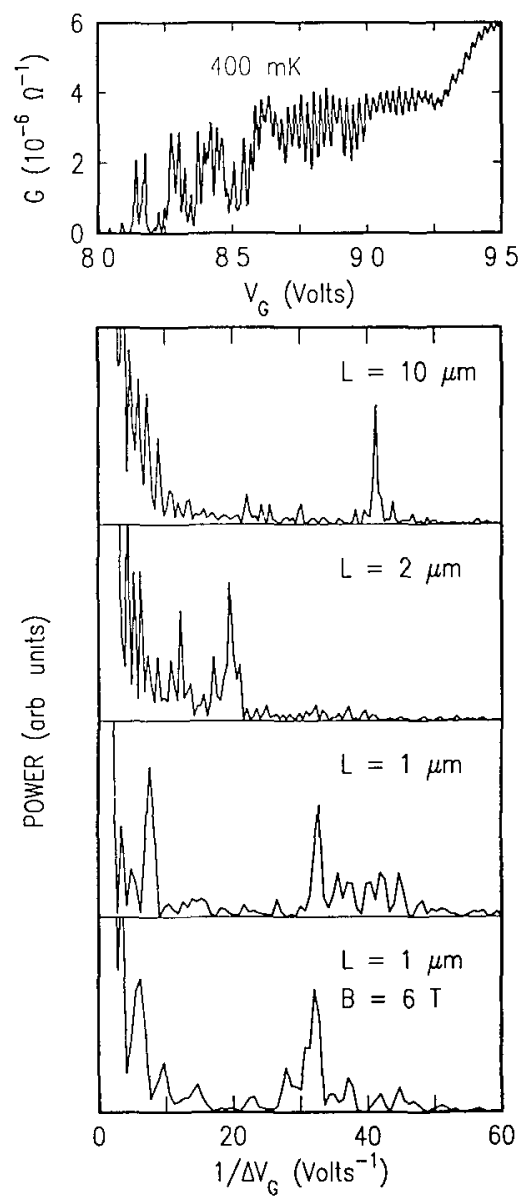

Fig 76 Top panel Periodic oscillatıons in the conductance versus gate voltage at $04 \mathrm{~K}$ for a $10-\mu \mathrm{m}$-long inversion channel Next three panels Fourter power spectra of this curve and of data obtained for 2- and 1- $\mu \mathrm{m}$-long channels Bottom panel Fourier spectrum for the 1- $\mu \mathrm{m}$-long device in a magnetic field of 6 T Taken from J H F Scott-Thomas et al, Phys Rev Lett 62, 583 (1989)

current as a function of source-drain voltage An interpretation in terms of pinned charge density waves was suggested, ${ }^{416}$ based on a model due to Larkin and $\mathrm{Lee}^{417}$ and Lee and Rice ${ }^{418}$ In such a model, one expects the conductance to be thermally activated, because of the pinning of the charge density wave by impurities in the one-dimensional channel The activation

${ }^{417}$ A I Larkin and P A Lee, Phys Rev B 17, 1596 (1978)

${ }^{418} \mathrm{P}$ A Lee and T M Rice, Phys Rev B 19, 3970 (1979) 

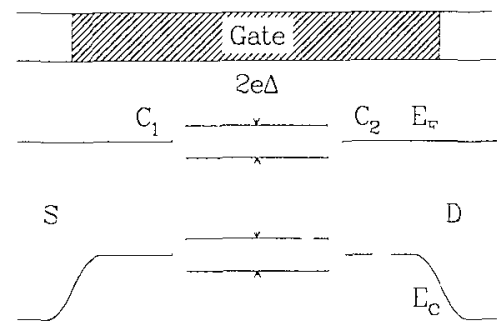

FIG 77 Schematic representation of the bottom of the conduction band $E_{\mathrm{c}}$ and Fermi energy $E_{\mathrm{F}}$ in the device of Fig 76 along the channel The band bending at the connections of the narrow channel to the wide source $S$ and drain $D$ regions arises from the higher threshold for the electrostatic creation of a narrow inversion layer by a gate (shaded part) Tunnel barriers associdted with two scattering centers are shown The maximum Fermı energy difference sus tamable by the Coulomb blockade, $\Delta E_{\mathrm{F}}= \pm e \Delta$ (where $\Delta=e / 2 C$ with $C=C_{1}+C_{2}$ ) is indicated Taken from $\mathrm{H}$ van Houten and $\mathrm{C}$ W J Beenakker Phys Rev Lett 63, 1893 (1989)

energy is determined by the most strongly pinned segment in the channel, and periodic oscillations in the conductance as a function of gate voltage correspond to the condition that an integer number of electrons is contained between the two impurities delimiting that specific segment The same interpretation has been given to a similar effect observed in a narrow channel in a GaAs-AlGaAs heterostructure by Merrav et al ${ }^{85}$

We have proposed ${ }^{419}$ an alternative single-electron explanation of the remarkable effect discovered by Scott-Thomas et al, ${ }^{416}$ based upon the concept of the Coulomb blockade of tunneling mentioned at the beginning of this section Likharev ${ }^{391}$ and Mullen et al ${ }^{420}$ have studied theoretically the possibility of removing the Coulomb blockade by capacitive charging (by means of a gate electrode) of the region between two tunnel barners They found that the conductance of this system exhibits periodic peaks as a function of gate voltage, due to the modulation of the net charge $(\bmod e)$ on the interbarrier region Following the theoretical papers, ${ }^{391420}$ the authors in Ref 419 proposed that the current through the channel in the experiment of Scott-Thomas et al ${ }^{416}$ is limited by tunneling through potential barriers constituted by two dominant scattering centers that delimit a segment of the channel (see Fig 77) Because the number of electrons localized in the region between the two barriers is necessarily an integer, a charge imbalance, and hence an electrostatic potential difference, arises between this region and the adjacent regions connected to wide electron gas reservoirs As the gate voltage is varied, the resulting Fermi level difference $\Delta E_{\mathrm{F}}$ oscillates in a sawtooth pattern between $\pm e \Delta$, where $\Delta=e / 2 C$ and $C=C_{1}+C_{2}$ is the effective capacitance of the region between the two barriers The singleelectron charging energy $e^{2} / 2 C$ maintains the Fermı level difference until $\Delta E_{\mathrm{F}}= \pm e \Delta$ (this is the Coulomb blockade) When $\Delta E_{\mathrm{F}}= \pm e \Delta$, the energy

${ }^{419} \mathrm{H}$ van Houten and C W J Beenakker, Phys Rev Lett 63, 1893 (1989)

${ }^{420} \mathrm{~K}$ Mullen, E Ben-Jacob, R C Jaclevic, and Z Schuss, Phys Rev B 37, 98 (1988), M Amman,

K Mullen, and E Ben-Jacob, $J$ Appl Phys 65, 339 (1989) 
required for the transfer of a single electron to (or from) the region between the two barriers vanishes so that the Coulomb blockade is removed The conductance then shows a maximum at low temperatures $T$ and sourcedrain voltages $V\left(k_{B} T / e, V \lesssim \Delta\right)$ We note that in the case of very different tunnelıng rates through the two barriers, one would expect steps in the current as a function of source-drain voltage, which are not observed in the experiments 85416 For two similar barriers this "Coulomb starcase" is suppressed ${ }^{420}$ The oscillation of the Fermi energy as the gate voltage is varied thus leads to a sequence of conductance peaks The periodicity of the oscillations corresponds to the addition of a single electron to the region between the two scattering centers forming the tunnel barriers, so the oscillations are periodic in the density, as in the experiment This singleelectron tunneling mechanism also explains the observed activation of the conductance minima and the insensitivity to a magnetic field ${ }^{85416}$ The capacitance associated with the region between the scattering centers is hard to ascertain The experimental value of the activation energy $E_{\mathrm{a}} \approx 50 \mu \mathrm{eV}$ would imply $C \approx e^{2} / 2 E_{\mathrm{a}} \approx 10^{-15} \mathrm{~F}$ Kastner et al ${ }^{421}$ argue that the capacitance in the device is smaller than this amount by an order of magnitude (the increase in the effective capacitance due to the presence of the gdte electrodes is taken into account in their estımate) In addition, they point to a discrepancy between the value for the Coulomb blockade inferred from the nonlinear conductance and that from the thermal activation energy The temperature dependence of the oscillatory conductance was found to be qualitatively different in the experiment by Merrav et al ${ }^{85}$ At elevated temperatures an exponential $T$-dependence was found, but at low temperatures the data suggest a much weaker $T$-dependence It is clear that more experimental and theoretical work is needed to arrive at a definitive interpretation of this intriguing phenomenon

It would be of interest to study the effects of the Coulomb blockade of tunneling in a more controlled fashion in a structure with two adjustable potential barriers Such an experiment was proposed by Glazman and Shekter, ${ }^{422}$ who studied theoretically a system similar to the cavity of the experiments by Smith et al ${ }^{399} 401$ (discussed in Section 17 a) A difficulty with this type of device is, as pointed out in Ref 422 , that a variation in gate voltage affects the barrier height (and thus their transparency) as well as the charge in the cavity This is expected to lead to an exponential damping of the oscillations due to the Coulomb blockade ${ }^{391420}$ A characteristic feature of these oscillations is their insensitivity to an applied magnetic field, which can serve to distinguish the effect from oscillations due to resonant tunneling

\footnotetext{
${ }^{421}$ M A Kastner, S B Field, U Merrav, J H F Scott-Thomas, D A Antoniddis, and M I

Smith, Phys Rev Lett 63, 1894 (1989)

${ }^{422}$ L I Gldzman and R I Shekhter, J Phys Condens Matter 1, 5811 (1989)
} 
(Section 17 a) The field dependence of the peaks observed by Smith et $a l^{399} 401$ in the tunneling regime was not reported, so the question of whether or not the Coulomb oscillations are observed in their experiment remains unanswered In our opinıon, substantial progress could be made with the development of thin tunnel barriers of larger height, which would be less sensitive to the application of an external gate voltage If our interpretatıon of the experiments by Scott-Thomas et al ${ }^{416}$ and Merrav et al ${ }^{85}$ is correct, such tunneling barriers might be formed by the incorporation of negatıvely charged impurities (e g, 1onized acceptors) in a narrow electron gas channel This speculation is based on the fact that such acceptor impurities are present in the $S_{1}$ inversion layers of the experiment of ScottThomas et al, ${ }^{416}$ as well as in the $p-n$ junctions employed for lateral confinement by Merrav et al ${ }^{85}$

As we were completing this review, we learned of several experiments that demonstrate the Coulomb blockade in split-gate confined GaAs-AlGaAs heterostructures ${ }^{423-425}$ These experiments should open the way for the controlled study of the effects of Coulomb interactions on tunneling in semiconductor nanostructures

\section{Adiabatic Transport}

\section{Edge Channels and the Quantum Hall Effect}

In this section we give an overview of the characteristics of adiabatic transport via edge channels in the regime of the quantum Hall effect as a background to the following sections We restrict ourselves here to the integer quantum Hall effect, where the edge channels can be described by singleelectron states Recent developments on adiabatic transport in the regime of the fractional quantum Hall effect (which is fundamentally a many-body effect) will be considered in Section 20

\section{a Introduction}

Both the quantum Hall effect (QHE) and the quantized conductance of a ballistic point contact are described by the same relation, $G=N e^{2} / h$, between the conductance $G$ and the number $N$ of propagatıng modes at the Fermi level (counting both spin directions separately) The smooth transition from zero-field quantization to QHE that follows from this relation is evident from Fig 48 The nature of the modes is very different, however, in weak and strong magnetic fields As we discussed in Section $12 \mathrm{a}$, the propagating

${ }^{423}$ R J Brown, M Pepper, H Ahmed, D G Hasko, R A Ritchie, J E F Frost, D C Peacock, and G A C Jones, $J$ Phys Condens Matter, 2, 2105 (1990)

${ }^{424} \mathrm{~L} P$ Kouwenhoven, private communication, $\mathrm{R}$ Haug, private communication

${ }^{425}$ U Meirdv, M A Kastner, and S J Wind, Phys Rev Lett 65, 771 (1990) 
modes in a strong magnetic field consist of edge states, which interact with one of the sample edges only Edge states with the same mode index are referred to collectively as an edge channel Edge channels at opposite edges propagate in opposite directions In a weak magnetic field, in contrast, the modes consist of magnetoelectric subbands that interact with both edges In that case there is no spatial separation of modes propagating in opposite directions

The different spatial extension of edge channels and magnetoelectric subbands leads to an entirely different sensitivity to scattering processes in weak and strong magnetic fields Firstly, the zero-field conductance quantization is destroyed by a small amount of elastic scattering (due to impurities or roughness of the channel boundaries, of Refs 313,316, 317, 407, and 408), while the QHE is robust to scattering ${ }^{97}$ This difference is a consequence of the suppression of backscattering by a magnetic field discussed in Section $13 \mathrm{~b}$, which itself follows from the spatial separation at opposite edges of edge channels moving in opposite directions Second, the spatial separation of edge channels at the same edge in the case of a smooth confining potential opens up the possibility of adiabatic transport $(1 \mathrm{e}$, the full suppression of interedge channel scattering) In weak magnetic fields, adiabaticity is of importance within a point contact, but not on longer length scales (cf Sections $13 \mathrm{a}$ and $15 \mathrm{a}$ ) In a wide 2DEG region, scattering among the modes in weak fields establishes local equilibrium on a length scale given by the inelastic scattering length (which in a high-mobility GaAs-AlGaAs heterostructure is presumably not much longer than the elastic scattering length $l \sim 10 \mu \mathrm{m})$ The situation is strikingly different in a strong magnetic field, where the selective population and detection of edge channels observed by van Wees et al ${ }^{426}$ has demonstrated the persistence of adiabaticity outside the point contact

In the absence of interedge channel scattering the various edge channels at the same boundary can be occupied up to different energies and consequently carry different amounts of current The electron gas at the edge of the sample is then not in local equilibrium Over some long distance (which is not yet known precisely) adiabatıcity breaks down, leading to a partial equilibration of the edge channels However, as demonstrated by Komiyama et al ${ }^{427}$ ard by others, ${ }^{307428-430}$ local equilibrium is not fully established even on

\footnotetext{
${ }^{426}$ B J van Wees, E M M Willems, C J P M Harmans, C W J Beenakker, H van Houten, J G Williamson, C T Foxon, and J J Harris, Phys Rev Lett 62, 1181 (1989)

${ }^{427} \mathrm{~S}$ Komiyama, H Hira1, S Sasa, and S Hiyamizu, Phys Rev B 40, 12566 (1989)

${ }^{428} B$ J van Wees, E M M Willems, L $P$ Kouwenhoven, C J P M Harmans, J G Willamson, C T Foxon, and J J Harris, Phys Rev B 39, 8066 (1989)

${ }^{429}$ B W Alphenaar, P L McEuen, R G Wheeler, and R N Sacks, Phys Rev Lett 64, 677 (1990)

${ }^{430}$ R J Haug and K von Klitzıng, Europhys Lett 10, 489 (1989)
} 
macroscopic length scales exceeding $025 \mathrm{~mm}$ Since local equilibrium is a prerequisite for the use of a local resistivity tensor, these findings imply a nonlocality of the transport that had not been anticipated in theories of the QHE (which are commonly expressed in terms of a local resistivity) ${ }^{97}$

A theory of the QHE that is able to explain anomalies resultıng from the absence of local equilibrium has to take into account the properties of the current and voltage contacts used to measure the Hall resistance That is not necessary if local equilibrium is established at the voltage contacts, for the fundamental reason that two systems in equilbrium that are in contact have identical electrochemical potentials In the Landauer-Buttıker formalısm described in Section $12 \mathrm{~b}$, the contacts are modeled by electron gas reservorrs and the resistances are expressed in terms of transmission probabilities of propagating modes at the Fermi level from one reservoir to the other This formalısm is not restrıcted to zero or weak magnetıc fields, but can equally well be applied to the QHE, where edge channels form the modes In this way Buttiker could show ${ }^{12}$ that the nonideality of the coupling of the reservorrs to the conductor affects the accuracy of the QHE in the absence of local equilibrium An ideal contact in the QHE is one that establishes an equilibrium population among the outgoing edge channels by distributing the injected current equally among these propagating modes (this is the equipartitioning of current discussed for an ideal electron waveguide in Section 12 b) A quantum point contact that selectively populates certain edge channels ${ }^{426}$ can thus be seen as an extreme example of a nonideal, or disor dered, contact

\section{b Edge Channels in a Disordered Conductor}

After this general introduction, let us now discuss in some detail how edge channels are formed at the boundary of a $2 \mathrm{DEG}$ in a strong magnetic field In Section $12 \mathrm{a}$ we discussed the edge states in the case of a narrow channel without disorder, relevant for the point contact geometry Edge states were seen to originate from Landau levels, which in the bulk lie below the Fermı lcvel but rise in energy on approaching the sample boundary (cf Fig 40b) The point of intersection of the $n$th Landau level $(n=1,2, \quad)$ with the Ferm level forms the site of edge states belonging to the $n$th edge channel The number $N$ of edge channels at $E_{\mathrm{F}}$ is equal to the number of bulk Landau levels below $E_{\mathrm{r}}$ This description can easily be generalized to the case of a slowly varying potential energy landscape $V(x, y)$ in the $2 \mathrm{DEG}$, in which case a semiclassical analysis can be applied ${ }^{431}$ The energy $E_{\mathrm{F}}$ of an electron at the Fermı level in a strong magnetic field contains a part $\left(n-\frac{1}{2}\right) \hbar \omega_{\mathrm{c}}$ due to the

${ }^{431}$ R Kubo, S J Miyake, and N Hashitsume, "Sold State Physics," Vol 17 (F Settz and D Turnbull, eds) Academic Press, New York, 1965 M Tsukada, J Phys Soc Jap, 41, 1466 (1976) 
quantized cyclotron motion and a part $\pm \frac{1}{2} g \mu_{\mathrm{B}} B$ (depending on the spin direction) from spin splitting The remainder is the energy $E_{G}$ due to the electrostatic potential

$$
E_{\mathrm{G}}=E_{\mathrm{F}}-\left(n-\frac{1}{2}\right) \hbar \omega_{\mathrm{c}} \pm \frac{1}{2} g \mu_{\mathrm{B}} B
$$

The cyclotron orbit center $\mathbf{R}$ is guided along equipotentials of $V$ at the guiding center energy $E_{\mathrm{G}}$ As derived in Section $11 \mathrm{~b}$, the drift velocity $\mathbf{v}_{\mathrm{drift}}$ of the orbit center (known as the guiding center drift or $\mathbf{E} \times \mathbf{B}$ drift) is given by

$$
\mathbf{v}_{\mathrm{drift}}(\mathbf{R})=\frac{1}{e B^{2}} \nabla V(\mathbf{R}) \times \mathbf{B},
$$

which indeed is parallel to the equipotentials An important distinction with the weak-field case of Section $11 \mathrm{~b}$ is that the spatial extension of the cyclotron orbit can now be neglected, so $V$ is evaluated at the position of the orbit center in Eq (18 2) [compared with Eq (11 1)] The guiding center drift contributes a kinetic energy $\frac{1}{2} m v_{\mathrm{drift}}^{2}$ to the energy of the electron, which is small for large $B$ and smooth $V$ (More precisely, $\frac{1}{2} m v_{\text {drift }}^{2} \ll \hbar \omega_{\mathrm{c}}$ if $|\nabla V| \ll \hbar \omega_{\mathrm{c}} / l_{\mathrm{m}}$, with $l_{\mathrm{m}}$ the magnetic length defined as $\left.l_{\mathrm{m}} \equiv(\hbar / e B)^{1 / 2}\right)$ This kinetic energy term has therefore not been included in Eq (18 1)

The simplicity of the guiding center drift along equipotentials has been originally used in the percolation theory ${ }^{432}{ }^{434}$ of the QHE, soon after its experımental discovery ${ }^{8}$ In this theory the existence of edge states is ignored, so the Hall resistance is not expressed in terms of equilibrium properties of the 2DEG (in contrast to the edge channel formulation that will be discussed) The physical requirements on the smoothness of the disorder potential have received considerable attention ${ }^{435} 436$ in the context of the percolation theory and, more recently, ${ }^{437} 439$ in the context of adiabatic transport in edge channels Strong potential variations should occur on a spatial scale that is large compared with the magnetic length $l_{\mathrm{m}}\left(l_{\mathrm{m}}\right.$ corresponds to the cyclotron radius in the QHE, $l_{\text {cycl }} \equiv l_{\mathrm{m}}(2 n-1)^{1 / 2} \approx l_{\mathrm{m}}$ if the Landau level index $n \approx 1$ ) More rapid potential fluctuations may be present provided their amplitude is much less than $\hbar \omega_{c}$ (the energy separation of Landau levels)

${ }^{432}$ R F Kazarinov and S Luryı, Phys Rev B 25, 7626 (1982), S Luryı and R F Kazarnov, Phys Rev B 27, 1386 (1983), S Lury1, in "High Magnetic Fields in Semiconductor Physics" (G Landwehr, ed) Springer, Berlın, 1987

${ }^{433}$ S V Iordansky, Sold State Comm 43, 1 (1982)

${ }^{434}$ S A Trugman, Phys Rev B 27, 7539 (1983)

${ }^{435}$ R Joynt and R E Prange, Phys Rev B 29, 3303 (1984)

${ }^{436} \mathrm{R}$ E Prange, in Ref 97

${ }^{437} \mathrm{~L}$ I Glazman and M Jonson, $J$ Phys Condens Matter 1, 5547 (1989)

${ }^{438}$ L I Glazman and M Jonson, Phys Rev B 41, 10686 (1990)

${ }^{439} \mathrm{~T}$ Martin and S Feng, Phys Rev Lett 64, 1971 (1990) 


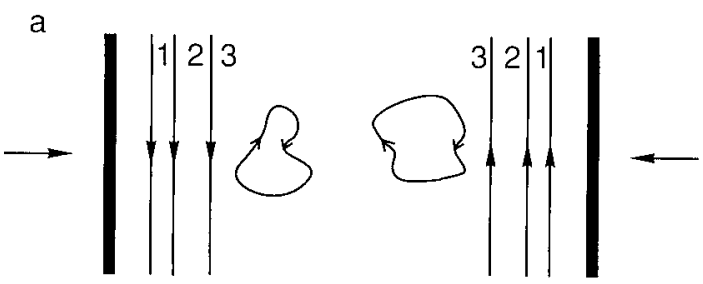

b

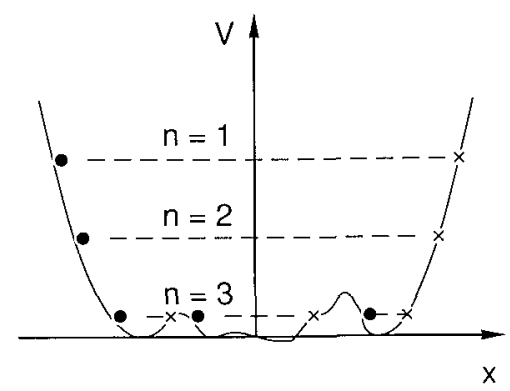

a

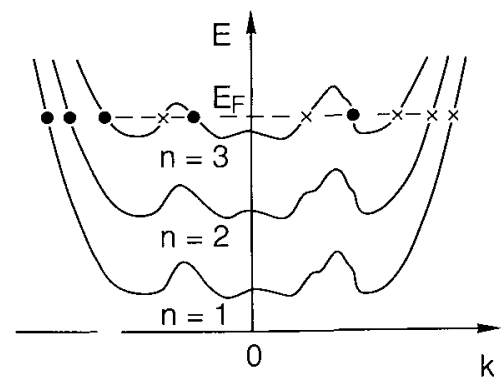

FIG 78 Formation of edge channels in a disordered potential, from various viewpoints discussed in the text

In Fig 78 we have illustrated the formation of edge channels in a smooth potential energy landscape from various viewpoints The wave functions of states at the Fermı level are extended along equipotentials at the guiding center energy (18 1), as shown in Fig 78a (for Landau level index $n=1,2,3$ and a single spin direction) One can distinguish between extended states near the sample boundaries and localized states encircling potential maxima and minıma in the bulk The extended states at the Fermı level form the edge channels The edge channel with the smallest index $n$ is closest to the sample boundary, because it has the largest $E_{\mathrm{G}}[\mathrm{Eq}(181)]$ This is seen more clearly in the cross-sectional plot of $V(x, y)$ in Fig $78 \mathrm{~b}$ (along the line connecting the two arrows in Fig 78a) The location of the states at the Fermi level is 


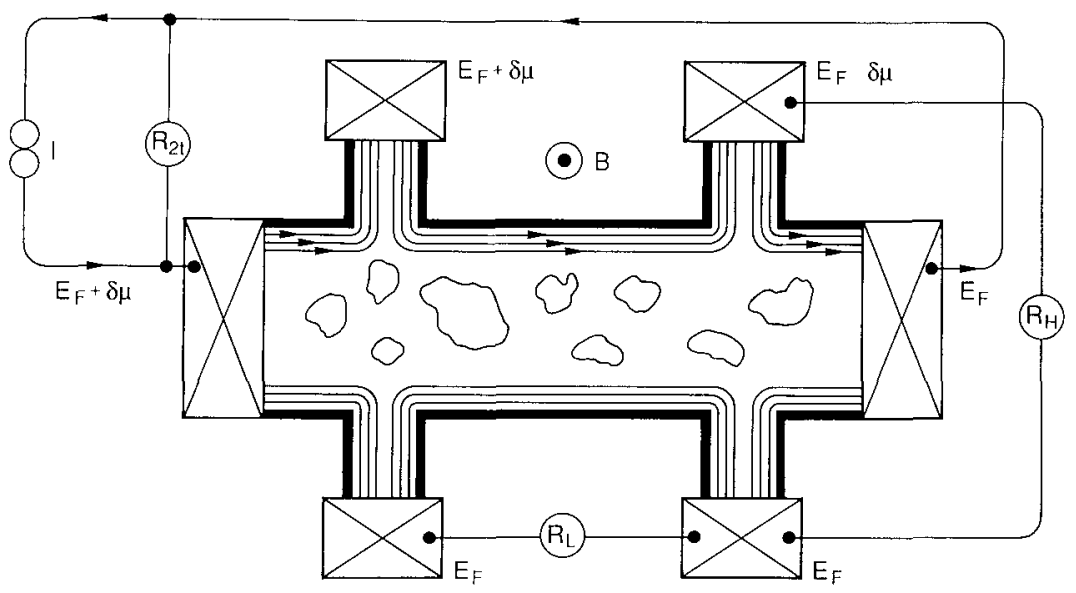

FIG 79 Measurement configuration for the two-terminal resistance $R_{21}$, the four-terminal Hall resistance $R_{\mathrm{H}}$, and the longitudinal resistance $R_{\mathrm{L}}$. The edge channels at the Fermi level are indicated, arrows point in the direction of motion of edge channels filled by the source contact at chemical potential $E_{\mathbf{F}}+\delta \mu$ The current is equipartitioned among the edge channels at the upper edge, corresponding to the case of local equilibrium

indicated by dots and crosses (depending on the direction of motion) The value of $E_{\mathrm{G}}$ for each $n$ is indicated by the dashed line If the peaks and dips of the potential in the bulk have amplitudes below $\hbar \omega_{\mathrm{c}} / 2$, then only states with highest Landau level index can exist in the bulk at the Fermi level This is obvious from $\mathrm{Fig} 78 \mathrm{c}$, which shows the total energy of a state $E_{\mathrm{G}}+\left(n-\frac{1}{2}\right) \hbar \omega_{\mathrm{c}}$ along the same cross section as Fig $78 \mathrm{~b}$ If one identıfies $k=-x e B / \hbar$, this plot can be compared with Fig $40 \mathrm{~b}$ of the dispersion relation $E_{n}(k)$ for a disorder-free electron waveguide in strong magnetic field

A description of the QHE based on extended edge states and localized bulk states, as in Fig 78, was first put forward by Halperin ${ }^{440}$ and further developed by several authors ${ }^{441}{ }^{444}$ In these papers a local equilibrium is assumed at each edge In the presence of a chemical potential difference $\delta \mu$ between the edges, each edge channel carries a current $(e / h) \delta \mu$ and thus contributes $e^{2} / h$ to the Hall conductance (cf the derivation of Landauer's formula in Section 12 b) In this case of local equilibrium the two-terminal resistance $R_{21}$ of the Hall bar is the same as the four-terminal Hall resistance $R_{\mathrm{H}}=R_{2 \mathrm{t}}=h / e^{2} N$ (see Fig 79) The longitudinal resistance vanishes, $R_{\mathrm{L}}=0$

\footnotetext{
${ }^{440}$ B I Halperin, Phys Rev B 25, 2185 (1982)

${ }^{441}$ A H MacDonald and P Streda, Phys Rev B 29, 1616 (1984)

${ }^{442}$ S M Apenko and Yu E Lozovik, J Phys C 18, 1197 (1985)

${ }^{443} \mathrm{P}$ Streda, J Kucera, and A H MacDonald, Phys Rev Lett 59, 1973 (1987)

${ }^{444} \mathrm{~J}$ K Jain and S A Kivelson, Phys Rev B 37, 4276 (1988)
} 
The distinction between a longitudinal and Hall resistance is topological A four-terminal resistance measurement gives $R_{\mathrm{H}}$ if current and voltage contacts alternate along the boundary of the conductor, and $R_{\mathrm{L}}$ if that is not the case There is no need to further characterize the contacts in the case of local equilibrium at the edge

If the edges are not in local equilibrium, the measured resistance depends on the properties of the contacts Consider, for example, a situation in which the edge channels at the lower edge are in equilibrium at chemical potential $E_{\mathrm{F}}$, while the edge channels at the upper edge are not in local equilibrium The current at the upper edge is then not equipartitioned among the $N$ modes Let $f_{n}$ be the fraction of the total current $I$ that is carried by states above $E_{\mathrm{F}}$ in the $n$th edge channel at the upper edge, $I_{n}=f_{n} I$ The voltage contact at the lower edge measures a chemical potential $E_{\mathrm{F}}$ regardless of its properties The voltage contact at the upper edge, however, will measure a chemical potential that depends on how it couples to each of the edge channels The transmission probability $T_{n}$ is the fraction of $I_{n}$ that is transmitted through the voltage probe to a reservoir at chemical potential $E_{\mathrm{F}}+\delta \mu$ The incoming current

$$
I_{1 \mathrm{n}}=\sum_{n=1}^{N} T_{n} f_{n} I, \quad \text { with } \sum_{n=1}^{N} f_{n}=1,
$$

has to be balanced by an outgoing current

$$
I_{\text {out }}=\frac{e}{h} \delta \mu(N-R)=\frac{e}{h} \delta \mu \sum_{n=1}^{N} T_{n}
$$

of equal magnitude, so that the voltage probe draws no net current (In Eq (184) we have applied Eq (1214) to identify the total transmission probability $N-R$ of outgoing edge channels with the sum of transmission probabilities $T_{n}$ of incoming edge channels) The requirement $I_{\mathrm{n}}=I_{\text {out }}$ determines $\delta \mu$ and hence the Hall resistance $R_{\mathrm{H}}=\delta \mu / \mathrm{eI}$

$$
R_{\mathrm{H}}=\frac{h}{e^{2}}\left(\sum_{n-1}^{N} T_{n} f_{n}\right)\left(\sum_{n-1}^{N} T_{n}\right)^{-1}
$$

The Hall resistance has its regular quantized value $R_{\mathrm{H}}=h / e^{2} N$ only if etther $f_{n}=1 / N$ or $T_{n}=1$, for $n=1,2, \quad, N$ The first case corresponds to local equilibrium (the current is equipartitioned among the modes), the second case to an ideal contact (all edge channels are fully transmitted) The LandauerButtıker formalism discussed in Section $12 \mathrm{~b}$ forms the basis on which anomalies in the QHE due to the absence of local equilibrium in combination with nonideal contacts can be treated theoretically ${ }^{112}$

A nonequilibrium population of the edge channels is generally the result of selective backscattering Because edge channels at opposite edges of the 
sample move in opposite directions, backscattering requires scattering from one edge to the other. Selective backscattering of edge channels with $n \geqslant n_{0}$ is induced by a potential barrier across the sample, ${ }^{113,339,340,427}$ if its height is between the guiding center energies of edge channel $n_{0}$ and $n_{0}-1$ (note that the edge channel with a larger index $n$ has a smaller value of $E_{\mathrm{G}}$ ). The anomalous Shubnikov-De Haas effect, ${ }^{428}$ to be discussed in Section 19, has demonstrated that selective backscattering can also occur naturally in the absence of an imposed potential barrier. The edge channel with the highest index $n=N$ is selectively backscattered when the Fermi level approaches the energy $\left(N-\frac{1}{2}\right) \hbar \omega_{\mathrm{c}}$ of the $N$ th bulk Landau level. The guiding center energy of the $N$ th edge channel then approaches zero, and backscattering either by tunneling or by thermally activated processes becomes effective, but for that edge channel only, which remains almost completely decoupled from the other $N-1$ edge channels over distances as large as $250 \mu \mathrm{m}$ (although on that length scale the edge channels with $n \leqslant N-1$ have equilibrated to a large extent). ${ }^{429}$

\section{c. Current Distribution}

The edge channel theory has been criticized on the grounds that experiments measure a nonzero current in the bulk of a Hall bar. ${ }^{445}$ In this subsection we want to point out that a measurement of the current distribution cannot be used to prove or disprove the edge channel formulation of the QHE.

The fact that the Hall resistance can be expressed in terms of the transmission probabilities of edge states at the Fermi level does not imply that these few states carry a macroscopic current, nor does it imply that the current flows at the edges. A determination of the spatial current distribution $i(\mathbf{r})$, rather than just the total current $I$, requires consideration of all the states below the Fermi level, which acquire a net drift velocity because of the Hall field. As we discussed in Section 12.b, knowledge of $i(\mathbf{r})$ is not necessary to know the resistances in the regime of linear response, because the Einstein relation allows one to obtain the resistance from the diffusion constant. Edge channels tell you where the current flows if the electrochemical potential difference $\delta \mu$ is entirely due to a density difference, relevant for the diffusion problem. Edge channels have nothing to say about where the current flows if $\delta \mu$ is mainly of electrostatic origin, relevant for the problem of electrical conduction. The ratio $\delta \mu / I$ is the same for both problems, but $i(\mathbf{r})$ is not.

With this in mind, it remains an interesting problem to find out just how the current is distributed in a Hall bar, or, alternatively, what is the electrostatic potential profile. This problem has been treated theoretically in

${ }^{445}$ M. E. Cage, in Ref. 97. 
many papers ${ }^{446-455}$ In the case of a $3 \mathrm{D}$ conductor, a linearly varying potential and uniform current density are produced by a surface charge As noted by MacDonald et al,${ }^{446}$ the electrostatics is qualitatively different in the $2 \mathrm{D}$ case because an edge charge $\delta(x-W / 2)$ produces a potential proportional to $\ln |x-W / 2|$, which is weighted toward the edge, and hence a concentration of current at the edge

Experiments aımed at measuring the electrostatic potential distribution were originally carried out by attaching contacts to the interior of the Hall bar and measuring the voltage differences between adjacent contacts ${ }^{456-460}$ It was learned from these studies that relatively small inhomogeneities in the density of the 2DEG have a large effect on these voltage differences in the QHE regime The main difficulty in the interpretation of such experiments is that the voltage difference measured between two contacts is the difference in electrochemical potential, not the line integral of the electric field Buttıker ${ }^{461}$ has argued that the voltage measured at an interior contact can exhibit large variations for a small increase in magnetic field without an appreciable change in the current distribution Contactless measurements of the QHE from the absorption of microwave radiation ${ }^{462}$ are one alternative to interior contacts, which might be used to determine the potential (or current) distribution

Fonten et $a l^{463}$ have used the birefringence of GaAs induced by an

${ }^{446}$ A H MacDonald, T M Rice, and W F Brinkman, Phys Rev B 28, 3648 (1983)

${ }^{447} \mathrm{O}$ Heinonen and P L Taylor, Phys Rev B 32, 633 (1985)

${ }^{448} \mathrm{D}$ J Thouless, $J$ Phys $C$ 18, 6211 (1985)

${ }^{449} \mathrm{~V}$ M Pudalov and S G Semenchınsku, Pts'ma Zh Eksp Teor Ftz 42, 188 (1985) [JETP Lett 42, 232 (1985)]

${ }^{450} \mathrm{~W}$ Maass, Europhys Lett 2, $39(1986)$

${ }^{451} \mathrm{Y}$ Ono and T Ohtsukı, $Z$ Phys B 68, 445 (1987), T Ohtsukı and Y Ono, $J$ Phys Soc Jap 58, 2482 (1989)

${ }^{452} \mathrm{R}$ Johnston and L Schwertzer, $Z$ Phys B 70, 25 (1988)

${ }^{453} \mathrm{~V}$ Gudmundsson, R R Gerhardts, R Johnston, and L Schwertzer, $Z$ Phys B 70, 453 (1988)

${ }^{454} \mathrm{~T}$ Ando, $J$ Phys Soc Jap 58, 3711 (1989)

${ }^{455} \mathrm{P}$ C van Son, G H Kruithof, and T M Klapwijk, Surf Scl 229, 57 (1990), P C van Son and

T M Klapwijk, Eur Phys Lett 12, 429 (1990)

${ }^{456} \mathrm{G}$ Ebert, $\mathrm{K}$ von Klitzing, and $\mathrm{G}$ Weimann, $J$ Phys $C$ 18, L257 (1985)

${ }^{457} \mathrm{H}$ Z Zheng, D C Tsur, and A M Chang, Phys Rev B 32, 5506 (1985)

${ }^{458}$ E K Sichel, H H Sample, and J P Salerno, Phys Rev B 32, 6975(1987), E K Sichel, M L

Knowles, and H H Sample, $J$ Phys C 19, 5695 (1986)

${ }^{459} \mathrm{R}$ Woltjer, R Eppenga, J Mooren, C E Timmering, and J P Andre, Europhys Lett 2, 149 (1986)

${ }^{460} \mathrm{~B}$ E Kane, D C Tsul, and G Weimann, Phys Rev Lett 59, 1353 (1987)

${ }^{461} \mathrm{M}$ Buttıker, in Ref 15

${ }^{462} \mathrm{~F}$ Kuchar, Festkorperprobleme 28, 45 (1988)

${ }^{463}$ P F Fontein, J A Kleınen, $\mathrm{P}$ Hendrıks, F A P Blom, J H Wolter, $\mathrm{H}$ G M Locks, F A J

M Driessen, L J Giling, and C W J Beenakker, submitted to Phys Rev B 


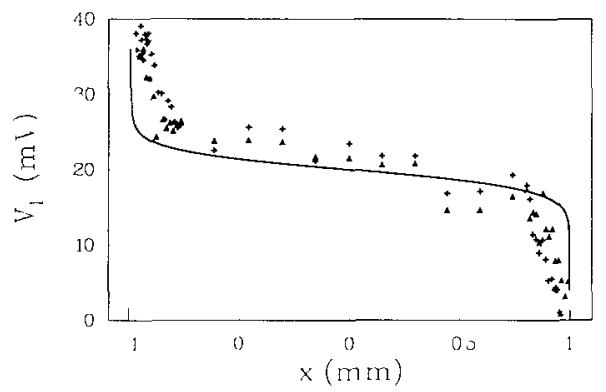

Fig 80 Electrostatic potential $V_{\text {II }}$ induced by passing a current through a Hall bar The sample edges are at $x= \pm 1 \mathrm{~mm}$ The data points are from the experiment of Fontein et al ${ }^{463}$, at two magnetic field values on the $R_{\mathrm{H}}=h / 4 e^{2}$ quantized Hall plateau (triangles $B=5 \mathrm{~T}$, crosses $B=525 \mathrm{~T}$ ) The sold curve is calculated from Eq (189), assuming an impurity-free Hall bar with four filled Landau levels The theory contans no adjustable parameters

electric field to perform a contactless measurement of the electrostatic potential distribution in a Hall bar They measure the Hall potential profile $V_{\mathrm{H}}(x)$ as a change in the local electrostatic potential if a current is passed through the Hall bar The data points shown in Fig 80 were taken at $15 \mathrm{~K}$ for two magnetic field values on the plateau of quantized Hall resistance at $\frac{1}{4} h / e^{2}$ The potential varies steeply at the edges (at $x= \pm 1 \mathrm{~mm}$ in Fig 80) and is approximately linear in the bulk The spatial resolution of the experiment was $70 \mu \mathrm{m}$, limited by the laser beam used to measure the birefringence The current distribution is not directly measured, but can be estimated from the guiding center drift (182) (this assumes a slowly varying potential) The nonequilibrium current density $\imath(x)$ along the Hall bar is then given by

$$
\imath(x)=\frac{e n_{\mathrm{s}}}{B} \frac{d V_{\mathrm{H}}(x)}{d x}
$$

Fontein et al thus estımate that under the conditions of their experıment two thirds of the total imposed current $I=5 \mu$ A flows within $70 \mu \mathrm{m}$ from the edges while the remainder is uniformly distributed in the bulk

This experimental data can be modeled ${ }^{464}$ by means of an integral equation derived by MacDonald et al ${ }^{446}$ for the self-consistent potential profile in an ideal impurity-free sample with $N$ completely filled (spin-split) Landau levels The electron charge density $\rho_{\mathrm{e}}(x)$ in the $2 \mathrm{DEG}$ is given by

$$
\rho_{\mathrm{e}}(x)=-e n_{\mathrm{s}}\left[1-\frac{e l_{\mathrm{m}}^{2}}{h \omega_{\mathrm{c}}} V_{\mathrm{H}}^{\prime \prime}(x)\right]
$$

${ }^{464} \mathrm{C}$ W J Beenakker, unpublished 
This equation follows from the Schrödinger equation in a smoothly varying electrostatic potential, so the factor between brackets is close to unity. Substitution of the net charge density $e n_{\mathrm{s}}+\rho_{\mathrm{e}}(x)$ into the Poisson equation gives $^{446}$

$$
V_{\mathrm{H}}(x)=-\xi \int_{-W / 2}^{+W / 2} d x^{\prime} \ln \left(\frac{2}{W}\left|x-x^{\prime}\right|\right) V_{\mathrm{H}}^{\prime \prime}\left(x^{\prime}\right) .
$$

The characteristic length $\xi \equiv N l_{\mathrm{m}}^{2} / \pi a^{*}$ is defined in terms of the magnetic length $l_{\mathrm{m}}$ and the effective Bohr radius $a^{*} \equiv \varepsilon \hbar^{2} / m e^{2}$ (with $\varepsilon$ the dielectric constant).

The integral equation (18.8) was solved numerically by MacDonald et $a l .{ }^{446}$ and analytically by means of the Wiener-Hopf technique by Thouless. ${ }^{448}$ Here we describe a somewhat simpler approach, ${ }^{464}$ which is sufficiently accurate for the present purpose. For magnetic field strengths in the QHE regime the length $\xi$ is very small. For example, if $N=4, l_{\mathrm{m}}=11.5 \mathrm{~nm}$ (for $B=5 \mathrm{~T}$ ), $a^{*}=10 \mathrm{~nm}$ (for GaAs with $\varepsilon=13 \varepsilon_{0}$ and $m=0.067 m_{\mathrm{c}}$ ), then $\xi=17 \mathrm{~nm}$. It is therefore meaningful to look for a solution of Eq. (18.8) in the limit $\xi \ll W$. The result is that $V_{\mathrm{H}}(x)=$ constant $x \ln |(x-W / 2) /(x+W / 2)|$ if $|x| \leqslant W / 2-\xi$, with a linear extrapolation from $|x|=W / 2-\xi$ to $|x|=W / 2$. One may verify that this is indeed the answer, by substituting the preceding expression into Eq. (18.8) and performing one partial integration. The arbitrary constant in the expression for $V_{\mathrm{H}}$ may be eliminated in favor of the total current $I$ flowing through the Hall bar, by applying Eq. (18.6) to the case of $N$ filled spin-split Landau levels. This gives the final answer

$$
V_{\mathrm{H}}(x)=\frac{1}{2} I R_{\mathrm{H}}\left(1+\ln \frac{W}{\xi}\right)^{-1} \ln \left|\frac{x-W / 2}{x+W / 2}\right|, \quad \text { if }|x| \leqslant \frac{W}{2}-\xi,
$$

with a linear extrapolation of $V_{\mathrm{H}}$ to $\pm \frac{1}{2} I R_{\mathrm{H}}$ in the interval within $\xi$ from the edge. The Hall resistance is $R_{\mathrm{H}}=h / N e^{2}$. The approximation (18.9) is equivalent for small $\xi$ to the analytical solution of Thouless, and is close to the numerical solutions given by MacDonald $e t$ al., even for a relatively large value $\xi / W=0.1$.

In Fig. 80 the result (18.9) has been plotted (solid curve) for the parameters of the experiment by Fontein et al. $\left(\xi / W=0.85 \times 10^{-5}\right.$ for $N=4, B=5 \mathrm{~T}$, and $W=2 \mathrm{~mm}$ ). The agreement with experiment is quite satisfactory in view of the fact that the theory contains no adjustable parameters. The theoretical profile is steeper at the edges than in the experiment, which may be due to the limited experimental resolution of $70 \mu \mathrm{m}$. The total voltage drop between the two edges in the calculation $\left(h I / N e^{2} \approx 32 \mathrm{mV}\right.$ for $I=5 \mu \mathrm{A}$ and $\left.N=4\right)$ agrees with the measured Hall voltage of $\approx 30 \mathrm{mV}$, but the optically determined value of $40 \mathrm{mV}$ is somewhat larger - for a reason that we do not understand. 
We have discussed this topic of the current distribution in the QHE in some detail to convince the reader that the concentration of the potential drop (and hence of the current) near the edges can be understood from the electrostatics of edge charges, but cannot be used to test the validity of a linear response formulation of the QHE in terms of edge states. Indeed, edge states were completely neglected in the foregoing theoretical analysis, which nonetheless captures the essential features of the experiment.

\section{Selective Population and Detection of Edge Channels}

The absence of local equilibrium at the current or voltage contacts leads to anomalies in the quantum Hall effect, unless the contacts are ideal (in the sense that each edge channel at the Fermi level is transmitted through the contact with probability 1 ). Ideal versus disordered contacts are dealt with in Sections 19.a and 19.b. A quantum point contact can be seen as an extreme example of a disordered contact, as discussed in Section 19.c. Anomalies in the Shubnikov-De Haas effect due to the absence of local equilibrium are the subject of Section 19.d.

\section{a. Ideal Contacts}

In a two-terminal measurement of the quantum Hall effect the contact resistances of the current source and drain are measured in series with the Hall resistance. For this reason precision measurements of the QHE are usually performed in a four-terminal measurement configuration, in which the voltage contacts do not carry a current. ${ }^{445}$ Contact resistances then do not play a role, provided that local equilibrium is established near the voltage contacts [or, by virtue of the reciprocity relation (12.16), near the current contacts]. As we mentioned in Section 18, local equilibrium can be grossly violated in the QHE. Accurate quantization then requires that either the current or the voltage contacts are ideal, in the sense that the edge states at the Fermi level have unit transmission probability through the contacts. ${ }^{12}$ In this subsection we return to the four-terminal measurements on a quantum point contact considered in Section 13.b, but now in the QHE regime where the earlier assumption of local equilibrium near the voltage contacts is no longer applicable in general. We assume strong magnetic fields so that the four-terminal longitudinal resistance $R_{\mathrm{L}}$ of the quantum point contact is determined by the potential barrier in the constriction (rather than by its width).

Let us apply the Landauer-Büttiker formalism to the geometry of Fig. 81 . As in Section 13.b, the number of spin-degenerate edge channels in the wide 2DEG and in the constriction are denoted by $N_{\text {wide }}$ and $N_{\min }$, respectively. An ideal contact to the wide $2 \mathrm{DEG}$ perfectly transmits $N_{\text {wide }}$ channels, 


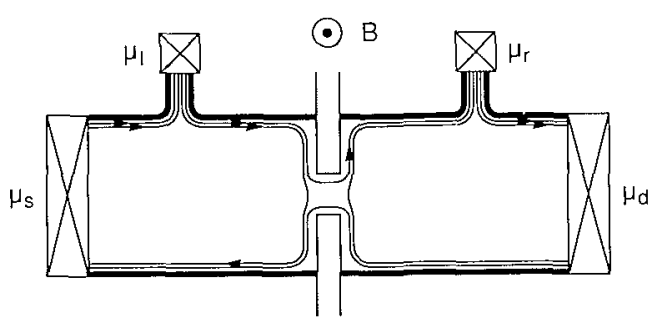

FIG 81 Motion along equipotentials in the QHE regime, in a four-termınal geometry with a saddle-shaped potential formed by a split gate (shaded) Ideal contacts are assumed The thin lines indicate the location of the edge channels at the Fermi level, with the arrows pointing in the direction of motion of edge channels that are populated by the contacts (crossed squares) Taken from $\mathrm{H}$ van Houten et al, in "Nanostructured Systems" (M A Reed, ed) Academıc, New York 1991

whereas the constriction transmits only $N_{\min }$ channels. The remaining $N_{\text {wide }}-N_{\text {min }}$ channels are reflected back along the opposite 2DEG boundary (cf. Fig. 81). We denote by $\mu_{1}$ and $\mu_{\mathrm{r}}$ the chemical potentials of adjacent voltage probes to the left and to the right of the constriction. The current source is at $\mu_{\mathrm{s}}$, and the drain at $\mu_{\mathrm{d}}$. Applying Eq. (12.12) to this case, using $I_{\mathrm{s}}=-I_{\mathrm{d}} \equiv I, I_{\mathrm{r}}=I_{\mathrm{l}}=0$, one finds for the magnetic field direction indicated in Fig. 81,

$$
\begin{aligned}
(h / 2 e) I & =N_{\text {wide }} \mu_{\mathrm{s}}-\left(N_{\text {wide }}-N_{\text {min }}\right) \mu_{\mathrm{l}}, \\
0 & =N_{\text {wide }} \mu_{1}-N_{\text {wide }} \mu_{\mathrm{s}}, \\
0 & =N_{\text {wide }} \mu_{\mathrm{r}}-N_{\text {min }} \mu_{1} .
\end{aligned}
$$

We have used the freedom to choose the zero level of chemical potential by fixing $\mu_{\mathrm{d}}=0$, so we have three independent (rather than four dependent) equations. The two-terminal resistance $R_{2 \mathrm{t}} \equiv \mu_{\mathrm{s}} / e I$ following from Eq. (19.1) is

$$
R_{2 \mathrm{t}}=\frac{h}{2 e^{2}} \frac{1}{N_{\min }}
$$

unaffected by the presence of the additional voltage probes in Fig. 81 . The four-terminal longitudinal resistance $R_{\mathrm{L}} \equiv\left(\mu_{1}-\mu_{\mathrm{r}}\right) / e I$ is

$$
R_{\mathrm{L}}=\frac{h}{2 e^{2}}\left(\frac{1}{N_{\mathrm{min}}}-\frac{1}{N_{\mathrm{wide}}}\right) \text {. }
$$

In the reversed field direction the same result is obtained. Equation (19.3), derived for ideal contacts without assuming local equilibrium near the contacts, is identical to Eq. (13.7), derived for the case of local equilibrium. 


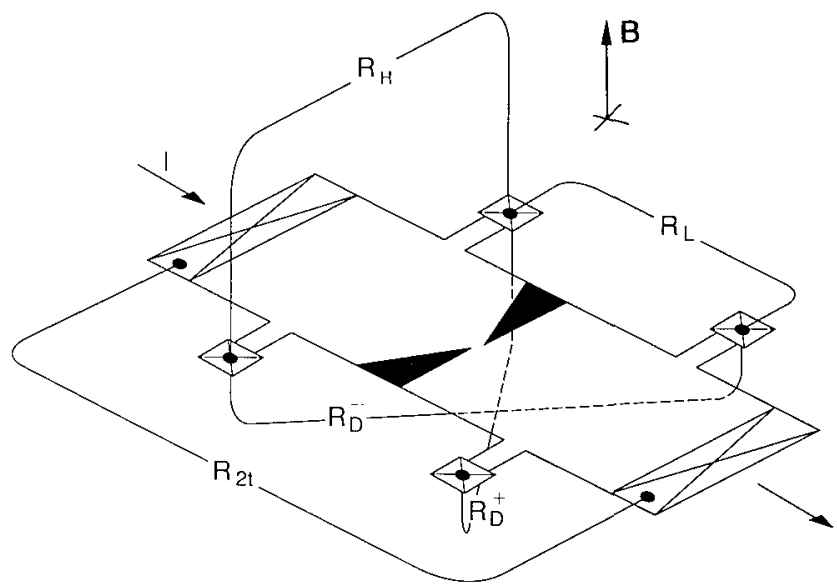

FIG. 82. Perspective view of a six-terminal Hall bar containing a point contact, showing the various two- and four-terminal resistances mentioned in the text. Taken from $\mathrm{H}$. van Houten $e t$ al., in "Nanostructured Systems" (M. A. Reed, ed.). Academic, New York 1991.

In a six-terminal measurement geometry (see Fig. 82), one can also measure the Hall resistance in the wide regions, which is simply $R_{\mathrm{H}}=R_{21}-R_{\mathrm{L}}$ or

$$
R_{\mathrm{H}}=\frac{h}{2 e^{2}} \frac{1}{N_{\text {wide }}},
$$

which is unaffected by the presence of the constriction. This is a consequence of our assumption of ideal voltage probes. One can also measure the two four-terminal diagonal resistances $R_{\mathrm{D}}^{+}$and $R_{\mathrm{D}}^{-}$across the constriction in such a way that the two voltage probes are on opposite edges of the $2 \mathrm{DEG}$, on either side of the constriction (see Fig. 82). Additivity of voltages on contacts tells us that $R_{\mathrm{D}}^{ \pm}=R_{\mathrm{H}} \pm R_{\mathrm{L}}$ (for the magnetic field direction of Fig. 82); thus,

$$
R_{\mathrm{D}}^{+}=\frac{h}{2 e^{2}} \frac{1}{N_{\text {min }}} ; \quad R_{\mathrm{D}}^{-}=\frac{h}{2 e^{2}}\left(\frac{2}{N_{\text {wide }}}-\frac{1}{N_{\text {min }}}\right) \text {. }
$$

On field reversal, $R_{\mathrm{D}}^{+}$and $R_{\mathrm{D}}^{-}$are interchanged. Thus, a four-terminal resistance $\left[R_{\mathrm{D}}^{+}\right.$in Eq. (19.5)] can in principle be equal to the two-terminal resistance $\left[R_{2 t}\right.$ in Eq. (19.2)]. The main difference between these two quantities is that an additive contribution of the ohmic contact resistance (and of a part of the diffusive background resistance in weak magnetic fields) is eliminated in the four-terminal resistance measurement.

The fundamental reason that the assumption of local equilibrium made in Section 13.b (appropriate for weak magnetic fields) and that of ideal contacts 


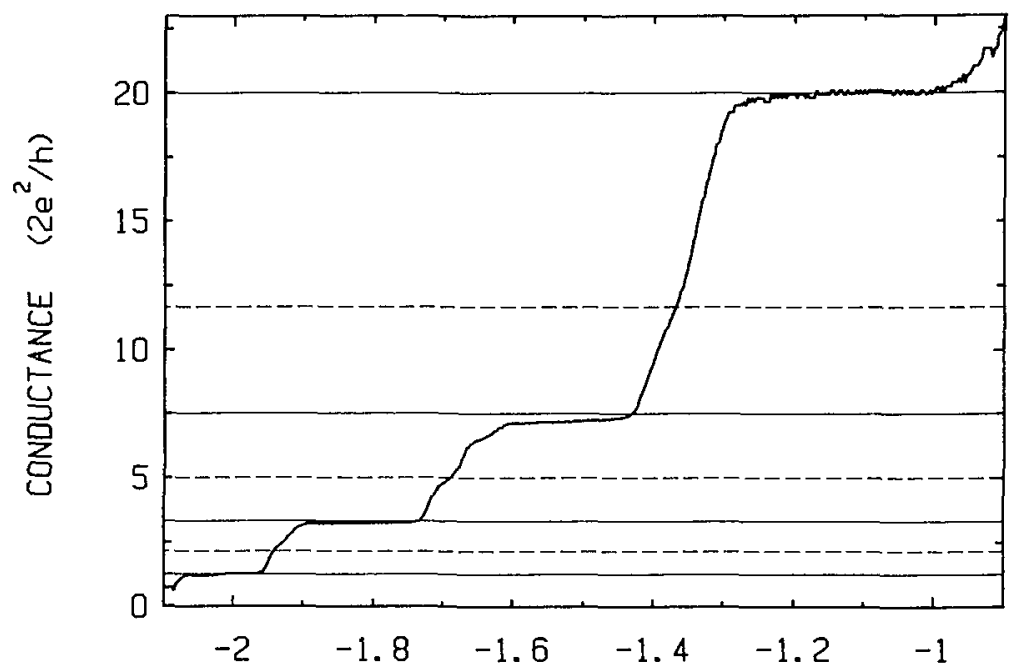

GATE VOLTAGE (V)

FIG 83 "Fractional' quantization in the integer QHE of the four-terminal longitudinal conductance $R_{\mathrm{L}}^{-1}$ of a point contact in a magnetic field of $14 \mathrm{~T}$ at $T=06 \mathrm{~K}$ The solid horizontal lines indicate the quantized plateaus predicted by Eq (193), with $N_{\text {wide }}=5$ and $N_{\text {min }}=1,2,3,4$ The dashed lines give the location of the spin-split plateaux, which are not well resolved at this magnetıc field value Taken from L P Kouwenhoven, Master's thesis, Delft University of Technology, 1988

made in this section (for strong fields) yield identical answers is that an ideal contact attached to the wide 2DEG regions induces a local equilibrium by equipartitioning the outgoing current among the edge channels (This is illustrated in Fig 81, where the current entering the voltage probe to the right of the constriction is carried by a single edge channel, while the equally large current flowing out of that probe is equipartitioned over the two edge channels avallable for transport in the wide region) In weaker magnetic fields, when the cyclotron radius exceeds the width of the narrow 2DEG region connectung the voltage probe to the Hall bar, not all edge channels in the wide 2DEG region are transmitted into the voltage probe (even if it does not contain a potential barrier) This probe is then not effective in equipartitioning the current That is the reason that the weak-field analysis in Section $13 \mathrm{~b}$ required the assumption of a local equilibrium in the wide 2DEG near the contacts

We now discuss some experimental results, which confirm the behavior predicted by Eq (193) in the QHE regime, to complement the weak-field experiments discussed in Section $13 \mathrm{~b}$ Measurements on a quantum point 
contact by Kouwenhoven et al ${ }^{307465}$ in Fig 83 show the quantization of the longitudinal conductance $R_{\mathrm{L}}^{-1}$ in fractions of $2 e^{2} / h$ (for unresolved spin degeneracy) The magnetic field is kept fixed at $14 \mathrm{~T}$ (such that $N_{\text {wide }}=5$ ) and the gate voltage is varied (such that $N_{\min }$ ranges from 1 to 4 ) Conductance plateaux close to $5 / 4,10 / 3,15 / 2$, and $20 \times\left(2 e^{2} / h\right)$ (solid horizontal lines) are observed, in accord with Eq (193) Spin-split plateaux (dashed lines) are barely resolved at this rather low magnetic field Simılar data were reported by Snell et al ${ }^{342}$ Observations of such a "fractional" quantization due to the integer QHE were made before on wide Hall bars with regions of different electron density in series, ${ }^{466} 467$ but the theoretical explanation ${ }^{468}$ given at that time was less straightforward than Eq (193)

In the high-field regime the point contact geometry of Fig 81 is essentially equivalent to a geometry in which a potential barrier is present across the entıre width of the Hall bar (created by means of a narrow contınuous gate) The latter geometry was studied by Haug et al ${ }^{340}$ and by Washburn et al ${ }^{339}$ The geometries of both experıments ${ }^{339} 340$ are the same (see Figs 84 and 85), but the results exhibit some interesting differences because of the different dimensions of gate and channel Hauge et al ${ }^{340}$ used a sample of macroscopic dimensions, the channel width being $100 \mu \mathrm{m}$ and the gate length 10 and $20 \mu \mathrm{m}$ Results are shown in Fig 84 As the gate voltage is varied, a quantized plateau at $h / 2 e^{2}$ is seen in the longitudinal resistance at fixed magnetic field, in agreement with Eq (193) (the plateau occurs for two spinsplit Landau levels in the wide region and one spin-split level under the gate) A qualitatively different aspect of the data in Fig 84, compared with Fig 83, 1s the presence of a resistance minımum Equation (19 3), in contrast, predicts that $R_{\mathrm{L}}$ varies monotonically with barrier height, and thus with gate voltage A model for the effect has been proposed in a different paper by Haug et al ${ }^{341}$ based on a competition between backscattering and tunneling through localized states in the barrier region They find that edge states that are totally reflected at a given barrier height may be partially transmitted if the barrier height is further increased The importance of tunneling is consistent with the increase of the amplitude of the dip as the gate length is reduced from 20 to $10 \mu \mathrm{m}$ A related theoretical study was performed by Zhu et al ${ }^{469}$

\footnotetext{
${ }^{465} \mathrm{~L} P$ Kouwenhoven, Master's thesis, Delft University of Technology, 1988

${ }^{466} \mathrm{~K}$ von Klitzing, $\mathrm{G}$ Ebert, $\mathrm{N}$ Kleinmichel, $\mathrm{H}$ Obloh, $\mathrm{G}$ Dorda, and $\mathrm{G}$ Wermann, "Proc ICPS 17" (J D Chad 1 and W A Harrison, eds) Springer, New York, 1985

${ }^{467}$ D A Syphers, F F Fang, and P J Strles, Surf Scl 142, 208 (1984), F F Fang and P J Stıles, Phys Rev B 27, 6487 (1983), F F Fang and P J Stiles, Phys Rev B 29, 3749 (1984) A B Berkut, Yu V Dubrovskı, M S Nunuparov, M I Reznıkov, and V I Tal'yanskı, Pts'ma $Z h$ Teor Ftz 44, 252 (1986) [JETP Lett 44, 324 (1986)]

${ }^{468}$ D A Syphers and P J Strles, Phys Rev B 32, 6620 (1985)

${ }^{469}$ Y Zhu, J Shı, and S Feng, Phys Rev B 41, 8509 (1990)
} 

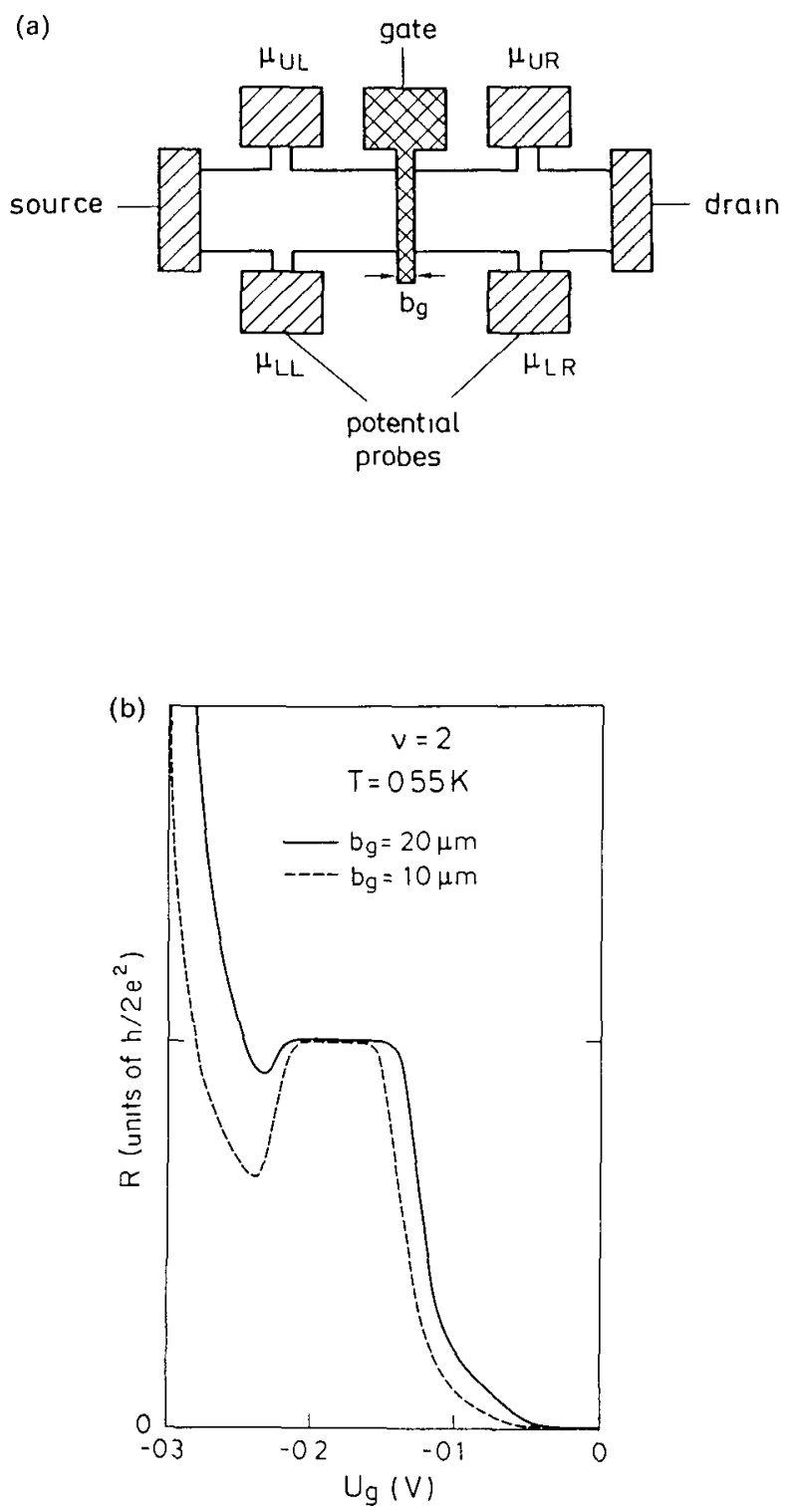

FIG 84 (a) Schematic view of a wide Hall bar contaning a potential barrier imposed by a gate electrode of length $b_{8}$ (b) Longitudınal resistance as a function of gate voltage in the QHE regime (two spin-split Landau levels are occupied in the unperturbed electron gas regions) The plateau shown is at $R_{\mathrm{L}}=h / 2 e^{2}$, in agreement with Eq (193) Results for $b_{\mathrm{g}}=10 \mu \mathrm{m}$ and $20 \mu \mathrm{m}$ are compared A pronounced dip develops in the device with the shortest gate length Taken from $\mathrm{R}$ J Haug et al, Phys Rev B 39, 10892 (1989) 
(a)

4

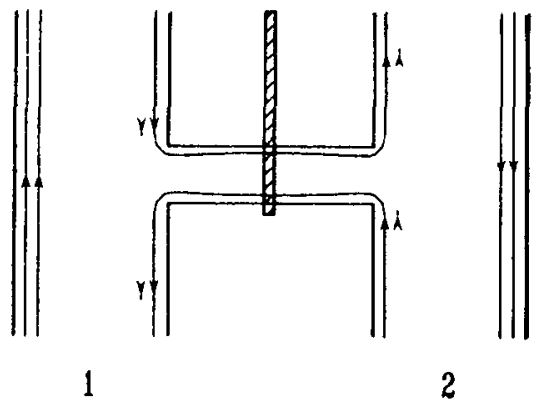

(b)

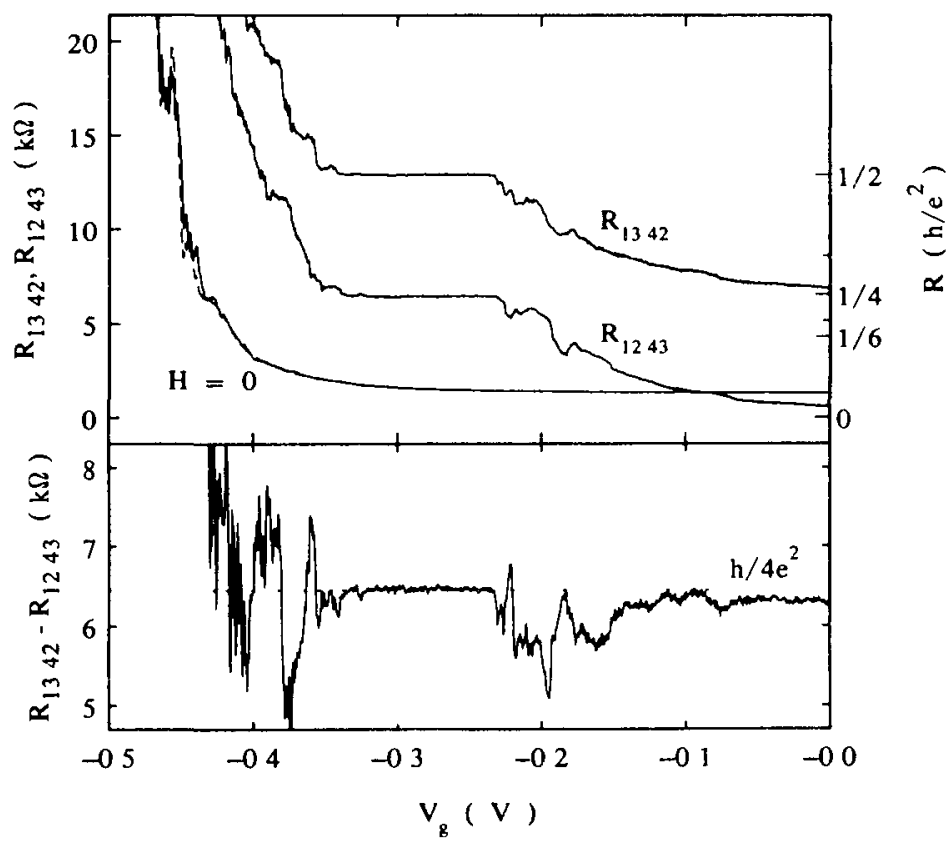

FIG 85 (a) Schematic view of a $2-\mu$ m-wide channel containing a potential barrier imposed by a $01-\mu \mathrm{m}$-long gate (b) Top diagonal resistance $R_{1342} \equiv R_{\mathrm{D}}^{+}$and longitudinal resistance $R_{1243} \equiv R_{\mathrm{L}}$ as a function of gate voltage in a strong magnetic field $(B=52 \mathrm{~T})$, showing a quantized plateau in agreement with Eqs (195) and (193), respectively For comparison also the two zero-field traces are shown, which are almost identical Bottom Difference $R_{\mathrm{D}}^{+}-R_{\mathrm{L}}=R_{\mathrm{H}}$ at $52 \mathrm{~T}$ A normal quantum Hall plateau is found, with oscillatory structure superimposed in gate voltage regions where $R_{\mathrm{D}}^{+}$and $R_{\mathrm{L}}$ are not quantized Taken from $\mathrm{S}$ Washburn et al, Phys Rev Lett 61, 2801 (1988) 
Washburn et al $^{339}$ studied the longitudinal resistance of a barrier defined by a $01-\mu \mathrm{m}$-long gate across a $2-\mu \mathrm{m}$-wide channel The relevant dimensions are thus nearly two orders of magnitude smaller than in the experiment of Haug et al Again, the resistance is studied as a function of gate voltage at fixed magnetic field The longitudinal $\left(R_{\mathrm{L}} \equiv R_{1243}\right)$ and diagonal $\left(R_{\mathrm{D}}^{+} \equiv R_{1342}\right)$ resistances are shown in Fig 85 , as well as their difference [which according to Eqs (193) and (19 5) would equal the Hall resistance $R_{\mathrm{H}}$ ] In this small sample the quantized plateaux predicted by Eq (193) are clearly seen, but the resistance dips of the large sample of Haug et al are not We recall that resistance dips were not observed in the quantum point contact experiment of Fig 83 either The model of Haug et al ${ }^{341}$ would imply that localized states do not form in barriers of small area Washburn et al find weak resistance fluctuations in the gate voltage intervals between quantized plateaux These fluctuations are presumably due to some form of quantum interference, but have not been further identified

Related experiments on the quantum Hall effect in a 2DEG with a potential barrier have been performed by Hirat et al and by Komiyama et $a l^{427470-472}$ These studies have focused on the role of nonideal contacts in the QHE, which is the subject of the next subsection

\section{b Disordered Contacts}

The validity of Eqs (192)-(195) in the QHE regime breaks down for nonideal contacts if local equilibrium near the contacts is not established The treatment of Section 19 a for ideal contacts implies that the Hall voltage over the wide 2DEG regions adjacent to the constriction is unaffected by the presence of the constriction or potential barrier Experiments by Komiyama et al ${ }^{427472}$ have demonstrated that this is no longer true if one or more contacts are disordered The analysis of their experiments is rather involved, ${ }^{472}$ which is why we do not give a detaled discussion here Instead we review a different experiment, ${ }^{113}$ which shows a deviating Hall resistance in a sample with a constriction and a single disordered contact This experiment can be analyzed in a relatively simple way, ${ }^{307}$ following the work of Buttıker ${ }^{112}$ and Komiyama et al ${ }^{427472}$

The sample geometry is that of Fig 82 In Fig 86 the four-terminal longitudinal resistance $R_{\mathrm{L}}$ and Hall resistance $R_{\mathrm{H}}$ are shown for both a small voltage $(-03 \mathrm{~V})$ and a large voltage $(-25 \mathrm{~V})$ on the gate defining the constriction The longitudinal resistance decreases in weak fields because of

${ }^{470} \mathrm{H}$ Hiral, S Komiyama, S Hiyamizu, and S Sasa in "Proc ICPS 19," p 55 (W Zawadaskı, ed) Institute of Physics, Polish Academy of Sciences, 1988

${ }^{471}$ S Komiyama, H Hiral, S Sasa, and T Fuj1, Soltd State Comm 73, 91 (1990), H Hira1, S Komiyama, S Sasa, and T Fuj11, J Phys Soc Jap 58, 4086 (1989)

${ }^{472}$ S Komiyama and H Hira1, Phys Rev B 40, 7767 (1989) 


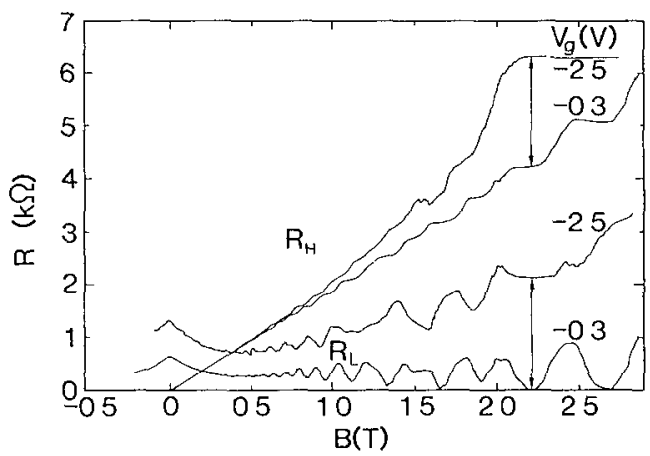

Fig 86 Nonvanıshing Shubnıkov-De Haas minıma in the longitudinal resistance $R_{\mathrm{L}}$ and anomalous quantum Hall resistance $R_{\mathrm{H}}$, measured in the point contact geometry of $F_{1} g 82$ at $50 \mathrm{mK}$ These experimental results are extensions to higher fields of the weak-field traces shown in Fig 50 The Hall resistance has been measured across the wide region, more than $100 \mu \mathrm{m}$ away from the constriction, yet $R_{\mathrm{H}}$ is seen to increase of the gate voltage is raised from $-03 \mathrm{~V}$ to $-25 \mathrm{~V}$ The magnitude at $B=22 \mathrm{~T}$ of the deviation in $R_{\mathrm{H}}$ and of the Shubnikov-De Haas minimum in $R_{\mathrm{L}}$ are indicated by arrows, which both for $R_{\mathrm{H}}$ and $R_{\mathrm{L}}$ have a length of $\left(h / 2 e^{2}\right)$ $\left(\frac{1}{2}-\frac{1}{3}\right)$, in agreement with the analysis given in the text Taken from $\mathbf{H}$ van Houten et al, in "Nanostructured Systems" (M A Reed, ed) Academıc, New York, 1991

reduction of backscattering, as discussed in Section 13.b. At larger fields Shubnikov-De Haas oscillations develop. The data for $V_{\mathrm{g}}=-0.3 \mathrm{~V}$ exhibit zero minima in the Shubnikov-De Haas oscillations in $R_{\mathrm{L}}$ and the normal quantum Hall resistance $R_{\mathrm{H}}=\left(h / 2 e^{2}\right) N_{\text {wide }}^{-1}$, determined by the number of Landau levels occupied in the wide regions $\left(N_{\text {wide }}\right.$ can be obtained from the quantum Hall effect measured in the absence of the constriction or from the periodicity of the Shubnikov-De Haas oscillations).

At the higher gate voltage $V_{\mathrm{g}}=-2.5 \mathrm{~V}$, nonvanıshıng minima in $R_{\mathrm{L}}$ are seen in Fig. 86 as a result of the formation of a potential barrier in the constriction. At the minıma, $R_{\mathrm{L}}$ has the fractional quantization predicted by Eq. (19.3). For example, the plateau in $R_{\mathrm{L}}$ around $2.2 \mathrm{~T}$ for $V_{\mathrm{g}}=-2.5 \mathrm{~V}$ is observed to be at $R_{\mathrm{L}}=2.1 \mathrm{k} \Omega \approx\left(h / 2 e^{2}\right) \times\left(\frac{1}{2}-\frac{1}{3}\right)$, in agreement with the fact that the two-terminal resistance yields $N_{\mathrm{m} n \mathrm{n}}=2$ and the number of Landau levels in the wide regions $N_{\text {wide }}=3$. In spite of this agreement, and in apparent conflict with Eq. (19.4), the Hall resistance $R_{\mathrm{H}}$ has increased over its value for small gate voltages. Indeed, around $2.2 \mathrm{~T}$ a Hall plateau at $R_{\mathrm{H}}=6.3 \mathrm{k} \Omega \approx\left(h / 2 e^{2}\right) \times \frac{1}{2}$ is found for $V_{\mathrm{g}}=-2.5 \mathrm{~V}$, as if the number of occupred Landau levels was given by $N_{\text {min }}=2$ rather than by $N_{\text {wide }}=3$. This unexpected deviation was noted in Ref. 113, but was not understood at the time. At higher magnetic fields (not shown in Fig. 86) the $N=1$ plateau is reached, and the deviation in the Hall resistance vanishes.

As pointed out in Ref. 307, the likely explanation of the data of Fig. 86 is 


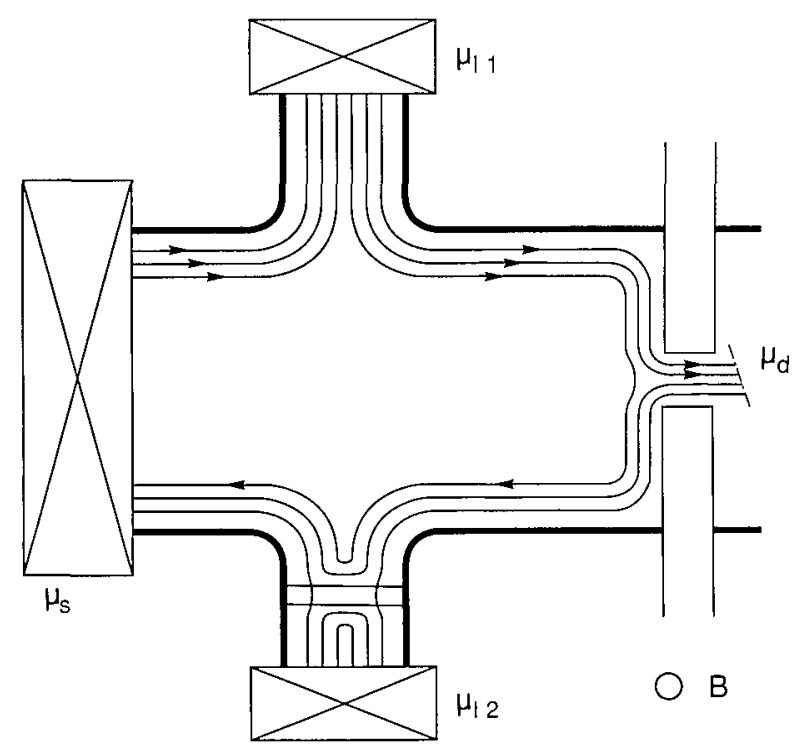

Fig 87 Illustration of the flow of edge channels along equipotentials in a sample with a constriction (defined by the shaded gates) and a disordered voltage probe (a potential barrier in the probe is indicated by the shaded bar) Taken from $\mathrm{H}$ van Houten $e t$ al, in "Nanostructured Systems" (M A Reed, ed) Academıc, New York

that one of the ohmic contacts used to measure the Hall voltage is disordered in the sense of Büttiker ${ }^{112}$ that not all edge channels have unit transmission probability into the voltage probe. The disordered contact can be modeled by a potential barrier in the lead with a height not below that of the barrier in the constriction, as illustrated in Fig. 87. A net current $I$ flows through the constriction, determined by its two-terminal resistance according to $I=(2 e / h) N_{\min } \mu_{\mathrm{s}}$, with $\mu_{\mathrm{s}}$ the chemical potential of the source reservoir (the chemical potential of the drain reservoir $\mu_{\mathrm{d}}$ is taken as a zero reference). Equation (12.12) applied to the two opposite Hall probes $l_{1}$ and $l_{2}$ in Fig. 87 takes the form (using $I_{l_{1}}=I_{l_{2}}=0, \mu_{\mathrm{s}}=(h / 2 e) I / N_{\operatorname{man}}$, and $\mu_{\mathrm{d}}=0$ )

$$
\begin{aligned}
& 0=N_{\text {wide }} \mu_{l_{1}}-T_{s \rightarrow l_{1}} \frac{h}{2 e} \frac{I}{N_{\min }}-T_{l_{2} \rightarrow l_{1}} \mu_{l_{2}}, \\
& 0=N_{l_{2}} \mu_{l_{2}}-T_{\mathrm{s} \rightarrow l_{2}} \frac{h}{2 e} \frac{I}{N_{\min }}-T_{l_{1} \rightarrow l_{2}} \mu_{l_{1}},
\end{aligned}
$$

where we have assumed that the disordered Hall probe $l_{2}$ transmits only $N_{l_{2}}<N_{\text {wide }}$ edge channels because of the barrier in the lead. For the field direction shown in Fig. 87 one has, under the assumption of no interedge 
channel scattering from constriction to probe $l_{2}, T_{\mathrm{s} \rightarrow l_{1}}=N_{\text {wide }}$, $T_{\mathrm{s} \rightarrow l_{2}}=T_{l_{2} \rightarrow l_{1}}=0$, and $T_{l_{1} \rightarrow l_{2}}=\max \left(0, N_{l_{2}}-N_{\min }\right)$ Equation $(196)$ then leads to a Hall resistance $R_{\mathrm{H}} \equiv\left(\mu_{l_{1}}-\mu_{l_{2}}\right) / e I$ given by

$$
R_{\mathrm{H}}=\frac{h}{2 e^{2}} \frac{1}{\max \left(N_{l_{2}}, N_{\mathrm{min}}\right)}
$$

In the opposite field direction the normal Hall resistance $R_{\mathrm{H}}=\left(h / 2 e^{2}\right) N_{\text {wide }}^{-1}$ is recovered

The assumption of a single disordered probe, plus absence of interedge channel scattering from constriction to probe, thus explains the observation in Fig 86 of an anomalously high quantum Hall resistance for large gate voltages, such that $N_{\mathrm{min}}<N_{\text {wide }}$ Indeed, the experimental Hall resistance for $V_{\mathrm{g}}=-25 \mathrm{~V}$ has a plateau around $22 \mathrm{~T}$ close to the value $R_{\mathrm{H}}=\left(h / 2 e^{2}\right) N_{\min }^{-1}$ (with $N_{\min }=2$ ), in agreement with Eq (197) if $N_{l_{2}} \leqslant N_{\min }$ at this gate voltage This observation demonstrates the absence of interedge channel scattering over $100 \mu \mathrm{m}$ (the separation of constriction and probe), but only between the highest-1ndex channel (with index $n=N_{\text {wide }}=3$ ) and the two lower-1ndex channels Since the $n=1$ and $n=2$ edge channels are either both empty or both filled ( $\mathrm{cf}$ Fig 87, where these two edge channels lie closest to the sample boundary), any scattering between $n=1$ and 2 would have no measurable effect on the resistances As discussed in Section $19 \mathrm{c}$, we know from the work of Alphenaar et al ${ }^{429}$ that (at least in the present samples) the edge channels with $n \leqslant N_{\text {wide }}-1$ do in fact equilibrate to a large extent on a length scale of $100 \mu \mathrm{m}$

In the absence of a constriction, or at small gate voltages (where the constriction is just defined), one has $N_{\text {min }}=N_{\text {wide }}$ so that the normal Hall effect is observed in both field directions This is the situation realized in the experimental trace for $V_{\mathrm{g}}=-03 \mathrm{~V}$ in Fig 86 In very strong fields such that $N_{\text {min }}=N_{t_{2}}=N_{\text {wide }}=1$ (still assuming nonresolved spin splitting), the normal result $R_{\mathrm{H}}=h / 2 e^{2}$ would follow even if the contacts contain a potential barrier, in agreement with the experiment (not shown in Fig 86) This is a more general result, which holds also for a barrier that only partially transmits the $n=1$ edge channel ${ }^{112}$

A simılar analysis as the foregoing predicts that the longitudinal resistance measured on the edge of the sample that contains ideal contacts retains its regular value (193) On the opposite sample edge the measurement would involve the disordered contact, and one finds instead

$$
R_{\mathrm{L}}=\frac{h}{2 e^{2}}\left(\frac{1}{N_{\min }}-\frac{1}{\max \left(N_{l_{2}}, N_{\mathrm{min}}\right)}\right)
$$

${ }^{473}$ U Sivan, Y Imry, and C Hartzstein, Phys Rev B 39, 1242 (1989)

${ }^{474} \mathrm{U}$ Sivan and Y Imry, Phys Rev Lett 61, 1001 (1988)

${ }^{475}$ M Buttıker, Phys Rev B 38, 12724 (1988) 
for the field direction shown in Fig 87, while Eq (193) is recovered for the other field direction The observation in the experiment of Fig 86 for $V_{\mathrm{g}}=-25 \mathrm{~V}$ of a regular longitudinal resistance [in agreement with $\mathrm{Eq}$ (193)], along with an anomalous quantum Hall resistance is thus consistent with this analysis

The experiments ${ }^{426} 429$ discussed in the following subsection are topologically equivalent to the geometry of $\mathrm{Fig} 87$, but involve quantum point contacts rather than ohmic contacts This gives the possibility of populating and detecting edge channels selectively, thereby enabling a study of the effects of a nonequilibrium population of edge channels in a controlled manner

\section{c Quantum Point Contacts}

In Section 14 we have seen how a quantum point contact can inject a coherent superposition of edge channels at the 2DEG boundary, in the coherent electron focusing experiment ${ }^{59}$ In that section we restricted ourselves to weak magnetic fields Here we discuss the experiment by van Wees et al, ${ }^{426}$ which shows how in the QHE regime the point contacts can be operated in a different way as selective injectors (and detectors) of edge channels We recall that electron focusing can be measured as a generalized Hall resistance, in which case the pronounced peaked structure due to mode interference is superimposed on the weak-field Hall resistance (cf Fig 53) If the weak-field electron-focusing experiments are extended to stronger magnetic fields, a transition is observed to the quantum Hall effect, provided the injecting and detecting point contacts are not too strongly pinched off ${ }^{59}$ The oscillations characteristic of mode interference disappear in this field regime, suggesting that the coupling of the edge channels (which form the propagating modes from injector to collector) is suppressed, and adiabatic transport is realized It is now no longer sufficient to model the point contacts by a point source-detector of infinitesimal width (as was done in Section 14), but a somewhat more detalled description of the electrostatic potential $V(x, y)$ defining the point contacts and the 2DEG boundary between them is required Schematically, $V(x, y)$ is represented in Fig 88a Fringing fields from the split gate create a potential barrier in the point contacts, so $V$ has a saddle form as shown The heights of the barriers $E_{1}, E_{\mathrm{c}}$ in the injector and collector are separately adjustable by means of the voltages on the split gates and can be determined from the two-terminal conductances of the individual point contacts The point contact separation in the experiment of Ref 426 is small $(15 \mu \mathrm{m})$, so one can assume fully adrabatic transport from injector to collector in strong magnetic fields As discussed in Section 18, adiabatic transport is along equipotentials at the guiding center energy $E_{\mathrm{G}}$ Note that the edge channel with the smallest index $n$ has the largest guiding center energy [according to $\mathrm{Eq}(181)$ ] In the absence of interedge channel 

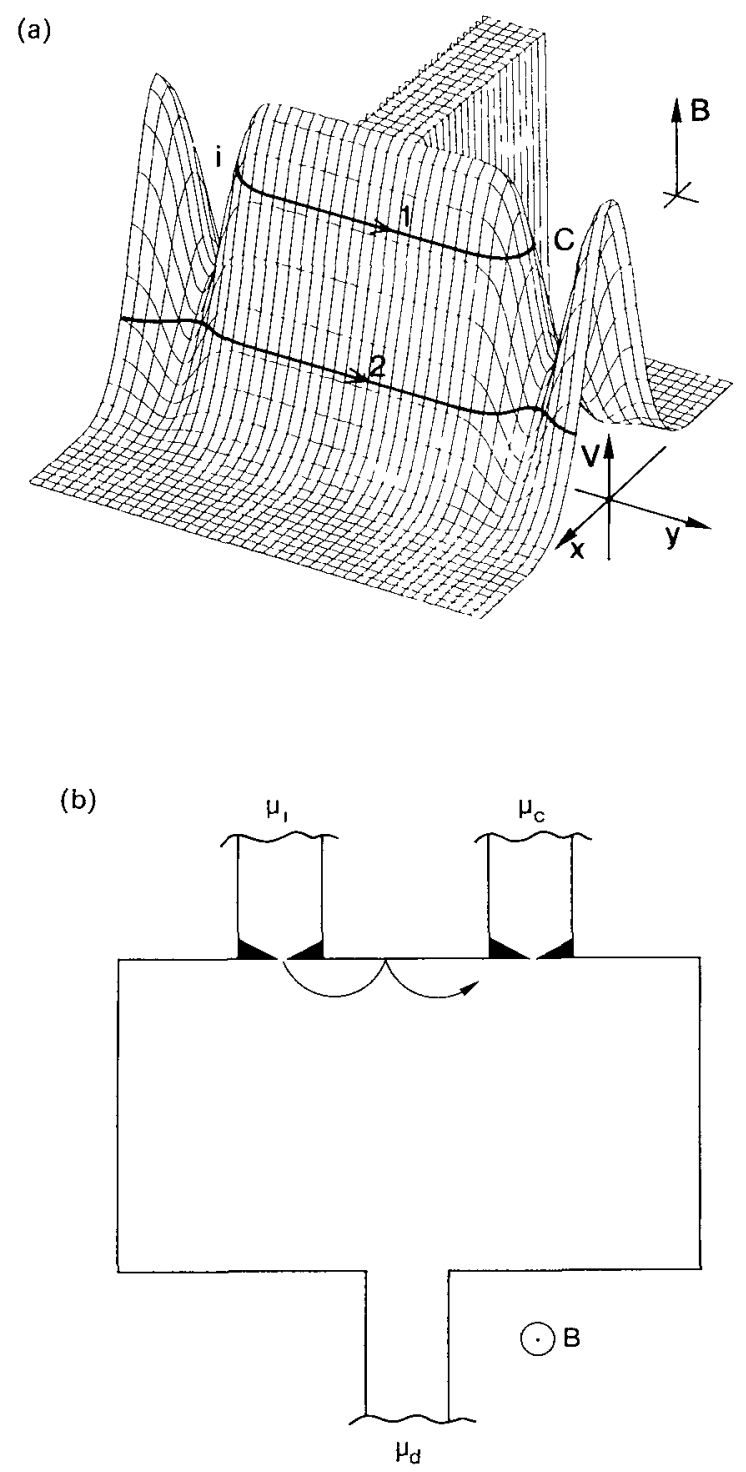

FIG. 88. (a) Schematic potential landscape, showing the 2DEG boundary and the saddleshaped injector and collector point contacts. In a strong magnetic field the edge channels are extended along equipotentials at the guiding center energy, as indicated here for edge channels with index $n=1,2$ (the arrows point in the direction of motion). In this case a Hall conductance of $\left(2 e^{2} / h\right) N$ with $N=1$ would be measured by the point contacts, in spite of the presence of two occupied spin-degenerate Landau levels in the bulk 2DEG. Taken from C. W. J. Beenakker et al., Festkórperprobleme 29, 299 (1989). (b) Three-terminal conductor in the electron focusing geometry. Taken from H. van Houten et al., Phys. Rev. B. 39, 8556 (1989). 
scattering, edge channels can only be transmitted through a point contact if $E_{\mathrm{G}}$ exceeds the potential barrier height (disregarding tunneling through the barrier) The injector thus injects $N_{1} \approx\left(E_{\mathrm{F}}-E_{1}\right) / \hbar \omega_{\mathrm{c}}$ edge channels into the 2DEG, while the collector is capable of detecting $N_{\mathrm{c}} \approx\left(E_{\mathrm{F}}-E_{\mathrm{c}}\right) / \hbar \omega_{\mathrm{c}}$ channels Along the boundary of the 2DEG, however, a larger number of $N_{\text {widc }} \approx E_{\mathrm{F}} / \hbar \omega_{\mathrm{c}}$ edge channels, equal to the number of occupied bulk Landau levels in the 2DEG, are avallable for transport at the Fermi level The selective population and detection of Landau levels leads to deviations from the normal Hall resistance

These considerations can be put on a theoretical basis by applying the Landauer-Buttiker formalism discussed in Section 12 to the electronfocusing geometry ${ }^{80}$ We consider a three-terminal conductor as shown in Fig $88 \mathrm{~b}$, with point contacts in two of the probes (1njector 1 and collector $\mathrm{c}$ ), and a wide ideal drain contact $d$ The collector acts as a voltage probe, drawing no net current, so that $I_{\mathrm{c}}=0$ and $I_{\mathrm{d}}=-I_{1}$. The zero of energy is chosen such that $\mu_{\mathrm{d}}=0$ One then finds from Eq (1212) the two equations

$$
\begin{aligned}
0 & =\left(N_{\mathrm{c}}-R_{\mathrm{c}}\right) \mu_{\mathrm{c}}-T_{\mathrm{t} \rightarrow \mathrm{c}} \mu_{1}, \\
(h / 2 e) I_{1} & =\left(N_{1}-R_{1}\right) \mu_{1}-T_{\mathrm{c} \rightarrow 1} \mu_{\mathrm{c}},
\end{aligned}
$$

and obtains for the ratio of collector voltage $V_{\mathrm{c}}=\mu_{\mathrm{c}} / e$ (measured relative to the voltage of the current drain) to injected current $I_{1}$ the result

$$
\frac{V_{\mathrm{c}}}{I_{\mathrm{l}}}=\frac{2 e^{2}}{h} \frac{T_{\mathrm{i} \rightarrow \mathrm{c}}}{G_{1} G_{\mathrm{c}}-\delta}
$$

Here $\delta \equiv\left(2 e^{2} / h\right)^{2} T_{1 \rightarrow \mathrm{c}} T_{\mathrm{c} \rightarrow 1}$, and $G_{1} \equiv\left(2 e^{2} / h\right)\left(N_{1}-R_{1}\right), G_{\mathrm{c}} \equiv\left(2 e^{2} / h\right)\left(N_{\mathrm{c}}-R_{\mathrm{c}}\right)$ denote the conductances of injector and collector point contact

For the magnetic field direction indicated in Fig 88, the term $\delta$ in Eq (19 10) can be neglected since $T_{\mathrm{c} \rightarrow 1} \approx 0$ [the resulting Eq (142) was used in Section 14] An additional simplification is possible in the adiabatic transport regime We consider the case that the barrier in one of the two point contacts is sufficiently higher than in the other, to ensure that electrons that are transmitted over the highest barrier will have a negligible probability of being reflected at the lowest barrier Then $T_{1 \rightarrow c}$ is dominated by the transmission probability over the highest barrier, $T_{1 \rightarrow \mathrm{c}} \approx \min \left(N_{1}-R_{\mathrm{l}}, N_{\mathrm{c}}-R_{\mathrm{c}}\right)$ Substitution in Eq (19 10) gives the remarkable result ${ }^{426}$ that the Hall conductance $G_{\mathrm{H}} \equiv I_{1} / V_{\mathrm{c}}$ measured in the electron focusing geometry can be expressed entrely in terms of the contact conductances $G_{1}$ and $G_{\mathrm{c}}$

$$
G_{\mathbf{H}} \approx \max \left(G_{1}, G_{\mathrm{c}}\right)
$$

Equation (1911) tells us that quantized values of $G_{\mathbf{H}}$ occur not at $\left(2 e^{2} / h\right) N_{\text {wide }}$, as one would expect from the $N_{\text {wide }}$ populated Landau levels in 


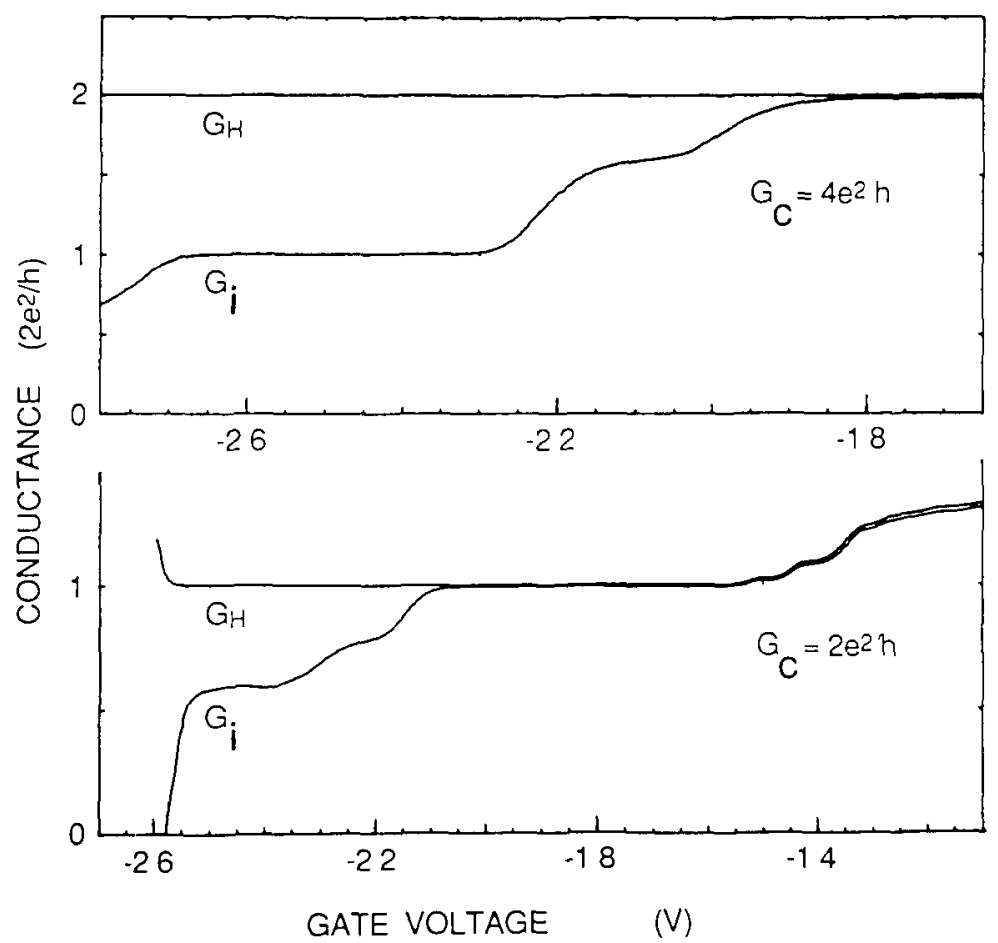

FiG. 89. Experimental correlation between the conductances $G_{1}, G_{c}$ of injector and collector, and the Hall conductance $G_{\mathrm{H}} \equiv I_{1} / V_{\mathrm{c}}$, shown to demonstrate the validity of Eq. (19.11) ( $T=13 \mathrm{~K}$, point contact separation $1 \mathrm{~s} 1.5 \mu \mathrm{m}$ ) The magnetic field was kept fixed (top: $B=2.5 \mathrm{~T}$, bottom $B=38 \mathrm{~T}$, corresponding to a number of occupied bulk Landau levels $N=3$ and 2 , respectively) By increasing the gate voltage on one half of the split-gate defining the injector, $G_{1}$ was varied at constant $G_{\mathrm{c}}$. Taken from B J van Wees et al, Phys. Rev Lett. 62, 1181 (1989).

the 2DEG but at the smaller value of $\left(2 e^{2} / h\right) \max \left(N_{1}, N_{\mathrm{c}}\right)$. As shown in Fig. 89 this is indeed observed experimentally. ${ }^{426}$ Notice in particular how any deviation from quantization in $\max \left(G_{1}, G_{\mathrm{c}}\right)$ is faithfully reproduced in $G_{\mathrm{H}}$, in complete agreement with Eq. (19.11).

The experiment of Ref. 426 was repeated by Alphenaar et al. ${ }^{429}$ for much larger point contact separations $(\approx 100 \mu \mathrm{m})$, allowing a study of the length scale for equilibration of edge channels at the 2DEG boundary. Even after such a long distance, no complete equilibration of the edge channels was found, as manifested by a dependence of the Hall resistance on the gate voltage used to vary the number of edge channels transmitted through the point contact voltage probe (see Fig. 90). As discussed in Section 18.b, a dependence of the resistance on the properties of the contacts is only possible in the absence of local equilibrium. In contrast to the experiment by van Wees 


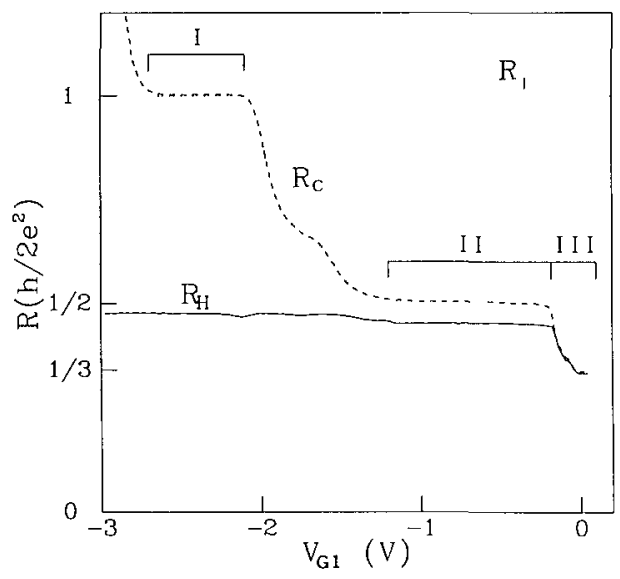

FIG. 90. Results of an experiment similar to that of Fig. 89, but with a much larger separation of $80 \mu \mathrm{m}$ between injector and collector. Shown are $R_{1}=G_{1}^{-1}, R_{\mathrm{c}}=G_{\mathrm{c}}^{-1}$, and $R_{\mathrm{H}}=G_{\mathrm{H}}^{-1}$ as a function of the gate voltage on the collector. $(T=0.45 \mathrm{~K}, B=2.8 \mathrm{~T}$; the normal quantized Hall resistance is $\frac{1}{3}\left(h / 2 e^{2}\right)$.) Regimes I, II, and III are discussed in the text. Taken from B. W. Alphenaar et al., Phys. Rev. Lett. 64, 677 (1990).

et al., ${ }^{426}$ and in disagreement with Eq. (19.11), the Hall resistance in Fig. 90 does not simply follow the smallest of the contact resistances of current and voltage probe. This implies that the assumption of fully adiabatic transport has broken down on a length scale of $100 \mu \mathrm{m}$.

In the experiment a magnetic field was applied such that three edge channels were available at the Fermi level. The contact resistance of the injector was adjusted to $R_{1}=h / 2 e^{2}$, so current was injected in a single edge channel $(n=1)$ only. The gate voltage defining the collector point contact was varied. In Fig. 90 the contact resistances of injector $\left(R_{\mathrm{i}}\right)$ and collector $\left(R_{\mathrm{c}}\right)$ are plotted as a function of this gate voltage, together with the Hall resistance $R_{\mathrm{H}}$. At zero gate voltage the Hall resistance takes on its normal quantized value $\left[R_{\mathrm{H}}=\frac{1}{3}\left(h / 2 e^{2}\right)\right]$. On increasing the negative gate voltage three regions of interest are traversed (labeled III to I in Fig. 90). In region III edge channels 1 and 2 are completely transmitted through the collector, but the $n=3$ channel is partially reflected. In agreement with Eq. (19.11), $R_{\mathrm{H}}$ increases following $R_{\mathrm{c}}$. As region II is entered, $R_{\mathrm{H}}$ levels off while $R_{\mathrm{c}}$ continues to increase up to the $\frac{1}{2}\left(h / 2 e^{2}\right)$ quantized value. The fact that $R_{\mathrm{H}}$ stops slightly short of this value proves that some scattering between the $n=3$ and $n=1,2$ channels has occurred. On increasing the gate voltage further, $R_{\mathrm{c}}$ rises to $h / 2 e^{2}$ in region I. However, $R_{\mathrm{H}}$ shows hardly any increase with respect to its value in region II. This demonstrates that the $n=2$ and $n=1$ edge channels have almost fully equilibrated. A quantitative analysis ${ }^{429}$ shows that, in fact, $92 \%$ of the current originally injected into the $n=1$ edge channel is 


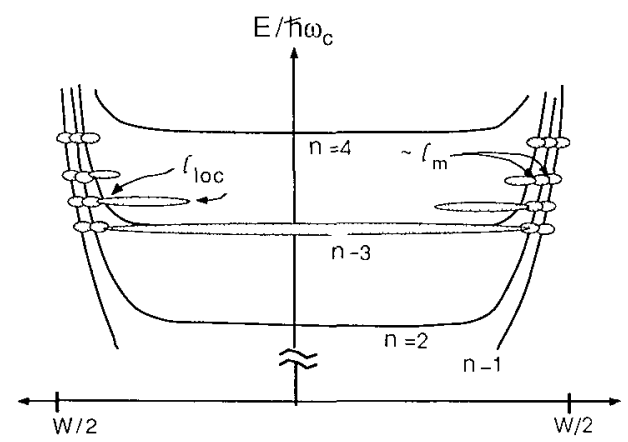

FIG 91 Illustration of the spatial extension (shaded ellipsoids) of edge channels for four different values of the Fermı energy The $n=3$ edge channel can penetrate into the bulk by hybridizıng with the $n=3$ bulk Landau level, coexisting at the Fermı level. This would explain the absence of equilibration between the $n=3$ and $n=1,2$ edge channels. The penetration depth $l_{\text {loc }}$ and the magnetıc length are indicated. Taken from B W. Alphenaar et ai., Phys Rev Lett. 64, $677(1990)$

redistributed equally over the $n=1$ and $n=2$ channels, whereas only $8 \%$ is transferred to the $n=3$ edge channel. The suppression of scattering between the highest-index $n=N$ edge channel and the group of edge channels with $n \leqslant N-1$ was found to exist only if the Fermi level lies in (or near) the $N$ th bulk Landau level. As a qualitative explanation it was suggested ${ }^{429,476}$ that the $N$ th edge channel hybridizes with the $N$ th bulk Landau level when both types of states coexist at the Fermi level. Such a coexistence does not occur for $n \leqslant N-1$ if the potential fluctuations are small compared with $\hbar \omega^{\mathrm{c}}$ (cf. Fig. 78). The spatial extension of the wave functions of the edge channels is illustrated in Fig. 91 (shaded ellipsoids) for various values of the Fermi level between the $n=3$ and $n=4$ bulk Landau levels. As the Fermi level approaches the $n=3$ bulk Landau level, the corresponding edge channel penetrates into the bulk, so the overlap with the wave functions of lowerindex edge channels decreases. This would explain the decoupling of the $n=3$ and $n=1,2$ edge channels.

These experiments thus point the way in which the transition from microscopic to macroscopic behavior takes place in the QHE, while they also demonstrate that quite large samples will be required before truly macroscopic behavior sets in.

d. Suppression of the Shubnikov-De Haas Oscillations

Shubnikov-De Haas magnetoresistance oscillations were discussed in Sections 4.c and 10. In weak magnetic fields, where a theoretical description 


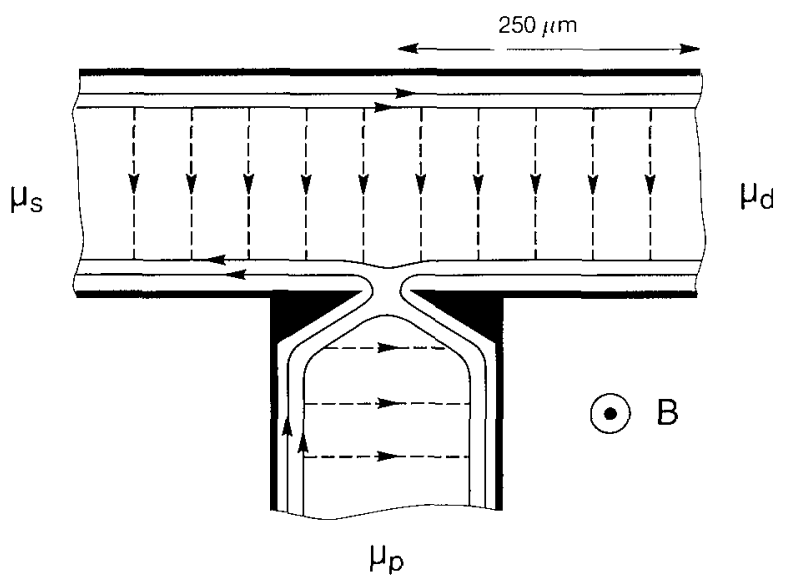

Fig 92 Illustration of the mechanism for the suppression of Shubnikov-De Haas oscillations due to selective detection of edge channels The black area denotes the split-gate point contact in the voltage probe, which is at a distance of $250 \mu \mathrm{m}$ from the drain reservoir Dashed arrows indicate symbolically the selective backscattering in the highest-index edge channel, via states in the highest bulk Landau level that coexist at the Fermı level Taken from $\mathrm{H}$ van Houten $e t$ al, in "Nanostructured Systems" (M A Reed, ed) Academıc, New York 1991

in terms of a local resistivity tensor applies, a satisfactory agreement between theory and experiment is obtained ${ }^{20}$ As we now know, in strong magnetic fields the concept of a local resistivity tensor may break down entirely because of the absence of local equilibrium A theory of the Shubnikov-De Haas effect then has to take into account explicitly the properties of the contacts used for the measurement The resulting anomalies are considered in this subsection

Van Wees et al ${ }^{428}$ found that the amplitude of the high-field ShubnıkovDe Haas oscillations was suppressed if a quantum point contact was used as a voltage probe To discuss this anomalous Shubnikov-De Haas effect, we consider the three-terminal geometry of $F_{1 g} 92$, where a single voltage contact is present on the boundary between source and drain contacts (An alternative two-terminal measurement configuration is also possible, see Ref 428 ) The voltage probe $\mathrm{p}$ is formed by a quantum point contact, while source $\mathrm{s}$ and drain $\mathrm{d}$ are normal ohmic contacts (Note that $t w o$ special contacts were required for the anomalous quantum Hall effect of Section 19c) One straightforwardly finds from Eq (12 12) that the three-terminal resistance $R_{3 \mathrm{t}} \equiv\left(\mu_{\mathrm{p}}-\mu_{\mathrm{d}}\right) / e I$ measured between point contact probe and drain is given by

$$
R_{3 \mathrm{t}}=\frac{h}{2 e^{2}} \frac{T_{\mathrm{s} \rightarrow \mathrm{p}}}{\left(N_{\mathrm{s}}-R_{\mathrm{s}}\right)\left(N_{\mathrm{p}}-R_{\mathrm{p}}\right)-T_{\mathrm{p} \rightarrow \mathrm{s}} T_{\mathrm{s} \rightarrow \mathrm{p}}}
$$




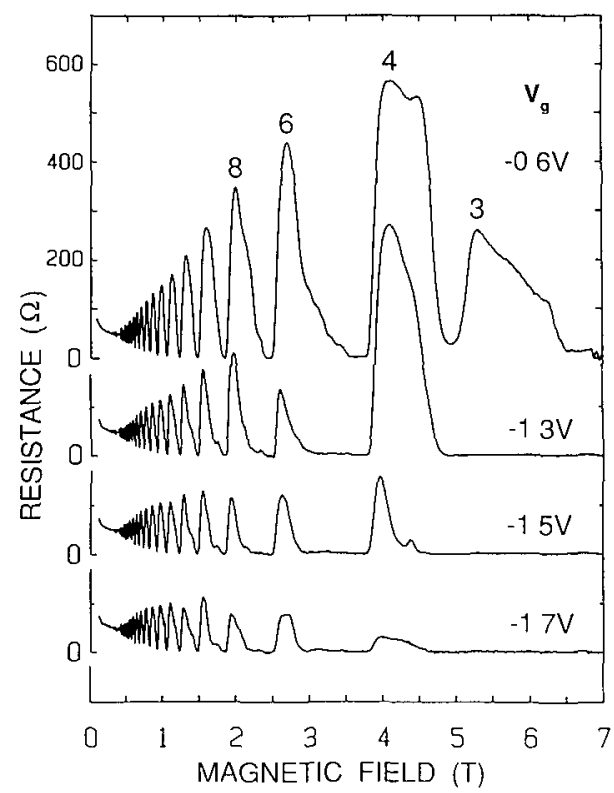

Fig. 93. Measurement of the anomalous Shubnikov-De Haas oscillations in the geometry of Fig. 92. The plotted longitudinal resistance is the voltage drop between contacts $p$ and $d$ divided by the current from $s$ to $d$. At high magnetic fields the oscllations are increasingly suppressed as the point contact in the voltage probe is pinched off by increasing the negative gate voltage. The number of occupied spin-split Landau levels in the bulk is indicated at several of the ShubnikovDe Haas maxima Taken from B. J van Wees et al., Phys. Rev. B. 39, 8066 (1989).

This three-terminal resistance corresponds to a generalized longitudinal resistance if the magnetic field has the direction of Fig. 92. In the absence of backscattering in the $2 \mathrm{DEG}$, one has $T_{\mathrm{s} \rightarrow \mathrm{p}}=0$, so $R_{3 t}$ vanishes, as it should for a longitudinal resistance in a strong magnetic field.

Shubnikov-De Haas oscillations in the longitudinal resistance arise when backscattering leads to $T_{s \rightarrow p} \neq 0$. The resistance reaches a maximum when the Fermi level lies in a bulk Landau level, corresponding to a maximum probability for backscattering (which requires scattering from one edge to the other across the bulk of the sample, as indicated by the dashed lines in Fig. 92). From the preceding discussion of the anomalous quantum Hall effect, we know that the point contact voltage probe in a high magnetic field functions as a selective detector of edge channels with index $n$ less than some value determined by the barrier height in the point contact. If backscattering itself occurs selectively for the channel with the highest index $n=N$, and if the edge channels with $n \leqslant N-1$ do not scatter to that edge channel, then a suppression of the Shubnikov-De Haas oscillations is to be expected when $R_{3 \mathrm{t}}$ 1s measured with a point contact containing a sufficiently high potential barrier. This was indeed observed experimentally, ${ }^{428}$ as shown in Fig. 93. The 
Shubnıkov-De Haas maxımum at $52 \mathrm{~T}$, for example, is found to disappear at gate voltages such that the point contact conductance is equal to, or smaller than $2 e^{2} / h$, which means that the point contact only transmits two spin-split edge channels The number of occupied spin-split Landau levels in the bulk at this magnetic field value is 3 This experiment thus demonstrates that the Shubnıkov-De Haas oscillations result from the highest-index edge channel only, presumably because that edge channel can penetrate into the bulk via states in the bulk Landau level with the same index that coexist at the Fermı level (cf Section 19c) Moreover, it is found that this edge channel does not scatter to the lower-index edge channels over the distance of $250 \mu \mathrm{m}$ from probe $\mathrm{p}$ to drain d, consistent with the experiment of Alphenaar et al ${ }^{429}$

In Section 19 a we discussed how an "1deal" contact at the 2DEG boundary induces a local equilibrium by equipartitioning the outgoing current equally among the edge channels The anomalous Shubnıkov-De Haas effect provides a direct way to study this contact-induced equilibration by means of a second point contact between the point contact voltage probe $\mathrm{p}$ and the current drain d in Fig 92 This experiment was also carried out by van Wees et al, as described in Ref 308 Once again, use was made of the double-split-gate point contact device (Fig 5 b), in this case with a $15-\mu \mathrm{m}$ separation between point contact $\mathrm{p}$ and the second point contact It is found that the Shubnikov-De Haas oscillations in $R_{3 \mathrm{t}}$ are suppressed only if the second point contact has a conductance of $\left(2 e^{2} / h\right)\left(N_{\text {wide }}-1\right)$ or smaller At larger conductances the oscillations in $R_{3 \mathrm{t}}$ return, because this point contact can now couple to the highest-index edge channel and distribute the backscattered electrons over the lower-index edge channels The point contact positioned between contacts $\mathrm{p}$ and $\mathrm{d}$ thus functions as a controllable "edge channel mixer"

The conclusions of the previous paragraph have interesting implications for the Shubnikov-De Haas oscillations in the strong-field regime even if measured with contacts that do not selectively detect certain edge channels only ${ }^{307}$ Consider again the geometry of Fig 92, in the low-gate voltage limit where the point contact voltage probe transmits all edge channels with unit probability (This is the case of an "ideal" contact, cf Section 18 b) To simplify expression (19 12) for the three-terminal longitudinal resistance $R_{3 t}$, we use the fact that the transmission and reflection probabilities $T_{\mathrm{s} \rightarrow \mathrm{p}}, R_{\mathrm{s}}$, and $R_{\mathrm{p}}$ refer to the highest-1ndex edge channel only (with index $n=N$ ), under the assumptions of selective backscattering and absence of scattering to lowerindex edge channels discussed earlier As a consequence, $T_{\mathrm{s} \rightarrow \mathrm{p}}, R_{\mathrm{s}}$, and $R_{\mathrm{p}}$ are each at most equal to 1 , thus, up to corrections smaller by a factor $N^{-1}$, we may put these terms equal to zero in the denominator on the right-hand side of Eq (19 12) In the numerator, the transmission probability $T_{\mathrm{s} \rightarrow \mathrm{p}}$ may be replaced by the backscattering probability $t_{\mathrm{bs}} \leqslant 1$, which 1 the probability 
that the highest-index edge channel injected by the source contact reaches the point contact probe following scattering across the wide 2DEG (dashed lines in Fig. 92). With these simplifications Eq. (19.12) takes the form (assuming spin degeneracy)

$$
R_{3 \mathrm{t}}=\frac{h}{2 e^{2}} \frac{t_{\mathrm{bs}}}{N^{2}} \times\left(1+\operatorname{order} N^{-1}\right)
$$

Only if $t_{\text {bs }} \ll 1$ may the backscattering probability be expected to scale linearly with the separation of the two contacts $p$ and $d$ (between which the voltage drop is measured). If $t_{\mathrm{bs}}$ is not small, then the upper limit $t_{\mathrm{bs}} \leqslant 1$ leads to the prediction of a maximum possible amplitude ${ }^{307}$

$$
R_{\max }=\frac{h}{2 e^{2}} \frac{1}{N^{2}} \times\left(1+\operatorname{order} N^{-1}\right)
$$

of the Shubnikov-De Haas resistance oscillations in a given large magnetic field, independently of the length of the segment over which the voltage drop is measured, provided equilibration does not occur on this segment. Equilibration might result, for example, from the presence of additional contacts between the voltage probes, as discussed before. One easily verifies that the high-field Shubnikov-De Haas oscillations in Fig. 93 at $V_{g}=-0.6 \mathrm{~V}$ (when the point contact is just defined, so that the potential barrier is small) lie well below the upper limit (19.14). For example, the peak around $2 \mathrm{~T}$ corresponds to the case of four occupied spin-degenerate Landau levels, so the theoretical upper limit is $\left(h / 2 e^{2}\right) \times \frac{1}{16} \approx 800 \Omega$, well above the observed peak value of about $350 \Omega$. The prediction of a maximum longitudinal resistance implies that the linear scaling of the amplitude of the Shubnikov-De Haas oscillations with the distance between voltage probes found in the weak-field regime, and expected on the basis of a description in terms of a local resistivity tensor, ${ }^{20}$ breaks down in strong magnetic fields. Anomaious scaling of the Shubnikov-De Haas effect has been observed experimentally $^{457,460,466}$ and has recently also been interpreted ${ }^{40}$ in terms of a nonequilibrium between the edge channels. A quantitative experimental and theoretical investigation of these issues has now been carried out by McEuen et al. $^{477}$

Selective backscattering and the absence of local equilibrium have consequences as well for the two-terminal resistance in strong magnetic fields. ${ }^{307}$ In weak fields one usually observes in two-terminal measurements a superposition of the Shubnikov-De Haas longitudinal resistance oscillations and the quantized Hall resistance. This superposition shows up as a characteristic "overshoot" of the two-terminal resistance as a function of the

\footnotetext{
${ }^{477}$ P. L. McEuen, A. Szafer, C. A. Richter, B. W. Alphenaar, J. K. Jain, and R. N. Sacks, Phys.
}

Rev. Lett. 64, 2062 (1990). 
magnetic field as it increases from one quantized Hall plateau to the next (the plateaux coincide with minıma of the Shubnikov-De Haas oscillations) In the strong-field regime (in the absence of equlibration between source and drain contacts), no such superposition ts to be expected Instead, the twoterminal resistance would increase monotonically from $\left(h / 2 e^{2}\right) N^{-1}$ to $\left(h / 2 e^{2}\right)(N-1)^{-1}$ as the transmission probability from source to drain decreases from $N$ to $N-1$ We are not aware of an experimental test of this prediction

The foregoing analysis assumes that the length $L$ of the conductor is much greater than its width $W$, so edge channels are the only states at the Fermı level that extend from source to drain If $L \ll W$, additional extended states may appear in the bulk of the 2DEG, whenever the Fermı level lies in a bulk Landau level An experiment by Fang et al in this short-channel regime, to which our analysis does not apply, is discussed by Buttıker ${ }^{386}$

\section{Fractional Quantum Hall Effect}

Microscopically, quantization of the Hall conductance $G_{\mathbf{H}}$ in fractional multiples of $e^{2} / h$ is entirely different from quantization in integer multiples While the integer quantum Hall effect ${ }^{8}$ can be explained satisfactorily in terms of the states of noninteracting electrons in a magnetic field (see Section 18), the fractional quantum Hall effect ${ }^{478}$ exists only because of electronelectron interactions ${ }^{479}$ Phenomenologically, however, the two effects are quite similar Several experments on edge channel transport in the integer QHE $^{339340426}$ reviewed in Section 19 have been repeated ${ }^{480481}$ for the fractional QHE with a similar outcome The interpretation of Section 19 in terms of selective population and detection of edge channels cannot be applied in that form to the fractional QHE Edge channels in the integer QHE are defined in one-to-one correspondence to bulk Landau levels (Section $18 \mathrm{~b}$ ) The fractional QHE requires a generalization of the concept of edge channels that allows for independent current channels within the same Landau level Two recent papers have addressed this problem ${ }^{482} 483$ and have obtained different answers The present status of theory and experiment on transport in "fractional" edge channels is reviewed in Section $20 \mathrm{~b}$, preceded by a brief introduction to the fractional QHE

\footnotetext{
${ }^{478}$ D C Tsul, H L Stormer, and A C Gossard, Phys Rev Lett 48, 1559 (1982)

${ }^{479}$ R B Laughlin, Phys Rev Lett 50, 1395 (1983)

${ }^{480}$ A M Chang and J E Cunningham, Sold State Comm 72, 651 (1989), Surf Sct 229, 216 (1990)

${ }^{481} \mathrm{~L}$ P Kouwenhoven, B J van Wees, N C van der Vaart, C J P M Harmans, C E

Timmering, and C T Foxon, Phys Rev Lett 64, 685 (1990), and unpublished

${ }^{482} \mathrm{C}$ W J Beenakker, Phys Rev Lett 64, $216(1990)$

${ }^{483}$ A H MacDonald, Phys Rev Lett 64, 220 (1990)
} 


\section{a Introduction}

Excellent high-level introductions to the fractional QHE in an unbounded 2DEG can be found in Refs 97 and 484 The following is an oversimplificatron of Laughlin's theory ${ }^{479}$ of the effect and is only intended to introduce the reader to some of the concepts that play a role in edge channel transport in the fractional QHE

It is instructive to first consider the motion of two interacting electrons in a strong magnetıc field ${ }^{485}$ The dynamics of the relative coordinate $\mathbf{r}$ decouples from that of the center of mass Semiclassically, $\mathbf{r}$ moves along equipotentials of the Coulomb potential $e^{2} / \varepsilon r$ (this is the guiding center drift discussed in Section $18 \mathrm{~b}$ ) The relative coordinate thus executes a circular motion around the origin, corresponding to the two electrons orbitıng around their center of mass The phase shift acquired on one complete revolution,

$$
\Delta \phi=\frac{e}{\hbar} \oint d \mathbf{l} \cdot \mathbf{A}=\frac{e}{\hbar} B \pi r^{2},
$$

should be an integer multuple of $2 \pi$ so that

$$
r=l_{\mathrm{m}} \sqrt{2 q}, \quad q=1,2,
$$

The interparticle separation in units of the magnetic length $l_{\mathrm{m}} \equiv(\hbar / e B)^{1 / 2}$ is quantized In the field regime where the fractional QHE is observed, only one spin-split Landau level is occupied in general If the electrons have the same spın, the wave function should change sign when two coordinates are interchanged In the case considered here of two electrons, an interchange of the coordinates is equivalent to $\mathbf{r} \rightarrow-\mathbf{r}$ A change of sign is then obtained if the phase shift for one half revolution is an odd multıple of $\pi(1 \mathrm{e}$, for $\Delta \phi$ an odd multiple of $2 \pi$ ) The Pauli principle thus restricts the integer $q$ in $\mathrm{Eq}$ (20 2) to odd values

The interparticle separation of a system of more than two electrons is not quantized Still, one might surmise that the energy at densities $n_{\mathrm{s}} \approx 1 / \pi \bar{r}^{2}$ corresponding to an average separation $\bar{r}$ in accord with Eq (202) would be particularly low This occurs when the Landau level filling factor $v \equiv h n_{\mathrm{s}} / e B$ equals $v \approx 1 / q$ Theoretical work by Laughlın, Haldane, and Halperin 479486487 shows that the energy density $u(v)$ of a uniform 2DEG in a

\footnotetext{
${ }^{484} \mathrm{~T}$ Chakraborty and P Pietılainen, "The Fractional Quantum Hall Effect" Sprınger, Berlın, 1988

${ }^{485}$ R B Laughlın, Phys Rev B 27, 3383 (1983)

${ }^{486}$ F D M Haldane, Phys Rev Lett 51, 605 (1983)

${ }^{487}$ B I Halperın, Phys Rev Lett 52, 1583 (1984)
} 
strong magnetic field has downward cusps at these values of $v$ as well as at other fractions, given generally by

$$
v=p / q
$$

with $p$ and $q$ mutually prime integers and $q$ odd. The cusp in $u$ at integer $v$ is a consequence solely of Landau level quantization, according to

$$
d u / d n_{\mathrm{s}}=\left(\operatorname{Int}[v]+\frac{1}{2}\right) \hbar \omega_{\mathrm{c}} .
$$

Because of the cusp in $u$, the chemical potential $d u / d n_{\mathrm{s}}$ has a discontinuity $\Delta \mu=\hbar \omega_{\mathrm{c}}$ at integer $v$. At these values of the filling factor an infinitesimal increase in electron density costs a finite amount of energy, so the electron gas can be said to be incompressible. The cusp in $u$ at fractional $v$ exists because of the Coulomb interaction. The discontinuity $\Delta \mu$ is now approximately $\Delta \mu \approx e^{2} / \varepsilon l_{\mathrm{m}} \propto \sqrt{B}$, which at a typical field of $6 \mathrm{~T}$ in $\mathrm{GaAs}$ is $10 \mathrm{meV}$, of the same magnitude as the Landau level separation $\hbar \omega_{c} \propto B$.

The incompressibility of the 2DEG at $v=p / q$ implies that a nonzero minimal energy is required to add charge to the system. An important consequence of Laughlin's theory is that charge can be added only in the form of quasiparticle excitations of fractional charge $e^{*}=e / q$. The discontinuity $\Delta \mu$ in the chemical potential equals the energy that it costs to create $p$ pairs of oppositely charged quasiparticles (widely separated from each other), $\Delta \mu=p \times 2 \Delta$ with $\Delta$ the quasiparticle creation energy.

The fractional QHE in a disordered macroscopic sample occurs because the quasiparticles are localized by potential fluctuations in the bulk of the 2DEG. A variation of the filling factor $v=p / q+\delta v$ in an interval around the fractional value changes the density of localized quasiparticles without changing the Hall conductance, which retains the value $G_{\mathrm{H}}=(p / q) e^{2} / h$. The precision of the QHE has been explained by Laughlin ${ }^{488}$ in terms of the quantization of the quasiparticle charge $e^{*}$, which is argued to imply quantization of $G_{\mathbf{H}}$ at integer multiples of $e e^{*} / h$.

\section{b. Fractional Edge Channels}

In a small sample the fractional QHE can occur in the absence of disorder and can show deviations from precise quantization. Moreover, in special geometries ${ }^{481} G_{\mathrm{H}}$ can take on quantized values that are not simply related to $e^{*}$. These observations cannot be easily understood within the conventional description of the fractional QHE, as outlined in the previous subsection. An approach along the lines of the edge channel formulation of the integer QHE (Sections 18 and 19) seems more promising. In Ref. 482 the concept of an edge channel was generalized to the fractional QHE, and a generalized Landauer

${ }^{488}$ R. B. Laughlin, Phys. Rev. B 23, 5632 (1981). 
formula relating the conductance to the transmission probabilities of the edge channels was derived We review this theory and the application to experiments A different edge channel theory by MacDonald ${ }^{483} 1$ discussed toward the end of this subsection

The edge channels for the conductance in the linear transport regime are defined in terms of properties of the equilibrium state of the system If the electrostatic potential energy $V(x, y)$ varies slowly in the $2 \mathrm{DEG}$, then the equilibrium density distribution $n(x, y)$ follows by requiring that the local electrochemical potential $V(\mathbf{r})+d u / d n$ has the same value $\mu$ at each point $\mathbf{r}$ in the 2DEG Here $d u / d n$ is the chemical potential of the unfform 2DEG with density $n(\mathbf{r})$ As discussed in Section 20 a, the internal energy density $u(n)$ of a uniform interacting 2DEG in a strong magnetic field has downward cusps at densities $n=v_{p} B e / h$ corresponding to certain fractional fillıng factors $v_{p}$ As a result, the chemical potential $d u / d n$ has a discontinuty (an energy gap) at $v=v_{p}$, with $d u_{p}^{+} / d n$ and $d u_{p} / d n$ the two limiting values as $v \rightarrow v_{p}$ As noted by Halperın, ${ }^{489}$ when $\mu-V$ lies in the energy gap the fillıng factor is pinned at the value $v_{p}$ The equilibrium electron density is thus given by ${ }^{489}$

$$
\begin{gathered}
n=v_{p} B e / h, \quad \text { if } d u_{p}^{-} / d n<\mu-V<d u_{p}^{+} / d n, \\
d u / d n+V(\mathbf{r})=\mu, \quad \text { otherwise }
\end{gathered}
$$

Note that $V(\mathbf{r})$ itself depends on $n(\mathbf{r})$ and thus has to be determined selfconsistently from Eq (20 5), taking the electrostatic screening in the 2DEG into account We do not need to solve explicitly for $n(\mathbf{r})$, but we can identify the edge channels from the following general considerations ${ }^{482}$

At the edge of the 2DEG, the electron density decreases from its bulk value to zero Eq (20 5) implies that this decrease is stepwise, as illustrated in Fig 94 The requirement on the smoothness of $V$ for the appearance of a welldefined region at the edge in which $v$ is pinned at the fractional value $v_{p}$ is that the change in $V$ within the magnetic length $l_{\mathrm{m}}$ is small compared with the energy gap $d u_{p}^{+} / d n-d u_{p}^{-} / d n$ This ensures that the width of this region is large compared with $l_{\mathrm{m}}$, which is a necessary (and presumably sufficient) condition for the formation of the incompressible state Depending on the smoothness of $V$, one thus obtains a series of steps at $v=v_{p}(p=1,2, \quad, P)$ as one moves from the edge toward the bulk The series terminates in the filling factor $v_{P}=v_{\text {bulk }}$ of the bulk, assuming that in the bulk the chemical potential $\mu-V$ lies in an energy gap The regions of constant $v$ at the edge form bands extending along the wire These incompressible bands [in which the compressibility $\chi \equiv\left(n^{2} d^{2} u / d n^{2}\right)^{-1}=0$ ] alternate with bands in which $\mu-V$ does not lie in an energy gap The latter compressible bands (in which $\chi>0$ ) may be 

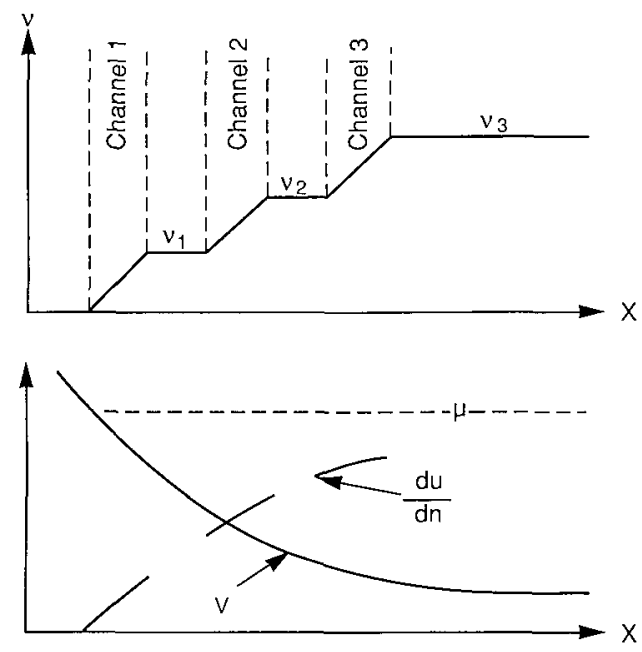

Fig 94 Schematic drawing of the variation in filling factor $v$, electrostatic potential $V$, and chemical potential $d u / d n$, at a smooth boundary in a 2DEG The dashed line in the bottom panel denotes the constant electrochemical potentral $\mu=V+d u / d n$ The dotted intervals indicate a discontinuity (energy gap) in $d u / d n$ and correspond in the top panel to regions of constant fractional filling factor $v_{p}$ that spatially separate the edge channels The width of the edge channel regions shrinks to zero in the integer QHE, since the compressibility $\chi$ of these regions is infinitely large in that case Taken from C W J Beenakker, Phys Rev Lett 64, 216 (1990)

identified as the edge channels of the transport problem, as will be discussed later. To resolve a misunderstanding, ${ }^{490}$ we note that the particular potential and density profile illustrated in Fig. 94 (in which the edge channels have a nonzero width) assumes that the compressibility of the edge channels is not Infinitely large, but the subsequent analysis is independent of this assumption (requiring only that the edge channels are flanked by bands of zero compressibility). Indeed, the analysis is applicable also to the integer QHE, where the edge channels have an infinitely large compressibility and hence an infinitesimally small width (limited only by the magnetic length).

The conductance is calculated by bringing one end of the conductor in contact with a reservoir at a slightly higher electrochemical potential $\mu+\Delta \mu$ without changing $V$ (as in the derivation of the usual Landauer formula; $\mathrm{cf}$. Section 12.b). The resulting change $\Delta n$ in electron density is

$$
\Delta n=\left(\frac{\delta n}{\delta \mu}\right)_{V} \Delta \mu=-\left(\frac{\delta n}{\delta V}\right)_{\mu} \Delta \mu,
$$

where $\delta$ denotes a functional derivative. In the second equality in Eq. (20.6), we used the fact that $n$ is a functional of $\mu-V$, by virtue of Eq. (20.5). In a 
a

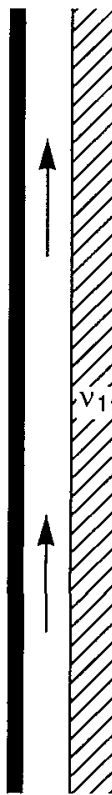

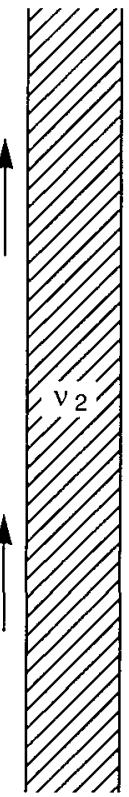
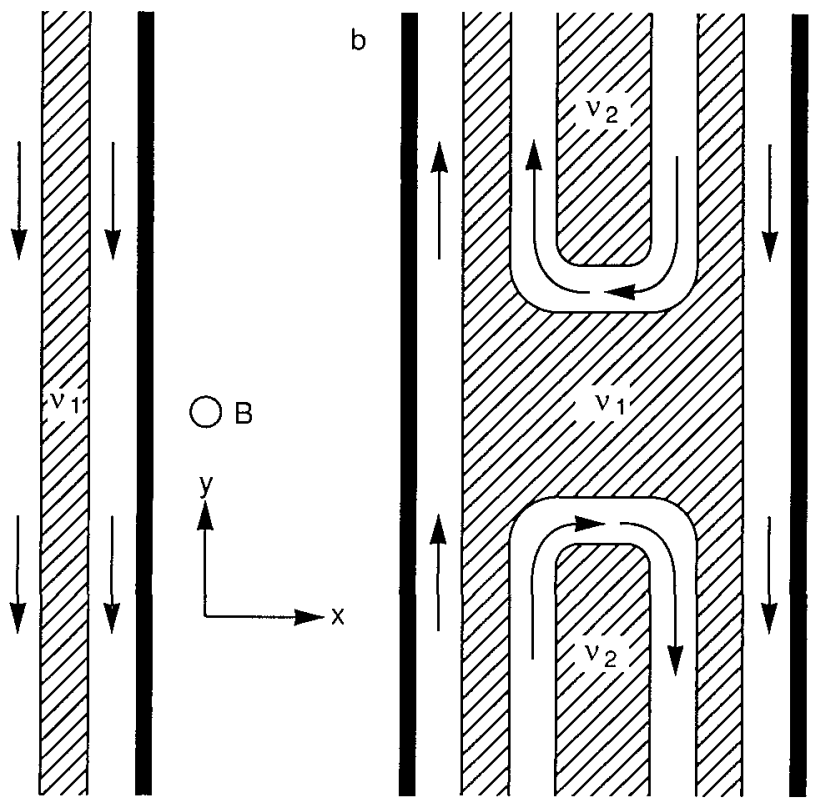

Fig 95 Schematıc drawing of the incompressible bands (hatched) of fractional filling factor $v_{p}$, alternating with the edge channels (arrows indicate the direction of electron motion in each channel) (a) A uniform conductor (b) A conductor contanning a barrier of reduced filling factor Taken from C W J Beenakker, Phys Rev Lett 64, 216 (1990)

strong magnetic field, this excess density moves along equipotentials with the guiding-center-drift velocity $E / B(\mathbf{E} \equiv \partial V / e \partial \mathbf{r}$ being the electric field). The component $v_{\mathrm{drif}}$ of the drift velocity in the $y$-direction (along the conductor) is

$$
v_{\mathrm{drnf}}=\hat{\mathbf{y}} \cdot\left(\mathbf{E} \times \frac{\mathbf{B}}{\boldsymbol{B}^{2}}\right)=-\frac{1}{e B} \frac{\partial \boldsymbol{V}}{\partial x} .
$$

The current density $j=-e \Delta n v_{\text {drift }}$ becomes simply

$$
j=-\frac{e}{h} \Delta \mu \frac{\partial v}{\partial x} .
$$

It follows from Eq. (20.8) that the incompressible bands of constant $v=v_{p}$ do not contribute to $j$. The reservoir injects the current into the compressible bands at one edge of the conductor only (for which the sign of $\partial v / \partial x$ is such that $J$ moves away from the reservoir). The edge channel with index $p=1,2$, $\ldots, P$ is defined as that compressible band that is flanked by incompressible bands at filling factors $v_{p}$ and $v_{p-1}$. The outermost band from the center of the conductor, which is the $p=1$ edge channel, is included by defining formally $v_{0} \equiv 0$. The arrangement of alternating edge channels and compressible bands is 1llustrated in Fig. 95a. Note that different edges may have a different 
series of edge channels at the same magnetic field value, depending on the smoothness of the potential $V$ at the edge (which, as discussed before, determines the incompressible bands that exist at the edge). This is in contrast to the situation in the integer QHE, where a one-to-one correspondence exists between edge channels and bulk Landau levels (Section 18.b). In the fractional QHE an infinite hierarchy of energy gaps exists, in principle, corresponding to an infinite number of possible edge channels, of which only a small number (corresponding to the largest energy gaps) will be realized in practice.

The current $I_{p}=(e / h) \Delta \mu\left(v_{p}-v_{p-1}\right)$ injected into edge channel $p$ by the reservoir follows directly from Eq. (20.8) on integration over $x$. The total current $I$ through the wire is $I=\sum_{p=1}^{p} I_{p} T_{p}$, if a fraction $T_{p}$ of the injected current $I_{p}$ is transmitted to the reservoir at the other end of the wire (the remainder returning via the opposite edge). For the conductance $G \equiv e I / \Delta \mu$, one thus obtains the generalized Landuer formula for a two-terminal conductor, ${ }^{482}$

$$
G=\frac{e^{2}}{h} \sum_{p=1}^{p} T_{p} \Delta v_{p},
$$

which differs from the usual Landauer formula by the presence of the fractional weight factors $\Delta v_{p} \equiv v_{p}-v_{p-1}$. In the integer QHE, $\Delta v_{p}=1$ for all $p$ so that the usual Landauer formula with unit weight factor is recovered.

A multiterminal generalization of Eq. (20.9) for a two-terminal conductor is easily constructed, following Büttiker ${ }^{5}$ (cf. Section 12.b):

$$
\begin{aligned}
I_{\alpha} & =\frac{e}{h} v_{\alpha} \mu_{\alpha}-\frac{e}{h} \sum_{\beta} T_{\alpha \beta} \mu_{\beta}, \\
T_{\alpha \beta} & =\sum_{p=1}^{P_{\beta}} T_{p, \alpha \beta} \Delta v_{p} .
\end{aligned}
$$

Here $I_{\alpha}$ is the current in lead $\alpha$ connected to a reservoir at electrochemical potential $\mu_{\alpha}$ and fractional filling factor $v_{\alpha}$. Equation (20.10b) defines the transmission probability $T_{\alpha \beta}$ from reservoir $\beta$ to reservoir $\alpha$ (or the reflection probability for $\alpha=\beta$ ) in terms of a sum over the generalized edge channels in lead $\beta$. The contribution from each edge channel $p=1,2, \ldots, P_{\beta}$ contains the weight factor $\Delta v_{p} \equiv v_{p}-v_{p-1}$ and the fraction $T_{p, \alpha \beta}$ of the current injected by reservoir $\beta$ into the $p$ th edge channel of lead $\beta$ that reaches reservoir $\alpha$. Apart from the fractional weight factors, the structure of Eq. (20.10) is the same as that of the usual Büttiker formula (12.12).

Applying the generalized Landauer formula (20.9) to the ideal conductor 
in Fig 95a, where $T_{p}=1$ for all $p$, one finds the quantized two-terminal conductance

$$
G=\frac{e^{2}}{h} \sum_{p=1}^{P} \Delta v_{p}=\frac{e^{2}}{h} v_{P}
$$

The four-terminal Hall conductance $G_{H}$ has the same value, because each edge is in local equilibrium In the presence of disorder this edge channel formulation of the fractional QHE is generalized in an analogous way as in the integer QHE by including localized states in the bulk In a smoothly varyıng disorder potentıal, these localized states take the form of circulating edge channels, as in Figs 78 and 79 In this way the filling factor of the bulk can locally deviate from $v_{P}$ without a change in the Hall conductance, leading to the formation of a plateau in the magnetic field dependence of $G_{\mathrm{H}}$ In a narrow channel, localized states are not required for a finite plateau width because the edge channels make it possible for the chemical potential to lie in an energy gap for a fintte-magnetic-field interval The Hall conductance then remains quantized at $v_{P}\left(e^{2} / h\right)$ as long as $\mu-V$ in the bulk lies between $d u_{P}^{+} / d n$ and $d u_{P}^{-} / d n$

We now turn to a discussion of experiments on the fractional QHE in semiconductor nanostructures Timp et al $^{491}$ have measured the fractionally quantized four-terminal Hall conductance $G_{\mathrm{H}}$ in a narrow cross geometry (defined by two sets of split gates) The channel width $W \approx 90 \mathrm{~nm}$ is greater than, but comparable to, the correlation length $l_{\mathrm{m}}$ of the incompressible state in this experiment $\left(l_{\mathrm{m}} \approx 9 \mathrm{~nm}\right.$ at $\left.B=8 \mathrm{~T}\right)$, so one may expect the fractional QHE to be modified by the lateral confinement ${ }^{492}$ Timp et al find, in addition to quantized plateaux near $\frac{1}{3}, \frac{2}{5}$, and $\frac{2}{3} \times e^{2} / h$, a plateau-like feature around $\frac{1}{2} \times e^{2} / h$ This even-denominator fraction is not observed as a Hall plateau in a bulk $2 D E G^{493}$ The plateaux in $G_{H}$ correlate with dips in a fourterminal longitudinal resistance (the bend resistance defined in Section 16)

Consider now a conductor containing a potential barrier The potential barrier corresponds to a region of reduced filling factor $v_{P_{\mathrm{m} n}} \equiv v_{\mathrm{min}}$ separatıng two regions of filling factor $v_{P_{\max }} \equiv v_{\max }$ The arrangement of edge channels and incompressible bands is illustrated in Fig 95b We assume that the potential barrier is sufficiently smooth that scattering between the edge channels at opposite edges can be neglected All transmission probabilities are then elther 0 or $1 T_{p}=1$ for $1 \leqslant p \leqslant P_{\min }$, and $T_{p}=0$ for

${ }^{491} \mathrm{G}$ Timp, R E Behringer, J E Cunningham, and R E Howard, Phys Rev Lett 63, 2268 (1989), G Timp, in Ref 9

${ }^{492}$ S T Chui, Phys Rev Lett 56, 2395 (1986), Phys Rev B 36, 2806 (1987)

${ }^{493}$ H W Jiang, H L Stormer, D C Tsur, L N Pfeiffer, and K W West, Phys Rev B 40, 12013 (1989) 


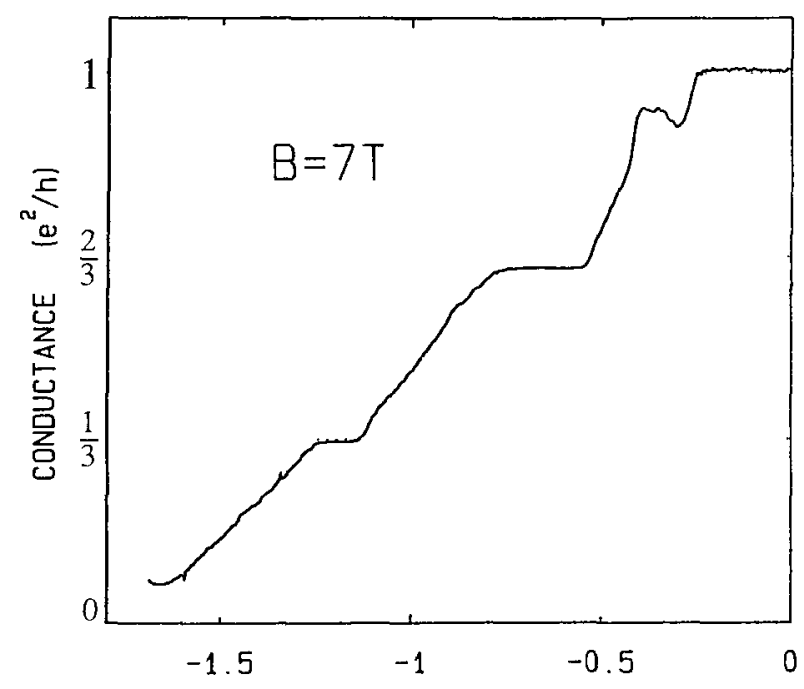

GATE VOLTAGE (V)

FIG. 96. Two-terminal conductance of a constriction containing a potential barrier, as a function of the voltage on the split gate defining the constriction, at a fixed magnetic field of $7 \mathrm{~T}$. The conductance is quantized according to Eq. (20.12). Taken from L. P. Kouwenhoven et al., unpublished.

$P_{\min }<p \leqslant P_{\max }$. Equation (20.9) then tells us that the two-terminal conductance is

$$
G=\left(e^{2} / h\right) v_{\min } .
$$

In Fig. 96 we show experimental data by Kouwenhoven et al. ${ }^{481}$ of the fractionally quantized two-terminal conductance of a constriction containing a potential barrier. The constriction (or point contact) is defined by a split gate on top of a GaAs-AlGaAs heterostructure. The conductance in Fig. 96 is shown for a fixed magnetic field of $7 \mathrm{~T}$ as a function of the gate voltage. Increasing the negative gate voltage increases the barrier height, thereby reducing $G$ below the Hall conductance corresponding to $v_{\max }=1$ in the wide 2DEG. The curve in Fig. 96 shows plateaux corresponding to $v_{\min }=1, \frac{2}{3}$, and $\frac{1}{3}$ in Eq. (20.12). The $\frac{2}{3}$ plateau is not exactly quantized, but is too low by a few percent. The constriction width on this plateau is estimated ${ }^{481}$ at $500 \mathrm{~nm}$, which is a factor of 50 larger than the magnetic length at $B=7 \mathrm{~T}$. It would seem that scattering between fractional edge channels at opposite edges (necessary to reduce the conductance below its quantized value) can only occur via states in the bulk for this large ratio of $W / l_{\mathrm{m}}$.

A four-terminal measurement of the fractional QHE in a conductor 
containing a potential barrier can be analyzed by means of Eq. (20.10), analogously to the case of the integer QHE discussed in Section 19. The fourterminal longitudinal resistance $R_{\mathrm{L}}$ (in the geometry of Fig. 82 ) is given by the analog of Eq. (19.3),

$$
R_{\mathrm{L}}=\frac{h}{e^{2}}\left(\frac{1}{v_{\min }}-\frac{1}{v_{\max }}\right)
$$

provided that either the edge channels transmitted across the barrier have equilibrated with the extra edge channels available outside the barrier region or the voltage contacts are ideal; that is, they have unit transmission probability for all fractional edge channels. Similarly, the four-terminal diagonal resistances $R_{\mathrm{D}}^{ \pm}$defined in Fig. 82 are given by [cf. Eq. (19.5)]

$$
R_{\mathrm{D}}^{+}=\frac{h}{e^{2}} \frac{1}{v_{\min }} ; \quad R_{\mathrm{D}}^{-}=\frac{h}{e^{2}}\left(\frac{2}{v_{\max }}-\frac{1}{v_{\min }}\right)
$$

Chang and Cunningham ${ }^{480}$ have measured $R_{\mathrm{L}}$ and $R_{\mathrm{D}}$ in the fractional QHE, using a $1.5-\mu \mathrm{m}$-wide 2DEG channel with a gate across a segment of the channel (the gate length is also approximately $1.5 \mu \mathrm{m}$ ). Ohmic contacts to the gated and ungated regions allowed $v_{\min }$ and $v_{\max }$ to be determined independently. Equations (20.13) and (20.14) were found to hold to within $0.5 \%$ accuracy. This is illustrated in Fig. 97 for the case that $v_{\max }=1$ and $v_{\min }$ varying from 1 to $2 / 3$ on increasing the negative gate voltage (at a fixed magnetic field of $0.114 \mathrm{~T}$ ). Similar results were obtained ${ }^{480}$ for the case that $v_{\max }=\frac{2}{3}$ and $v_{\min }$ varies from $\frac{2}{3}$ to $\frac{1}{3}$.

Adiabatic transport in the fractional QHE can be studied by the selective population and detection of fractional edge channels, achieved by means of barriers in two closely separated current and voltage contacts (Fig. 98a). The analysis using Eq. (20.10) is completely analogous to the analysis of the experiment in the integer $\mathrm{QHE},{ }^{426}$ discussed in Section 19. Figure $98 \mathrm{~b}$ illustrates the arrangement of edge channels and incompressible bands for the case that the chemical potential lies in an energy gap for the bulk 2DEG (at $v=v_{\text {bulk }}$ ), as well as for the two barriers (at $v_{\mathrm{I}}$ and $v_{\mathrm{v}}$ for the barrier in the current and voltage lead, respectively). Adiabatic transport is assumed over the barrier, as well as from barrier I to barrier V (for the magnetic field direction indicated in Fig. 98). Equation (20.10) for this case reduces to

$$
\begin{aligned}
& I=\frac{e}{h} v_{\mathrm{r}} \mu_{\mathrm{l}}, \\
& 0=\frac{e}{h} v_{\mathrm{v}} \mu_{\mathrm{v}}-\frac{e}{h} \min \left(v_{\mathrm{l}}, v_{\mathrm{v}}\right) \mu_{\mathrm{l}},
\end{aligned}
$$




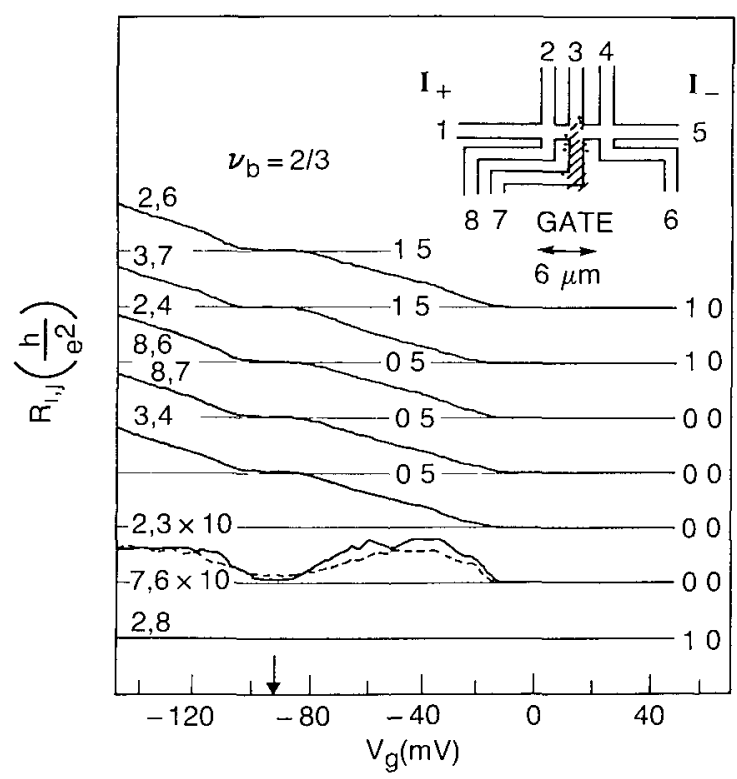

FIG 97 Four-terminal resistances of a $2 \mathrm{DEG}$ channel containıng a potential barrier, as a function of the gate volrage $(B=0114 \mathrm{~T}, T=70 \mathrm{mK})$ The current flows from contact 1 to contact 5 (see inset), the resistance curves are labeled by the contacts $t$ and $J$ between which the voltage 1 measured (The curves for $l, j=2,4$ and 8,6 are identical) The magnetic field points outward This measurement corresponds to the case $v_{\max }=1$ and $v_{\min } \equiv v_{\mathrm{b}}$ varying from 1 at $V_{\mathrm{g}} \geqslant-10 \mathrm{mV}$ to $2 / 3$ at $V_{\mathrm{g}} \approx-90 \mathrm{mV}$ (arrow) The resistances $R_{\mathrm{L}} \equiv R_{24}=R_{86}$ and $R_{\mathrm{D}}^{+} \equiv R_{26}$ are quantized according to Eqs (2013) and (2014), respectively The resistances $R_{37}$ and $R_{2} 8$ are the Hall resistances in the gated and ungated regions, respectively From Eq (2010) one can also derive that $R_{87}=R_{34}=R_{\mathrm{L}}$ and $R_{23}=R_{76}=0$ on the quantized plateaux, as observed experımentally Taken from A M Chang and J E Cunnıngham, Surf $S c$ 229, 216 (1990)

so the Hall conductance $G_{\mathrm{H}}=e I / \mu_{\mathrm{V}}$ becomes

$$
G_{\mathrm{H}}=\frac{e^{2}}{h} \max \left(v_{\mathrm{l}}, v_{\mathrm{V}}\right) \leqslant \frac{e^{2}}{h} v_{\text {bulk }} .
$$

The quantized Hall plateaux are determined by the fractional filling factors of the current and voltage leads, not of the bulk 2DEG. Kouwenhoven et al. ${ }^{481}$ have demonstrated the selective population and detection of fractional edge channels in a device with a $2-\mu \mathrm{m}$ separation of the gates in the current and voltage leads. The gates extended over a length of $40 \mu \mathrm{m}$ along the $2 \mathrm{DEG}$ boundary. In Fig. 99 we reproduce one of the experimental traces of Kouwenhoven et al. The Hall conductance is shown for a fixed magnetic field of $7.8 \mathrm{~T}$ as a function of the gate voltage (all gates being at the same voltage). 

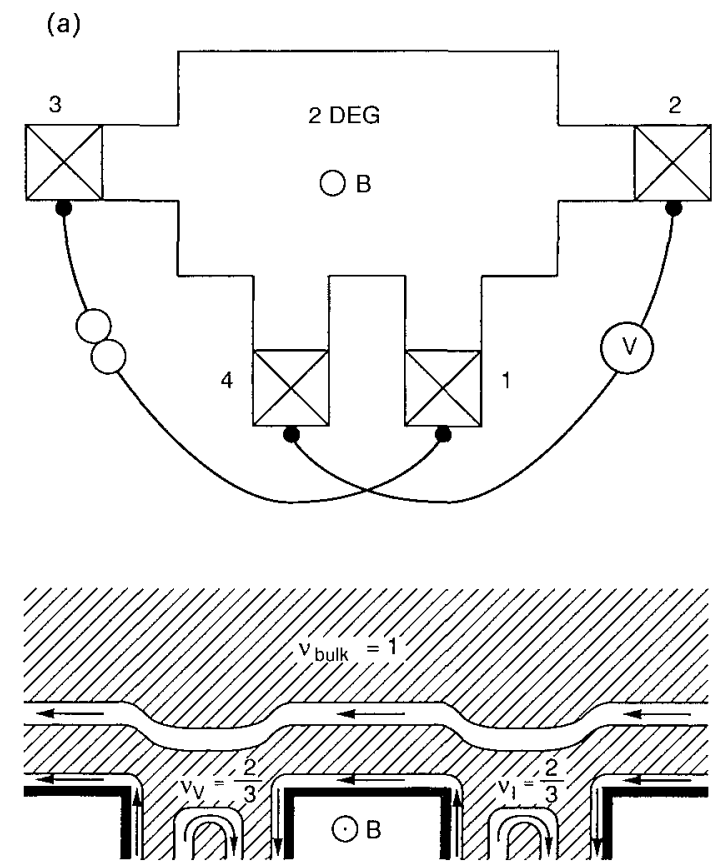

(b)

FIG. 98. (a) Schematic of the experimental geometry of Kouwenhoven et al. ${ }^{481}$ The crossed squares are contacts to the 2DEG. One current lead and one voltage lead contain a barrier (shaded), of which the height can be adjusted by means of a gate (not drawn). The current $I$ flows between contacts 1 and 3; the voltage $V$ is measured between contacts 2 and 4. (b) Arrangement of incompressible bands (hatched) and edge channels near the two barriers. In the absence of scattering between the two fractional edge channels, one would measure a Hall conductance $G_{\mathrm{H}} \equiv I / V$ that is fractionally quantized at $\frac{2}{3} \times e^{2} / h$, although the bulk has unit filling factor. Taken from C. W. J. Beenakker, Phys. Rev. Lett. 64, 216 (1990).

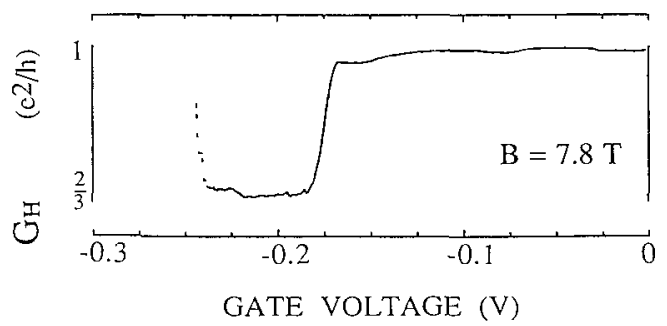

FIG. 99. Anomalously quantized Hall conductance in the geometry of Fig. 98, in accord with Eq. (20.16) $\left(v_{\text {bulk }}=1, v_{I}=v_{\mathrm{v}}\right.$ decreases from 1 to $2 / 3$ as the negative gate voltage is increased). The temperature is $20 \mathrm{mK}$. The rapidly rising part (dotted) is an artifact due to barrier pinch-off. Taken from L. P. Kouwenhoven et al., Phys. Rev. Lett. 64, 685 (1990). 
As the barrier heights in the two leads are increased, the Hall conductance decreases from the bulk value $1 \times e^{2} / h$ to the value $\frac{2}{3} \times e^{2} / h$ determined by the leads, in accord with Eq (2016) A more general formula for $G_{\mathrm{H}}$ valid also in between the quantized plateaux is shown in Ref 481 to be in quantitative agreement with the experiment

MacDonald has, independent of Ref 482, proposed a different generalized Landauer formula for the fractional QHE ${ }^{483}$ The difference with Eq (209) is that the werght factors in MacDonald's formula can take on both positive and negative values (corresponding to electron and hole channels) In the case of local equilibrium at the edge, the sum of weight factors is such that the two formulations give identical results The results differ in the absence of local equilibrium if fractional edge channels are selectively populated and detected For example, MacDonald predicts a negative longitudinal resistance in a conductor at filling factor $v=\frac{2}{3}$ containing a segment at $v=1$ Another implication of Ref 483 is that the two-terminal conductance $G$ of a conductor at $v_{\max }=1$ containing a potential barrier at filling factor $v_{\min } 1$ s reduced to $\frac{1}{3} \times e^{2} / h$ if $v_{\min }=\frac{1}{3}$ [in accord with Eq $\left.(2012)\right]$, but remains at $1 \times e^{2} / h$ if $v_{\min }=2 / 3$ That this is not observed experimentally (cf $F_{11}$ 96) could be due to interedge channel scattering, as argued by MacDonald The experiment by Kouwenhoven et al ${ }^{481}$ (Fig 99), however, is apparently in the adiabatic regime, and was interpreted in Fig 98 in terms of an edge channel of weight $\frac{1}{3}$ at the edge of a conductor at $v=1$ In MacDonald's formulation, the conductor at $v=1$ has only a single edge channel of weight 1 This would need to be reconciled with the experimental observation of quantization of the Hall conductance at $\frac{2}{3} \times e^{2} / h$

We conclude this section by briefly addressing the question What charge does the resistance measure? The fractional quantization of the conductance in the experiments discussed is understood as a consequence of the fractional weight factors in the generalized Landauer formula (209) These weight factors $\Delta v_{p}=v_{p}-v_{p-1}$ are not in general equal to $e^{*} / e$, with $e^{*}$ the fractional charge of the quasiparticle excitations of Laughlın's incompressible state (cf Section 20 a) The reason for the absence of a one-to-one correspondence between $\Delta v_{p}$ and $e^{*}$ is that the edge channels themselves are not incompressible ${ }^{482}$ The transmission probabilities in Eq (209) refer to charged "gapless" excitations of the edge channels, which are not identical to the charge $e^{*}$ excitations above the energy gap in the incompressible bands (the latter charge might be obtained from thermal activation measurements, of Ref

${ }^{494} \mathrm{R}$ G Clark, J R Mallett, S R Haynes, J J Harris and C T Foxon, Phys Rev Lett 60, 1747 (1988)

${ }^{495}$ S A Kivelson and V L Pokrovsky, Phys Rev B 40, 1373 (1989)

${ }^{496} \mathrm{~J}$ A Simmons, H P We1, L W Engel, D C Tsui, and M Shayegan, Phys Rev Lett 63, 1731 (1989) 
494) It is an interesting and (to date) unsolved problem to determine the charge of the edge channel excitations Kivelson and Pokrovsky ${ }^{495}$ have suggested performing tunnelıng experıments in the fractıonal QHE regıme for such a purpose, by using the charge dependence of the magnetic length $(\hbar / e B)^{1 / 2}$ (which determines the penetration of the wave function in a tunnel barrier and, hence, the transmission probability through the barrier) Alternatively, one could use the h/e periodicity of the Aharanov-Bohm magnetoresistance oscillations as a measure of the edge channel charge Simmons et $a l^{496}$ find that the characteristic field scale of quasiperiodic resistance fluctuations in a $2-\mu \mathrm{m}$-wide Hall bar increases from $0016 T \pm 30 \%$ near $v=1,2,3,4$ to $005 \mathrm{~T} \pm 30 \%$ near $v=\frac{1}{3}$ This is suggestive of a reduction in charge from $e$ to $e / 3$, but not conclusive since the area for the AharonovBohm effect is not well defined in a Hall bar (cf Section 21)

\section{Aharonov-Bohm Effect in Strong Magnetic Fields}

As mentioned briefly in Section 8, the Aharonov-Bohm oscillations in the magnetoresistance of a ring are gradually suppressed in strong magnetic fields This suppression provides additional support for edge channel transport in the quantum Hall effect regume (Section 21 a) Entırely new mechanısms for the Aharonov-Bohm effect become operative in strong magnetic fields These mechanisms, resonant tunneling and resonant reflection of edge channels, do not require a ring geometry Theory and experıments on Aharonov--Bohm oscillations in singly connected geometries are the subject of Section $20 \mathrm{~b}$

\section{a Suppression of the Aharonov-Bohm Effect in a Ring}

In Section 8 we have seen how the quantum interference of clockwise and counterclockwise trajectories in a ring in the diffusive transport regime leads to magnetoresistance oscillations with two different periodicities the fundamental Aharonov-Bohm effect with $\Delta B=(h / e) S{ }^{1}$ periodicity, and the harmonic with $\Delta B=(h / 2 e) S^{-1}$ periodicity, where $S$ is the area of the ring In arrays of rings only the $h / 2 e$ effect is observable, since the $h / e$ effect has a sample specific phase and is averaged to zero In experiments by Timp et al ${ }^{69}$ and by Ford et $\mathrm{al}^{74}$ on single rings in the 2DEG of high-mobility GaAsAlGaAs heterostructures, the $h / e$ effect was found predominantly The amplitude of these oscillations is strongly reduced ${ }^{69} 74195497$ by a large magnetic field (cf the magnetoresistance traces shown in Fig 26) This suppression was found to occur for fields such that $2 l_{\text {cycl }}<W$, where $W$ is the width of the arms of the ring The reason is that in strong magnetic fields the

${ }^{497}$ G Timp, P M Mankıewich, P DeVegvar, R Behrınger, J E Cunningham, R E Howard, H

U Baranger, and J K Jain, Phys Rev B 39, 6227 (1989) 


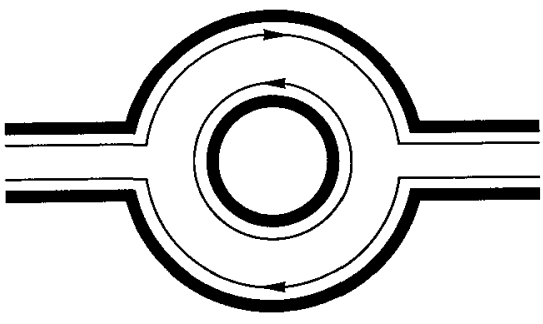

Fig. 100. Illustration of a localized edge channel circulating along the inner perimeter of a ring, and of extended edge channels on the leads and on the outer perimeter. No Aharonov-Bohm magnetoresistance oscillations can occur in the absence of scattering between these two types of edge channels.

states at the Fermi level that can propagate through the ring are edge states at the outer perimeter. These states do not complete a revolution around the ring (see Fig. 100). Scattering between opposite edges is required to complete a revolution, but such backscattering would also lead to a nonzero longitudinal resistance. This argument ${ }^{112,498}$ explains the absence of AharonovBohm oscillations on the quantized Hall plateaux, where the longitudinal resistance is zero. Magnetoresistance oscillations return between the plateaux in the Hall resistance, but at a larger value of $\Delta B$ than in weak fields. Timp et $a l^{497}$ have argued that the Aharonov-Bohm oscillations in a ring in strong magnetic fields are associated with scattering from the outer edge to edge states circulating along the inner perimeter of the ring. The smaller area enclosed by the inner perimeter explains the increase in $\Delta B$.This interpretation is supported by numerical calculations. ${ }^{497}$

\section{b. Aharonov-Bohm Effect in Singly Connected Geometries}

(1) Point Contact. Aharonov-Bohm oscillations in the magnetoresistance of a quantum point contact were discovered by van Loosdrecht et al. ${ }^{292}$ The magnetic field dependence of the two-terminal resistance is shown in Fig. 101, for various gate voltages. The periodic oscillations occur predominantly between quantum Hall plateaux, in a limited range of gate voltages, and only at low temperatures (in Fig. 101, T=50 mK; the effect has disappeared at $1 \mathrm{~K}$ ). The fine structure is very well reproducible if the sample is kept in the cold, but changes after cycling to room temperature. As one can see from the enlargements in Fig. 102, a splitting of the peaks occurs in a range of magnetic fields, presumably as spin splitting becomes resolved. A curious aspect of the effect (which has remained unexplained) is that the oscillations have a much larger amplitude in one field direction than in the other (see Fig. 101), in apparent conflict with the $\pm B$ symmetry of the two-terminal resistance required by the reciprocity relation (12.16) in the absence of magnetic impurities. Other devices of the same design did not show oscillations of welldefined periodicity and had a two-terminal resistance that was approximately $\pm B$ symmetric. 


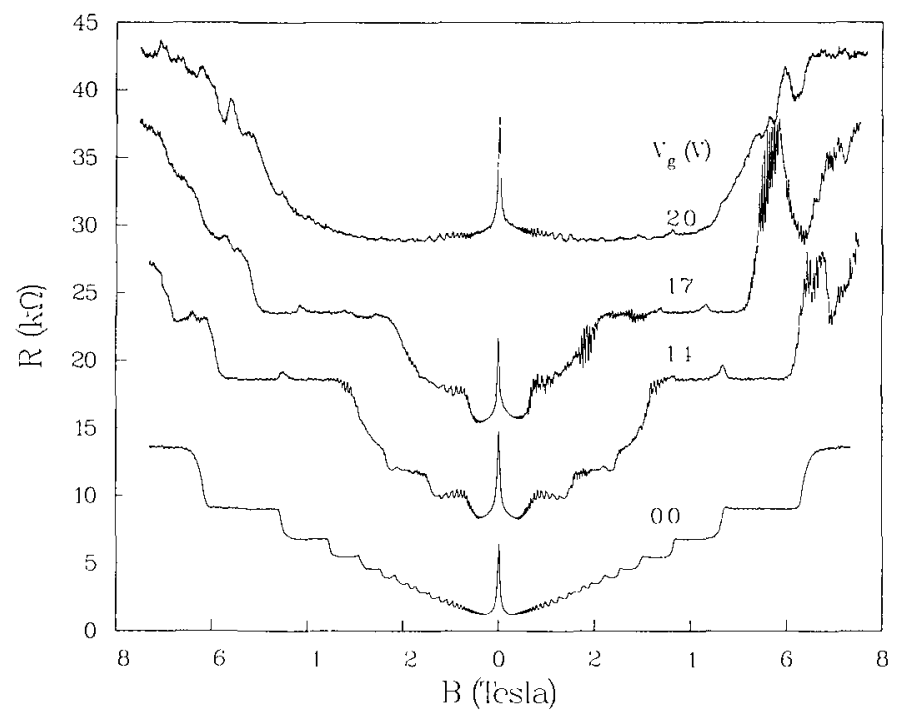

FIG. 101. Two-terminal magnetoresistance of a point contact for a series of gate voltages at $T=50 \mathrm{mK}$, showing oscillations that are periodic in $B$ between the quantum Hall plateaux. The second, third, and fourth curves from the bottom have offsets of, respectively, 5, 10, and $15 \mathrm{k} \Omega$. The rapid oscillations below $1 \mathrm{~T}$ are Shubnikov-De Haas oscillations periodic in $1 / B$, originating from the wide $2 \mathrm{DEG}$ regions. The sharp peak around $B=0 \mathrm{~T}$ originates from the ohmic contacts. Taken from P. H. M. van Loosdrecht et al., Phys. Rev. B 38, 10162 (1988).

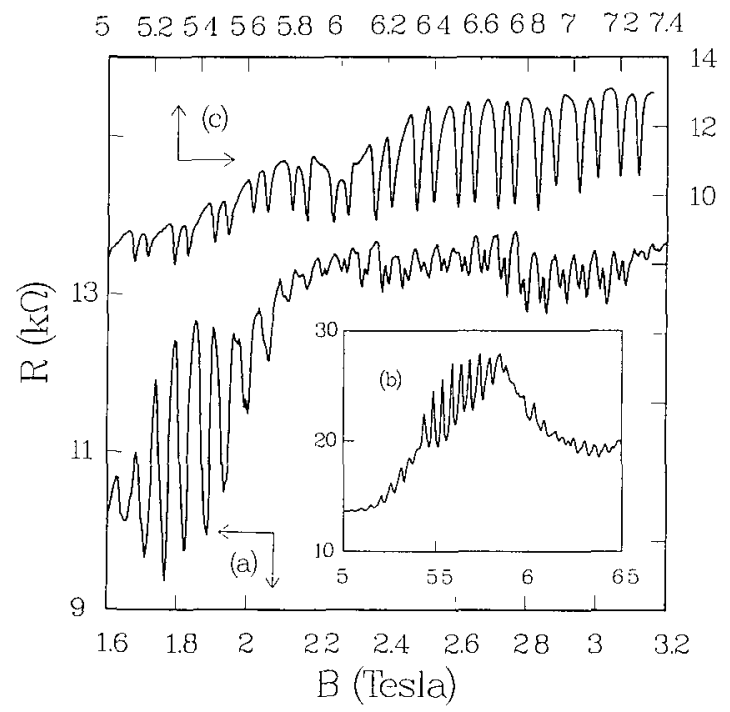

FIG. 102. Curves a and $\mathrm{b}$ are close-ups of the curve for $V_{\mathrm{g}}=-1.7 \mathrm{~V}$ in Fig. 101. Curve $\mathrm{c}$ is a separate measurement on the same device (note the different field scale due to a change in electron density in the constriction). Taken from P. H. M. van Loosdrecht et al., Phys. Rev. B 38, 10162 (1988). 


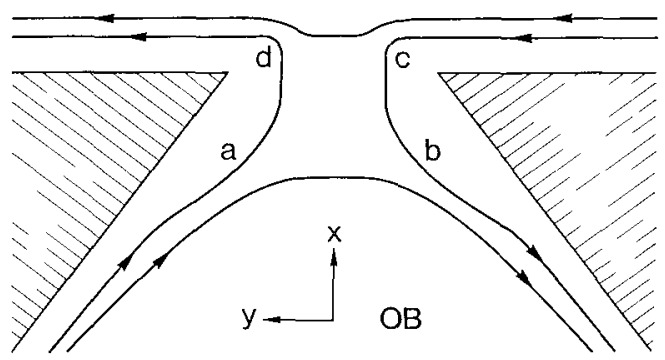

FIG 103 Equipotentials at the guiding center energy in the saddle-shaped potential created by a split gate (shaded) Aharonov-Bohm oscillations in the point contact magnetoresistance result from the interference of tunneling paths $a b$ and $a d c b$ Tunneling from $a$ to $b$ may be assisted by an impurity at the entrance of the constriction Taken from P H M van Loosdrecht et al, Phys Rev B 38, 10162 (1988)

Figure 103 illustrates the tunneling mechanism for the periodic magnetoresistance oscillations as it was originally proposed ${ }^{292}$ to explain the observations Because of the presence of a barrier in the point contact, the electrostatic potential has a saddle form Equipotentials at the guiding center energy (18 1) are drawn schematically in Fig 103 (arrows indicate the direction of motion along the equipotential) An electron that enters the constriction at $a$ can be reflected back into the broad region by tunneling to the opposite edge, either at the potential step at the entrance of the constriction (from $a$ to $b$ ) or at its exit (from $d$ to $c$ ) These two tunneling paths acquire an Aharonov-Bohm phase difference ${ }^{499}$ of $e B S / \hbar$ (were $S$ is the enclosed area $a b c d$ ), leading to periodic magnetoresistance oscillations (Note that the periodicity $\Delta B$ may differ ${ }^{438}{ }^{500}$ somewhat from the usual expression $\Delta B=h / e S$, since $S$ itself is $B$-dependent due to the $B$-dependence of the guiding center energy) This mechanism shows how an Aharonov-Bohm effect is possible in principle in a singly connected geometry The point contact behaves as if it were multiply connected, by virtue of the spatial separation of edge channels moving in opposite directions (Related mechan1sms, based on circulating edge currents, have been considered for Aharonov-Bohm effects in small conductors $473474501-503$ ) The oscillations

${ }^{499} \mathrm{~J}$ K Jain and S Kivelson, Phys Rev B 37, 4111 (1988)

${ }^{500}$ B J van Wees, L P Kouwenhoven, C J P M Harmans, J G Williamson, C E T

Timmering, M E I Broekaart, C T Foxon, and J J Harris, Phys Rev Lett 62, 2523 (1989)

${ }^{501} \mathrm{E}$ N Bogachek and G A Gogadze, Zh Eksp Teor Flz 63, 1839 (1972) [Sov Phys JETP 36, 973 (1973)]

${ }^{502} \mathrm{~N}$ B Brandt, D V Gitsu, A A Nikolaevna, and Ya G Ponomarev, Zh Eksp Teor Fiz 72, 2332 (1977) [Sov Phys JETP 45, 1226 (1977)], N B Brandt, D B Gitsu, V A Dolma, and Ya G Ponomarev, Zh Eksp Teor Flz 92, 913 (1987) [Sov Phys JETP 65, 515 (1987)] ${ }^{503}$ Y Isawa, Surf $S c t$ 170, 38 (1986) 


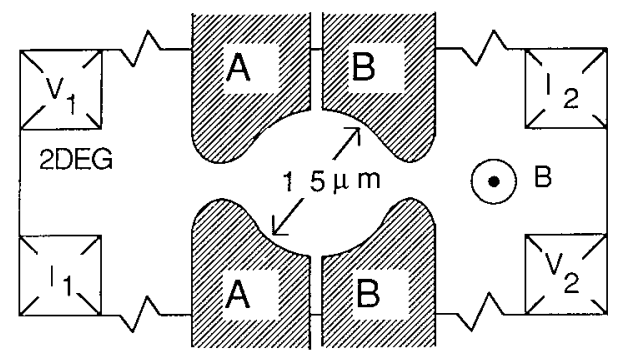

Fig 104 Cavity (of $15 \mu \mathrm{m}$ drameter) defined by a double set of split gates A and B For large negative gate voltages the $2 \mathrm{DEG}$ region under the narrow gap between gates $\mathrm{A}$ and $\mathrm{B}$ is fully depleted, while transmission remains possible over the potential barrier in the wider openings at the left and right of the cavity Taken from B J van Wees et al, Phys Rev Lett 62, 2523 (1989)

periodic in $B$ are only observed at large magnetic fields (above about $1 \mathrm{~T}$, the oscillations at lower fields are Shubnikov-De Haas oscillations periodic in $1 / B$, due to the series resistance of the wide $2 \mathrm{DEG}$ regions) At low magnetic fields the spatial separation of edge channels responsible for the AharanovBohm effect is not yet effective The spatial separation can also be destroyed by a large negative gate voltage (top curve in Fig 101), when the width of the point contact becomes so small that the wave functions of edge states at opposite edges overlap

Although the mechanism illustrated in Fig 103 is attractive because it is an intrinsic consequence of the point contact geometry, the observed welldefined periodicity of the magnetoresistance oscillations requires that the potential induced by the split gate varies rapidly over a short distance (in order to have a well-defined area $S$ ) A smooth saddle potential seems more realistic Moreover, one would expect the periodicity to vary more strongly with gate voltage than the small $10 \%$ variation observed experimentally as $V_{\mathrm{g}}$ 1s changed from -14 to $-17 \mathrm{~V}$ Glazman and Jonson ${ }^{438}$ have proposed that one of the two tunneling processes (from $a$ to $b$ in Fig 103) is mediated by an impurity outside but close to the constriction The combination of impurity and point contact introduces a well-defined area even for a smooth saddle potential, which moreover will not be strongly gate-voltagedependent Such an impurity-assisted Aharonov-Bohm effect in a quantum point contact has been reported by Wharam et al ${ }^{504}$ In order to study the Aharonov-Bohm effect due to interedge channel tunneling under more controlled conditions, a double-point contact device is required, as discussed below

(2) Cavity. Van Wees et al ${ }^{500}$ performed magnetoresıstance experıments in a geometry shown schematically in Fig 104 A cavity with two opposite point

${ }^{504}$ D A Wharam M Pepper, R Newbury, H Ahmed, D G Hasko, D C Peacock, J E F

Frost, D A Ritchie, and G A C Jones, J Phys Condens Matter 1, 3369 (1989) 


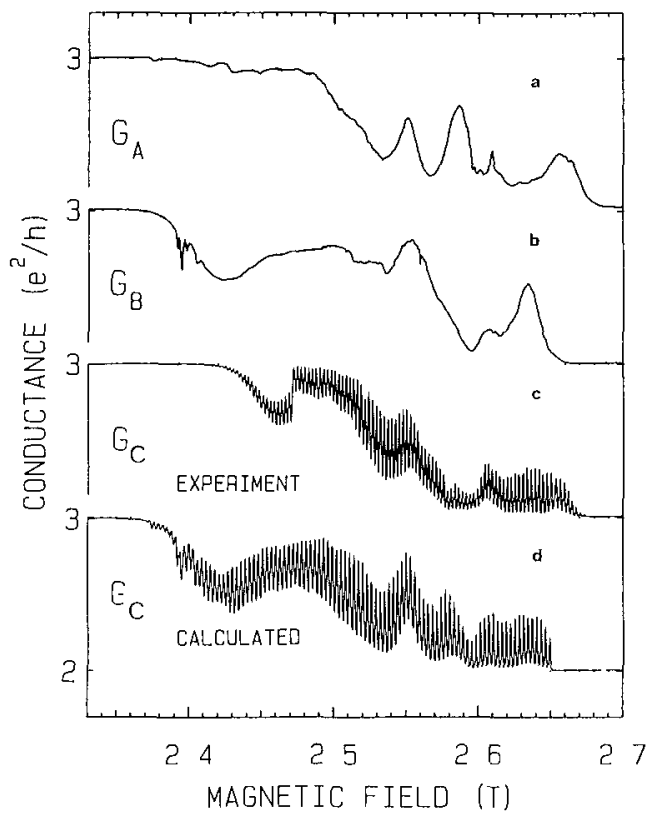

Fig. 105. Magnetoconductance experiments on the device of Fig. 104 at $6 \mathrm{mK}$, for a fixed gate voltage of $-0.35 \mathrm{~V}$. (a) Conductance of point contact A, measured with gate B grounded. (b) Conductance of point contact B (gate A grounded). (c) Measured conductance of the entire cavity. (d) Calculated conductance of the cavity, obtained from Eqs. (21.1) and (21.2) with the measured $G_{A}$ and $G_{\mathrm{B}}$ as input. Taken from B. J. van Wees et al., Phys. Rev. Lett. 62, 2523 (1989).

contact openings is defined in the 2DEG by split gates. The diameter of the cavity is approximately $1.5 \mu \mathrm{m}$. The conductances $G_{\mathrm{A}}$ and $G_{\mathrm{B}}$ of the two point contacts $\mathrm{A}$ and $\mathrm{B}$ can be measured independently (by grounding one set of gates), with the results plotted in Fig. 105a, b (for $V_{\mathrm{g}}=-0.35 \mathrm{~V}$ on either gate A or B). The conductance $G_{\mathrm{C}}$ of the cavity (for $V_{\mathrm{g}}=-0.35 \mathrm{~V}$ on both the split gates) is plotted in Fig. 105c. A long series of periodic oscillations is observed between two quantum Hall plateaux. Similar series of oscillations (but with a different periodicity) have been observed between other quantum Hall plateaux. The oscillations are suppressed on the plateaux themselves. The amplitude of the oscillations is comparable to that observed in the experiment on a single point contact ${ }^{292}$ (discussed before), but the period is much smaller (consistent with a larger effective area in the double-point contact device), and no splitting of the peaks is observed (presumably due to a fully resolved spin degeneracy). No gross $\pm B$ asymmetries were found in the present experiment, although an accurate test of the symmetry on field reversal was not possible because of difficulties with the reproducibility. The 
a

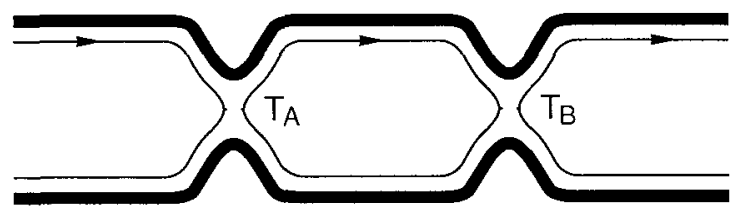

b

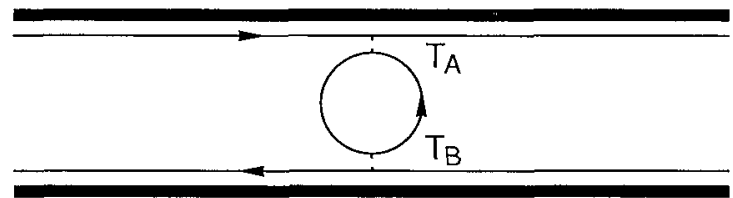

Fig 106 Illustration of mechanisms leading to Aharonov-Bohm oscllations in singly connected geometries (a) Cavity containing a circulating edge state Tunnelıng through the left and right barriers (as indicated by dashed lines) occurs with transmission probabilities $T_{A}$ and $T_{B}$ On increasing the magnetic field, resonant tunneling through the cavity occurs periodically each tume the flux $\Phi$ enclosed by the circulating edge state increases by one flux quantum $h / e$ (b) A circulating edge state bound on a local potential maximum causes resonant backscattering, rather than resonant transmission

oscllations are quite fragile, disappearing when the temperature is raised above $200 \mathrm{mK}$ or when the voltage across the device exceeds $40 \mu \mathrm{V}$ (the data in Fig. 105 were taken at $6 \mathrm{mK}$ and $6 \mu \mathrm{V}$ ). The experimental data are well described by resonant transmission through a circulating edge state in the cavity, ${ }^{500}$ as illustrated in Fig. 106a and described in detail later. AharonovBohm oscillations due to resonant transmission through a similar structure have been reported by Brown et al. ${ }^{505}$ and analyzed theoretically by Yosephin and Kaveh. ${ }^{506}$

(3) Resonant Transmission and Reflection of Edge Channels. The electrostatıc potential in a point contact has a saddle shape (cf. Fig. 103), due to the combination of the lateral confinement and the potential barrier. The height of the barrier can be adjusted by means of the gate voltage. An edge state with a guiding center energy below the barrier height is a bound state in the cavity formed by two opposite point contacts, as is illustrated in Fig. 106a.

${ }^{505}$ R J Brown, C G Smith, M Pepper, M J Kelly, R Newbury, H Ahmed, D G Hasko, J E

F Frost, D C Peacock, D A Ritchie, and G A C Jones, J Phys Condens Matter 1, 6291 (1989)

${ }^{506}$ Y Yosephın and M Kaveh, J Phys Condens Matter 1, 10207 (1989) 
Tunneling of edge channels through the cavity via this bound state occurs with transmission probability $T_{\mathrm{AB}}$, which for a single edge channel is given by ${ }^{474,498}$

$$
T_{\mathrm{AB}}=\left|\frac{t_{\mathrm{A}} t_{\mathrm{B}}}{1-r_{\mathrm{A}} r_{\mathrm{B}} \exp (\mathrm{i} \Phi e / \hbar)}\right|^{2}=\frac{T_{\mathrm{A}} T_{\mathrm{B}}}{1+R_{\mathrm{A}} R_{\mathrm{B}}-2\left(R_{\mathrm{A}} R_{\mathrm{B}}\right)^{1 / 2} \cos \left(\phi_{0}+\Phi e / \hbar\right)} .
$$

Here $t_{\mathrm{A}}$ and $r_{\mathrm{A}}$ are the transmission and reflection probability amplitudes through point contact $\mathrm{A}, T_{\mathrm{A}} \equiv\left|t_{\mathrm{A}}\right|^{2}$, and $R_{\mathrm{A}} \equiv\left|r_{\mathrm{A}}\right|^{2}=1-T_{\mathrm{A}}$ are the transmission and reflection probabilities, and $t_{\mathrm{B}}, r_{\mathrm{B}}, T_{\mathrm{B}}, R_{\mathrm{B}}$ denote the corresponding quantities for point contact B. In Eq. (21.1) the phase acquired by the electron on one revolution around the cavity is the sum of the phase $\phi_{0}$ from the reflection probability amplitudes (which can be assumed to be only weakly $B$-dependent) and of the Aharonov-Bohm phase $\Phi \equiv B S$, which varies rapidly with $B(\Phi$ is the flux through the area $S$ enclosed by the equipotential along which the circulating edge state is extended). Resonant transmission occurs periodically with $B$, whenever $\phi_{0}+\Phi e / \hbar$ is a multiple of $2 \pi$. In the weak coupling limit $\left(T_{A}, T_{B} \ll 1\right)$, Eq. (21.1) is equivalent to the Breit-Wigner resonant tunneling formula (17.1). This equivalence has been discussed by Büttiker, ${ }^{386}$ who has also pointed out that the Breit-Wigner formula is more generally applicable to the case that several edge channels tunnel through the cavity via the same bound state.

In the case that only a single (spin-split) edge channel is occupied in the $2 \mathrm{DEG}$, the conductance $G_{\mathrm{C}}=\left(e^{2} / h\right) T_{\mathrm{AB}}$ of the cavity follows directly from Eq. (21.1). The transmission and reflection probabilities can be determined independently from the individual point contact conductances $G_{\mathrm{A}}=\left(e^{2} / h\right) T_{\mathrm{A}}$ (and similarly for $G_{\mathrm{B}}$ ), at least if one may assume that the presence of the cavity has no effect on $T_{\mathrm{A}}$ and $T_{\mathrm{B}}$ itself (but only on the total transmission probability $T_{\mathrm{AB}}$ ). If $N>1$ spin-split edge channels are occupied and the $N-1$ lowest-index edge channels are fully transmitted, one can write

$$
G_{\mathrm{C}}=\frac{e^{2}}{h}\left(N-1+T_{\mathrm{AB}}\right), \quad G_{\mathrm{A}}=\frac{e^{2}}{h}\left(N-1+T_{\mathrm{A}}\right), \quad G_{\mathrm{B}}=\frac{e^{2}}{h}\left(N-1+T_{\mathrm{B}}\right) .
$$

Van Wees et al. ${ }^{500}$ have compared this simple model with their experimental data, as shown in Fig. 105. The trace in Fig. 105d has been calculated from Eqs. (21.1) and (21.2) by using the individual point contact conductances in Fig. 105a, b as input for $T_{\mathrm{A}}$ and $T_{\mathrm{B}}$. The flux $\Phi$ has been adjusted to the experimental periodicity of $3 \mathrm{mT}$, and the phase $\phi_{0}$ in Eq. (21.1) has been ignored (since that would only amount to a phase shift of the oscillations). Energy averaging due to the finite temperature and voltage has been taken 
into account in the calculation. The agreement with experimental trace (Fig. $105 \mathrm{c})$ is quite satisfactory.

Resonant reflection of an edge channel can occur in addition to the resonant transmission already considered. Aharonov-Bohm oscillations due to interference of the reflections at the entrance and exit of a point contact, illustrated in Fig. 103, are one example of resonant reflection. ${ }^{292}$ Jain $^{498}$ has considered resonant reflection via a localized state circulating around a potential maximum, as in Fig. 106b. Such a maximum may result naturally from a repulsive scatterer or artificially in a ring geometry (cf. Fig. 100). Tunneling of an edge state at each of the channel boundaries through the localized state occurs with probabilities $T_{\mathrm{A}}$ and $T_{\mathrm{B}}$. The reflection probability of the edge channel is still given by $T_{\mathrm{AB}}$ in Eq. (20.1), but the channel conductance $G_{\mathrm{C}}$ is now a decreasing function of $T_{\mathrm{AB}}$, according to

$$
G_{\mathrm{C}}=\frac{e^{2}}{h}\left(N-T_{\mathrm{AB}}\right)
$$

Quasi-periodic magnetoresistance oscillations have been observed in narrow channels by several groups. ${ }^{70,496,507}$ These may occur by resonant reflection via one or more localized states in the channel, as in Fig. 106b.

\section{Magnetically Induced Band Structure}

The one-dimensional nature of edge channel transport has recently been exploited in an innovative way by Kouwenhoven et al. ${ }^{250}$ to realize a onedimensional superlattice exhibiting band structure in strong magnetic fields. The one-dimensionality results because only the highest-index edge channel (with the smallest guiding center energy) has an appreciable backscattering probability. The $N-1$ lower-index edge channels propagate adiabatically, with approximately unit transmission probability. One-dimensionality in zero magnetic fields cannot be achieved with present techniques. That is one important reason why the zero-field superlattice experiments described in Section 11 could not provide conclusive evidence for a bandstructure effect. The work by Kouwenhoven et al. ${ }^{250}$ is reviewed in Section 22.a. The magnetically induced band structure differs in an interesting way from the zero-field band structure familiar from solid-state textbooks, as we show in Section 22.b.

\section{a. Magnetotransport through a One-Dimensional Superlattice}

The device studied by Kouwenhoven et al. ${ }^{250}$ is shown in the inset of Fig. 107. A narrow channel is defined in the 2DEG of a GaAs-AlGaAs

${ }^{507}$ R. Mottahedeh, M. Pepper, R. Newbury, J. A. A. J. Perenboom, and K.-F. Berggren, Solid State Comm. 72, 1065 (1989). 


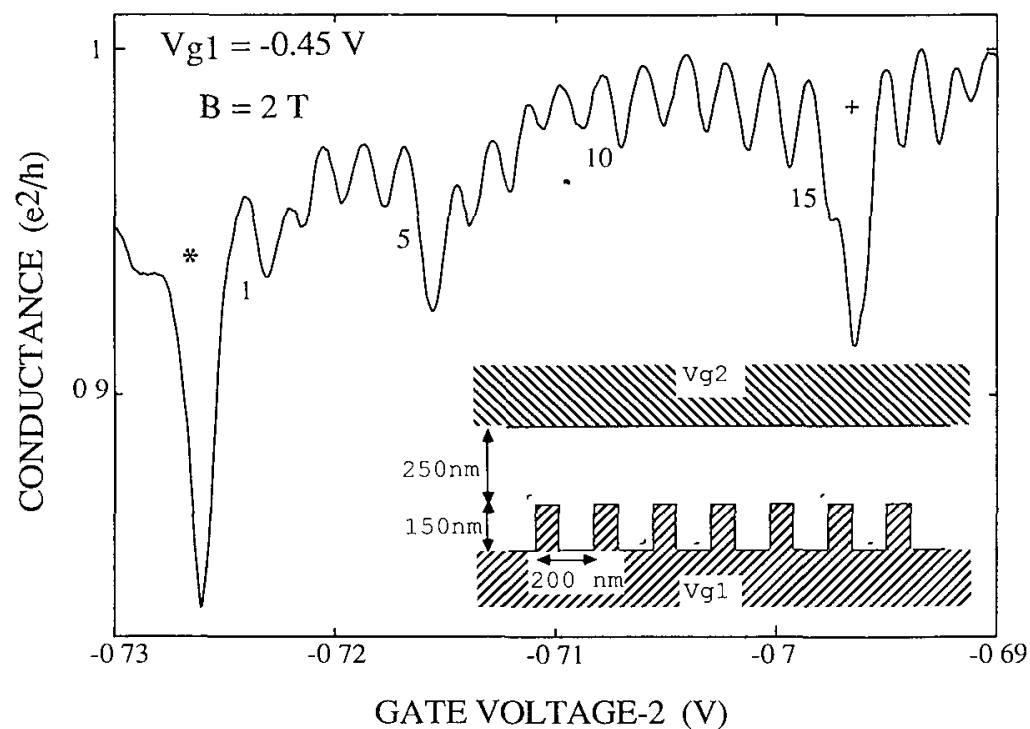

FIg 107 Inset Corrugated gate used to define a narrow channel with a one-dimensional periodic potentual (the total number of barriers 1s 16, corresponding to 15 unit cells) Plotted is the conductance in a magnetic field of $2 \mathrm{~T}$ as a function of the voltage on the smooth gate at $10 \mathrm{mK}$ The deep conductance minıma (marked by + and *) are attributed to minigaps, and the 15 enclosed maxima to discrete states in the miniband Taken from $\mathrm{L} P$ Kouwenhoven et al, Phys Rev Lett 65, 361 (1990)

heterostructure by two opposite gates. One of the gates is corrugated with period $a=200 \mathrm{~nm}$, to introduce a periodic modulation of the confining potential. At large negative gate voltages the channel consists of 15 cavities [as in Section 21.b(2)] coupled in series. The conductance of the channel was measured at $10 \mathrm{mK}$ in a fixed magnetic field of $2 \mathrm{~T}$, as a function of the voltage on the gate that defines the smooth channel boundary. The results, reproduced in Fig. 107, show two pronounced conductance dips (of magnitude $\left.0.1 e^{2} / h\right)$, with 15 oscillations in between of considerably smaller amplitude. The two deep and widely spaced dips are attributed to minigaps, the more rapid oscillations to discrete states in the miniband.

This interpretation is supported in Ref. 250 by a calculation of the transmission probability amplitude $t_{n}$ through $n$ cavities in series, given by the recursion formula

$$
t_{n}=\frac{t t_{n-1}}{1-r r_{n-1} \exp (\mathrm{i} \phi)} .
$$

Here $t$ and $r$ are transmission and reflection probability amplitudes of the barrier separatıng two cavities (all cavitities are assumed to be identical), and 


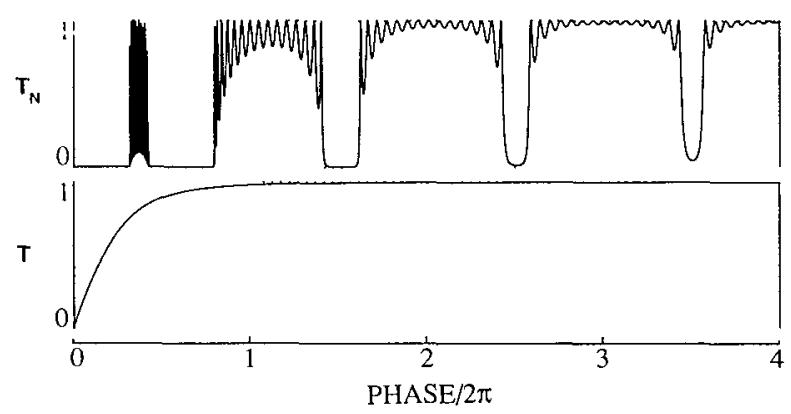

FIG. 108. Top: Calculated transmission probability $T_{\mathrm{N}}$ of an edge channel through a periodic potential of $N=15$ periods as a function of the Aharonov-Bohm phase $e B S / \hbar$ (with $S$ the area of one unit cell). The transmission probability through a single barrier is varied as shown in the bottom panel. Taken from L. P. Kouwenhoven et al., Phys. Rev. Lett. 65, 361 (1990).

$\phi=e B S / \hbar$ is the Aharonov-Bohm phase for a circulating edge state enclosing area $S$. Equation (22.1) is a generalization of Eq. (21.1) for a single cavity. The dependence on $\phi$ of $T_{n}=\left|t_{n}\right|^{2}$ shown in Fig. 108 is indeed qualitatively similar to the experiment. Deep minima in the transmission probability occur with periodicity $\Delta \phi=2 \pi$. Experimentally (where $S$ is varied via the gate voltage at constant $B$ ) this would correspond to oscillations with periodicity $\Delta S=h / e B$ of Aharonov-Bohm oscillations in a single cavity. The 15 smaller oscillations between two deep minima have the periodicity of Aharonov-Bohm oscillations in the entire area covered by the 15 cavities. The observation of such faster oscillations shows that phase coherence is maintained in the experiment throughout the channel and thereby provides conclusive evidence for band structure in a lateral superlattice.

\section{b. Magnetically Induced Band Structure}

(1) Skew Minibands. The band structure in the experiment of Kouwenhoven et al. ${ }^{250}$ is present only in the quantum Hall effect regime and can thus be said to be magnetically induced. The magnetic field breaks time-reversal symmetry. Let us see what consequences that has for the band structure.

The hamiltonian in the Landau gauge $\mathbf{A}=(0, B x, 0)$ is

$$
\mathscr{H}=\frac{p_{x}^{2}}{2 m}+\frac{\left(p_{y}+e B x\right)^{2}}{2 m}+V(x, y), \quad V(x, y+a)=V(x, y),
$$

where $V$ is the periodically modulated confining potential. Bloch's theorem is not affected by the presence of the magnetic field, since $\mathscr{H}$ remains periodic in $y$ (in the Landau gauge). The eigenstates $\Psi$ have the form

$$
\Psi_{n k}(x, y)=\mathrm{e}^{i k y} f_{n k}(x, y), \quad f_{n k}(x, y+a)=f_{n k}(x, y),
$$


where the function $f$ is a solution periodic in $y$ of the eigenvalue problem

$$
\left(\frac{p_{x}^{2}}{2 m}+\frac{\left(p_{y}+\hbar k+e B x\right)^{2}}{2 m}+V(x, y)\right) f_{n k}(x, y)=E_{n}(k, B) f_{n k}(x, y) \text {. }
$$

If the wave number $k$ is restricted to the first Brillouin zone $|k|<\pi / a$, the index $n$ labels both the subbands from the lateral confinement and the minibands from the periodic modulation. Since $E$ and $V$ are real, one finds by taking the complex conjugate of Eq. (22.4) that

$$
E_{n}(k, B)=E_{n}(-k,-B) \text {. }
$$

In zero magnetic fields the energy $E$ is an even function of $k$, regardless of the symmetry of the potential $V$. This can be viewed as a consequence of timereversal symmetry. ${ }^{508}$ In nonzero magnetic fields, however, $E$ is only even in $k$ if the lateral confinement is symmetric:

$$
E_{n}(k, B)=E_{n}(-k, B) \quad \text { only if } V(x, y)=V(-x, y) .
$$

To illustrate the formation of skew minibands in a magnetically induced band structure, we consider the case of a weak periodic modulation $V_{1}(y)$ of the confining potential $V(x, y)=V_{0}(x)+V_{1}(x, y)$. The dispersion relation $E_{n}^{0}(k)$ in the absence of the periodic modulation can be approximated by

$$
E_{n}^{0}(k)=\left(n-\frac{1}{2}\right) \hbar \omega_{c}+V_{0}\left(x=-k l_{m}^{2}\right) .
$$

The index $n$ labels the Landau levels, and the wave number $k$ runs from $-\infty$ to $+\infty$. The semiclassical approximation $(22.7)$ is valid if the confining potential $V_{0}$ is smooth on the scale of the magnetic length $l_{\mathrm{m}} \equiv(\hbar / e B)^{1 / 2}$. (Equation (22.7) follows from the guiding center energy (18.1), using the identity $X \equiv-k \hbar / e B$ between the guiding center coordinate and the wave number; cf. Section 12.a.] For simplicity we restrict ourselves to the strictly one-dimensional case of one Landau level and suppress the Landau level index in what follows. To first order in the amplitude of the periodic modulation $V_{1}$, the zeroth-order dispersion relation is modified only near the points of degeneracy $K_{p}$ defined by

$$
E^{0}\left(K_{p}-p(2 \pi / a)\right)=E^{0}\left(K_{p}\right), \quad p= \pm 1, \pm 2, \ldots
$$

A gap opens near $K_{p}$, leading to the formation of a band structure as illustrated in Fig. 109. The gaps do not occur at multiples of $\pi / a$, as in a conventional 1D band structure. Moreover, the maxima and minima of two subsequent bands occur at different $k$-values. This implies indirect optical transitions between the bands if the Fermi level lies in the gap.

${ }^{508}$ L. D. Landau and E. M. Lifshitz, "Statistical Physics," Part 2, Section 55. Pergamon, Oxford, 1980. 


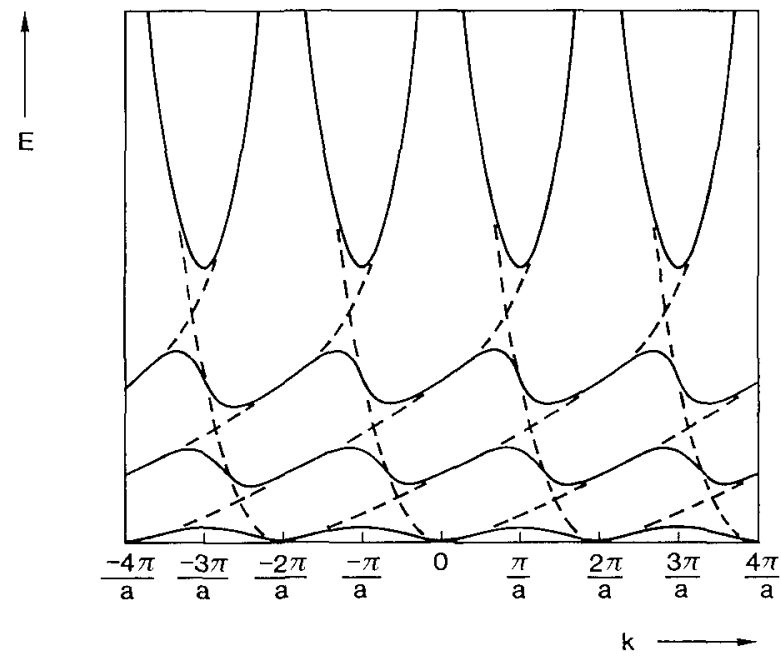

FIG 109. Illustration of magnetically induced band structure in a narrow channel with a weak periodic modulation of the confining potential $V(x)$ (for the case $V(x) \neq V(-x)$ ) The dashed curves represent the unperturbed dispersion relation (22.7) for a single Landau level. Skew minıbands result from the broken tıme-reversal symmetry in a magnetıc field

It is instructive to consider the special case of a parabolic confining potential $V_{0}(x)=\frac{1}{2} m \omega_{0}^{2} x^{2}$ in more detail, for which the zeroth-order dispersion relation can be obtained exactly (Section 10). Since the confinement is symmetric in $x$, the minigaps in this case occur at the Brillouin zone boundaries $k=p \pi / a$. Other gaps at points where the periodic modulation induces transitions between different 1D subbands are ignored for simplicity. From Eq. (10.5) one then finds that the Fermi energy lies in a minigap when

$$
E_{\mathrm{F}}=\left(n-\frac{1}{2}\right) \hbar \omega+\frac{\hbar^{2}}{2 M}\left(\frac{p \pi}{a}\right)^{2},
$$

with the definitions $\omega \equiv\left(\omega_{\mathrm{c}}^{2}+\omega_{0}^{2}\right)^{1 / 2}, M \equiv m \omega^{2} / \omega_{0}^{2}$. In the limiting case $B=0$, Eq. (22.9) reduces to the usual condition ${ }^{249}$ that Bragg reflection occurs when the longitudinal momentum $m v_{y}$ is a multiple of $\hbar \pi / a$. In the opposite limit of strong magnetic fields $\left(\omega_{c} \gg \omega_{0}\right)$, Eq. (22.9) becomes

$$
a W_{\mathrm{eff}} B=p \frac{h}{e}, \quad W_{\mathrm{eff}} \equiv 2\left(\frac{2 E_{\mathrm{G}}}{m \omega_{0}^{2}}\right)^{1 / 2} .
$$

The effective width $W_{\text {eff }}$ of the parabolic potential is the separation of the equipotentials at the guiding center energy $E_{\mathrm{G}} \equiv E_{\mathrm{F}}-\left(n-\frac{1}{2}\right) \hbar \omega_{\mathrm{c}}$.

The two-terminal conductance of the periodically modulated channel drops by $e^{2} / h$ whenever $E_{\mathrm{F}}$ lies in a minigap. If the magnetic field dependence 
of $W_{\text {eff }}$ is small, then Eq (2210) shows that the magnetoconductance oscillations have approximately the periodicity $\Delta B \sim h / e a W_{\text {eff }}$ of the Aharonov-Bohm effect in a single unit cell, in agreement with the calculations of Kouwenhoven et al $^{250}$ (Note that in their experiment the Ferm1 energy is tuned through the minigap by varying the gate voltage rather than the magnetic field ) The foregoing analysis is for a channel of infinite length The interference of reflections at the entrance and exit of a finite superlattice of length $L$ leads to transmission resonances 249387 whenever $k=p \pi / L$, as described by Eqs (229) and (22 10) after substituting $L$ for $a$ These transmission resonances are observed by Kouwenhoven et al as rapid oscillations in the conductance The number of conductance maxima between two deep minıma from the minigap equals approxımately the number $L / a$ of unit cells in the superlattice The number of maxima may become somewhat larger than $L / a$ if one takes into account reflections at the transition from a narrow channel to a wide 2DEG This might explain the observation in Ref 250 of 16 , rather than 15 , conductance maxima between two minigaps in one partıcular experıment on a 15 -perıod superlattıce

(2) Bloch Oscillations. In zero magnetic fields, an oscillatory current has been predicted to occur on application of a dc electric field to an electron gas in a periodic potential ${ }^{509}$ This Bloch oscillation would result from Bragg reflection of electrons that, accelerated by the electric field, approach the band gap A necessary condition is that the field be sufficiently weak that tunnelıng across the gap does not occur ${ }^{510} 513$ The wave number increases in time according to $\dot{k}=e E / \hbar$ in an electric field $E$ The time interval between two Bragg reflections is $2 \pi / a \dot{k}=h / e a E$ The oscillatory current thus would have a frequency $\Delta V e / h$, with $\Delta V=a E$ the electrostatic potential drop over one unit cell Bloch oscillations have so far eluded experimental observation

The successful demonstration ${ }^{250}$ of miniband formation in strong magnetic fields naturally leads to the question of whether Bloch oscillations might be observable in such a system This question would appear to us to have a negative answer The reason is simple, and it illustrates another interesting difference of magnetically induced band structure In the quantum Hall effect regime the electric field is perpendicular to the current, so no acceleration of the electrons occurs Since $\dot{k}=0$, no Bloch oscillations should be expected

${ }^{509} \mathrm{~F}$ Bloch, $Z$ Phys 52, 555 (1928)

${ }^{510} \mathrm{~J}$ N Churchill and F E Holmstrom, Phys Lett 85A, 453 (1981)

${ }^{511} \mathrm{~J}$ N Churchill and F E Holmstrom, Am J Phys 50, 848 (1982)

${ }^{512} \mathrm{~J}$ Zak, Phys Rev B 38, 6322 (1988)

${ }^{513} \mathrm{~J}$ B Krieger and G J Iafrate, Phys Rev B 38, 6324 (1988) 